\title{
British HIV Association guidelines for the treatment of HIV-1-positive adults with antiretroviral therapy 2015
}

Writing Group

Duncan Churchill,

Chair, Royal Sussex County Hospital, Brighton, UK

Laura Waters,

Vice Chair, Mortimer Market Centre, London, UK

Nadia Ahmed,

Mortimer Market Centre, London, UK

Brian Angus,

University of Oxford, UK

Marta Boffito,

Chelsea and Westminster Hospital, London, UK

Mark Bower,

Chelsea and Westminster Hospital, London, UK

David Dunn,

University College London, UK

Simon Edwards,

Central and North West London NHS Foundation Trust, UK

Carol Emerson,

Royal Victoria Hospital, Belfast, UK

Sarah Fidler,

Imperial College School of Medicine at St Mary's, London, UK

†Martin Fisher,

Royal Sussex County Hospital, Brighton, UK

Rob Horne,

University College London, UK

Saye Khoo,

University of Liverpool, UK

Clifford Leen,

Western General Hospital, Edinburgh, UK

Nicola Mackie,

Imperial College Healthcare NHS Trust, London, UK

Neal Marshall,

Royal Free Hospital NHS Trust, London, UK

Fernando Monteiro,

$U K-C A B$

Mark Nelson,

Chelsea and Westminster Hospital NHS Foundation Trust, London, UK 
Chloe Orkin,

St Bartholomew's Hospital, London, UK

Adrian Palfreeman,

Leicester Royal Infirmary, UK

Sarah Pett,

University College London, UK

Andrew Phillips,

University College London, UK

Frank Post,

King's College London, UK

Anton Pozniak,

Chelsea and Westminster Hospital NHS Foundation Trust, London, UK

lain Reeves,

Homerton University Hospital, London, UK

Caroline Sabin,

Royal Free and University College Medical School, London, UK

Roy Trevelion,

$U K-C A B$

John Walsh,

Imperial College Healthcare NHS Trust, London, UK

Ed Wilkins,

North Manchester General Hospital, UK

lan Williams,

Royal Free and University College Medical School, London, UK

Alan Winston,

Imperial College London, UK

$\dagger$ Professor Martin Fisher died in April 2015 - he made a significant contribution to these, many other guidelines and our speciality as a whole - he is greatly missed. 


\section{Table of contents}

1.0 Introduction

1.1 Scope and purpose

1.2 Methodology

1.2.1 Guideline development process

1.2.2 Involvement of PLWH.

1.2.3 GRADE

1.2.4 Good practice points

1.2.5 Dissemination and implementation

1.2.6 Guideline updates and date of next review

1.3 Treatment aims

1.4 Resource use

1.5 Implications for research

1.6 References

2.0 Summary of recommendations

3.0 Involvement of people living with HIV in decision-making...

3.1 Recommendations.

3.2 Auditable outcomes.

3.3 Rationale

3.4 References

4.0 When to start.

4.1 Chronic infection

4.1.1 Recommendations.

4.1.2 Auditable outcomes

4.1.3 Rationale

4.1.4 References

4.2 Individuals presenting with AIDS or a major infection

4.2.1 Recommendation

4.2.2 Auditable outcome

4.2.3 Rationale

4.2.4 References

4.3 Treatment of primary HIV infection.

4.3.1 Recommendation

4.3.2 Auditable outcomes

4.3.3 Rationale

4.3.4 References

4.4 Impact of treatment on prevention of onward transmission

4.4.1 Recommendations

4.4.2 Auditable outcome.

4.4.3 Rationale

4.4.4 References

5.0 What to start

5.1 Summary of recommendations

5.1.1 Summary of auditable outcomes

5.2 Introduction

5.3 Which nucleoside reverse transcriptase inhibitor backbone.

5.3.1 Recommendations

5.3.2 Rationale

5.3.3 Not recommended.

5.3.4 Lamivudine $v$ s. emtricitabine

5.3.5 References

5.4 Which third agent.

5.4.1 Recommendations

5.4.2 Rationale

5.4.3 Individual agents 


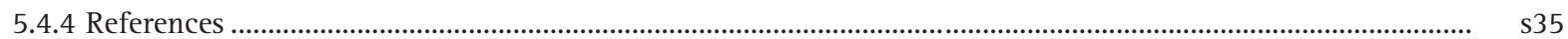

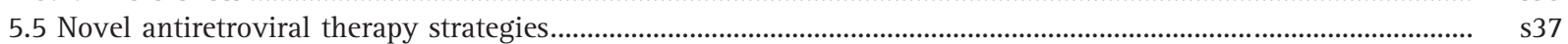

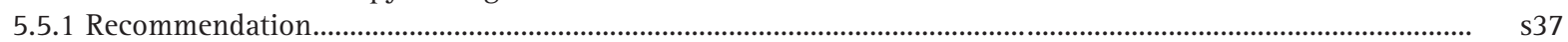

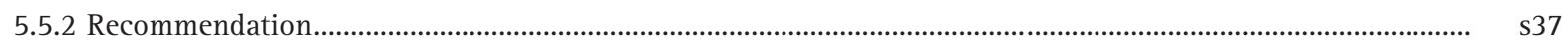

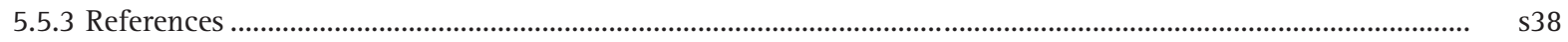

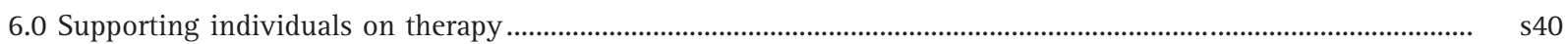

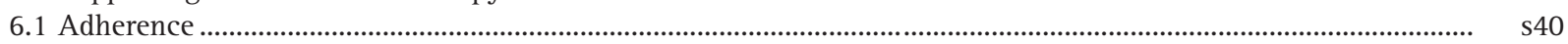

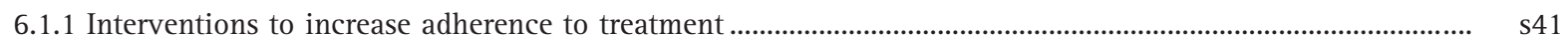

6.1.2 Should the choice of first-line antiretroviral therapy combination be affected by risk of non-adherence?.. s42

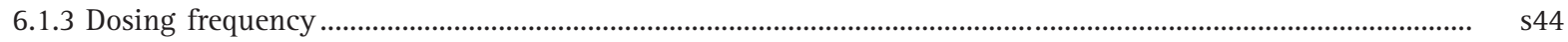

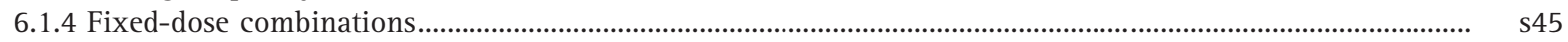

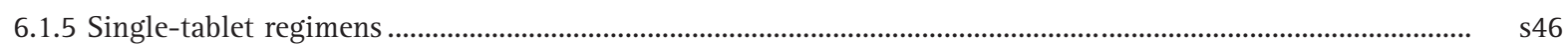

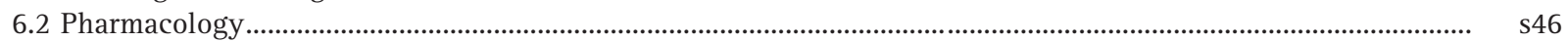

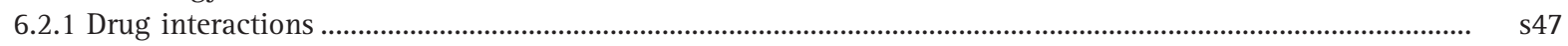

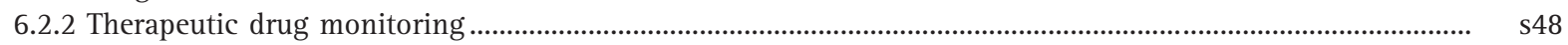

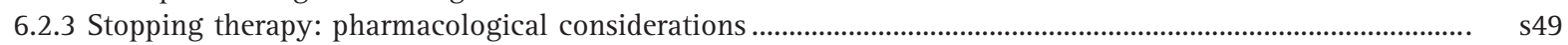

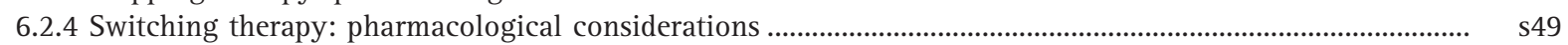

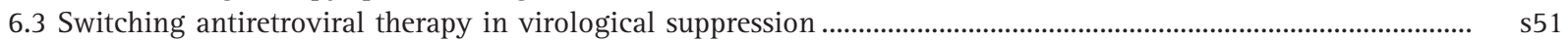

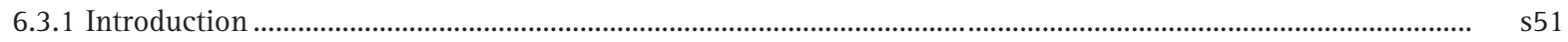

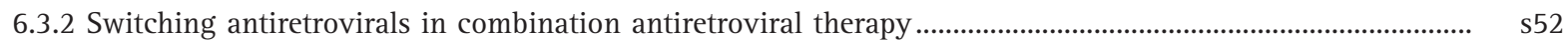

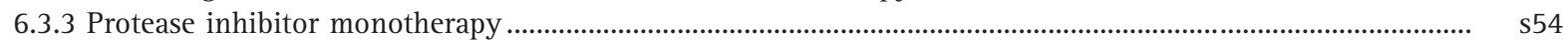

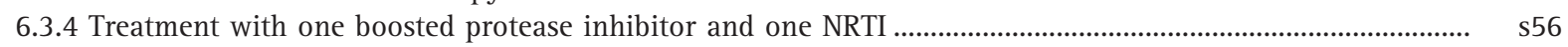

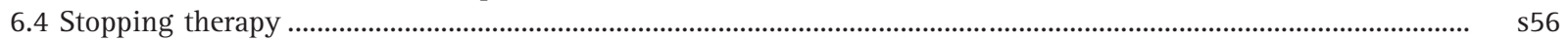

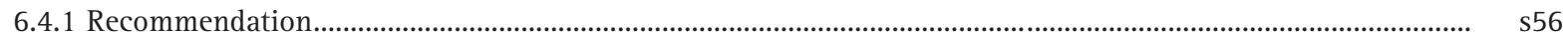

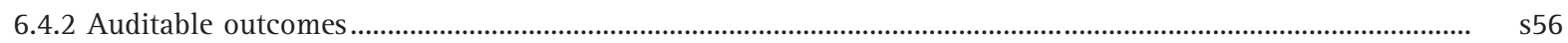

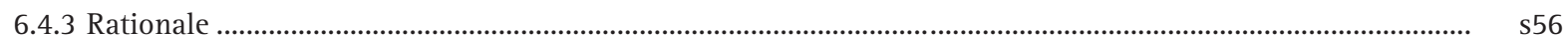

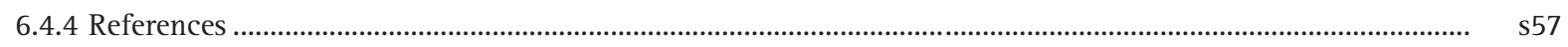

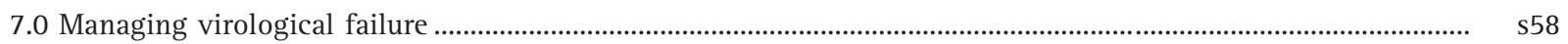

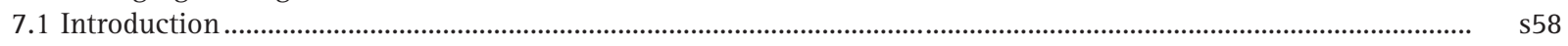

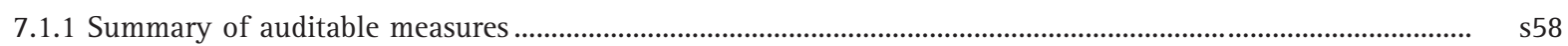

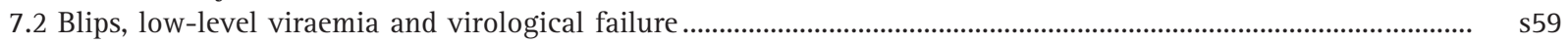

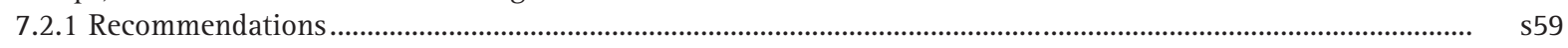

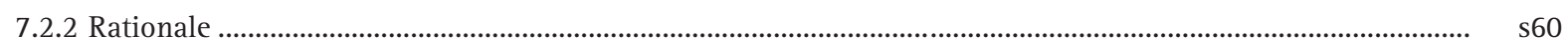

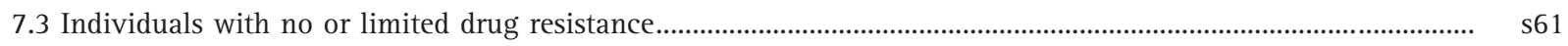

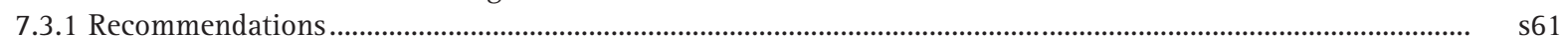

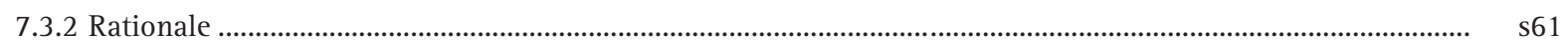

7.4 Individuals with multiple class virological failure with or without extensive drug resistance............................. s62

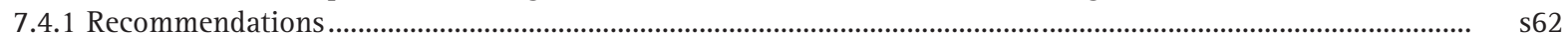

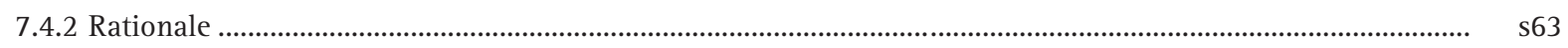

7.5 Individuals with limited or no therapeutic options when a fully viral suppressive regimen cannot be constructed s64

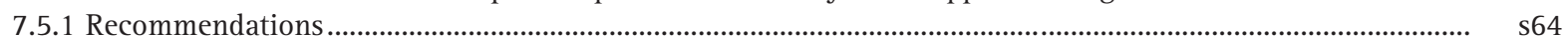

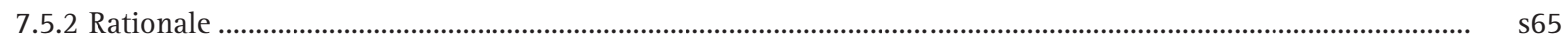

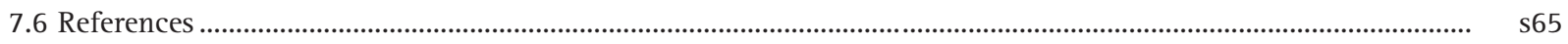

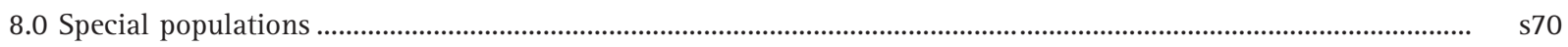

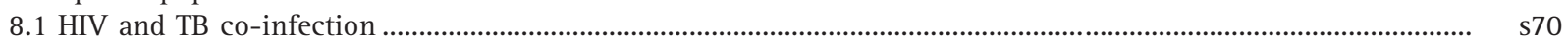

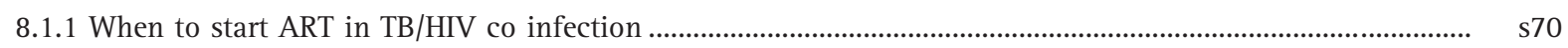

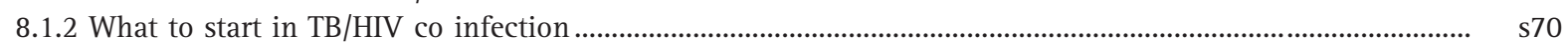

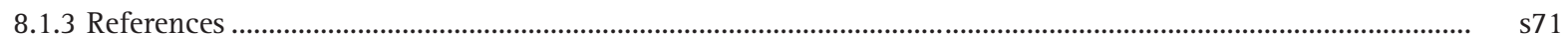

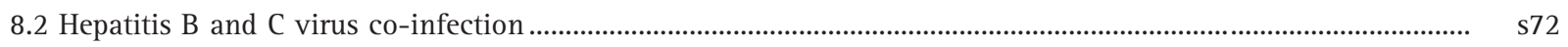

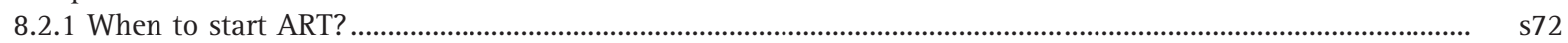

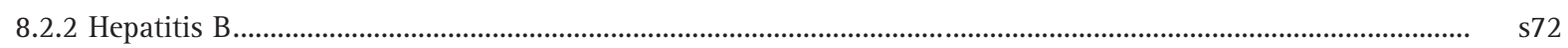

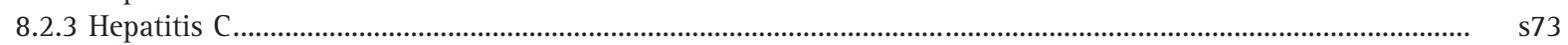

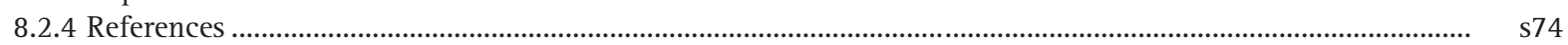

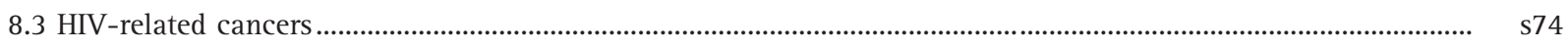

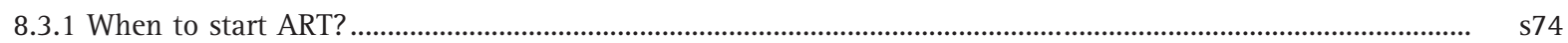


8.3.2 What to start.

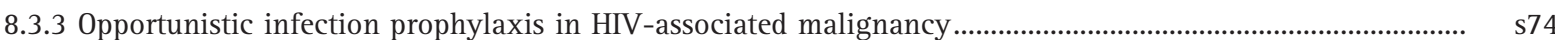

8.3.4 Other considerations from the BHIVA guidelines for HIV-associated malignancies........................................ s74

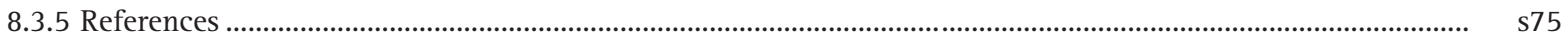

8.4 HIV-associated neurocognitive impairment ............................................................................................................. s76

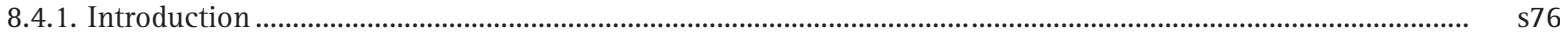

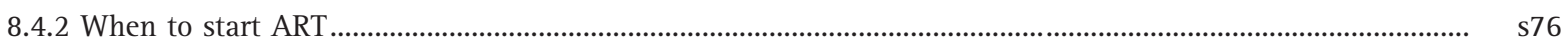

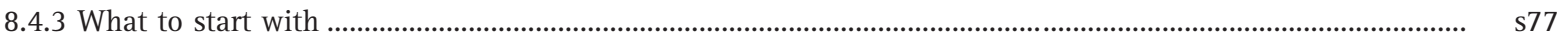

8.4.4 Continuing or worsening NC impairment despite ART …........................................................................ s78

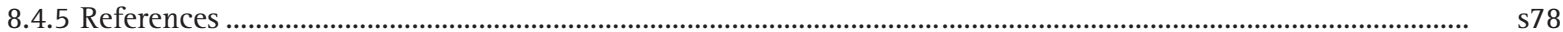

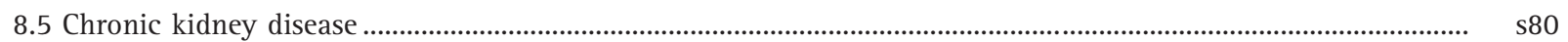

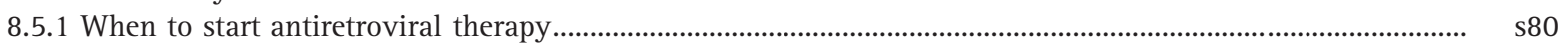

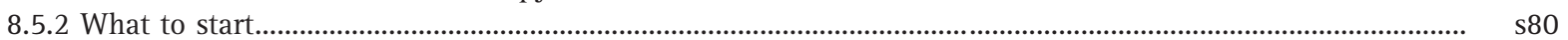

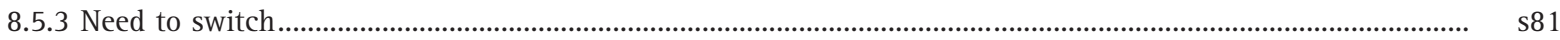

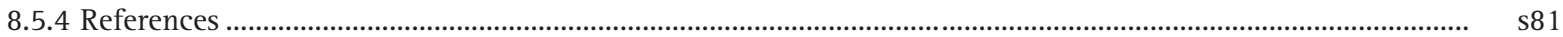

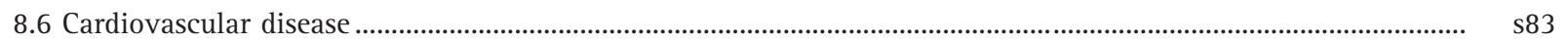

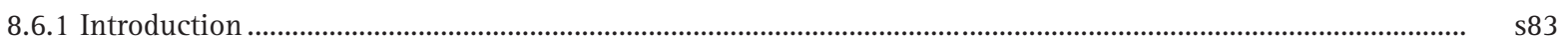

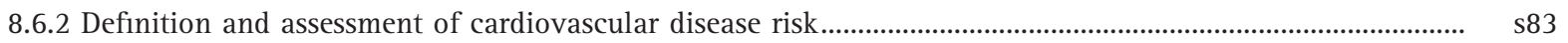

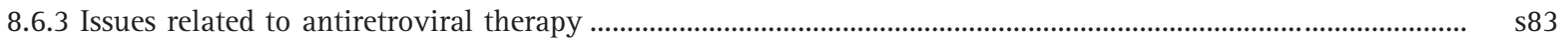

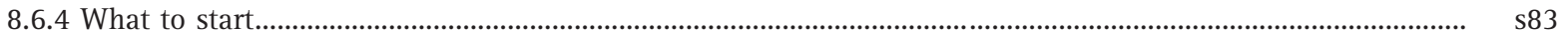

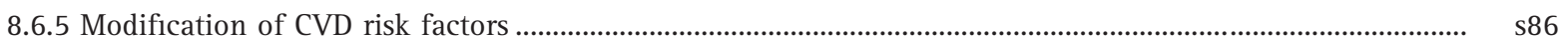

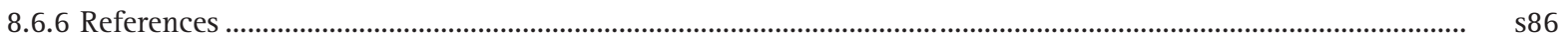

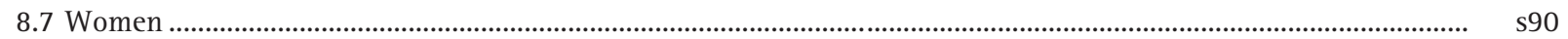

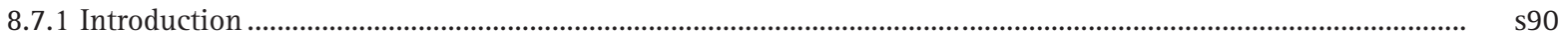

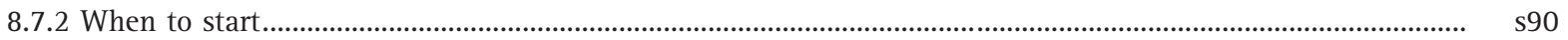

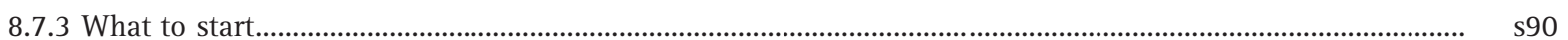

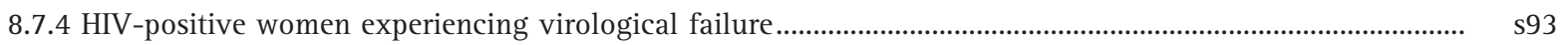

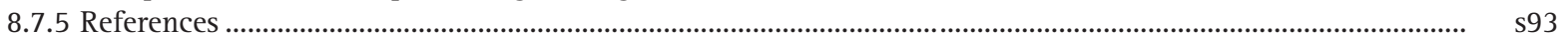

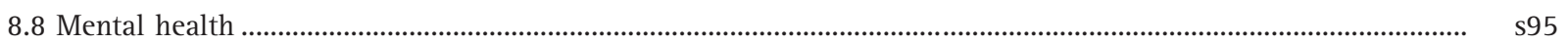

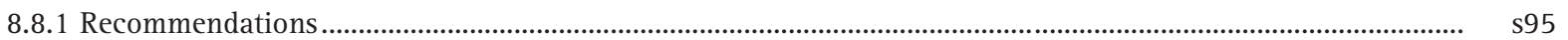

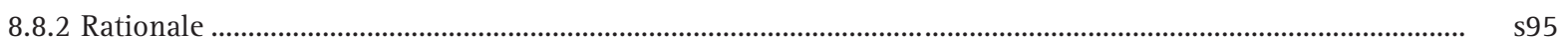

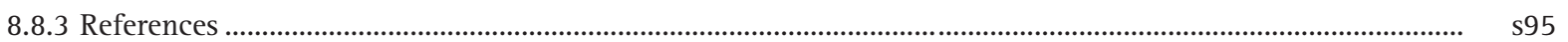

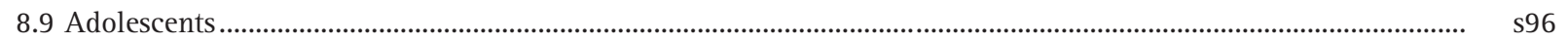

8.9.1 Recommendations for management of HIV, ART and sexual and reproductive health specifically for perinatally acquired HIV.

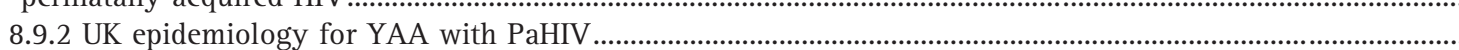

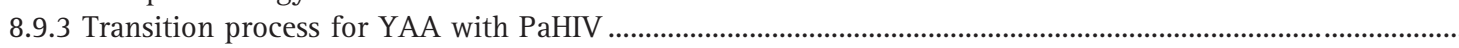

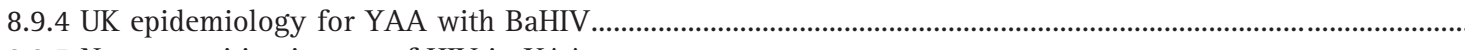

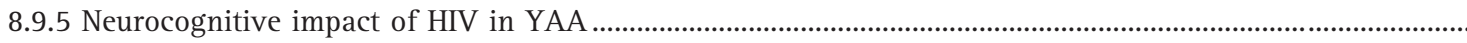

8.9.6 Antiretroviral therapy

8.9.7 References

8.10 Bone disease and antiretroviral therapy .......

8.10.1 Introduction

8.10.2 When to start antiretroviral therapy

8.10.3 What to start

8.10.4 Switching treatment...

8.11 Considerations for later life

8.11.1 Introduction

8.11.2 When to start ART

8.11.3 What to start

9.0 Acknowledgements

10.0 List of appendices 


\subsection{Introduction}

\subsection{Scope and purpose}

The overall purpose of these guidelines is to provide guidance on best clinical practice in the treatment and management of adults with HIV infection on antiretroviral therapy (ART). The scope includes: (i) guidance on the initiation of ART in those previously naïve to therapy; (ii) support of people living with HIV (PLWH) on treatment; (iii) management of individuals experiencing virological failure; and (iv) recommendations in specific populations where other factors need to be taken into consideration. The guidelines are aimed at clinical professionals directly involved with and responsible for the care of adults with HIV infection, and at community advocates responsible for promoting the best interests and care of HIV-positive adults. They should be read in conjunction with other published BHIVA guidelines.

\subsection{Methodology}

\subsubsection{Guideline development process}

BHIVA fully revised and updated the association's guideline development manual in 2011. Further updates have been carried out subsequently [1]. Full details of the guideline development process, including conflict of interest policy, are outlined in the manual. BHIVA has adopted the modified Grading of Recommendations Assessment, Development and Evaluation (GRADE) system for the assessment, evaluation and grading of evidence and development of recommendations (see below and Appendix 1) [2,3].

The scope, purpose and guideline topics were agreed by the writing group. Questions concerning each guideline topic were drafted and a systematic literature search was undertaken by an information scientist. Details of the search questions and strategy (including the definition of populations, interventions and outcomes) are outlined in Appendix 2. BHIVA guidelines for the treatment of HIV1-infected adults with antiretroviral therapy were last published in 2013 [4]. For the 2015 guidelines Medline, Embase and the Cochrane library were searched between October 2011 and August 2014. Abstracts from selected conferences (see Appendix 2) were searched between 1 January 2011 and July 2015. For each topic and healthcare question, evidence was identified and evaluated by writing group members with expertise in the field. Using the modified GRADE system, writing group members were responsible for assessing and grading the quality of evidence for predefined outcomes across studies and developing and grading the strength of recommendations. An important aspect of evaluating evidence is an understanding of the design and analysis of clinical trials, including the use of surrogate marker data. Decisions regarding the clinical importance of difference in outcomes are made by the writing group.

For a number of questions, GRADE evidence profile and summary of findings tables were constructed, using predefined and rated treatment outcomes (Appendix 3), to help achieve consensus for key recommendations and aid transparency of the process. Before final approval by the writing group, the guidelines were published online for public consultation and an external peer review was commissioned.

\subsubsection{Involvement of PLWH}

BHIVA views the involvement of PLWH and community representatives in the guideline development process as essential. The writing group included two representatives appointed through the UK HIV Community Advisory Board (UK-CAB) who were involved in all aspects of the guideline development process. Community groups were invited to participate in the draft guideline consultation process and a community consultation was held on 6th August 2015.

\subsubsection{GRADE}

The GRADE Working Group [3] has developed an approach to grading evidence that moves away from initial reliance on study design to consider the overall quality of evidence across outcomes. BHIVA has adopted the modified GRADE system for its guideline development.

The advantages of the modified GRADE system are (i) the grading system provides an informative, transparent summary for clinicians, PLWH and policy makers by combining an explicit evaluation of the strength of the recommendation with a judgement of the quality of the evidence for each recommendation, and (ii) the two-level grading system of recommendations has the merit of simplicity and provides clear direction to PLWH, clinicians and policy makers.

The strength of recommendation is graded as 1 or 2 as follows: 
- A Grade 1 recommendation is a strong recommendation to do (or not do) something, where the benefits clearly outweigh the risks (or vice versa) for most, if not all PLWH. Most clinicians and HIV-positive individuals should and would want to follow a strong recommendation unless there is a clear rationale for an alternative approach. A strong recommendation usually starts with the standard wording 'we recommend'.

- A Grade 2 recommendation is a weaker or conditional recommendation, where the risks and benefits are more closely balanced or are more uncertain. Most clinicians and PLWH would want to follow a weak or conditional recommendation but many would not. Alternative approaches or strategies may be reasonable depending on the individual HIV-positive individual's circumstances, preferences and values. A weak or conditional recommendation usually starts with the standard wording 'we suggest'.

The strength of a recommendation is determined not only by the quality of evidence for defined outcomes but also the balance between desirable and undesirable effects of a treatment or intervention, differences in values and preferences and, where appropriate, resource use. Each recommendation concerns a defined target population and is actionable.

The quality of evidence is graded from A to D and for the purpose of these guidelines is defined as the following:

- Grade A evidence means high-quality evidence that comes from consistent results from well-performed randomised controlled trials (RCTs), or overwhelming evidence of some other sort (such as well-executed observational studies with consistent strong effects and a low likelihood of uncorrected bias). Grade A implies confidence that the true effect lies close to the estimate of the effect.

- Grade B evidence means moderate-quality evidence from randomised trials that suffer from serious flaws in conduct, inconsistency, indirectness, imprecise estimates, reporting bias, or some combination of these limitations, or from other study designs with special strengths such as observational studies with consistent effects and exclusion of most potential sources of bias.

- Grade C evidence means low-quality evidence from controlled trials with several very serious limitations or observational studies with limited evidence on effects and exclusion of most potential sources of bias.

- Grade D evidence on the other hand is based only on case studies, expert judgement or observational studies with inconsistent effects and a potential for substantial bias, such that there is likely to be little confidence in the effect estimate.

\subsubsection{Good practice points}

In addition to graded recommendations, the BHIVA writing group has also included good practice points (GPP), which are recommendations based on the clinical judgement and experience of the working group. GPPs emphasise an area of important clinical practice for which there is not, nor is there likely to be, any significant research evidence. They address an aspect of treatment and care that is regarded as such sound clinical practice that healthcare professionals are unlikely to question it and where the alternative recommendation is deemed unacceptable. It must be emphasised that GPPs are not an alternative to evidence-based recommendations.

\subsubsection{Dissemination and implementation}

The following measures have or will be undertaken to disseminate and aid implementation of the guidelines:

- E-publication on the BHIVA website and the journal HIV Medicine.

- Publication in HIV Medicine.

- Shortened version detailing concise summary of recommendations.

- Shortened version for BHIVA guidelines app.

- E-learning module accredited for CME.

- Educational slide set to support local and regional educational meetings.

- National BHIVA audit programme.

\subsubsection{Guideline updates and date of next review}

The guidelines will be next fully updated and revised in 2017. However, the writing group will continue to meet regularly to consider new information from high-quality studies and publish amendments and addendums to the current recommendations before the full revision date where this is thought to be clinically important to ensure continued best clinical practice.

\subsection{Treatment aims}

The primary aim of ART is the prevention of the mortality and morbidity associated with chronic HIV infection at low cost of drug toxicity. Treatment should improve the physical and psychological well-being of PLWH. The effectiveness and tolerability of ART has improved significantly over the last 15 years. The overwhelming majority of PLWH attending HIV services in the UK and receiving ART experience long-term virological suppression and good treatment outcomes [5], which compare very 
favourably with other developed countries. Notably, in 2013 around 90\% of those diagnosed with HIV in the UK had initiated ART, with 93\% of those on ART having a suppressed viral load [5].

A UK analysis of individuals commencing ART between 2000 and 2010 demonstrates that the expected age at death for HIV-positive men from ART start was 68, 73 and 77 years at exact ages 20, 35 and 50 years, respectively, compared with 77,78 and 79 years in the general population [6]. The corresponding expected age at death for HIV-positive women was 69, 74 and 78 years, compared with 81,82 and 83 years in the general population. The same study shows that life expectancy in men and women with an undetectable viral load and CD4 cell count greater than 350 cells $/ \mu \mathrm{L}$ is the same as, or slightly better than, that for the general population [6]. Modelling has suggested that for HIV-positive men who have sex with men (MSM) living in a developed country with extensive access to HIV care and assuming a high rate of HIV diagnosis, the life expectancy is 75 years [7]. The authors concluded that the greatest risk of excess mortality is due to delays in HIV diagnosis. Decreasing late diagnosis (and consequently starting ART earlier), maintaining individuals in care and reducing long-term drug toxicity and non-aquired immune deficiency syndrome (AIDS) co-morbidities are crucial to further improving life expectancy and the well-being of people living with HIV infection.

A further aim of treatment is the reduction in sexual transmission of HIV. The use of ART to prevent motherto-child transmission is universally accepted and best practice is addressed in the BHIVA guidelines for the management of HIV infection in pregnant women [8]. Recently, the size of the effect of ART on reducing the risk of sexual transmission of HIV has been estimated at $>95 \%[9,10]$. The PARTNER study investigated the risk of HIV transmission within serodifferent couples where the HIV-positive partner was on suppressive ART (viral load less than 200 copies $/ \mathrm{mL}$ ) and demonstrated no transmissions compared with a predicted 86 new infections had the positive partner not been on effective ART [11]. The upper 95\% confidence interval for risk of transmission was 0.4 per 100 couple years for any sex and 1.0 per 100 couple years for anal sex. At a population level, ART is likely to be important in reducing the incidence of HIV infection.

\subsection{Resource use}

ART is extremely cost-effective and compares favourably with the cost of management of many other chronic diseases. Estimates of the cost-effectiveness of ART have been assessed in studies in North America and Europe [12-14]. Their findings have been consistent with an estimated incremental cost-effectiveness ratio of about US $\$ 20,000$ per quality adjusted life year (QALY) for combination ART compared with no therapy based on drug costs and treatment patterns in the USA and Europe [15]. The primary aim of these guidelines is to summarise and base recommendations on the clinical benefits of ART and different ART options.

The number of people living with HIV in the UK continues to increase and by the end of 2013 was estimated to be 107,800 (95\% credible interval 101,600-115,800), of whom 24\% were undiagnosed. In 2013, 90\% (73,290/ $81,510)$ of people seen for HIV care were prescribed ART [5]. With ongoing HIV transmission, increased HIV testing and a reduction in the undiagnosed fraction, the number of people diagnosed with HIV and accessing HIV services will continue to increase. It has been estimated that the annual population treatment and care costs rose from $£ 104$ million in 1997 to $£ 483$ million in 2006 , rising to a projected annual cost of $£ 721$ million in 2013 [16]. It is likely this estimated projected cost is an overestimate due to various factors, including earlier diagnosis and a lower proportion of individuals with symptoms. However, in the current economic climate containing and reducing costs without affecting the current high standards of care and treatment outcomes will be an immense challenge to commissioners, healthcare professionals and PLWH alike. A collaborative approach is required.

In the UK, higher annual treatment and care costs have been associated with late diagnosis and initiation of ART at lower CD4 cell counts than the BHIVA guidelines recommend $[17,18]$. In addition to earlier diagnosis and initiation of ART, reducing inpatient episodes, decreasing drug toxicity, preventing HIV-associated co-morbidities and innovations in models of care are likely to have a beneficial effect on costs. However, the cost of antiretroviral (ARV) drugs remains the major factor contributing to treatment and care costs. With the increasing availability of generic drugs and the introduction of a standard tariff for HIV services (in England), commissioners and the NHS will be faced with difficult choices about the value and benefit of different ARV drugs.

The BHIVA writing group recognises that cost of drugs is an important issue in the choice of ART regimens. In addition to drug acquisition costs there are costs associated with, for example, multidisciplinary team meetings, switching ART, co-morbidities and management of drugdrug interactions. There are limited cost-effectiveness data in the UK comparing different ARV drugs and for this reason we did not include cost-effectiveness as an 
outcome in ART comparisons. However, the writing group believes that decreasing the risks of virological failure, drug resistance and drug-associated toxicity are likely to have a beneficial impact on long-term costeffectiveness and resource use. In the setting of similar virological efficacy, determining the acceptable threshold at which differences in the risk of toxicity, tolerability and convenience outweigh differences in resource use and cost will be important. These thresholds may differ among clinicians and PLWH alike.

In developing the recommendations in these guidelines, we have taken into account differences in critical treatment outcomes between different drug regimens in determining preferred and alternative treatment regimens. We recognise and support that commissioning arrangements and local drug costs will and should influence ART choice where outcomes, across a range of clinical measures, are similar between individual drugs in the treatment of defined populations. However, we believe that reducing treatment costs should not be at the cost of an increased risk of poorer treatment outcomes and quality of care, not least as these are likely to have a detrimental impact on long-term cost.

\subsection{Implications for research}

In reviewing quality of evidence, guidelines will identify areas of treatment and care where there is an absence of evidence or limited confidence in the size of effect to influence choice of treatments or determine treatment and management strategies. For this reason, it is not the intention of these guidelines to stifle clinical research but rather to help promote continued research with the aim to further improve clinical care and treatment outcomes. The development and provision of HIV clinical trials within the UK are supported, and participation in a clinical trial should be open and offered to PLWH where appropriate.

\subsection{References}

1 BHIVA. BHIVA Guideline Development Manual. 28 January 2014. Available at: www.bhiva.org/

GuidelineDevelopmentManual.aspx (accessed August 2015).

2 Guyatt GH, Oxman AD, Kunz R et al. Going from evidence to recommendations. BMJ 2008; 336: 1049-1051.

3 GRADE Working Group. Grading the quality of evidence and the strength of recommendations. Available at: www.gradeworkinggroup.org/intro.htm (accessed August 2015).

4 Williams I, Churchill D, Anderson J et al. British HIV Association guidelines for the treatment of HIV-1-positive adults with antiretroviral therapy 2012 (Updated November 2013. All changed text is cast in yellow highlight.). HIV Med 2014; 15 (Suppl 1): 1-85.

5 Yin Z, Brown AE, Hughes G et al. HIV in the United Kingdom 2014 Report: data to end 2013. London: Public Health England; November 2014. Available at: https:// www.gov.uk/government/statistics/hiv-in-the-unitedkingdom (accessed August 2015).

6 May MT, Gompels M, Delpech V et al. Impact on life expectancy of HIV-1 positive individuals of CD4 + cell count and viral load response to antiretroviral therapy. AIDS 2014; 28: 1193-1202.

7 Nakagawa F, Lodwick RK, Smith CJ et al. Projected life expectancy of people with HIV according to timing of diagnosis. AIDS 2012; 26: 335-343.

8 de Ruiter A, Taylor GP, Clayden P et al. British HIV Association guidelines for the management of HIV infection in pregnant women 2012 (2014 interim review). HIV Med 2014; 15 (Suppl 4): 1-77.

9 Cohen MS, Chen YQ, McCauley M et al. Prevention of HIV1 infection with early antiretroviral therapy. $N$ Engl J Med 2011; 365: 493-505.

10 Attia S, Egger M, Muller M et al. Sexual transmission of HIV according to viral load and antiretroviral therapy: systematic review and meta-analysis. AIDS 2009; 23: 1397-1404.

11 Rodger A, Bruun T, Cambiano V et al. HIV transmission risk through condomless sex if HIV+ partner on suppressive ART: PARTNER study. Conference on Retroviruses and Opportunistic Infections. March 2014. Boston, MA, USA [Abstract 153LB].

12 Sendi PP, Bucher HC, Harr T et al. Cost effectiveness of highly active antiretroviral therapy in HIV-infected patients. Swiss HIV Cohort Study. AIDS 1999; 13: 1115-1122.

13 Miners AH, Sabin CA, Trueman P et al. Assessing the costeffectiveness of HAART for adults with HIV in England. HIV Med 2001; 2: 52-58.

14 Freedberg KA, Losina E, Weinstein MC et al. The cost effectiveness of combination antiretroviral therapy for HIV disease. N Engl J Med 2001; 344: 824-831.

15 Yazdanpanah Y. Costs associated with combination antiretroviral therapy in HIV-infected patients. J Antimicrob Chemother 2004; 53: 558-561.

16 Mandalia S, Mandalia R, Lo G et al. Rising population cost for treating people living with HIV in the UK, 1997-2013. PLoS One 2010; 5: e15677.

17 Beck EJ, Mandalia S, Sangha R et al. The cost-effectiveness of early access to HIV services and starting cART in the UK 1996-2008. PLoS One 2011; 6: e27830.

18 Beck EJ, Mandalia S, Lo G et al. Cost-effectiveness of early treatment with first-line NNRTI-based HAART regimens in the UK, 1996-2006. PLoS One 2011; 6: e20200. 


\subsection{Summary of recommendations}

3.0 Involvement of people living with HIV in decision-making

3.1 Recommendations

- We recommend PLWH are given the opportunity to be involved in making decisions about their treatment (GPP).

- Provision of treatment-support resources should include in-house, independent and community information providers and peer-support resources (GPP).

4.0 When to start

4.1 Chronic infection

4.1.1 Recommendations

- We recommend people with HIV start ART (1A).

4.2 Individuals presenting with AIDS or a major infection

4.2.1 Recommendation

- We recommend that individuals presenting with an AIDS-defining infection, or with a serious bacterial infection and a CD4 cell count $<200$ cells $/ \mu \mathrm{L}$, start ART within 2 weeks of initiation of specific antimicrobial chemotherapy (1B).

4.3 Treatment of primary HIV infection

4.3.1 Recommendation

- We recommend all individuals with suspected or diagnosed PHI are reviewed promptly by an HIV specialist and offered immediate ART (1B).

4.4 Impact of treatment on prevention of onward transmission 4.4.1 Recommendations

- We recommend that ART is offered to all PLWH for the prevention of onward transmission (1A).

- We recommend the evidence that treatment with ART substantially lowers the risk of transmission is discussed with all PLWH (GPP).

- An assessment of the risk of transmission to others should be made at diagnosis and subsequent visits (GPP).

5.0 What to start

5.1 Summary recommendations

- We recommend that therapy-naïve PLWH start ART containing two nucleoside reverse transcriptase inhibitors (NRTIs) plus one of the following: ritonavir-boosted protease inhibitor $(\mathrm{Pl} / \mathrm{r})$, non-nucleoside reverse transcriptase inhibitor (NNRTI) or integrase inhibitor (INI) (1A).

Table 5.1.1 Summary recommendations for choice of ART

\begin{tabular}{cll}
\hline & Preferred & Alternative \\
\hline NRTI backbone & $\begin{array}{c}\text { Tenofovir-DF and } \\
\text { emtricitabine } \\
\text { Third agent (alphabetical } \\
\text { order) }\end{array}$ & $\begin{array}{c}\text { Abacavir and } \\
\text { lamivudine }\end{array}$ \\
& $\begin{array}{l}\text { Darunavir/r } / r \\
\text { Efavirenz }\end{array}$ \\
& Dolutegravir & \\
& Elvitegravir/c & \\
& Raltegravir $^{\mathrm{a}}$ & \\
& Rilpivirine $^{\mathrm{d}}$ & \\
&
\end{tabular}

/r: boosted with ritonavir; /c: boosted with cobicistat.

abacavir is contraindicated if an individual is HLA-B*57:01 positive.
${ }^{b}$ Use recommended only if baseline viral load is $<100,000$ copies/ $\mathrm{mL}$ except when initiated in combination with dolutegravir in which case abacavir/lamivudine can be used at any baseline viral load.

'Tenofovir-DF/emtricitabine/elvitegravir/c fixed-dose combination should not be initiated in individuals with creatinine clearance $<70 \mathrm{~mL} / \mathrm{min}$.

${ }^{\mathrm{d}}$ Use recommended only if baseline viral load is $<100,000$ copies $/ \mathrm{mL}$.

NB. The viral load advice for abacavir/lamivudine and rilpivirine applies only to initiating these agents in individuals with a detectable viral load - when these agents are used as a switch option in the context of viral load suppression the baseline viral load can be disregarded.

5.3 Which nucleoside reverse transcriptase inhibitor backbone

5.3.1 Recommendations

- We recommend therapy-naïve individuals start combination ART containing tenofovir-DF and emtricitabine as the preferred NRTI backbone (1A).

- We suggest abacavir and lamivudine is an acceptable alternative NRTI backbone in therapy-naïve individuals. In those with a baseline viral load $>100,000$ copies $/ \mathrm{mL}$, it should be used with caution if there are clinical reasons to prefer over tenofovir-DF and emtricitabine (2A).

- The caution regarding baseline viral load does not apply if abacavir/ lamivudine is used with dolutegravir (2A).

- Abacavir must not be used in individuals who are HLA-B*57:01-positive (1A).

5.4 Which third agent

5.4.1 Recommendations

- We recommend therapy-naïve individuals start combination ART containing atazanavir/r, darunavir/r, dolutegravir, elvitegravir/c, raltegravir or rilpivirine as the third agent $(1 \mathrm{~A})$.

- We suggest that for therapy-naïve individuals, efavirenz is an acceptable alternative third agent $(1 \mathrm{~A})$.

5.5 Novel antiretroviral therapy strategies

5.5.1 Recommendation

- We recommend against the use of PI monotherapy as initial therapy for treatment-naïve patients (1C).

5.5.2 Recommendation

- We suggest the use of darunavir/r-based dual ART regimen with raltegravir in treatment-naïve patients with CD4 count $>200$ cells $/ \mu \mathrm{L}$ and viral load $<100,000$ copies $/ \mathrm{mL}$ where there is need to avoid abacavir or and tenofovir-DF (2A).

- We recommend against the use of PI-based dual ART with a single NNRTI, NRTI or CCR5 receptor antagonist for treatment-naïve patients (1B).

6.0 Supporting individuals on therapy

6.1 Adherence

6.1.1 Interventions to increase adherence to treatment

6.1.1.1 Recommendations

- We recommend adherence and potential barriers to it are assessed and discussed with PLWH whenever ART is discussed, prescribed or dispensed (GPP).

- We recommend adherence support should address both perceptual barriers (e.g. beliefs and preferences) and/or practical barriers (e.g. limitations in capacity and resources) to adherence (GPP).

- Individuals experiencing difficulties with adherence should be offered additional support from staff within the MDT who have experience in adherence support and/or from organisations offering peer support (GPP). 
6.1.2 Should the choice of first-line antiretroviral therapy combination be affected by risk of non-adherence?

6.1.2.1 Recommendation

- In individuals where there is clinical concern that doses may be missed intermittently, there is insufficient evidence to recommend a $\mathrm{Pl} / \mathrm{r}$ over NNRTI- or INI-based regimens. However, where there is a risk of frequent prolonged treatment interruptions, $\mathrm{Pl} / \mathrm{r}$-based regimens may be associated with less frequent selection for drug resistance (2C).

6.2 Pharmacology

6.2.1 Drug interactions

6.2.1.1 Recommendations

- Drug histories should be taken at each clinic visit, and a full medication history (including herbals, recreational drugs and other non-prescribed medications) should be undertaken at least annually (GPP).

- All potential adverse pharmacokinetic interactions between antiretroviral drugs and other concomitant medications should be checked before administration (with tools such as www.hiv-druginteractions.org) (GPP).

- Wherever feasible, PLWH should be counselled about the risks of drug interactions, and advised to use resources such as the Liverpool HIV Drug Interactions App (iOS or Android) (GPP).

6.2.2 Therapeutic drug monitoring

6.2.2.1 Recommendation

- We recommend against the unselected use of therapeutic drug monitoring (TDM) (GPP)

- TDM may be of clinical value in specific populations (e.g. children, pregnant women) or selected clinical scenarios (e.g. malabsorption, drug interactions, suspected non-adherence to therapy) (2C).

6.2.3 Stopping therapy: pharmacological considerations

6.2.3.1 Recommendations

- We recommend individuals stopping ART containing an NNRTI in combination with an NRTI backbone replace all drugs with a PI (darunavir/ $r$ once daily) for 4 weeks (1C).

- We recommend individuals stopping a PI-containing regimen stop all drugs simultaneously and no replacement is required (1C).

6.3.2 Switching antiretrovirals in combination antiretroviral therapy 6.3.2.1 Recommendations

- We recommend, in individuals on suppressive ART regimens, consideration is given to differences in side-effect profile, drug-drug interactions and drug-resistance patterns before switching any ARV component (GPP).

- In individuals with previous NRTI resistance mutations, we recommend against switching a $\mathrm{PI} / \mathrm{r}$ to either an NNRTI or an INI as the third agent (1B).

6.3.3 Protease inhibitor monotherapy

6.3.3.1 Recommendation

- We recommend against the use of PI monotherapy for routine ART $(1 \mathrm{~A})$.

- We recommend against the use of PI monotherapy for individuals whose initial regimen has failed or who have established resistance to one more antiretroviral drugs $(1 \mathrm{~A})$.

6.3.4 Treatment with one boosted protease inhibitor and one NRTI

6.3.4.1 Recommendation

- We suggest that a boosted PI plus lamivudine as an alternative to three-drug ART in individuals with viral suppression (2A).

6.4 Stopping therapy

6.4.1 Recommendation

- We recommend against treatment interruption or intermittent therapy in individuals stable on a virally suppressive ART regimen (1A).

7.0 Managing virological failure

7.2 Blips, low-level viraemia and virological failure

7.2.1 Recommendations

In individuals on ART:
- A single VL 50-200 copies/mL preceded and followed by an undetectable VL is usually not a cause for clinical concern (GPP).

- We recommend that a single $\mathrm{VL}>200$ copies $/ \mathrm{mL}$ is investigated further, including a genotypic resistance test, as it is indicative of virological failure (1C).

- We recommend that in the context of repeated viral blips, resistance testing be attempted (1D).

7.3 Individuals with no or limited drug resistance

7.3.1 Recommendations

- We recommend for individuals experiencing virological failure on firstline ART with WT virus at baseline and without emergent resistance mutations at failure, switch to a $\mathrm{Pl} / \mathrm{r}$-based combination ART regimen is the preferred option (1C).

- We recommend individuals experiencing virological failure on first-line ART with WT virus at baseline and limited emergent resistance mutations (including two-class NRTI/NNRTI) at failure, switch to a new $\mathrm{PI} / \mathrm{r}$ based regimen with the addition of at least one, preferably two, active drugs (1C).

- We recommend individuals experiencing virological failure on first-line $\mathrm{Pl} / \mathrm{r}$ plus two-NRTI-based regimens, with limited major protease mutations, switch to a new active $\mathrm{Pl} / \mathrm{r}$ with the addition of at least one, preferably two, active agents of which one has a novel mechanism of action $(1 \mathrm{C})$.

- We recommend against switching a PI/r to an INI or NNRTI as the third agent in individuals with historical or existing reverse transcriptase mutations associated with NRTI resistance or past virological failure on NRTIs (1B).

7.4 Individuals with multiple class virological failure with or without extensive drug resistance

7.4.1 Recommendations

- We recommend individuals with persistent viraemia and with limited options to construct a fully suppressive regimen are discussed/referred for expert advice (or through virtual clinic referral) (GPP).

- We recommend individuals with extensive drug resistance are switched to a new ART regimen containing at least two and preferably three fully active agents with at least one active $\mathrm{PI} / \mathrm{r}$ such as darunavir/r and one agent with a novel mechanism (an INI, maraviroc or enfuvirtide) with etravirine an option based on viral susceptibility (1C).

- We recommend individuals with extensive drug resistance including reduced darunavir susceptibility receive dolutegravir as the INI (1C).

- We suggest that consideration on an individual basis should be given to whether inclusion of NRTls with reduced activity on genotypic testing will provide additional antiviral activity if the regimen includes three fully active drugs including a boosted $\mathrm{PI}(2 \mathrm{C})$.

- We recommend all individuals receive intensive adherence support at the start and at regular intervals to support them on their new ART combination (GPP).

7.5 Individuals with limited or no therapeutic options when a fully viral suppressive regimen cannot be constructed

7.5.1 Recommendations

- We recommend accessing newer agents through research trials, expanded access and named individual programmes (GPP).

- We suggest that consideration on an individual basis should be given to whether inclusion of NRTls with reduced activity on genotypic testing will provide additional antiviral activity - this may well be the case where it is difficult to construct a regimen with three fully active drugs including a boosted PI (see previous Section) (2C).

- We recommend against discontinuing or interrupting ART (1B).

- We recommend against adding a single, fully active ARV because of the risk of further resistance (1D).

- We recommend against the use of maraviroc to increase the CD4 cell count in the absence of CCR5 tropic virus (1C)

- We recommend that in the context of triple class failure and raltegravir/elvitegravir selected integrase resistance, twice daily dolutegravir should be included as part of a new regimen where there is at least one fully active agent in the background regimen (1C). 
8.0 Special populations

8.1 HIV and TB co-infection

8.1.1 When to start ART in TB/HIV co infection

8.1.1.1 Recommendations

- We recommend all patients with HIV TB co-infection start ART (1B)

- We recommend individuals with CD4 cell count $<50$ cells $/ \mu \mathrm{L}$ start ART as soon as TB treatment is tolerated and wherever possible within 2 weeks $(1 \mathrm{~B})$

- We recommend that for individuals with CD4 cell count $\geq 50$ cells $/ \mu \mathrm{L}$, ART can be deferred until between 8 and 12 weeks of TB treatment especially when there are difficulties with drug-drug interactions, adherence and toxicities (1B). (Although the data suggest a cut-off of 50 cells/ $\mu \mathrm{L}$, because of the daily variability in CD4, a cut-off of 100 cells/ $\mu \mathrm{L}$ may be more appropriate.)

8.1.2 What to start in TB/HIV co infection

8.1.2.1 Recommendations

- We recommend efavirenz in combination with tenofovir-DF and emtricitabine as first-line ART (1B) in TB/HIV coinfection

- We recommend that when rifampicin is used with efavirenz standard doses of efavirenz are given whatever the body weight (1B)

- We suggest that raltegravir should be used with caution with rifampi$\operatorname{cin}(2 \mathrm{C})$

- We suggest dolutegravir is a possible alternative agent (for which there is currently little evidence) but the dose should be increased to $50 \mathrm{mg}$ bd (2D)

- We recommend frequent viral load monitoring if INIs are used (1C)

- We recommend that rifampicin is not used with either NVP or a regimen containing ritonavir or cobicistat (1C)

- We recommend that where effective ART necessitates the use of ritonavir or cobicistat, that rifabutin is used instead of rifampicin (1C)

8.2 Hepatitis $B$ and $C$ virus co-infection

8.2.1 When to start ART in HBV co-infection

Table 8.2.1 Summary recommendations for the treatment of hepatitis B and C co-infection

\begin{tabular}{|c|c|c|c|}
\hline $\begin{array}{l}\text { HBV requiring } \\
\text { treatment* }\end{array}$ & $\begin{array}{l}\text { HBV not } \\
\text { requiring } \\
\text { treatment }\end{array}$ & $\begin{array}{l}\text { HCV with immediate } \\
\text { plan to start HCV } \\
\text { treatment* }\end{array}$ & $\begin{array}{l}\text { HCV with no } \\
\text { immediate } \\
\text { plan to } \\
\text { start HCV } \\
\text { treatment }\end{array}$ \\
\hline $\begin{array}{l}\text { Start ART } \\
\text { promptly (1A) } \\
\text { (include } \\
\text { tenofovir-DF } \\
\text { and } \\
\text { emtricitabine) }\end{array}$ & $\begin{array}{l}\text { Start ART (1A) } \\
\quad \text { (include } \\
\text { tenofovir-DF } \\
\text { and } \\
\text { emtricitabine) }\end{array}$ & $\begin{array}{l}\text { Start ART before } \\
\text { HCV treatment } \\
\text { commenced (1C); } \\
\text { acceptable to } \\
\text { defer if CD4 cell } \\
\text { count }>500 \text { cells/ } \\
\mu \mathrm{L} \text {. Discuss with } \\
\text { HIV and viral } \\
\text { hepatitis specialist }\end{array}$ & Start ART (1A) \\
\hline
\end{tabular}

*See BHIVA guidelines for the management of hepatitis viruses in adults (www.bhiva.org/hepatitis-guidelines.aspx) for indications to treat hepatitis B and C.
8.2.2 Hepatitis B

8.2.2.1 When to start antiretroviral therapy in HBV co-infection

8.2.2.1.1 Recommendations

- We recommend individuals with HIV and hepatitis B virus (HBV) coinfection are treated with fully suppressive ART inclusive of anti-HBV active antivirals, regardless of CD4 cell count (1A).

- We recommend individuals with HIV and HBV co-infection who have an HBV-DNA $\geq 2000 \mathrm{IU} / \mathrm{mL}$ and/or evidence of more than minimal fibrosis (Metavir $\geq F 2$ ) are treated with fully suppressive ART inclusive of anti-HBV active antivirals promptly $(1 \mathrm{C})$

8.2.2.2 What to start in HBV co-infection 8.2.2.2.1 What to start recommendations

- We recommend tenofovir-DF/emtricitabine as part of a fully suppressive ART combination should be given to all individuals starting HIV treatment (1C).

- We recommend neither lamivudine nor emtricitabine be used as the sole active drug against HBV in ART due to the rapid emergence of HBV resistant to these agents (1B).

- We recommend lamivudine/emtricitabine may be omitted from the ART regimen and tenofovir-DF be given as the sole anti-HBV active agent if there is clinical or genotypic evidence of lamivudine/emtricitabine-resistant HBV or HIV (1D).

8.2.3 Hepatitis C

8.2.3.1 When to start antiretroviral therapy in HCV co-infection 8.2.3.1.1 Recommendations

- We recommend all individuals with HIV and hepatitis C virus (HCV) coinfection be assessed for HCV treatment (GPP).

- We recommend commencing ART regardless of CD4 cell count (1A).

- We recommend HCV be considered an additional factor supporting ART in individuals with CD4 $>500$ cells/ $\mu \mathrm{L}$ who are uncertain about commencing ART (2C)

- We suggest treating HCV before commencing ART is an option if there are concerns about drug-drug interactions or adherence (GPP)

8.3.2.2 What to start in HCV co-infection

8.3.2.2.1 Recommendations

- We recommend if individuals are commencing ART, and direct-acting antivirals are not being considered, standard first-line ART should be commenced (GPP).

- We recommend that when direct-acting antivirals are to be used, there is careful consideration of possible drug-drug interactions (1C) and current or archived HIV resistance. All drug interactions should be checked with an expert source (e.g. www.hiv-druginteractions.org).

- We suggest that if abacavir is to be used with ribavirin, the ribavirin should be weight-based dose-adjusted (2C)

8.3 HIV-related cancers

8.3.1 When to start ART

8.3.1.1 AIDS-defining malignancies

- We recommend that all patients with AIDS-defining malignancies should start ART promptly (1B).

8.3.1.1.1 Kaposi sarcoma (KS)

- We recommend that ART should be started promptly in all individuals diagnosed with KS (1B).

8.3.1.1.2 Non-Hodgkin lymphoma (NHL)

- We recommend that chemotherapy regimens should be combined with ART therapy in Burkitt lymphoma and diffuse large B-cell lymphoma (1B).

- We recommend that all individuals with primary effusion lymphoma (PEL), plasmablastic lymphoma and primary central nervous system lymphoma should be started on ART if not already on it (1C). 
8.3.1.1.3 Cervical cancer

- We suggest that women with $\mathrm{CIN} 2 / 3$ should commence ART promptly (2B).

- We recommend that all women living with HIV who are to be treated with chemo-radiotherapy (CRT) for cervical cancer CRT should start ART promptly (1C) and opportunistic infection prophylaxis (1D).

8.3.1.2 Non-AIDS-defining malignancies

8.3.1.2.1 Anal cancer

- We recommend that all PLWH who are to be treated with chemoradiotherapy should start ART (1C) and opportunistic infection prophylaxis (1D).

8.3.1.2.2 Hodgkin lymphoma

- We recommend all PLWH and Hodgkin lymphoma should receive ART during chemotherapy (1A).

8.3.1.2.3 Other non-AIDS-defining cancers

- We suggest all PLWH who require chemotherapy or radical radiotherapy should receive concomitant ART and opportunistic infection prophylaxis unless contraindicated (level of evidence $2 \mathrm{C}$ ).

8.3.2 What to start

- We recommend that all potential interactions between ART, opportunistic infection prophylaxis and cancer therapy should be considered (1C).

- We suggest that all individuals with non-AIDS-defining malignancies who are due to start chemotherapy or radiotherapy should be started on ART unless contraindicated $(2 \mathrm{C}$ ).

8.3.3 Opportunistic infection prophylaxis in HIV-associated malignancy

- We recommend that all individuals with AIDS-defining malignancies should start ART immediately (1B).

- We suggest that all individuals with non-AIDS-defining malignancies who are due to start chemotherapy or radiotherapy should be started on ART immediately unless contraindicated (2C).

- We recommend herpes simplex virus (HSV) prophylaxis in PLWH with a history of HSV infection who are starting chemotherapy to reduce the incidence and severity of reactivations (1D).

- We recommend that individuals with antibodies against hepatitis B core antigen $(\mathrm{HBCAb})$ should be treated with prophylactic antivirals in line with BHIVA hepatitis guidelines (1B).

8.3.4 Other considerations from the BHIVA guidelines for HIV-associated malignancies

- We recommend that potential pharmacokinetic interactions between antiretrovirals and systemic anticancer therapy are checked prior to administration (with tools such as: www.hiv-druginteractions.org) (1C).

- We suggest avoiding ritonavir (or cobicistat)-boosted ART in HIV-positive individuals who are to receive cytotoxic chemotherapy agents that are metabolised by the CYP450 enzyme system (2C).

- We suggest avoiding atazanavir in HIV-positive individuals who are to receive irinotecan $(2 \mathrm{C})$.

- We suggest switching antiretroviral agents in HIV-positive patients who are to receive cytotoxic chemotherapy agents to avoid severe and/or overlapping toxicities (2C)

- Medicines reconciliation prior to chemotherapy to minimise potential pharmacokinetic interactions and overlapping toxicity should be undertaken by an experienced pharmacist (GPP)

8.4 HIV-associated neurocognitive impairment

8.4.2 When to start ART

8.4.2.1 Recommendation

- We recommend individuals with symptomatic HIV-associated neurocognitive disorders start ART immediately, irrespective of CD4 cell count (1C).

8.4.3 What to start with

8.4.3.1 Recommendations

- We recommend individuals with HIV-associated NC disorders start standard combination ART regimens (1C).

- We recommend avoiding efavirenz-containing regimens in individuals with HIV-associated NC disorders (1C)

8.4.4 Continuing or worsening NC impairment despite ART

8.4.4.1 Recommendations

Best practice management should include (GPP):
- Reassessment for confounding conditions.

- Assessment of CSF HIV RNA and genotyping of CSF HIV RNA.

- In subjects with detectable CSF HIV RNA, modifications to antiretroviral therapy should be based on paired plasma and CSF genotypic results.

8.5 Chronic kidney disease

8.5.1 When to start antiretroviral therapy

8.5.1.1 Recommendations

- We recommend individuals with HIV-associated nephropathy (HIVAN) start ART immediately irrespective of CD4 cell count (1C)

- We recommend individuals with end-stage kidney disease who are suitable candidates for renal transplantation start ART immediately (1C).

8.5.2 What to start

8.5.2.1 Recommendations

- We recommend against the use of ARV drugs that are potentially nephrotoxic in individuals with stages 3-5 CKD if acceptable alternative ARV agents are available (GPP).

- We recommend dose adjustment of renally cleared ARV drugs in individuals with reduced renal function (GPP).

8.5.3 Need to switch

8.5.3.1 Recommendations

- We recommend against continued use of tenofovir-DF and atazanavir in individuals with worsening renal function who have developed or are approaching CKD stages $3-5$ if acceptable alternative ARV agents are available (GPP)

8.6 Cardiovascular disease

8.6.2.1 Recommendation

- We suggest that the coronary heart disease (CHD) risk of HIV-positive adults of white ethnicity is estimated as per the BHIVA monitoring guidelines (www.bhiva.org/monitoring-guidelines.aspx) (2C).

8.6.4 What to start

8.6.4.1 Recommendations

In individuals with a high CVD risk:

- We recommend use of alternatives to fosamprenavir/r (1C) and lopinavir/r (1C)

- We suggest that atazanavir/r is the preferred PI (2C).

- We suggest avoiding abacavir (2C) and maraviroc if an acceptable alternative is available.

- First-line ARV therapy with tenofovir-DF plus (emtricitabine or lamivudine) with dolutegravir or raltegravir or rilpivirine (if viral load $<100,000$ copies $/ \mathrm{mL}$ ) are preferred first line regimens (GPP).

- Adverse effects on lipid parameters should be considered when selecting ARVs (GPP).

8.6.5 Modification of CVD risk factors

8.6.5.1 Recommendations

In patients with a high CVD risk:

- We recommend that traditional modifiable risk factors should be minimised; smoking cessation is of critical importance (1A).

- We suggest that that this should include switching ARVs to those with a more favourable metabolic profile but only where there is minimal risk of treatment failure $(2 \mathrm{C})$

8.7 Women

8.7.2 When to start

8.7.2.1 Recommendations

- We recommend therapy-naïve HIV-positive women who are not pregnant start ART (see Section 4.0) (1A).

8.7.3 What to start

8.7.3.1 Recommendations

- There are insufficient data to support specific recommendations for HIV-positive non-pregnant women. We therefore recommend therapynaïve HIV-positive women start ART as per general guidelines (1A).

- We recommend both HIV-positive women of childbearing potential and healthcare professionals who prescribe ART are conversant with the benefits and risks of ARV agents for both the health of the HIVpositive woman and for that of an unborn child (GPP).

- We recommend that potential pharmacokinetic interactions between ARVs, hormonal contraceptive agents and hormone replacement therapy are checked before administration (GPP) 
8.8 Mental health

8.8.1 Recommendations

- What to start: we recommend that efavirenz-containing regimens be avoided in individuals with a current or past history of depression, psychosis, suicidal ideation or attempted suicide, or at risk of self-harm (1C).

- Switching therapy: we recommend that efavirenz-containing regimens should be switched promptly to a viable alternative when PLWH present with depression, psychosis, suicidal ideation or attempted suicide, or self-harm (1C).

8.9 Adolescents

8.9.1 Recommendations for management of HIV, ART and sexual and reproductive health specifically for perinatally acquired HIV

- Avoid standard-dose (600 mg) efavirenz-based regimens in any young person $<50 \mathrm{~kg}$, with any history of mental health or psychological or neurocognitive problems.

8.10 Bone disease and antiretroviral therapy

8.10.2 When to start antiretroviral therapy

8.10.2.1 Recommendation

- We recommend that general recommendations for the timing of ART are followed in patients with, or at risk of osteoporosis (1D).
8.10.3 What to start

8.10.3.1 Recommendations

- We recommend against the use of tenofovir-DF disoproxil fumarate in individuals aged $>40$ years with osteoporosis, a history of fragility fracture, or a FRAX score of $>20 \%$ (major osteoporotic fracture) if acceptable alternative ARV agents are available (1B)

8.10.4 Switching treatment

8.10.4.1 Recommendations

- We recommend against continued use of tenofovir-DF in individuals $>40$ years who are diagnosed with osteoporosis, have sustained a fragility fracture, or have a FRAX score of $>20 \%$ (major osteoporotic fracture) if acceptable alternative ARV agents are available (1C).

8.11 Considerations for later life

8.11.2 When to start ART

8.11.2.1 Recommendation

- We recommend standard criteria are used to determine when to commence antiretroviral therapy in older PLWH (1C).

8.11.3 What to start

8.11.3.1 Recommendation:

- We recommend standard antiretroviral regimens are commenced in older PLWH (1C). 


\subsection{Involvement of people living with HIV in decision-making}

\subsection{Recommendations}

- We recommend PLWH are given the opportunity to be involved in making decisions about their treatment (GPP).

- Provision of treatment-support resources should include in-house, independent and community information providers and peer-support resources (GPP).

\subsection{Auditable outcomes}

- Percentage of PLWH who confirm they have been given the opportunity to be involved in making decisions about their treatment.

- Percentage of PLWH who have been offered signposting or referral to peer support or treatment advocacy services.

- Proportion of PLWH utilising peer support or treatment advocacy services.

\subsection{Rationale}

PLWH should be given the opportunity to be involved in making decisions about their treatment [1]. Studies show that trust, a good-quality relationship and good communication skills between doctor and PLWH are associated with better adherence and treatment outcomes in HIV and in other disease areas [2-6].

Studies have shown that beliefs about the necessity, efficacy and side effects of ART, the practicability of taking it, and ability to adhere to therapy, all affect adherence [7-9].

Before prescribing ART (treatment initiation or switching), clinicians should assess the individual's readiness to take therapy, including:

- Understanding of HIV therapy, and perceptions of personal need for ART;

- Concerns about taking ART or specific ARV drugs, including potential adverse effects;

- Concerns with possible adverse social consequences, such as disclosure or interference with lifestyle;

- Confidence that they will be able to adhere to the medication (self-efficacy);

- Psychological or neurocognitive issues that could impact on adherence;
- Socio-economic factors that could impact on adherence, including, but not limited to, poverty, housing, immigration status or domestic violence;

- Pregnancy or parenting plans.

Community advocacy and peer support, including clinic-based peer support, are helpful in supporting an individual's understanding and confidence around treatments, and may also help increase readiness to start therapy. Community organisations in the UK have been instrumental in providing a range of information resources for PLWH and peer-support services, including published and web-based information materials, telephone advice lines, treatment advocates and peer-support groups, working in collaboration with healthcare professionals. They are an important and essential adjunct to clinic-based services and are helpful in addressing the issues discussed below. Peer support is particularly important at diagnosis but there may be other crucial times whe peer support in particularly important such as starting/switching ART, disclosing to others or planning a family.

A number of factors may affect adherence, adverse effects and treatment outcomes, including social and cultural beliefs. Depression is significantly associated with low adherence [10,11] and some studies report an independent association between depression and mortality in people with HIV [12]. Adherence can be improved by treating depression [13], so all PLWH should be screened for depression before starting therapy, using simple screening tools such as the Arroll two question quick screen [14]. HIV-positive individuals should also be screened for anxiety and for cognitive impairment using validated tools (see the BHIVA guidelines for the routine investigation and monitoring of adult HIV-1-infected individuals 2011; www.bhiva.org/Guidelines.aspx).

Current problematic alcohol and recreational drug use are also associated with low adherence [15-17], although a history of injecting drug use, even current use, does not necessarily predict poor adherence [18]. PLWH should be asked about alcohol and recreational drug use and offered support to moderate or manage it if desired.

Conversely, adherence has been associated with positive experiences of quality of life such as having a meaningful life, feeling comfortable and well cared for, using time wisely, and taking time for important things [19]. 
Self-management skills and courses that teach these to PLWH have been associated with both improved adherence and better clinical outcomes in a number of studies [20-22]. It may be helpful to PLWH to inform them of these and other psychological support options locally available, in line with the BPS/BHIVA/MedFASH Standards for Psychological Support for Adults Living with HIV [23].

An individual's socio-economic status has a direct effect on adherence. For instance, a US study found that poverty had a direct effect on adherence, largely due to food insufficiency [24]. A 2010 report on poverty in people with HIV in the UK found that 1-in-6 people with HIV was living in extreme poverty, in many cases due to unsettled immigration status [25]. In addition, the ASTRA study revealed that after adjustment for demographic factors, increasing financial hardship, non-employment, non-homeownership, non-university education and lack of supportive network were associated with higher risk of virological rebound in ART-treated individuals [26]. Clinicians should be aware of the socio-economic status of HIV-positive individuals and refer to social support where necessary.

Clinicians should establish what level of involvement the HIV-positive individual would like and tailor their consultation style appropriately. They should also consider how to make information accessible and understandable to PLWH (e.g. with pictures, symbols, large print and different languages) [1], including addressing linguistic and cultural issues. Youth is consistently associated with lower adherence to ART, loss to follow-up and other negative healthcare behaviours [27] and some studies have found an independent association between poorer adherence and attendance and female gender [28], so information and consultation style should be age and gender appropriate for the individual.

If there is a question about an individual's capacity to make an informed decision, this should be assessed using the principles in the Mental Capacity Act 2005 [29].

PLWH presenting at the clinic may be at different stages of readiness to take therapy [30] and clinicians' first task is to assess their readiness, by means of open (rather than closed) questions. However, if an HIV-positive individual presents in circumstances that necessitate starting ART immediately, for example with an AIDS diagnosis or very low CD4 cell count, then doctors should prescribe ART and provide support for their adherence, especially through the first few weeks. Recognising symptoms that individuals attribute to ART side effects might avoid loss of adherence and deterioration of trust in the patient-provider relationship [31,32].
A 'perceptions and practicalities' approach should be used to tailor support to meet the needs of the individual, to identify both the perceptual factors (such as beliefs about ART) and practical factors (such as capacity and resources) influencing adherence [1,8].

In terms of preparing PLWH for potentially lifelong ART it is important to explain and discuss the rationale for ART, potential adverse effects, why adherence is essential and the implications of missed/stopped ART. Clear and appropriate explanations of the mechanisms of ART and how different drugs inhibit HIV replication may help clarify the rationale for combination therapy and why it is so important to take the drugs in the right way, at the right time. A review of the individual's social circumstances, support, occupation, social life, options to store ART, ability to follow any necessary food requirements, understanding of drug-drug interactions and where to seek advice are important. The impact of storing or taking ART on potential disclosure to others should be considered. Written information or signposting to online resources should be offered, as should access to peer support. The preparation required for ART will vary from one person to the next and the multidisciplinary team and/or repeat visits should be utilised as indicated.

Supporting PLWH requires good communication not just between clinician and the HIV-positive individual but also between all healthcare staff involved with their care, including those in their HIV services, their general practitioner (GP) and any clinicians involved in management of co-morbid conditions. PLWH should be offered copies of letters about them sent to their GP and other physicians. Disclosure of HIV status to the GP should be considered best practice and should always be discussed. However, an individual's decision not to disclose their status to their GP should be respected, subject to the clinician's duty to protect vulnerable individuals.

\subsection{References}

1 National Collaborating Centre for Primary Care. Medicines adherence: Involving patients in decisions about prescribed medicines and supporting adherence. NICE Clinical Guideline 76 (CG76). 2009. Available at: http:// guidance.nice.org.uk/CG76 (accessed August 2015).

2 Schneider J, Kaplan SH, Greenfield S et al. Better physicianpatient relationships are associated with higher reported adherence to antiretroviral therapy in patients with HIV infection. J Gen Intern Med 2004; 19: 1096-1103.

3 Kremer H, Ironson G. To tell or not to tell: why people with HIV share or don't share with their physicians whether they are taking their medications as prescribed. AIDS Care 2006; 18: 520-528. 
4 Roberts KJ. Physician-patient relationships, patient satisfaction, and antiretroviral medication Adherence among HIV-infected adults attending a public health clinic. AIDS Patient Care STDS 2002; 16: 43-50.

5 Owens DM, Nelson DK, Talley NJ. The irritable bowel syndrome: long-term prognosis and the physician-patient interaction. Ann Intern Med 1995; 122: 107-112.

6 Vermeire E, Hearnshaw H, Van Royen P, Denekens J. Patient adherence to treatment: three decades of research. A comprehensive review. J Clin Pharm Ther 2001; 26: 331342.

7 Horne R, Buick D, Fisher M et al. Doubts about necessity and concerns about adverse effects: identifying the types of beliefs that are associated with non-adherence to HAART. Int J STD AIDS 2004; 15: 38-44.

8 Horne R, Cooper V, Gellaitry G et al. Patients' perceptions of highly active antiretroviral therapy in relation to treatment uptake and adherence: the utility of the necessityconcerns framework. J Acquir Immune Defic Syndr 2007; 45: 334-341.

9 Gonzalez JS, Penedo FJ, Llabre MM et al. Physical symptoms, beliefs about medications, negative mood, and long-term HIV medication adherence. Ann Behav Med 2007; 34: 46-55.

10 Kacanek D, Jacobson DL, Spiegelman D et al. Incident depression symptoms are associated with poorer HAART adherence: a longitudinal analysis from the Nutrition for Healthy Living study. J Acquir Immune Defic Syndr 2010; 53: 266-272.

11 Gonzalez JS, Batchelder AW, Psaros C, Safren SA. Depression and HIV/AIDS treatment nonadherence: a review and meta-analysis. J Acquir Immune Defic Syndr 2011; 58: 181-187.

12 Lima VD, Geller J, Bangsberg DR et al. The effect of adherence on the association between depressive symptoms and mortality among HIV-infected individuals first initiating HAART. AIDS 2007; 21: 1175-1183.

13 Yun LW, Maravi M, Kobayashi JS et al. Antidepressant treatment improves adherence to antiretroviral therapy among depressed HIV-infected patients. J Acquir Immune Defic Syndr 2005; 38: 432-438.

14 Arroll B, Khin N, Kerse N. Screening for depression in primary care with two verbally asked questions: cross sectional study. BMJ 2003; 327: 1144-1146.

15 Hendershot CS, Stoner SA, Pantalone DW, Simoni JM. Alcohol use and antiretroviral adherence: review and meta-analysis. J Acquir Immune Defic Syndr 2009; 52: 180-202.

16 Reback CJ, Larkins S, Shoptaw S. Methamphetamine abuse as a barrier to HIV medication adherence among gay and bisexual men. AIDS Care 2003; 15: 775-785.
17 Halkitis PN, Kutnick AH, Borkowski T, Parsons JT. Adherence to HIV medications and club drug use among gay and bisexual men. XIV International AIDS Conference. July 2002. Barcelona, Spain [Abstract ThPeE7856].

18 Wood E, Hogg RS, Lima VD et al. Highly active antiretroviral therapy and survival in HIV-infected injection drug users. JAMA 2008; 300: 550-554.

19 Holzemer WL, Corless IB, Nokes KM et al. Predictors of selfreported adherence in persons living with HIV disease. AIDS Patient Care STDs 1999; 13: 185-197.

20 Smith SR, Rublein JC, Marcus C et al. A medication selfmanagement program to improve adherence to HIV therapy regimens. Patient Educ Couns 2003; 50: 187-199.

21 Gifford AL, Laurent DD, Gonzales VM et al. Pilot randomized trial of education to improve self-management skills of men with symptomatic HIV/AIDS. J Acquir Immune Defic Syndr Hum Retrovirol 1998; 18: 136-144.

22 Lorig KR, Sobel DS, Stewart AL et al. Evidence suggesting that a chronic disease self-management program can improve health status while reducing hospitalization: a randomized trial. Med Care 1999; 37: 5-14.

23 British Psychological Society, British HIV Association, Medical Foundation for AIDS and Sexual Health. Standards for psychological support for adults living with HIV. MedFASH; 2011. Available at: www.bhiva.org/ StandardsForPsychologicalSupport.aspx (accessed August 2015).

24 Kalichman SC, Grebler T. Stress and poverty predictors of treatment adherence among people with low-literacy living with HIV/AIDS. Psychosom Med 2010; 72: 810-816.

25 National AIDS Trust, Terrence Higgins Trust. Hardship Fund. Report: poverty and HIV 2006-2009. 2011. Available at: http://www.nat.org.uk/media/Files/Publications/Sep2010-Poverty-and-HIV-2006-2009.pdf (accessed August 2015).

26 Burch L, Smith C, Sherr L et al. Socioeconomic factors and virological rebound: a prospective UK cohort study. Conference on Retroviruses and Opportunistic Infections. March 2014. Boston, MA, USA [Abstract 560].

27 Fogarty L, Roter D, Larson S et al. Patient adherence to HIV medication regimens: a review of published and abstract reports. Patient Educ Couns 2002; 46: 93-108.

28 Tapp C, Milloy MJ, Kerr T et al. Female gender predicts lower access and adherence to antiretroviral therapy in a setting of free healthcare. BMC Infect Dis 2011; 11: 86.

29 General Medical Council. Guidance on good practice: consent guidance: capacity issues. 2010. Available at: www.gmc-uk.org/guidance/ethical_guidance/ consent_guidance_part3_capacity_issues.asp (accessed August 2015). 
30 Prochaska J0, DiClemente CC, Norcross JC. In search of how people change. Applications to addictive behaviors. Am Psychol 1992; 47: 1102-1114.

31 Duran S, Spire B, Raffi F et al. Self-reported symptoms after initiation of a protease inhibitor in HIV-infected patients and their impact on adherence to HAART. HIV Clin Trials 2001; 2: 38-45.
32 Preau M, Leport C, Villes V et al. Prevalence and predictors of deterioration of a trustful patient-provider relationship among HIV-infected persons treated with antiretroviral therapy. J Acquir Immune Defic Syndr 2008; 47: 467-471. 


\subsection{When to start}

\subsection{Chronic infection}

\subsubsection{Recommendations}

- We recommend people with HIV start ART (1A).

\subsubsection{Auditable outcomes}

- Proportion of diagnosed PLWH on ART.

- Proportion of PLWH not on ART where the rationale for this, and a discussion of the benefits of ART, has been documented at least annually.

\subsubsection{Rationale}

Until recently, BHIVA recommended that individuals with chronic HIV infection should start ART before the CD4 count fell to below 350 cells/ $\mu \mathrm{L}$ [1]. This recommendation was based on evidence from cohort studies that demonstrated an increased risk of disease progression in individuals who delayed ART until their CD4 count was below 350 cells $/ \mu \mathrm{L}$ and the absence of robust evidence from RCTs in which the intervention, comparator and populations were similar to our own setting [2-13].

Three trials have randomised people with a CD4 cell count $>350$ cells $/ \mu \mathrm{L}$ to start or to defer treatment. The HIV Prevention Trials Network (HPTN) 052 trial [14] randomised individuals to early (initiate ART at a CD4 cell count between 350 and 550 cells $/ \mu \mathrm{L}$ ) or deferred (initiate ART at a CD4 cell count below 250 cells $/ \mu \mathrm{L}$ ) ART. While the study reported a benefit to earlier ART initiation, those in the deferred comparator arm started treatment at a significantly lower CD4 cell count than recommended in the UK since 2008 [15]. This study is thus likely to overestimate the benefits of immediate treatment compared with starting at $<350$ cells $/ \mu \mathrm{L}$. A further study, the ANRS 12136 Temprano trial [16] presented results in early 2015. This study, conducted in the Ivory Coast, randomised persons with a CD4 cell count $<800$ cells $/ \mu \mathrm{L}$ to immediate ART or to receipt of ART adhering to WHO criteria for initiation of therapy (these criteria changed several times over the course of the trial). Again, while the trial results demonstrated a clinical benefit associated with earlier ART initiation, the median CD4 cell count at ART initiation in the comparator arm is likely to be much lower than would be considered clinically acceptable in the UK. Furthermore, the population considered in the trial (those in the Ivory Coast with a high incidence of tuberculosis) is substantially different to the UK setting.

More recently, preliminary results of the START study have been presented and published [17]. This study enrolled 4685 adults with CD4 cell counts above 500 cells $/ \mu \mathrm{L}$ (median CD4 cell count 651 cells $/ \mu \mathrm{L}$ ) in 35 countries, and randomised them to start ART immediately or to defer ART until the CD4 count fell below 350 cells $/ \mu \mathrm{L}$. The risk of developing AIDS, a serious non-AIDS event or of death (combined as the primary endpoint) was reduced by $57 \%$ in those who were randomised to start earlier, after a median follow-up period of 3 years. The results were similar in high-income when compared to low- and middle-income countries and driven mainly by a difference in rates of AIDS events, particularly TB and cancers. Immediate ART was not associated with higher risk of grade 4 events or unscheduled hospital admissions.

Data on virological efficacy, drug resistance and toxicity, and the results of substudies (examining effects on bone, neurological function, lung function and small arterial elasticity) and quality of life outcomes have not yet been reported. Nevertheless, these results demonstrate a benefit of ART at CD 4 counts of $>500$ cells $/ \mu \mathrm{L}$, and suggest that if individuals are able to commit to taking ART, it should be started, regardless of the CD4 count. Considerations when preparing an individual to start ART are addressed in Section 3.3. It is important to recognise that despite the significant reduction in relative risk of disease progression associated with early ART, the absolute risk of deferring ART was small. In this study, $4.1 \%$ of individuals in the deferred arm $v$ s. $1.5 \%$ in the immediate treatment arm experienced a serious illness over 3 years of follow-up. The absolute risk of deferring therapy should be considered when making individual decisions.

\subsubsection{References}

1 Williams I, Churchill D, Anderson J et al. British HIV Association guidelines for the treatment of HIV-1-positive adults with antiretroviral therapy 2012 (Updated November 2013). HIV Med 2014; 15 (Suppl 1): 1-85.

2 Braithwaite RS, Roberts MS, Chang CC et al. Influence of alternative thresholds for initiating HIV treatment on quality-adjusted life expectancy: a decision model. Ann Intern Med 2008; 148: 178-185.

3 Braithwaite RS, Roberts MS, Goetz MB et al. Do benefits of earlier antiretroviral treatment initiation outweigh harms for 
individuals at risk for poor adherence? Clin Infect Dis 2009; 48: 822-826.

4 Sterne JA, May M, Costagliola D et al. Timing of initiation of antiretroviral therapy in AIDS-free HIV-1-infected patients: a collaborative analysis of 18 HIV cohort studies. Lancet 2009; 373: 1352-1363.

5 Cain LE, Logan R, Robins JM et al. When to initiate combined antiretroviral therapy to reduce mortality and AIDS-defining illness in HIV-infected persons in developed countries: an observational study. Ann Intern Med 2011; 154: 509-515.

6 Jaen A, Esteve A, Miro JM et al. Determinants of HIV progression and assessment of the optimal time to initiate highly active antiretroviral therapy: PISCIS Cohort (Spain). $J$ Acquir Immune Defic Syndr 2008; 47: 212-220.

7 Kitahata MM, Gange SJ, Abraham AG et al. Effect of early versus deferred antiretroviral therapy for HIV on survival. $N$ Engl J Med 2009; 360: 1815-1826.

8 Plettenberg A, Brockmeyer NH, Haastert B et al. Impact of earlier HAART initiation on the immune status and clinical course of treated patients on the basis of cohort data of the German Competence Network for HIV/AIDS. Infection 2011; 39: $3-12$.

9 Writing Committee for the Cascade Collaboration. Timing of HAART initiation and clinical outcomes in human immunodeficiency virus type 1 seroconverters. Arch Intern Med 2011; 171: 1560-1569.

10 Cain LE, Robins JM, Lanoy E et al. When to start treatment? A systematic approach to the comparison of dynamic regimes using observational data. Int J Biostat 2010; 6 [Article 18].

11 Goldman DP, Juday T, Seekins D et al. Early HIV treatment in the United States prevented nearly 13,500 infections per year during 1996-2009. Health Aff (Millwood) 2014; 33: 362-369.

12 Olubajo B, Mitchell-Fearon K, Ogunmoroti 0. A comparative systematic review of the optimal CD4 cell count threshold for HIV treatment initiation. Interdiscip Perspect Infect Dis 2014; 2014: 625670.

13 Piroth L, Fournel I, Mahy S et al. A decision tree to help determine the best timing and antiretroviral strategy in HIV-infected patients. Epidemiol Infect 2011; 139: 18351844.

14 Cohen MS, Chen YQ, McCauley M et al. Prevention of HIV1 infection with early antiretroviral therapy. $N$ Engl J Med 2011; 365: 493-505.

15 Gazzard BG, Anderson J, Babiker A et al. British HIV Association Guidelines for the treatment of HIV-1-infected adults with antiretroviral therapy 2008. HIV Med 2008; 9: 563-608.

16 Danel C, Gabillard D, Le Carrou J et al. Early ART and IPT in HIV-infected African adults with high CD4 count
(Temprano Trial). Conference on Retroviruses and Opportunistic Infections. February 2015. Seattle, WA, USA [Abstract 115LB].

17 INSIGHT START Study Group. Initiation of antiretroviral therapy in early asymptomatic HIV infection. $N$ Engl J Med 2015; 373: 795-807.

\subsection{Individuals presenting with AIDS or a major} infection

\subsubsection{Recommendation}

- We recommend that individuals presenting with an AIDS-defining infection, or with a serious bacterial infection and a CD4 cell count $<200$ cells $/ \mu \mathrm{L}$, start ART within 2 weeks of initiation of specific antimicrobial chemotherapy (1B).

\subsubsection{Auditable outcome}

- Proportion of HIV-positive individuals presenting with an AIDS-defining infection or with a serious bacterial infection and a CD4 cell count $<200$ cells $/ \mu \mathrm{L}$ who are started on ART within 2 weeks of initiation of specific antimicrobial chemotherapy.

\subsubsection{Rationale}

This recommendation is largely based on the ACTG 5164 study that demonstrated fewer AIDS progressions/deaths and improved cost-effectiveness when ART was commenced within 14 days (median 12 days; IQR 9-13 days) compared with initiation after completion of treatment for the acute infection (median 45 days; IQR 41-55 days) $[1,2]$. Those with tuberculosis (TB) as the primary infection were excluded from this study, and the majority of individuals enrolled had Pneumocystis pneumonia. The patients were well enough to give informed consent and to take oral medications, and therefore the findings may not be generalisable to those who are severely unwell or who require intensive care. Previous observational data suggest a survival benefit for HIV-positive patients who are started on ART while in the intensive care unit [3,4], but the data are insufficient to make a recommendation in this group [3,4].

There was no increase in the incidence of immune reconstitution disorders or adverse events generally with early ART initiation in ACTG 5164 [1,5]. However, those with intracranial opportunistic infections may be more prone to severe immune reconstitution disorders with early ART initiation. Some data suggest that particular caution should be exercised with cryptococcal meningitis: two studies from sub-Saharan Africa have demonstrated an increased mortality with early ART 
initiation; however, both were in very different healthcare settings from the UK and one utilised antifungal regimens that would not be preferred [6,7]. The COAT study highlighted that those with an acellular CSF or with decreased levels of consciousness were at higher risk of death with early ART initiation [7]. It is important to note that immune reconstitution disorders can be difficult to diagnose and case definitions vary across studies. Those presenting with $\mathrm{TB}$ and malignancies are discussed in Section 8.

\subsubsection{References}

1 Zolopa A, Andersen J, Powderly W et al. Early antiretroviral therapy reduces AIDS progression/death in individuals with acute opportunistic infections: a multicenter randomized strategy trial. PLoS One 2009; 4: e5575.

2 Sax PE, Sloan CE, Schackman BR et al. Early antiretroviral therapy for patients with acute aids-related opportunistic infections: a cost-effectiveness analysis of ACTG A5164. HIV Clin Trials 2010; 11: 248-259.

3 Morris A, Wachter RM, Luce J et al. Improved survival with highly active antiretroviral therapy in HIV-infected patients with severe Pneumocystis carinii pneumonia. AIDS 2003; 17: 73-80.

4 Croda J, Croda MG, Neves A, De Sousa dos Santos S. Benefit of antiretroviral therapy on survival of human immunodeficiency virus-infected patients admitted to an intensive care unit. Crit Care Med 2009; 37: 1605-1611.

5 Grant PM, Komarow L, Andersen J et al. Risk factor analyses for immune reconstitution inflammatory syndrome in a randomized study of early vs. deferred ART during an opportunistic infection. PLoS One 2010; 5: e11416.

5 Makadzange AT, Ndhlovu CE, Takarinda K et al. Early versus delayed initiation of antiretroviral therapy for concurrent HIV infection and cryptococcal meningitis in sub-saharan Africa. Clin Infect Dis 2010; 50: 1532-1538.

7 Boulware DR, Meya DB, Muzoora C et al. Timing of antiretroviral therapy after diagnosis of cryptococcal meningitis. N Engl J Med 2014; 370: 2487-2498.

\subsection{Treatment of primary HIV infection}

\subsubsection{Recommendation}

- We recommend all individuals with suspected or diagnosed PHI are reviewed promptly by an HIV specialist and offered immediate ART (1B).

\subsubsection{Auditable outcomes}

- Proportion of individuals with PHI assessed by an HIV specialist within 2 weeks.

- Proportion of individuals with PHI offered immediate ART.

\subsubsection{Rationale}

Primary HIV infection (PHI) is defined as HIV infection within a maximum of 6 months from the estimated time of HIV transmission. It can be diagnosed based on laboratory test results in the setting of a clinical sexual history [1]. In the setting of the recent results from the START, TEMPRANO and HPTN052 trials, there is now no longer equipoise when counselling all individuals diagnosed with HIV; these studies showed clinical benefit to starting immediate ART over deferral [2-5].

In the context of PHI there are additional considerations to take into account when considering best management. PHI is a distinctive situation where oftensignificant symptoms consistent with seroconversion occur at a time of the stress of coming to terms with a new HIV diagnosis. Individuals diagnosed with PHI with low initial CD4 $\mathrm{T}$ cell counts [6,7], high plasma viral loads ( $>100,000$ copies HIV RNA) [8] and short test intervals (diagnosis within 12 weeks of a previous negative test) $[9,10]$ have a more rapid rate of disease progression than others without these features at PHI, and hence early ART initiation should be prioritised.

ART should be started only when the individual feels ready to do so. However, there are certain clinical presentations of PHI where expedited ART initiation should be recommended. We recommend starting ART as soon as possible for patients presenting with PHI meeting any one of the following criteria known to be associated with morbidity or very rapid disease progression:

- Neurological involvement (1D);

- Any AIDS-defining illness (1A);

- CD4 cell count $<350$ cells $/ \mu \mathrm{L}(1 \mathrm{C})$;

- PHI diagnosed within 12 weeks of a previous negative test (1C).

The pros and cons of early ART initiation with a view to long-term therapy should be clearly and sensitively presented to any individual diagnosed with PHI (Table 4.3.1). ART once started, should be considered as potentially lifelong due to the increased all-cause mortality observed from treatment interruption in the SMART study [11], which was seen regardless of nadir CD4 cell count. 
Table 4.3.1 The pros and cons of starting ART in PHI

\begin{tabular}{|c|c|}
\hline Pros of starting ART in PHI & Cons of starting ART in PHI \\
\hline $\begin{array}{l}\text { Enhanced probability of } \\
\text { immunological recovery to normal } \\
\text { levels [12-18] }\end{array}$ & $\begin{array}{l}\text { Ambivalence to ART at a time of } \\
\text { emotional challenges can risk poor } \\
\text { adherence and the development of } \\
\text { drug resistance }\end{array}$ \\
\hline $\begin{array}{l}\text { Individuals with recent HIV diagnosis } \\
\text { may feel comforted to know that } \\
\text { they are taking immediate control } \\
\text { of their infection with evidence to } \\
\text { support enhanced immunological } \\
\text { and virological benefits [19] }\end{array}$ & $\begin{array}{l}\text { Individuals with recently diagnosed } \\
\text { PHI may be in a particularly } \\
\text { vulnerable psychological state, and } \\
\text { thus ill-prepared to commit to } \\
\text { starting long-term treatment. }\end{array}$ \\
\hline \multicolumn{2}{|l|}{$\begin{array}{l}\text { Reduced risk of onward viral } \\
\text { transmission at a time of very } \\
\text { high viral load and consequent } \\
\text { high risk of transmission [19-24] }\end{array}$} \\
\hline \multicolumn{2}{|l|}{$\begin{array}{l}\text { Reduction in morbidity and more } \\
\text { rapid disease progression } \\
\text { associated with high viraemia [8] }\end{array}$} \\
\hline \multicolumn{2}{|l|}{$\begin{array}{l}\text { Recent data from the START, } \\
\text { TEMPRANO and HPTN052 trials } \\
\text { identify clinical benefit from } \\
\text { starting ART irrespective of CD4 } \\
\text { count [3-5] }\end{array}$} \\
\hline \multicolumn{2}{|l|}{$\begin{array}{l}\text { Earlier intervention within the first } \\
12 \text { weeks of diagnosis confers } \\
\text { enhanced immune recovery for } \\
\text { this group of individuals who } \\
\text { progress more rapidly if ART is } \\
\text { deferred [12-17] }\end{array}$} \\
\hline $\begin{array}{l}\text { Limitation of viral reservoir to } \\
\text { significantly below that seen when } \\
\text { treatment deferred [5] }\end{array}$ & \\
\hline
\end{tabular}

The rationale for immediate ART initiation amongst individuals diagnosed with PHI include:

- Preservation of immune function and CD4 T lymphocytes in terms of both total CD4 counts and the ratio of CD4:CD8 T cells, which reflects immune activation, inflammation and all-cause mortality that are otherwise impaired by uncontrolled viral replication, and are associated with survival in untreated individuals [13-19].

- Reduction in morbidity associated with high viraemia and profound CD4 cell depletion during acute infection [7-11].

- Reduction in the enhanced risk of onward transmission of HIV associated with PHI [21-26].

There is never likely to be a randomised controlled trial in PHI comparing immediate vs. deferred ART that is powered to a survival outcome, as such a study would require decades to accrue endpoints. Hence recommendations of best management of PHI are based on surrogate markers of mortality and CD4 cell count. Increasing evidence has identified both rapid and enhanced recovery of surrogate markers of the immune system [7-9] in terms of CD4 cell count [10] and CD4:CD8 ratio [11,12] for individuals initiating ART close to the time of HIV transmission compared to deferred ART initiation. A recent analysis demonstrated lower likelihood of achieving a normal CD4 cell count if treatment initiation was delayed more than 12 months after PHI; therefore, even outside the circumstances where prompt ART is advised, starting within 1 year of PHI diagnosis is advisable [18].

Immediate or expedited ART initiation for symptomatic seroconversion and those with very high plasma viral loads will additionally resolve clinical symptoms and limit the enhanced risk of onward viral transmission [1218]. Furthermore, earlier ART initiation has been shown to correspond with reduced measures of the latent pool of infected cells (viral reservoir) [27-29], the current barrier to HIV remission or cure [30,31]. We therefore recommend an expedited pathway of care for individuals diagnosed with PHI to ensure a clear and informed discussion of the pros and cons of immediate ART is provided to all individuals to support them making the correct treatment decision. An individual's readiness to start ART should be explored prior to commencing (see Section 3.3).

\subsubsection{References}

1 Rosenberg NE, Pilcher CD, Busch MP, Cohen MS. How can we better identify early HIV infections? Curr Opin HIV AIDS 2015; 10: 61-68.

2 Cohen MS, Shaw GM, McMichael AJ, Haynes BF. Acute HIV-1 Infection. N Engl J Med 2011; 364: 1943-1954.

3 INSIGHT START Study Group. Initiation of antiretroviral therapy in early asymptomatic HIV infection. $N$ Engl $J$ Med 2015; 373: 795-807.

4 Danel C, Moh R, Gabillard D et al. A trial of early antiretrovirals and isoniazid preventive therapy in Africa. Temprano ANRS Study Group. N Engl J Med 2015; 373: 808-822.

5 Cohen M, Chen Y, McCauley M et al. Final results of the HPTN 052 randomized controlled trial: antiretroviral therapy prevents HIV transmission. 8th IAS Conference on HIV Pathogenesis, Treatment and Prevention. July 2015. Vancouver, Canada.

6 Goujard C, Bonarek M, Meyer L et al. CD4 cell count and HIV DNA level are independent predictors of disease progression after primary HIV type 1 infection in untreated patients. Clin Infect Dis 2006; 42: 709-715.

7 Lodi S, Phillips A, Touloumi G et al. Time from human immunodeficiency virus seroconversion to reaching CD4+ cell count thresholds $<200,<350$, and $<500$ cells/mm(3): assessment of need following changes in treatment guidelines. Clin Infect Dis 2011; 53: 817-825.

8 Socias ME, Sued 0, Laufer $\mathrm{N}$ et al. Acute retroviral syndrome and high baseline viral load are predictors of 
rapid HIV progression among untreated Argentinean seroconverters. J Int AIDS Soc 2011; 14: 40.

9 CASCADE Collaboration. The relationships between the HIV test interval, demographic factors and HIV disease progression. Epidemiol Infect 2001; 127: 91-100.

10 Tyrer F, Walker AS, Gillett J, Porter K. The relationship between HIV seroconversion illness, HIV test interval and time to AIDS in a seroconverter cohort. Epidemiol Infect 2003; 131: 1117-1123.

11 El-Sadr WM, Lundgren J, Neaton JD et al. CD4 + countguided interruption of antiretroviral treatment. $N$ Engl $J$ Med 2006; 355: 2283-2296.

12 Rosenberg ES, Altfeld M, Poon SH et al. Immune control of HIV-1 after early treatment of acute infection. Nature 2000; 407: 523-526.

13 Macatangay BJ, Rinaldo CR. Preserving HIV-specific T cell responses: does timing of antiretroviral therapy help? Curr Opin HIV AIDS 2015; 10: 55-60.

14 Ananworanich J, Dube K, Chomont N. How does the timing of antiretroviral therapy initiation in acute infection affect HIV reservoirs? Curr Opin HIV AIDS 2015; 10: $18-28$.

15 Le T, Wright EJ, Smith DM et al. Enhanced CD4 + T-cell recovery with earlier HIV-1 antiretroviral therapy. $N$ Engl $J$ Med 2013; 368: 218-230.

16 Thornhill J, Inshaw J, Oomeer S et al. Enhanced normalisation of $\mathrm{CD} 4 / \mathrm{CD} 8$ ratio with early antiretroviral therapy in primary HIV infection. J Int AIDS Soc 2014; 17: 19480.

17 Kinloch S. Enhanced immune reconstitution with initiation of ART at HIV-1 seroconversion (PHI). 21st Annual BHIVA Conference. April 2015. Brighton, UK.

18 Okulicz JF, Le TD, Agan BK et al. Influence of the timing of antiretroviral therapy on the potential for normalization of immune status in human immunodeficiency virus 1-infected individuals. JAMA Intern Med 2015; 175: 88-99.

19 Parsons V, Porter K, Gilson R, Hart G. Attitudes and beliefs towards early ART initiation in MSM with primary HIV infection. J Int AIDS Soc 2014; 17: 19732.

20 Quinn TC, Wawer MJ, Sewankambo N et al. Viral load and heterosexual transmission of human immunodeficiency virus type 1. Rakai Project Study Group. $N$ Engl J Med 2000; 342: 921-929.

21 Powers KA, Ghani AC, Miller WC et al. The role of acute and early HIV infection in the spread of HIV and implications for transmission prevention strategies in Lilongwe, Malawi: a modelling study. Lancet 2011; 378: 256-268.

22 Pinkerton SD. Probability of HIV transmission during acute infection in Rakai, Uganda. AIDS Behav 2008; 12: 677-684.
23 Hollingsworth TD, Anderson RM, Fraser C. HIV-1 transmission, by stage of infection. J Infect Dis 2008; 198: 687-693.

24 Pao D, Fisher M, Hue S et al. Transmission of HIV-1 during primary infection: relationship to sexual risk and sexually transmitted infections. AIDS 2005; 19: 85-90.

25 Miller WC, Rosenberg NE, Rutstein SE, Powers KA. Role of acute and early HIV infection in the sexual transmission of HIV. Curr Opin HIV AIDS 2010; 5: 277-282.

26 Fisher M, Pao D, Brown AE et al. Determinants of HIV-1 transmission in men who have sex with men: a combined clinical, epidemiological and phylogenetic approach. AIDS 2010; 24: 1739-1747.

27 Williams JP, Hurst J, Stohr W et al. HIV-1 DNA predicts disease progression and post-treatment virological control. Elife 2014; 3: e03821.

28 Cheret A, Bacchus-Souffan C, Avettand-Fenoel V et al. Combined ART started during acute HIV infection protects central memory CD4 + T cells and can induce remission. $J$ Antimicrob Chemother 2015; 70: 2108-2120.

29 Laanani M, Ghosn J, Essat A et al. Impact of the Timing of Initiation of Antiretroviral Therapy During Primary HIV-1 Infection on the Decay of Cell-Associated HIV-DNA. Clin Infect Dis 2015; 60: 1715-1721.

30 Thornhill J, Fidler S, Frater J. Advancing the HIV cure agenda: the next 5 years. Curr Opin Infect Dis 2015; 28: 19.

31 Katlama C, Deeks SG, Autran B et al. Barriers to a cure for HIV: new ways to target and eradicate HIV-1 reservoirs. Lancet 2013; 381: 2109-2117.

\subsection{Impact of treatment on prevention of onward transmission}

\subsubsection{Recommendations}

- We recommend that ART is offered to all PLWH for the prevention of onward transmission (1A).

- We recommend the evidence that treatment with ART substantially lowers the risk of transmission is discussed with all PLWH (GPP).

- An assessment of the risk of transmission to others should be made at diagnosis and subsequent visits (GPP).

\subsubsection{Auditable outcome}

- Record in medical notes of all individuals about the discussion that treatment with ART lowers the risk of HIV transmission and perform an assessment of the current risk of transmission. 
The discussion should include the following:

- If the decision to start ART is being driven primarily by transmission risk it should be the HIV-positive individual's choice and must not be due to pressure from partners or others.

- The clinical benefits of ART at all CD4 cell counts should be emphasised.

- Individuals should be reassured about the relatively low risk of tolerability and toxicity issues with modern ART, and the option to switch ART if problems occur.

- Condoms, both male and female, continue to be recommended as protection from other sexually transmitted infections and unplanned pregnancy.

- There are risks associated with interrupting ART, and once started, it should generally be continued.

- Much of the evidence that ART lowers the risk of transmission mainly relates to vaginal sex. Recent data also show that ART greatly reduces the risk of transmission for anal sex, although the upper estimates for risk for anal sex are higher, and more data are needed; therefore, other prevention strategies, including condoms, should be discussed.

- High and consistent adherence to ART is required to maintain viral suppression and minimise transmission risk.

- Taking ART does not result in immediate complete viral suppression; it usually takes several months to achieve an undetectable viral load in blood.

\subsubsection{Rationale}

The potential effect of HIV treatment to reduce the risk of onward sexual transmission should be discussed with all PLWH as a part of safer sex messages in general. For individuals with a high CD4 cell count, the impact of treatment on the risk of transmission may be an additional factor to aid their decision-making. NHS England approved the use of ART for prevention in July 2015 [1].

The initial evidence base for treatment to reduce transmission was based on a number of cohort studies that found that transmission between heterosexual couples where the HIV-positive partner had an undetectable viral load on treatment was very rare or did not occur [2-6].

This was followed by good evidence from one randomised controlled trial (HPTN 052) [7] that showed ART can markedly reduce (by 96\%) the risk of transmission to HIV-negative partners. This is supported by the secondary outcomes of another trial [8] that also found a marked reduction in transmission from partners taking ART (by $92 \%)$. It is important to note that $97 \%$ of the couples in HPTN 052 were heterosexual and the Partners in Prevention study was conducted entirely with heterosexual couples. The evidence base thus relates mainly to the risk of transmission for vaginal sex in heterosexual couples.

More recently, data from the PARTNER study have demonstrated a protective effect of viral suppression in serodifferent couples where the HIV-positive person is on suppressive ART (viral load less than 200 copies $/ \mathrm{mL}$ ). After 16,800 and 28,000 condomless sex acts in MSM and heterosexual couples respectively, there were no cases of HIV transmission when 86 would have been expected based on previous incidence studies [9].

Condoms should still be recommended to protect from other sexually transmitted infections, and to lower further any residual risk of transmission.

PLWH should be informed that taking ART does not result in immediate viral suppression. Most individuals commencing ART achieve viral suppression by 36 months; integrase inhibitors are characterised by more rapid viral suppression with most individuals achieving an undetectable viral load by 1-3 months [10-12]. PLWH should also be informed about the possibility of virological failure leading to transmission of HIV. Decisions on condom use and safer sex should always be based on a recent viral load test result and not on an assumption that taking ART implies non-infectiousness.

Suppressive ART expands the choices available to serodifferent heterosexual people wishing to conceive. Sex without condom use around the time of ovulation (timed UPSI) carries very little if any risk provided the HIV-positive partner has a durably suppressed viral load. Preexposure prophylaxis, although recommended in some guidelines [13], is likely to add very little as risk reduction in this scenario. Details of the use of ART to prevent mother-to-child transmission are covered in the BHIVA guidelines for the management of HIV infection in pregnant women [14]. Sperm washing is not recommended in the context of viral suppression [15].

\subsubsection{References}

1 NHS England. NHS England announces annual investment decisions for certain specialised services. 2015. Available at: http://www.england.nhs.uk/2015/07/02/annual-investmentdecisions/ (accessed August 2015).

2 Castilla J, Del Romero J, Hernando V et al. Effectiveness of highly active antiretroviral therapy in reducing heterosexual transmission of HIV. J Acquir Immune Defic Syndr 2005; 40: 96-101.

3 Del Romero J, Castilla J, Hernando V et al. Combined antiretroviral treatment and heterosexual transmission of HIV-1: cross sectional and prospective cohort study. BMJ 2010; 340: c2205.

4 Melo M, Varella I, Nielsen K et al. Demographic characteristics, sexual transmission and CD4 progression 
among heterosexual HIV-serodiscordant couples followed in Porto Alegre, Brazil. 16th International AIDS Conference. August 2006. Toronto, Canada [Abstract TUPE0430].

5 Attia S, Egger M, Muller M et al. Sexual transmission of HIV according to viral load and antiretroviral therapy: systematic review and meta-analysis. AIDS 2009; 23: 13971404.

6 Barreiro P, del Romero J, Leal M et al. Natural pregnancies in HIV-serodiscordant couples receiving successful antiretroviral therapy. J Acquir Immune Defic Syndr 2006; 43: 324-326.

7 Cohen MS, Chen YQ, McCauley M et al. Prevention of HIV1 infection with early antiretroviral therapy. $N$ Engl J Med 2011; 365: 493-505.

8 Donnell D, Baeten JM, Kiarie J et al. Heterosexual HIV-1 transmission after initiation of antiretroviral therapy: a prospective cohort analysis. Lancet 2010; 375: 2092-2098.

9 Rodger A, Bruun T, Cambiano V et al. HIV transmission risk through condomless sex if HIV+ partner on suppressive ART: PARTNER study. Conference on Retroviruses and Opportunistic Infections. March 2014. Boston, MA, USA [Abstract 153LB].

10 Walmsley SL, Antela A, Clumeck N et al. Dolutegravir plus abacavir-lamivudine for the treatment of HIV-1 infection. $N$ Engl J Med 2013; 369: 1807-1818.

11 Clumeck N, Molina JM, Henry K et al. A randomized, double-blind comparison of single-tablet regimen elvitegravir/cobicistat/emtricitabine/tenofovir DF vs ritonavir-boosted atazanavir plus emtricitabine/tenofovir DF for initial treatment of HIV-1 infection: analysis of week 144 results. J Acquir Immune Defic Syndr 2014; 65: e121124.

12 Wohl DA, Cohen C, Gallant JE et al. A randomized, doubleblind comparison of single-tablet regimen elvitegravir/ cobicistat/emtricitabine/tenofovir DF versus single-tablet regimen efavirenz/emtricitabine/tenofovir DF for initial treatment of HIV-1 infection: analysis of week 144 results. $J$ Acquir Immune Defic Syndr 2014; 65: e118-120.

13 Fakoya A, Lamba H, Mackie N et al. British HIV Association, BASHH and FSRH guidelines for the management of the sexual and reproductive health of people living with HIV infection 2008. HIV Med 2008; 9: 681-720.

14 de Ruiter A, Taylor GP, Clayden P et al. British HIV Association guidelines for the management of HIV infection in pregnant women 2012 (2014 interim review). HIV Med 2014; 15 (Suppl 4): 1-77.

15 National Institute for Health and Care Excellence. Fertility: Assessment and treatment for people with fertility problems. NICE guidelines (CG156). 2013. Available at: https:// www.nice.org.uk/guidance/cg156/ifp/chapter/hiv-hepatitisb-and-hepatitis-c (accessed August 2015). 


\subsection{What to start}

\subsection{Summary of recommendations}

- We recommend that therapy-naïve PLWH start ART containing two nucleoside reverse transcriptase inhibitors (NRTIs) plus one of the following: ritonavirboosted protease inhibitor (PI/r), non-nucleoside reverse transcriptase inhibitor (NNRTI) or integrase inhibitor (INI) (1A).

Table 5.1.1 Summary recommendations for choice of ART

\begin{tabular}{|c|c|c|}
\hline & Preferred & Alternative \\
\hline NRTI backbone & $\begin{array}{c}\text { Tenofovir-DF and } \\
\text { emtricitabine }\end{array}$ & $\begin{array}{l}\text { Abacavir and } \\
\text { lamivudine }{ }^{\mathrm{a}, \mathrm{b}}\end{array}$ \\
\hline $\begin{array}{l}\text { Third agent (alphabetical } \\
\text { order) }\end{array}$ & $\begin{array}{l}\text { Atazanavir/r } \\
\text { Darunavir/r } \\
\text { Dolutegravir } \\
\text { Elvitegravir/c }^{c} \\
\text { Raltegravir } \\
\text { Rilpivirine }^{\mathrm{d}}\end{array}$ & Efavirenz \\
\hline
\end{tabular}

/r: boosted with ritonavir; /c: boosted with cobicistat. ${ }^{a}$ Abacavir is contraindicated if an individual is HLAB*57:01 positive.

${ }^{\mathrm{b}}$ Use recommended only if baseline viral load is $<100,000$ copies/mL except when initiated in combination with dolutegravir in which case abacavir/lamivudine can be used at any baseline viral load.

${ }^{\mathrm{C}}$ Tenofovir-DF/emtricitabine/elvitegravir/c fixed-dose combination should not be initiated in individuals with creatinine clearance $<70 \mathrm{~mL} / \mathrm{min}$.

${ }^{\mathrm{d}}$ Use recommended only if baseline viral load is $<100,000$ copies/mL.

NB: The viral load advice for abacavir/lamivudine and rilpivirine applies only to initiating these agents in individuals with a detectable viral load - when these agents are used as a switch option in the context of viral load suppression the baseline viral load can be disregarded.

\subsubsection{Summary of auditable outcomes}

- Proportion of therapy-naïve individuals starting ART containing preferred or alternative agents.

- Proportion of individuals with undetectable viral load at 6 months and at 12 months after starting ART.
- Proportion of individuals who switch therapy in the first 6 and 12 months.

\subsection{Introduction}

For the 'which NRTI backbone' and 'which third agent' questions, evidence profiles and summary of findings tables were constructed to assess quality of evidence across predefined treatment outcomes (Appendix 3). Evidence from randomised controlled trials and systematic reviews was identified from a literature search (Appendix 2). Outcomes were scored and ranked (critical, important, not important) by members of the writing group. The following were ranked as critical outcomes: viral suppression at week 48/96, proportion with virological failure, proportion developing resistance, proportion discontinuing for adverse events, proportion with grade $3 / 4$ adverse events.

Treatments were compared and differences in critical outcomes assessed. Where there were differences, consensus opinion was sought to determine whether the difference in size of effect was above the threshold for clinical decision-making. If conflicting differences were detected, the balance of outcomes was based on consensus opinion of the writing group.

A treatment was defined as preferred or alternative to indicate strong or conditional recommendations with each decision based on the assessment of critical outcomes and the balance of desirable and undesirable effects in a general ART-naïve population. 'Preferred' indicates a strong recommendation that most clinicians and PLWH would want to follow unless there is a clear rationale not to do so. 'Alternative' indicates a conditional recommendation and is an acceptable treatment option for some PLWH and might be, in selected individuals, the preferred option.

Factors including potential side effects, co-morbidities, individual preference and drug interactions need to be taken into account when selecting an ART regimen, and may include both preferred and alternative treatment options.

For guidance on assessment of PLWH before initiation of ART and monitoring individuals on ART, the reader should consult the BHIVA guidelines for the routine investigation and monitoring of adult HIV-1-infected individuals (www.bhiva.org/monitoring-guidelines.aspx). 


\subsection{Which nucleoside reverse transcriptase inhibitor backbone}

\subsubsection{Recommendations}

- We recommend therapy-naïve individuals start combination ART containing tenofovir-DF and emtricitabine as the preferred NRTI backbone (1A).

- We suggest abacavir and lamivudine is an acceptable alternative NRTI backbone in therapy-naïve individuals. In those with a baseline viral load $>100,000$ copies $/ \mathrm{mL}$, it should be used with caution if there are clinical reasons to prefer over tenofovir-DF and emtricitabine (2A).

The caution regarding baseline viral load does not apply if abacavir/lamivudine is used with dolutegravir (2A).

- Abacavir must not be used in individuals who are HLA-B*57:01-positive (1A).

\subsubsection{Rationale}

The evidence and rationale for which NRTI backbone to use for initial therapy has not changed since the last iteration of the guidelines in 2012 [1]. Three randomised controlled trials have compared tenofovir-DF/ emtricitabine with abacavir/lamivudine as the NRTI backbone in combination with different third agents: atazanavir/r or efavirenz [2-6], efavirenz [7-9] and lopinavir/r [10].

Assessment of virological efficacy as a critical outcome was complicated by different definitions across the three studies. By GRADE analysis (see Appendix 3), there was no difference in rates of virological suppression at 48 weeks or 96 weeks but the analysis excluded the largest of the three trials (ACTG 5202) and the quality of evidence for this outcome was assessed as low or very low. Assessment of the risk of protocol-defined virological failure at 48 weeks favoured tenofovir-DF/emtricitabine (relative risk [RR] 0.76, 95\% confidence interval [CI] 0.53-1.07), although the effect was not statistically significant and heterogeneity in the analysis was relatively high. Assessment of protocol-defined virological failure at 96 weeks showed a significant difference favouring tenofovir-DF/emtricitabine (RR 0.73, 95\% CI 0.59-0.92). Data were only available from one study [2] for this analysis; however, this was by far the largest of the three trials and the quality of evidence assessment for this outcome was rated as high. The difference in virological failure was assessed to be large enough to be above the clinical threshold for decision-making. The difference equates to a number needed to treat for 1 year to prevent one case of virological failure of approximately 20 .
The results of ACTG 5202 [2-4] are complicated by early withdrawal of those individuals receiving abacavir/ lamivudine with a baseline viral load $>100,000$ copies $/ \mathrm{mL}$ at the recommendation of the Data and Safety Monitoring Board owing to significantly inferior performance. No difference in virological efficacy between the tenofovir$\mathrm{DF} /$ emtricitabine and abacavir/lamivudine arms was seen in those in the lower viral load stratum (baseline viral load $<100,000$ copies $/ \mathrm{mL}$ ). The subsequent 96-week analysis, after discontinuation of those subjects in the higher viral load stratum, may therefore underestimate the difference between the two backbones. HLA-B*57:01 screening was not routine in ACTG 5202 and potentially this may have influenced some of the safety endpoints, but appears not to have influenced the primary virological outcome. In the higher viral load stratum the number of study participants with suspected hypersensitivity reactions was equal between both arms and virological failure in these individuals was infrequent.

With regard to the assessment of the other critical and important outcomes, no difference was shown between tenofovir-DF/emtricitabine and abacavir/lamivudine. No data were available to assess quality of life outcomes. For grade $3 / 4$ adverse events (all) and grade 3/4 alanine transaminase/aspartate transaminase elevation there were trends that favoured tenofovir-DF/emtricitabine (see Appendix 3).

Although the rate of drug resistance was not different between the NRTI backbones, the number developing drug resistance was higher numerically in those receiving abacavir/lamivudine, given the higher rate of virological failure.

Bone mineral density outcomes significantly favoured abacavir/lamivudine.

Given the favourable virological outcomes of tenofovir$\mathrm{DF} / \mathrm{em}$ tricitabine compared with abacavir/lamivudine and the lack of other significant differences in critical and important adverse event outcomes, tenofovir-DF/emtricitabine is recommended as the preferred NRTI backbone of choice. Abacavir/lamivudine is an acceptable alternative option in individuals with a baseline viral load $<100,000$ copies $/ \mathrm{mL}$ (other than in combination with dolutegravir when abacavir/lamivudine can be initiated regardless of baseline viral load), but must only be used after ensuring HLA-B*57:01 is negative.

When selecting an NRTI backbone, factors such as potential side effects, co-morbidities, individual preference and cost should also be considered. Observational studies have variably reported associations between abacavir and cardiovascular disease (CVD) [11-14], and tenofovir-DF may cause renal disease [15]. These aspects are discussed in more detail in Section 8. However, based on 
the balance of current evidence we suggest abacavir is not used in individuals at high risk of CVD (see Section 8.6 Cardiovascular disease) and tenofovir-DF is not used in people with stage 3-5 chronic kidney disease (CKD) or at high risk of progression of CKD (see Section 8.5 Chronic kidney disease) if acceptable alternative antiretroviral agents are available.

\subsubsection{Not recommended}

We believe there is no routine role for other NRTI backbones in the treatment of ART-naïve patients. Zidovudine/lamivudine may be considered in certain specific circumstances (e.g. pregnancy [16]) but should not be given routinely due to the proven association with mitochondrial toxicity, particularly lipoatrophy, with zidovudine. There is no place for the use of stavudine- or didanosine-containing regimens as initial therapy, due to the associations with significant mitochondrial and hepatic toxicities.

\subsubsection{Lamivudine vs. emtricitabine}

The nucleoside backbone tenofovir-DF/lamivudine has not been well studied in randomised trials. This combination warrants further review of evidence as the lower costs associated with generic medications may make this an attractive combination in the future. There are a number of theoretical advantages in using emtricitabine rather than lamivudine, including:

- Emtricitabine triphosphate (TP) has a longer intracellular half-life [17];

- Emtricitabine TP is incorporated more efficiently than lamivudine TP into proviral deoxyribonucleic acid (DNA) [18];

- Greater in vitro potency [19].

Extensive review of the literature between 2000 and 2015 identified seven studies in ART-naive individuals that included randomisation to a lamivudine- or emtricitabine-containing backbone. In two of these lamivudine $v s$. emtricitabine was the only variable [20,21]. However, neither of these studies has been published and therefore have not been subject to formal peer review. One study, performed in Zambia, randomised 332 subjects to fixeddose combinations of either tenofovir-DF/lamivudine/efavirenz or tenofovir-DF/emtricitabine/efavirenz. Virological suppression rates at 48 weeks were similar (85.3\% vs. $90.1 \%)$ but due to wide $97.5 \%$ confidence limits (-28.1 to $18.4 \%$ ), non-inferiority of the lamivudine-containing regimen was not demonstrated. The other unpublished study in ART-naïve individuals was a double-blind comparison of lamivudine or emtricitabine with stavudine and either efavirenz or nevirapine with virological failure defined as a viral load $>400$ copies $/ \mathrm{mL}$ on consecutive visits. The probability of virological failure was reported as $10 \%$ with either agent, although a significantly lower rate of $\mathrm{M} 184 \mathrm{~V}$ mutation development on failure was reported in the emtricitabine-containing regimen [21]. A significantly lower rate of M184V mutation development on failure was reported in the emtricitabine-containing regimen. Of the remaining five studies at least one other component, additional to lamivudine or emtricitabine, differed, therefore prohibiting direct comparison. Three studies compared abacavir/lamivudine with tenofovir-DF/ emtricitabine $[3,7,10]$, one compared zidovudine/lamivudine with tenofovir-DF/emtricitabine [22], one compared zidovudine/lamivudine/efavirenz vs. tenofovir-DF/emtricitabine efavirenz vs. didanosine/emtricitabine/atazanavir [23]. A randomised comparison of 440 patients stable on therapy with suppressed viral load substituting lamivudine for emtricitabine showed no difference in virological failure at 48 weeks [24]. However, this is of limited applicability to the question of therapy in ART-naive patients since 'stable switch' studies are inherently biased in selecting patients who are already doing well on treatment. Of note, no trials using abacavir/emtricitabine as a backbone have been performed.

A number of observational studies and retrospective analyses of randomised controlled trials have sought to find clinical correlates of the pharmacological differences between lamivudine and emtricitabine. In general, these studies focus on the development of resistance, as evidenced by the emergence of the M184I/V mutation on treatment failure. Where data on resistance were available, none of the individual randomised controlled trial trials showed significant differences between lamivudineand emtricitabine-containing arms. However, a pooled analysis [25] of three trials of lamivudine or emtricitabine with another NRTI plus efavirenz showed significantly less emergent M184V on emtricitabine (odds ratio [OR] 0.32, $P=0.02$ ) when considering the whole treated population. However, the interpretation of this result is complicated by the use, with lamivudine, of nucleoside analogues that are no longer recommended due to toxicity issues, making it difficult to confidently exclude differences in adherence contributing to the development of resistance. A sub-analysis in this same paper compared the tenofovir-DF/lamivudine/efavirenz arm from one randomised controlled trial with tenofovir-DF/emtricitabine/ efavirenz from another and again found a significantly lower frequency of M184V/I [19]; this type of cross-trial comparison must, however, be interpreted with caution. A WHO appraisal on the interchangeability of lamivudine and emtricitabine concluded that the clinical and virological efficacy and safety of lamivudine and emtricitabine 
are comparable but the development of the M184V/I mutation is associated to a greater extent with the use of a lamivudine- rather than an emtricitabine-containing regimen [26]. They also stated, however, that the clinical and public health implications of this difference are not clear.

Cohort studies investigating this same question are subject to a number of confounders and have given mixed results. In general, they have shown significantly lower M184I/V development with the use of emtricitabine [27-29] but most have not restricted the analysis to individuals starting their first treatment regimen and have included a diverse range of other antiretroviral agents, so the relevance to recommendations for first-line therapy is open to question. A large UK CHIC analysis, including 5455 HIV-positive individuals, directly compared tenofovir-DF/lamivudine with tenofovir-DF/emtricitabine and found no significant difference in the emergence of M184V/I. This study was not restricted to treatment-naive individuals [30]. On the other hand, a cohort study in the Netherlands of 4740 individuals was restricted to those starting first-line therapy with tenofovir-DF/lamivudine or tenofovir-DF/emtricitabine together with an NNRTI [31]. This study showed higher rates of virological failure for the lamivudine-containing regimens $(\mathrm{OR}=1.78$, $P=0.16$ given with efavirenz) but did not adjust for year of HAART initiation in the multivariable analysis. This is important as lamivudine-containing regimens were started in significantly earlier years than emtricitabinecontaining regimens and there has been a well-documented improvement in virological outcomes as the use of HIV therapy has evolved; this and other unidentified confounders may limit the validity of the results [32].

It is clear that the evidence concerning this question is mixed and of variable quality, leaving open the question of whether lamivudine and emtricitabine are interchangeable in first-line therapy. The paucity of sufficiently high-quality evidence addressing this question means that tenofovir-DF/lamivudine cannot be clearly recommended as an alternative nucleoside backbone when initiating first-line therapy.

If cost pressures become such that co-formulation is no longer a major driving factor in antiretroviral choice, then it will be reasonable to revisit this recommendation.

\subsubsection{References}

1 Williams I, Churchill D, Anderson J et al. British HIV Association guidelines for the treatment of HIV-1-positive adults with antiretroviral therapy 2012 (Updated November 2013). HIV Med 2014; 15 (Suppl 1): 1-85.

2 Sax PE, Tierney C, Collier AC et al. Abacavir/lamivudine versus tenofovir DF/emtricitabine as part of combination regimens for initial treatment of HIV: final results. $J$ Infect Dis 2011; 204: 1191-1201.

3 Sax PE, Tierney C, Collier AC et al. Abacavir-lamivudine versus tenofovir-emtricitabine for initial HIV-1 therapy. $N$ Engl J Med 2009; 361: 2230-2240.

4 Daar ES, Tierney C, Fischl MA et al. Atazanavir plus ritonavir or efavirenz as part of a 3-drug regimen for initial treatment of HIV-1. Ann Intern Med 2011; 154: 445-456.

5 McComsey GA, Kitch D, Daar ES et al. Bone mineral density and fractures in antiretroviral-naive persons randomized to receive abacavir-lamivudine or tenofovir disoproxil fumarate-emtricitabine along with efavirenz or atazanavirritonavir: Aids Clinical Trials Group A5224s, a substudy of ACTG A5202. J Infect Dis 2011; 203: 1791-1801.

6 McComsey GA, Kitch D, Sax PE et al. Peripheral and central fat changes in subjects randomized to abacavir-lamivudine or tenofovir-emtricitabine with atazanavir-ritonavir or efavirenz: ACTG Study A5224s. Clin Infect Dis 2011; 53: 185-196.

7 Post FA, Moyle GJ, Stellbrink HJ et al. Randomized comparison of renal effects, efficacy, and safety with oncedaily abacavir/lamivudine versus tenofovir/emtricitabine, administered with efavirenz, in antiretroviral-naive, HIV-1infected adults: 48-week results from the ASSERT study. $J$ Acquir Immune Defic Syndr 2010; 55: 49-57.

8 Stellbrink HJ, Orkin C, Arribas JR et al. Comparison of changes in bone density and turnover with abacavirlamivudine versus tenofovir-emtricitabine in HIV-infected adults: 48-week results from the ASSERT study. Clin Infect Dis 2010; 51: 963-972.

9 Moyle GJ, Stellbrink HJ, Compston J et al. Comparison of bone and renal toxicities in the ASSERT study: final 96 week results from a prospective randomized safety trial. Antivir Ther 2010; 15 (Suppl 4): A19.

10 Smith KY, Patel P, Fine D et al. Randomized, double-blind, placebo-matched, multicenter trial of abacavir/lamivudine or tenofovir/emtricitabine with lopinavir/ritonavir for initial HIV treatment. AIDS 2009; 23: 1547-1556.

11 Bedimo RJ, Westfall A0, Drechsler $\mathrm{H}$ et al. Abacavir use and risk of acute myocardial infarction and cerebrovascular events in the highly active antiretroviral therapy era. Clin Infect Dis 2011; 53: 84-91.

12 Obel N, Farkas DK, Kronborg G et al. Abacavir and risk of myocardial infarction in HIV-infected patients on highly active antiretroviral therapy: a population-based nationwide cohort study. HIV Med 2010; 11: 130-136.

13 Palella F, Althoff K, Zhang J et al. Abacavir use and risk for myocardial infarction in the NA-ACCORD. Conference on Retroviruses and Opportunistic Infections. February 2015. Seattle, WA, USA [Abstract 749LB].

14 Worm SW, Sabin C, Weber R et al. Risk of myocardial infarction in patients with HIV infection exposed to specific 
individual antiretroviral drugs from the 3 major drug classes: the data collection on adverse events of anti-HIV drugs (D:A:D) study. J Infect Dis 2010; 201: 318-330.

15 Yombi JC, Pozniak A, Boffito M et al. Antiretrovirals and the kidney in current clinical practice: renal pharmacokinetics, alterations of renal function and renal toxicity. AIDS 2014; 28: 621-632.

16 de Ruiter A, Taylor GP, Clayden P et al. British HIV Association guidelines for the management of HIV infection in pregnant women 2012 (2014 interim review). HIV Med 2014; 15 (Suppl 4): 1-77.

17 Wang LH, Begley J, St Claire RL, 3rd et al. Pharmacokinetic and pharmacodynamic characteristics of emtricitabine support its once daily dosing for the treatment of HIV infection. AIDS Res Hum Retroviruses 2004; 20: 1173-1182.

18 Feng JY, Shi J, Schinazi RF, Anderson KS. Mechanistic studies show that (-)-FTC-TP is a better inhibitor of HIV-1 reverse transcriptase than 3TC-TP. FASEB $J$ 1999; 13: 1511-1517.

19 Drogan D, Rauch P, Hoffmann D et al. The antiretroviral potency of emtricitabine is approximately 3 -fold higher compared to lamivudine in dual human immunodeficiency virus type 1 infection/competition experiments in vitro. Antiviral Res 2010; 86: 312-315.

20 Mulenga L, Muwango A, Moyo C et al. Efficacy of tenofovir disoproxil fumarate/emtricitabine and tenofovir disoproxil both in combination with efavirenz in antiretroviral-naïve, HIV-1-infected Zambians. 7th IAS Conference on HIV Pathogenesis, Treatment and Prevention. July 2013. Kuala Lumpur, Malaysia [Abstract TULBPE18].

21 Sanne I, van der Horst C, Shaw A et al. Two randomized, controlled, equivalence trials of emtricitabine (FTC) to lamivudine (3TC). XIV International AIDS Conference. July 2002. Barcelona, Spain [Abstract 4432].

22 Gallant JE, Winston JA, DeJesus E et al. The 3-year renal safety of a tenofovir disoproxil fumarate vs. a thymidine analogue-containing regimen in antiretroviral-naive patients. AIDS 2008; 22: 2155-2163.

23 Campbell TB, Smeaton LM, Kumarasamy N et al. Efficacy and safety of three antiretroviral regimens for initial treatment of HIV-1: a randomized clinical trial in diverse multinational settings. PLoS Med 2012; 9: e1001290.

24 Benson CA, van der Horst C, Lamarca A et al. A randomized study of emtricitabine and lamivudine in stably suppressed patients with HIV. AIDS 2004; 18: 2269-2276.

25 McColl DJ, Margot N, Chen SS et al. Reduced emergence of the M184V/I resistance mutation when antiretroviral-naive subjects use emtricitabine versus lamivudine in regimens composed of two NRTIs plus the NNRTI efavirenz. HIV Clin Trials 2011; 12: 61-70.

26 World Health Organization. Technical update on treatment optimization: Pharmacological equivalence and clinical iterchangeability of lamivudine and emtricitabine: a review of current literature. 2012. Available at: http://apps.who.int/ iris/bitstream/10665/70936/1/9789241503815_eng.pdf

(accessed August 2015).

27 Marcelin AG, Charpentier C, Wirden M et al. Resistance profiles of emtricitabine and lamivudine in tenofovircontaining regimens. J Antimicrob Chemother 2012; 67: 1475-1478.

28 Maserati R, De Silvestri A, Uglietti A et al. Emerging mutations at virological failure of HAART combinations containing tenofovir and lamivudine or emtricitabine. AIDS 2010; 24: 1013-1018.

29 Svicher V, Alteri C, Artese A et al. Different evolution of genotypic resistance profiles to emtricitabine versus lamivudine in tenofovir-containing regimens. $J$ Acquir Immune Defic Syndr 2010; 55: 336-344.

30 Bulteel N, Bansi-Matharu L, Churchill D et al. The emergence of drug resistant HIV variants at virological failure of HAART combinations containing efavirenz, tenofovir and lamivudine or emtricitabine within the UK Collaborative HIV Cohort. J Infect 2014; 68: 77-84.

31 Rokx C, Fibriani A, van de Vijver DA et al. Increased virological failure in naive HIV-1-infected patients taking lamivudine compared with emtricitabine in combination with tenofovir and efavirenz or nevirapine in the Dutch nationwide ATHENA cohort. Clin Infect Dis 2015; 60: 143153.

32 Lee FJ, Amin J, Carr A. Efficacy of initial antiretroviral therapy for HIV-1 infection in adults: a systematic review and meta-analysis of 114 studies with up to 144 weeks' follow-up. PLoS One 2014; 9: e97482.

\subsection{Which third agent}

\subsubsection{Recommendations}

- We recommend therapy-naïve individuals start combination ART containing atazanavir/r, darunavir/r, dolutegravir, elvitegravir/c, raltegravir or rilpivirine as the third agent (1A).

- We suggest that for therapy-naïve individuals, efavirenz is an acceptable alternative third agent (1A).

\subsubsection{Rationale}

The BHIVA guidelines for the treatment of HIV-1-infected adults with antiretroviral therapy 2008 [1] recommended efavirenz as the preferred third agent in view of significantly better virological outcomes compared with lopina$\operatorname{vir} / \mathrm{r}$ [2], subsequently confirmed in a smaller study [3]. In the following version of the guidelines, the preferred third agents were expanded to include more agents based on performance against efavirenz (atazanavir/r [4-9]; 
raltegravir [10-13]; elvitegravir/c [14]), lopinavir/r (atazanavir/r [15]; darunavir/r [16-18]) and atazanavir/r (elvitegravir/c [19]). Rilpivirine was added as an alternative third agent based on comparison with efavirenz $[15,20,21]$.

Since the latest update to guidelines was published [22], the integrase inhibitor dolutegravir and the fixeddose combination of abacavir/lamivudine/dolutegravir (Triumeq) has received licensing approval. Additionally there have also been a number of new randomised controlled trials comparing third agents for initial antiretroviral therapy. For the current guidelines, evidence for agreed treatment outcomes for each potential third agent was compared with preferred third agents from the previous guidelines [22], either directly or indirectly depending on the available evidence (Appen$\operatorname{dix} 3)$ :

- Efavirenz vs. rilpivirine [14];

- Efavirenz vs. dolutegravir [19];

- Atazanavir/r vs. darunavir/r vs. raltegravir [16];

- Raltegravir vs. dolutegravir [18];

- Darunavir/r vs. dolutegravir [17].

\subsubsection{Individual agents}

\subsubsection{Atazanavir/ritonavir}

Atazanavir/r has been compared directly in randomised controlled trials with efavirenz [7,23,24], raltegravir [16] and darunavir/r [16,25].

In ACTG 5202, although rates of virological failure were similar, there were significantly fewer cases of resistance development on atazanavir/r compared with efavirenz (OR 0.24, 95\% CI 0.14-0.41) at 138 weeks, $P<0.00001)$. Some adverse events favoured atazanavir $/ \mathrm{r}$ over efavirenz (see Appendix 3)

In ACTG 5257, the odds ratio for developing resistance at virological failure on raltegravir compared with atazanavir/r was $2.04 \quad(95 \% \quad C I \quad 0.91-4.57, \quad P=0.08)$ at 96 weeks. Although, for overall response, atazanavir/r was outperformed by raltegravir (OR 2.04, 95\% CI 1.30$3.18, P=0.002$ ) at 96 weeks in favour of raltegravir, driven mainly by significant differences in adverse event discontinuations (OR 0.07 favouring raltegravir at 96 weeks, 95\% CI $0.03-0.15, P<0.0001$ ), virological failure rates (\% total population) were similar in the two arms (OR 0.88, 95\% CI 0.64-1.21, $P=0.43$ ). Resistance development was more common on raltegravir than atazanavir/r when considering the proprortion of virological failures with resistance (OR 2.57, 95\% CI 1.08-6.08), but when considering the proportion of the total trial population developing resistance, this was not statistically significant (OR 2.04, 95\% CI 0.91-4.57).
Virological response to atazanavir/r was similar to darunavir/r in ACTG 5257 and ATADAR at 96 and 48 weeks, respectively. There were no significant differences in virological failure or resistance development rates in either study (reported only at week 24 for ATADAR). Compared to darunavir/r, individuals randomised to receive atazanavir/r in ACTG 5257 were significantly more likely to discontinue due to adverse events (OR 0.30 favouring darunavir/r, 95\% CI 0.20-0.46, $P<0.00001$ ). Although discontinuation for adverse events is a critical outcome, there was no difference in discontinuations for the specific non-critical outcomes considered (Appendix 3) and around half of discontinuations from atazanavir/r were for hyperbilirubinaemia/jaundice. On this basis it was felt that the difference between atazanavir/ $\mathrm{r}$ and its comparators was not clinically significant and that atazanavir/r should remain a preferred third agent for initial therapy. However, jaundice is a potentially distressing and stigmatising event and PLWH who do not want to start atazanavir or wish to switch from atazanavir for this reason should have this decision respected. The requirement for pharmacokinetic boosting necessitates caution with respect to drug-drug interactions.

\subsubsection{Darunavir/ritonavir}

Comparison between darunavir/ $\mathrm{r}$ and atazanavir $/ \mathrm{r}$ is described in Section 5.4.3.1. For the comparison between darunavir/r and raltegravir in the three-arm ACTG 5257 study [16], overall response was significantly higher for raltegravir (OR 1.83, 95\% CI 1.16-2.89 at 96 weeks in favour of raltegravir, $P=0.009$ ). The corresponding proportion of patients with an undetectable HIV-1 RNA at 96 weeks by intention-to-treat analysis was 88.3\% for atazanavir/r, 93.9\% for raltegravir, and 89.4\% for darunavir/r. Although there were more virological failures on darunavir/r (OR 0.69 favouring raltegravir, 95\% CI 0.510.94, $P=0.02$ ), individuals on raltegravir were more likely to develop resistance (OR 4.59, 95\% CI 1.54-13.65, $P=0.006$ ) favouring darunavir/r for percentage of the total population with resistance. There were fewer discontinuations for toxicity in the raltegravir arm (8/603 vs. $32 / 601$ in the darunavir/r arm, OR 0.24 , 95\% CI $0.11-$ 0.52); however, for the critical outcomes of grade 3 or 4 clinical or laboratory adverse events, grade 3 or 4 headache and grade 3 or 4 diarrhoea there were no significant differences. Darunavir/r was also compared with dolutegravir in the Flamingo study, and is discussed in Section 5.4.3.3. The requirement for pharmacokinetic boosting necessitates caution with respect to drug-drug interactions.

5.4.3.3 Dolutegravir

Dolutegravir has gained marketing authorisation and NHS England approval since the last guidelines update. 
There are three key randomised controlled trials that investigate dolutegravir for first-line therapy:

- SPRING-2 [18]: double-blind randomised controlled trial of tenofovir-DF/emtricitabine or abacavir/lamivudine plus raltegravir $v s$. tenofovir-DF/emtricitabine or abacavir/lamivudine plus dolutegravir.

- SINGLE [19]: double-blind randomised controlled trial trial of tenofovir-DF/emtricitabine/efavirenz (fixeddose combination) $v s$. abacavir/lamivudine plus dolutegravir.

- FLAMINGO [17]: open label randomised controlled trial trial tenofovir-DF/emtricitabine or abacavir/lamivudine plus darunavir/r vs. tenofovir-DF/emtricitabine or abacavir/lamivudine plus dolutegravir.

In SPRING-2, dolutegravir was non-inferior to raltegravir at weeks 48 and 96 [18]. When analysed by baseline viral load (participants were stratified by baseline viral load at randomisation) there was no significant difference in virological response at baseline viral load $>100,000$ copies $/ \mathrm{mL}$ at 48 weeks (OR for success on dolutegravir $=1.57,95 \%$ CI $0.83-2.97, P=0.17$ ) but by week 96 there was a significant difference favouring dolutegravir (OR for success on dolutegravir $=2.10$, 95\% CI 1.17-3.75, $P=0.01$ ). Overall virological failure rates at 48 and 96 weeks were not significantly different; and there was no significant difference for baseline viral load $<100,000$ copies/mL at 48 or 96 weeks, or for baseline viral load $>100,000$ copies $/ \mathrm{mL}$ at 48 weeks. SPRING-2 was not powered for a stratified viral load comparison. Rates of virological failure and resistance development were similar and no differences in other critical or important outcomes were demonstrated.

In SINGLE [19], abacavir/lamivudine/dolutegravir demonstrated superior virological efficacy to tenofovirDF/emtricitabine/efavirenz, driven by significantly more discontinuations due to adverse events in the tenofovir$\mathrm{DF} /$ emtricitabine/efavirenz arm (OR for discontinuation for adverse events $=0.22$ favouring abacavir/lamivudine/ dolutegravir, 95\% CI 0.11-0.45, $P<0.001)$. There was no impact of baseline viral load on respsonse rates in SINGLE.

Dolutegravir also demonstrated superior overall efficacy compared with darunavir/r in FLAMINGO (OR for success at 48 weeks $=1.08, \quad 95 \% \quad$ CI $1.01-1.17$, $P=0.03)$ [17]. There was no difference in rates of virological failure and no instance of drug resistance in either arm. No differences were detected for other critical outcomes, although for important outcomes, there were significantly more clinical serious adverse events in the dolutegravir arm (OR 2.00, 95\% CI 1.05-3.80, $P=0.03)$.
The consensus of the Writing Group was that, despite the superiority of dolutegravir in this study, the openlabel trial design and lack of difference for other critical outcomes, meant darunavir/ $\mathrm{r}$ should remain a preferred third agent.

5.4.3.4 Elvitegravir/cobicistat

Elvitegravir/c was a preferred option in the last update and remains so, based on demonstration of non-inferiority to efavirenz and atazanavir/r. In GS-102 [26], elvitegravir/c was non-inferior to efavirenz at weeks 48 and 96 in terms of virological success, virological failure and proportion of people who developed resistance; rates of NRTI and third-agent resistance development in individuals with virological failure were similar. For example, by week 96, 9/17 persons with virological failure on elvitegravir/c developed integrase resistance compared with 9/ 23 with virological failure on efavirenz. There were no differences in adverse event discontinuations or rate of grade $3 / 4$ adverse event outcomes, with data lacking for some of the non-critical safety outcomes considered by the writing group. Elvitegravir/c has the advantage of simple dosing as a fixed-dose combination with tenofovir-DF/emtricitabine; the requirement for pharmacokinetic boosting necessitates caution with respect to drugdrug interactions.

When compared with atazanavir/r in GS-103 [27], elvitegravir/c was again non-inferior with respect to virological success and virological failure rates at weeks 48 and 96. There were no cases of NRTI- or PI-resistance development at weeks 48 and 96 in the atazanavir/r arm compared with five and six cases in the elvitegravir/c arm, respectively. By week 144, there were eight cumulative cases of resistance in the elvitegravir/c arm compared with two in the atazanavir/r (both isolated M184I/ V) [28]. Discontinuations for adverse events were similar and the only important outcome that differed was a significantly higher rate of grade 3/4 laboratory events in the atazanavir/r arm (OR 0.07 favouring elvitegravir/c, 95\% CI 0.05-0.11, $P<0.0001$ at week 48). Again, as this was driven predominantly by hyperbilirubinaemia in the atazanavir/r arm, and the consensus of the writing group was that this difference was not clinically important.

Cobicistat is an inhibitor of renal tubular creatinine secretion; exposure to cobicistat results in small increases in serum creatinine and modest reductions (10-15 mL/ min) in creatinine clearance [29]. Renal toxicity with elvitegravir/cobicistat/tenofovir-DF/emtricitabine is infrequent $(0.8-2 \%)$ in patients with baseline creatinine clearance at least $70 \mathrm{~mL} / \mathrm{min}[26,27]$. The use of elvitegravir/ cobicistat/tenofovir-DF/emtricitabine is not recommended for patients with creatinine clearance less than $70 \mathrm{~mL} /$ $\min [30]$. 


\subsubsection{Raltegravir}

Raltegravir was compared to atazanavir/r, darunavir/r and dolutegravir in studies outlined in Sections 5.4.3.1, 5.4.3.2 and 5.4.3.3, respectively. In ACTG 5257 virological failure with resistance occurred in 3.0\% of study participants assigned to raltegravir (two of whom developed intermediate-level resistance to dolutegravir) and in 1.5\% or fewer of those in either boosted PI group [16]. Raltegravir was non-inferior to efavirenz in STARTMRK with similar outcomes for virological response, virological failure and resistance rates at weeks 48 [12] and 96 [11]. The study was unblinded after week 144 and at later points of follow-up (up to week 240) raltegravir demonstrated superior efficacy to efavirenz driven by more discontinuations due to adverse-events in the efavirenz arm [31]. There was a trend to more discontinuations for adverse events on efavirenz at weeks 48 and 96 (OR 0.52 and 0.51 favouring raltegravir, respectively) and by week 240 , this difference reached statistical significance. Unlike other preferred third agents for first-line use, the licensed dose of raltegravir is taken twice daily.

5.4.3.6 Rilpivirine

Rilpivirine has been compared directly with efavirenz in three large randomised controlled trial trials: ECHO [32], THRIVE (both double-blind, placebo-controlled, multi-pill studies) [15] and StAR (open-label single-pill fixed-dose combination comparison of tenofovir-DF/emtricitabine/ efavirenz and tenofovir-DF/emtricitabine/rilpivirine) [14]. There were no differences in overall virological success at weeks 48 or 96 in the three studies. However, there were significant differences in drug resistance and virological failure, both in favour of efavirenz. For critical safety outcomes there was a difference in the proportion discontinuing for adverse events in favour of rilpivirine. Pooled analyses by the investigators of the two randomised controlled trial trials showed the risk of virological failure with rilpivirine was highest in participants with a baseline viral load $>100,000$ copies/mL [15] and when analysis is restricted to individuals with a baseline viral load less than 100,000 copies/mL, virological response to rilpivirine was non-inferior to efavirenz. A 96-week analysis of the same studies, restricted to individuals with baseline viral load less than 100,000 copies/mL, demonstrated virological response rates of $84 \%$ on rilpivirine and $80 \%$ on efavirenz (95\% CI -1.7 to $9.7 \%$ ) and virological failure rates of $8 \%$ and $6 \%$, respectively $(P=0.46)$ [32].

Based on the superior virological success on rilpivirine compared to efavirenz when used within its licensed indication (i.e. at baseline viral load less than 100,000 copies/ $\mathrm{mL}$ ) we believe it should be moved from alternative to preferred third agent status. It is important to note that there are very few data regarding the administration of rilpivirine with an abacavir/lamivudine NRTI backbone. In addition rilpivirine must be administered with food (Appendix 4) and has significant interactions with acidreducing agents; proton pump inhibitors are contraindicated.

5.4.3.7 Efavirenz

Efavirenz has long been a preferred agent for initial HIV therapy and has been a 'gold standard' comparator for several clinical trials investigating first-line treatment. However, dolutegravir demonstrated superiority to efavirenz in SINGLE [19], as has rilpivirine in subgroup analyses [33], and raltegravir with longer-term follow-up of STARTMRK [31]. The difference between efavirenz and comparators is driven by a higher rate of discontinuation for adverse events on efavirenz-based regimens, mainly due to its potential for significant central nervous system (CNS) toxicity. Since the last update of the guidelines, a meta-analysis of ACTG studies demonstrated a higher risk of suicidality in those randomised to efavirenz-containing regimens [34]. Individuals with significant past or current mental health issues may be excluded from clinical trial populations so the ACTG analysis could potentially underestimate the impact of efavirenz in higher-risk PLWH. Although, subsequently, an observational cohort study did not demonstrate an association between efavirenz use and suicidality [35] this was a non-randomised, retrospective analysis. Individuals with a history of psychiatric disorders appear to be at a greater risk of serious psychiatric adverse events [36] and for PLWH with a current or previous history of psychiatric disorders, including depression, anxiety and suicidal ideation, caution should be exercised in prescribing efavirenz and strong consideration given to using an alternative third agent. Secondarily, although not considered an important outcome for the purpose of these guidelines, efavirenz is associated with an adverse impact on lipids compared to newer agents. Since there are several effective and welltolerated alternative third agent options available, it is the view of the writing group that efavirenz should be downgraded from preferred to alternative option for initial therapy. The writing group recognises that the majority of individuals who start efavirenz-based therapy tolerate it reasonably well, so it remains a reasonable alternative. For patients stable on efavirenz-based ART, we recommend a review of tolerability, including sleep and mood, at all visits.

5.4.3.8 Previous alternative third agents

Unlike previous versions of the guidelines, lopinavir/r, fosamprenavir/r and nevirapine are no longer listed as alternative options. Efavirenz was superior to lopinavir/r for critical virological outcomes in ACTG 5142 [2]; lopinavir/r and fosamprenavir/r perform similarly [37] and 
are associated with higher rates of gastrointestinal and metabolic toxicities than preferred PI/r options [38]. Lopinavir/r may be an important option for some individuals such as those with PI resistance mutations and a contraindication for darunavir/r. Nevirapine was non-inferior to atazanavir/r for critical virological outcomes in ARTEN [39] but with the availability of alternative NNRTIs and alternative classes, we feel that the small risk of serious hepatic or cutaneous toxicity is no longer acceptable. Individuals who are stable on nevirapine should, however, be reassured as to the long-term efficacy and safety of this option.

\subsubsection{Cobicistat}

Cobicistat is an inhibitor of CYP3A isozymes [40] and, similarly to ritonavir, is used as a pharmacokinetic enhancer of other antiretroviral drugs. It is a component in the fixed-dose combination pill Stribild (elvitegravir/ cobicistat/emtricitabine/tenofovir-DF). Cobicistat as a single drug has also been licensed as a pharmacokinetic enhancer of atazanavir and darunavir.

The efficacy and safety of cobicistat-boosted atazanavir has been evaluated in comparison with ritonavir-boosted atazanavir, in a Phase 3, non-inferiority, randomised, double-blind study in treatment-naïve individuals [41,42]. Atazanavir with cobicistat was shown to be non-inferior to atazanavir with ritonavir in terms of viral suppression. Discontinuation rates due to adverse events were similar, and no major tolerability issues were reported.

The evidence supporting the use of cobicistat to boost darunavir is more limited. Cobicistat enhances darunavir plasma levels to a similar degree as ritonavir, with a similar pharmacokinetic profile [43] and in a single-arm study had acceptable efficacy and safety profiles [44].

Cobicistat affects tubular secretion of creatinine, by inhibiting renal cation transporters. This results in small increases in serum creatinine and decreases in estimated creatinine clearance. However, it does not cause impairment of renal function as actual glomerular filtration rate is not affected [45].

\subsubsection{Summary}

When selecting a third agent from either the preferred or alternative options, factors such as potential side-effects, dosing requirements, dosing convenience, individual preference, co-morbidities, drug interactions and cost should be considered.

In summary, efavirenz should no longer remain a preferred third agent and should now be considered an alternative. Because of similar critical treatment outcomes, atazanavir/r, darunavir/r, dolutegravir elvitegravir/c, raltegravir and rilpivirine are all recommended as preferred third agents (with the caveat that rilpivirine is only recommended within its licence in individuals with a baseline viral load less than 100,000 copies/mL).

\subsubsection{References}

1 Gazzard BG, Anderson J, Babiker A et al. British HIV Association Guidelines for the treatment of HIV-1-infected adults with antiretroviral therapy 2008. HIV Med 2008; 9: 563-608.

2 Riddler SA, Haubrich R, DiRienzo AG et al. Class-sparing regimens for initial treatment of HIV-1 infection. $N$ Engl $J$ Med 2008; 358: 2095-2106.

3 Sierra-Madero J, Villasis-Keever A, Mendez P et al. Prospective, randomized, open label trial of efavirenz vs lopinavir/ritonavir in HIV+ treatment-naive subjects with CD4 $+<200$ cell/mm3 in Mexico. J Acquir Immune Defic Syndr 2010; 53: 582-588.

4 McComsey GA, Kitch D, Daar ES et al. Bone mineral density and fractures in antiretroviral-naive persons randomized to receive abacavir-lamivudine or tenofovir disoproxil fumarate-emtricitabine along with efavirenz or atazanavirritonavir: Aids Clinical Trials Group A5224s, a substudy of ACTG A5202. J Infect Dis 2011; 203: 1791-1801.

5 McComsey GA, Kitch D, Sax PE et al. Peripheral and central fat changes in subjects randomized to abacavir-lamivudine or tenofovir-emtricitabine with atazanavir-ritonavir or efavirenz: ACTG Study A5224s. Clin Infect Dis 2011; 53: 185-196.

6 Puls RL, Srasuebkul P, Petoumenos K et al. Efavirenz versus boosted atazanavir or zidovudine and abacavir in antiretroviral treatment-naive, HIV-infected subjects: week 48 data from the Altair study. Clin Infect Dis 2010; 51: 855-864.

7 Sax PE, Tierney C, Collier AC et al. Abacavir/lamivudine versus tenofovir DF/emtricitabine as part of combination regimens for initial treatment of HIV: final results. J Infect Dis 2011; 204: 1191-1201.

8 Sax PE, Tierney C, Collier AC et al. Abacavir-lamivudine versus tenofovir-emtricitabine for initial HIV-1 therapy. $N$ Engl J Med 2009; 361: 2230-2240.

9 Winston A, Duncombe C, Li PC et al. Does choice of combination antiretroviral therapy (cART) alter changes in cerebral function testing after 48 weeks in treatment-naive, HIV-1-infected individuals commencing cART? A randomized, controlled study. Clin Infect Dis 2010; 50: 920929.

10 Gotuzzo E, Markowitz M, Ratanasuwan W et al. Sustained efficacy and safety of raltegravir after 5 years of combination antiretroviral therapy as initial treatment of HIV-1 infection: final results of a randomized, controlled, phase II study (Protocol 004). J Acquir Immune Defic Syndr 2012; 61: 73-77. 
11 Lennox JL, Dejesus E, Berger DS et al. Raltegravir versus efavirenz regimens in treatment-naive HIV-1-infected patients: 96-week efficacy, durability, subgroup, safety, and metabolic analyses. J Acquir Immune Defic Syndr 2010; 55: 39-48.

12 Lennox JL, DeJesus E, Lazzarin A et al. Safety and efficacy of raltegravir-based versus efavirenz-based combination therapy in treatment-naive patients with HIV-1 infection: a multicentre, double-blind randomised controlled trial. Lancet 2009; 374: 796-806.

13 Markowitz M, Nguyen BY, Gotuzzo E et al. Sustained antiretroviral effect of raltegravir after 96 weeks of combination therapy in treatment-naive patients with HIV-1 infection. J Acquir Immune Defic Syndr 2009; 52: 350-356.

14 Porter DP, Kulkarni R, Fralich T et al. 96-week resistance analyses of the STaR study: rilpivirine/emtricitabine/ tenofovir DF versus efavirenz/emtricitabine/tenofovir DF in antiretroviral-naive, HIV-1-infected subjects. HIV Clin Trials 2015; 16: 30-38.

15 Nelson MR, Elion RA, Cohen CJ et al. Rilpivirine versus efavirenz in HIV-1-infected subjects receiving emtricitabine/ tenofovir DF: pooled 96-week data from ECHO and THRIVE Studies. HIV Clin Trials 2013; 14: 81-91.

16 Lennox JL, Landovitz RJ, Ribaudo HJ et al. Efficacy and tolerability of 3 nonnucleoside reverse transcriptase inhibitor-sparing antiretroviral regimens for treatment-naive volunteers infected with HIV-1: a randomized, controlled equivalence trial. Ann Intern Med 2014; 161: 461-471.

17 Molina JM, Clotet B, van Lunzen J et al. Once-daily dolutegravir is superior to once-daily darunavir/ritonavir in treatment-naive HIV-1-positive individuals: 96 week results from FLAMINGO. J Int AIDS Soc 2014; 17: 19490.

18 Raffi F, Jaeger H, Quiros-Roldan E et al. Once-daily dolutegravir versus twice-daily raltegravir in antiretroviralnaive adults with HIV-1 infection (SPRING-2 study): 96 week results from a randomised, double-blind, noninferiority trial. Lancet Infect Dis 2013; 13: 927-935.

19 Walmsley SL, Antela A, Clumeck N et al. Dolutegravir plus abacavir-lamivudine for the treatment of HIV-1 infection. $N$ Engl J Med 2013; 369: 1807-1818.

20 Cohen CJ, Andrade-Villanueva J, Clotet B et al. Rilpivirine versus efavirenz with two background nucleoside or nucleotide reverse transcriptase inhibitors in treatmentnaive adults infected with HIV-1 (THRIVE): a phase 3, randomised, non-inferiority trial. Lancet 2011; 378: 229237.

21 Molina JM, Cahn P, Grinsztejn B et al. Rilpivirine versus efavirenz with tenofovir and emtricitabine in treatmentnaive adults infected with HIV-1 (ECHO): a phase 3 randomised double-blind active-controlled trial. Lancet 2011; 378: 238-246.
22 Writing Group, Williams I, Churchill D et al. British HIV Association guidelines for the treatment of HIV-1-positive adults with antiretroviral therapy 2012 (Updated November 2013). HIV Med 2014; 15 (Suppl 1): 1-85.

23 Daar ES, Tierney C, Fischl MA et al. Atazanavir plus ritonavir or efavirenz as part of a 3-drug regimen for initial treatment of HIV-1. Ann Intern Med 2011; 154: 445-456.

24 Josephson F, Andersson MC, Flamholc L et al. The relation between treatment outcome and efavirenz, atazanavir or lopinavir exposure in the NORTHIV trial of treatment-naive HIV-1 infected patients. Eur J Clin Pharmacol 2010; 66: 349-357.

25 Aberg JA, Tebas P, Overton ET et al. Metabolic effects of darunavir/ritonavir versus atazanavir/ritonavir in treatmentnaive, HIV type 1-infected subjects over 48 weeks. AIDS Res Hum Retroviruses 2012; 28: 1184-1195.

26 Zolopa A, Sax PE, DeJesus E et al. A randomized doubleblind comparison of coformulated elvitegravir/cobicistat/ emtricitabine/tenofovir disoproxil fumarate versus efavirenz/emtricitabine/tenofovir disoproxil fumarate for initial treatment of HIV-1 infection: analysis of week 96 results. J Acquir Immune Defic Syndr 2013; 63: 96-100.

27 Rockstroh JK, DeJesus E, Henry K et al. A randomized, double-blind comparison of coformulated elvitegravir/ cobicistat/emtricitabine/tenofovir DF vs ritonavir-boosted atazanavir plus coformulated emtricitabine and tenofovir DF for initial treatment of HIV-1 infection: analysis of week 96 results. J Acquir Immune Defic Syndr 2013; 62: 483-486.

28 Kulkarni R, Abram ME, McColl DJ et al. Week 144 resistance analysis of elvitegravir/cobicistat/emtricitabine/ tenofovir DF versus atazanavir+ritonavir+emtricitabine/ tenofovir DF in antiretroviral-naive patients. HIV Clin Trials 2014; 15: 218-230.

29 Yombi JC, Pozniak A, Boffito M et al. Antiretrovirals and the kidney in current clinical practice: renal pharmacokinetics, alterations of renal function and renal toxicity. AIDS 2014; 28: 621-632.

30 Electronic Medicines Compendium. Stribild Summary of Product Characteristics, 23 June 2015. Available at: www.medicines.org.uk/emc/medicine/27810 (accessed September 2015).

31 Rockstroh JK, DeJesus E, Lennox JL et al. Durable efficacy and safety of raltegravir versus efavirenz when combined with tenofovir/emtricitabine in treatment-naive HIV-1infected patients: final 5-year results from STARTMRK. $J$ Acquir Immune Defic Syndr 2013; 63: 77-85.

32 Molina JM, Clumeck N, Orkin C et al. Week 96 analysis of rilpivirine or efavirenz in HIV-1-infected patients with baseline viral load $<=100000$ copies $/ \mathrm{mL}$ in the pooled ECHO and THRIVE phase 3, randomized, double-blind trials. HIV Med 2014; 15: 57-62. 
33 Behrens G, Rijnders B, Nelson M et al. Rilpivirine versus efavirenz with emtricitabine/tenofovir disoproxil fumarate in treatment-naive HIV-1-infected patients with HIV-1 RNA $</=100,000$ copies/mL: week 96 pooled ECHO/THRIVE subanalysis. AIDS Patient Care STDS 2014; 28: 168-175.

34 Mollan KR, Smurzynski M, Eron JJ et al. Association between efavirenz as initial therapy for HIV-1 infection and increased risk for suicidal ideation or attempted or completed suicide: an analysis of trial data. Ann Intern Med 2014; 161: 1-10.

35 Smith C, Ryom L, Monforte A et al. Lack of association between use of efavirenz and death from suicide: evidence from the D:A: D study. J Int AIDS Soc 2014; 17: 19512.

36 Electronic Medicines Compendium. Efavirenz Summary of Product Characteristics, 27 May 2014. Available at: www.medicines.org.uk/emc/medicine (accessed August 2015).

37 Eron J Jr, Yeni P, Gathe J Jr et al. The KLEAN study of fosamprenavir-ritonavir versus lopinavir-ritonavir, each in combination with abacavir-lamivudine, for initial treatment of HIV infection over 48 weeks: a randomised noninferiority trial. Lancet 2006; 368: 476-482.

38 Hill A, Balkin A. Risk factors for gastrointestinal adverse events in HIV treated and untreated patients. AIDS Rev 2009; 11: 30-38.

39 Soriano V, Arasteh K, Migrone H et al. Nevirapine versus atazanavir/ritonavir, each combined with tenofovir disoproxil fumarate/emtricitabine, in antiretroviral-naive HIV-1 patients: the ARTEN Trial. Antivir Ther 2011; 16: 339-348.

40 Mathias AA, German P, Murray BP et al. Pharmacokinetics and pharmacodynamics of GS-9350: a novel pharmacokinetic enhancer without anti-HIV activity. Clin Pharmacol Ther 2010; 87: 322-329.

41 Gallant JE, Koenig E, Andrade-Villanueva J et al. Cobicistat versus ritonavir as a pharmacoenhancer of atazanavir plus emtricitabine/tenofovir disoproxil fumarate in treatmentnaive HIV type 1-infected patients: week 48 results. J Infect Dis 2013; 208: 32-39.

42 Gallant JE, Koenig E, Andrade-Villanueva JF et al. Brief Report: Cobicistat Compared With Ritonavir as a Pharmacoenhancer for Atazanavir in Combination With Emtricitabine/Tenofovir Disoproxil Fumarate: Week 144 Results. J Acquir Immune Defic Syndr 2015; 69: 338-340.

43 Mathias A, Liu HC, Warren D, Sekar V. Relative bioavailability and pharmacokinetics of darunavir when boosted with the pharmacoenhancer GS-9350 versus ritonavir. 11th International Workshop on Clinical Pharmacology of HIV Therapy. April 2010. Sorrento, Italy [Abstract 28].

44 Tashima K, Crofoot G, Tomaka FL et al. Phase IIIb, openlabel single-arm trial of darunavir/cobicistat (DRV/COBI):
Week 48 subgroup analysis of HIV-1-infected treatmentnave adults. J Int AIDS Soc 2014; 17: 19772.

45 German P, Liu HC, Szwarcberg J et al. Effect of cobicistat on glomerular filtration rate in subjects with normal and impaired renal function. J Acquir Immune Defic Syndr 2012; 61: 32-40.

\subsection{Novel antiretroviral therapy strategies}

\subsubsection{Recommendation}

- We recommend against the use of PI monotherapy as initial therapy for treatment-naïve patients (1C).

\subsubsection{Rationale}

Data on use of PI monotherapy as initial ART are limited. In one randomised controlled trial comparing lopinavir/r $v s$. lopinavir/r plus zidovudine and lamivudine, the use of PI monotherapy as initial ART was associated with lower rates of virological suppression at 48 weeks and with the emergence of PI mutations [1]. There were no significant differences in tolerability. For this reason, PI monotherapy is not recommended as initial ART. However, as with other novel strategies there may be specific circumstances where a rationale for its use may be made.

\subsubsection{Reference}

1 Delfraissy JF, Flandre P, Delaugerre C et al. Lopinavir/ ritonavir monotherapy or plus zidovudine and lamivudine in antiretroviral-naive HIV-infected patients. AIDS 2008; 22: 385-393.

\subsubsection{Recommendation}

- We suggest the use of darunavir/r-based dual ART regimen with raltegravir in treatment-naïve patients with CD4 count $>200$ cells $/ \mu \mathrm{L}$ and viral load $<100,000$ copies/mL where there is need to avoid abacavir and tenofovir-DF (2A).

- We recommend against the use of PI-based dual ART with a single NNRTI, NRTI or CCR5 receptor antagonist for treatment-naïve patients (1B).

5.5.2.1 Rationale

A number of studies have assessed the use of PI-based dual ART as initial therapy in treatment-naïve patients.

The combination of an NNRTI with a PI/r has been shown to have similar virological efficacy compared with triple-combination regimens in one study [1]. There were no significant differences in time to either virological or regimen failure with a combination of lopinavir/ $\mathrm{r}$ and efavirenz compared with either two NRTIs and efavirenz or two NRTIs and lopinavir/r although the NRTI-sparing arm underperformed in individuals with high baseline viral load (greater than 100,000 copies/mL). There was, 
however, an increased rate of drug resistance in the NRTI-sparing arm, with the emergence of more NNRTIassociated resistance mutations than the comparator arms. An increased rate of grade 3/4 toxicities was observed, predominantly low-density lipoprotein cholesterol and triglyceride elevations.

Two randomised controlled trial trials have evaluated a dual-therapy regimen containing one NRTI with a PI/r compared to standard therapy of a PI/r and two NNRTIs. The GARDEL study demonstrated non-inferiority of the dual regimen of lopinavir/r plus lamivudine compared to lopinavir/r, lamivudine or emtricitabine plus a third NRTI in virological efficacy at 48 weeks [2] irrespective of baseline viral load. A post hoc analysis also showed no difference in virological efficacy with respect to the choice of dual NRTI backbone and pre-treatment CD4 cell count $(<200$ cells $/ \mu \mathrm{L})$ [3]. A study comparing tenofovirDF and lopinavir/r to two NRTIs and lopinavir/r failed to demonstrate non-inferiority of the dual-therapy arm compared with a standard triple-therapy combination but numbers were small and the response rates were numerically similar at 51\% and 53\%, respectively [4]. As lopina$\mathrm{vir} / \mathrm{r}$ is no longer among the first-recommended PIs, the dual combination with lamivudine, as used in GARDEL study, has not been considered as an alternative first-line regimen.

The efficacy of dual therapy with the CCR5-receptor antagonist maraviroc in combination with a $\mathrm{PI} / \mathrm{r}$ has been assessed in a number of studies [5-8] but only one [8] was powered to demonstrate non-inferiority. This study compared maraviroc/darunavir/ritonavir to tenofovir DF/ emtricitabine/darunavir/r, and showed lower virological efficacy of the dual therapy arm at 48 weeks.

The efficacy of the raltegravir plus a $\mathrm{PI} / \mathrm{r}$ has been compared with standard triple therapy in several studies [9-13] The NEAT-001/ANRS-143 [10] demonstrated noninferiority of raltegravir compared to tenofovir-DF/ emtricitabine when combined with darunavir/r at 96 weeks. However, the dual-therapy arm was associated with higher rates of virological failure and with treatment-emergent integrase resistance (5/28 patients) in those with baseline CD 4 cell count $<200$ cells $/ \mu \mathrm{L}$ or viral load $>100,000$ copies/mL. Similarly, a single-arm study investigating raltegravir in combination also with darunavir/r, showed increased risk of virological failure with emergent integrase resistance with baseline viral load $>100,000$ copies/mL compared with those with a baseline viral load $<100,000$ copies/mL [13]. There was no difference in safety between both arms.

The PROGRESS study demonstrated similar virological efficacy of raltegravir plus lopinavir/r compared to tenofovir-DF/emtricitabine and lopinavir/r, although the study was relatively small $[11,12]$. Overall there is evidence to support the use of a $\mathrm{PI} / \mathrm{r}$, preferably darunavir/r plus raltegravir, in selected patient populations.

The use of raltegravir with unboosted atazanavir is not recommended due to development of integrase resistance in $4 / 6$ of those who met the criteria for resistance testing [9].

\subsubsection{References}

1 Riddler SA, Haubrich R, DiRienzo AG et al. Class-sparing regimens for initial treatment of HIV-1 infection. $N$ Engl $J$ Med 2008; 358: 2095-2106.

2 Cahn P, Andrade-Villanueva J, Arribas JR et al. Dual therapy with lopinavir and ritonavir plus lamivudine versus triple therapy with lopinavir and ritonavir plus two nucleoside reverse transcriptase inhibitors in antiretroviraltherapy-naive adults with HIV-1 infection: 48 week results of the randomised, open label, non-inferiority GARDEL trial. Lancet Infect Dis 2014; 14: 572-580.

3 Rolon MJ, Figueroa MI, Patterson P et al. Dual therapy efficacy in key subgorups of treatment-naïve patients in the GARDEL study. 20th International AIDS Conference. July 2014. Melbourne, Australia [Abstract WEPE080].

4 Pinola M, Lazzarin A, Antinori A et al. Lopinavir/ritonavir + tenofovir dual therapy versus lopinavir/ritonavir-based triple therapy in HIV-infected antiretroviral naïve subjects: the Kalead Study. J Antivir Antiretrovir 2010; 2: 056-062.

5 Mills A, Mildvan D, Podzamczer D et al. Maraviroc oncedaily nucleoside analog-sparing regimen in treatment-naive patients: randomized, open-label pilot study. J Acquir Immune Defic Syndr 2013; 62: 164-170.

6 Nozza S, Galli L, Chiappetta S et al. Maraviroc $150 \mathrm{mg}$ QD plus lopinavir/ritonavir, a NRTI-sparing regimen for HIVinfected naivve patients: 48-weeks final results. J Int AIDS Soc 2012; 15 (Suppl 4): 18232.

7 Portsmouth S, Craig C, Mills A et al. 48-week results of once-daily maraviroc (MVC) $150 \mathrm{mg}$ in combination with ritonavir-boosted atazanavir (ATV/r) compared to emtricitabine/tenofovir (FTC/TDF) + ATV/r in treatmentnaïve patients infected with R5 HIV-1 (Study A4001078). 6th IAS Conference on HIV Pathogenesis, Treatment and Prevention. July 2011. Rome, Italy [Abstract TUAB0103].

8 Stellbrink HJ, Pulik P, Szlavik J. Maraviroc (MVC) once daily with darunavir/ritonavir (DRV/r) compared to tenofovir/emtricitabine (TDF/FTC) with DRV/r: 48-week results from MODERN (Study A4001095). International AIDS Conference. July 2014. Melbourne, Australia [Abstract TUAB0101].

9 Kozal MJ, Lupo S, DeJesus E et al. A nucleoside- and ritonavir-sparing regimen containing atazanavir plus raltegravir in antiretroviral treatment-naive HIV-infected 
patients: SPARTAN study results. HIV Clin Trials 2012; 13: 119-130.

10 Raffi F, Babiker AG, Richert L et al. Ritonavir-boosted darunavir combined with raltegravir or tenofoviremtricitabine in antiretroviral-naive adults infected with HIV-1: 96 week results from the NEAT001/ANRS143 randomised non-inferiority trial. Lancet 2014; 384: 19421951.

11 Reynes J, Lawal A, Pulido F et al. Examination of noninferiority, safety, and tolerability of lopinavir/ritonavir and raltegravir compared with lopinavir/ritonavir and tenofovir/ emtricitabine in antiretroviral-naive subjects: the progress study, 48-week results. HIV Clin Trials 2011; 12: 255-267.

12 Reynes J, Trinh R, Pulido F et al. Lopinavir/ritonavir combined with raltegravir or tenofovir/emtricitabine in antiretroviral-naive subjects: 96-week results of the PROGRESS study. AIDS Res Hum Retroviruses 2013; 29: 256-265.

13 Taiwo B, Zheng L, Gallien S et al. Efficacy of a nucleosidesparing regimen of darunavir/ritonavir plus raltegravir in treatment-naive HIV-1-infected patients (ACTG A5262). AIDS 2011; 25: 2113-2122. 


\subsection{Supporting individuals on therapy}

\subsection{Adherence}

\subsubsection{Interventions to increase adherence to treatment}

6.1.1.1 Recommendations

- We recommend adherence and potential barriers to it are assessed and discussed with PLWH whenever ART is discussed, prescribed or dispensed (GPP).

- We recommend adherence support should address both perceptual barriers (e.g. beliefs and preferences) and/or practical barriers (e.g. limitations in capacity and resources) to adherence (GPP).

- Individuals experiencing difficulties with adherence should be offered additional support from staff within the MDT who have experience in adherence support and/or from organisations offering peer support (GPP).

6.1.1.2 Auditable outcomes

- Record in medical notes discussion and assessment of adherence and potential barriers, both before starting a new ART regimen and while on ART.

- Record in medical notes the provision or offer of adherence support.

6.1.1.3 Rationale

Low adherence to ART is associated with drug resistance, progression to AIDS [1] and death [2-4]. Given the multiple adverse consequences of treatment failure (risk of disease progression, increase in complexity and costs of treatment, and risk of HIV transmission) engaging PLWH in treatment decisions and the monitoring and support of adherence are of paramount importance [5] (see Section 3.0). Investing time to prepare an individual for starting ART is important and utilising members of the MDT who have experience in adherence support, such as specialist nurses and pharmacists, should be considered for all individuals starting ART, reporting adherence concerns or who have experienced virological failure.

Non-adherence is best understood as a variable behaviour with intentional and unintentional causes. Most people taking medication are non-adherent some of the time. Unintentional non-adherence is linked to limitations in capacity or resources that reduce the ability to adhere to the treatment as intended. Intentional non-adherence is the product of a decision informed by beliefs, emotions and preferences [6].

BHIVA recommendations on the monitoring of adherence to ART are available (www.bhiva.org/monitoring- guidelines.aspx). The National Institute for Health and Care Excellence (NICE) has published detailed guidance on the assessment and support of adherence to medication in chronic diseases; key recommendations for adherence support are shown in Box 6.1 [7].

For PLWH it may help to normalise ambivalence and conversations about suboptimal adherence by reassuring the person that many others have doubts and concerns about ART. A person's motivation to start and continue medication is influenced by their judgement of their personal need for medication (necessity beliefs), relative to their concerns about potential adverse effects. Delayed uptake and non-adherence are associated with doubts about personal need for ART and concerns about taking it $[8,9]$. PLWH may not raise adherence concerns so should be encouraged to do so, and provided with feedback, at each visit.

Interventions to support adherence should be tailored to address specific relevant perceptual and practical barriers. A three-step 'perceptions and practicalities approach' [9] may be helpful:

- Identify and address any doubts about personal need for ART;

- Identify and address specific concerns about taking ART;

- Identify and address practical barriers to adherence.

Strategies to improve adherence should be tailored to the patient's needs, and can include the following.

- Elicit understanding of, and educate on, HIV and adherence in a way that is appropriate for the patient [10-12]. Similarly, review clinical outcomes with the patient in a way that is understandable

- Provide a rationale for treatment that is tailored to the individual $[6,13]$.

- Assess misinformation about adherence $[14,15]$.

- Clinicians should not contest miraculous healing beliefs $[14,16]$ but rather offer the compatible belief that medication and God can work together to bring health.

- Limit ART complexity and explore patient's lifestyle and work hours to select the most appropriate regimen [17].

- Many patients find it difficult to remember to take medication on time [17]. Suggestions such as using a phone or watch alarm [18], or linking an established 
Box 6.1 Summary of NICE guidance on adherence support [7]

\section{Assessment}

Recognise that non-adherence is common and that most patients are non-adherent sometimes. Routinely assess adherence in a non-judgemental way whenever you prescribe, dispense and review medicines. The purpose of assessing adherence is not to monitor patients but rather to find out whether patients need more information and support.

Make it easier for them to report non-adherence by:

- Asking the question in a way that does not apportion blame

explaining why you are asking the question

mentioning a specific time period such as 'in the past week'

$\mathrm{O}$ asking about medicine-taking behaviours such as reducing the dose, stopping and starting medicines.

- If a patient is not taking their medicines, discuss with them whether this is because of beliefs and concerns or problems about the medicines (intentional non-adherence) or because of practical problems (unintentional nonadherence).

- Find out what form of support the patient would prefer to increase their adherence to medicines.

\section{Intervention}

Patients may need support to help them make the most effective use of their medicines (e.g. further information and discussion, or practical changes to the type of medicine or the regimen). Any interventions should address the concerns and needs of individual patients. Tailor any intervention to increase adherence to the specific difficulties with adherence the patient is experiencing.

Address any beliefs and concerns that patients have that result in reduced adherence.

Interventions might include:

- Suggesting that patients record their medicine taking

- Encouraging patients to monitor their condition

- Simplifying the dosing regimen

- Using alternative packaging for the medicine

- Using a multi-compartment medicines system.

Side effects can be a problem for some patients. If this is the case you should:

- Discuss how the patient would like to deal with side effects

- Discuss the benefits, side effects and long term effects with the patient to allow them to make an informed choice

- Consider adjusting the dosage

- Consider switching to another medicine with a different risk of side effects

- Consider what other strategies might be used (for example, timing of medicines). 
daily behaviour with taking medication - for example, taking ART with a morning cup of tea may help.

- Suggest techniques to make swallowing ART easier, such as taking it with yoghurt to mask the taste.

- Suggest techniques to limit the risk of involuntary disclosure. HIV-related stigma can compromise adherence to ART $[17,19]$, and not taking medication in front of others can be protective $[14,20]$. Clinicians can offer alternative reasons as to why someone might need to take medication at a particular time to decrease concern about disclosure (e.g. taking birth control/migraine/blood pressure medication).

\subsubsection{References}

1 Bangsberg DR, Perry S, Charlebois ED et al. Non-adherence to highly active antiretroviral therapy predicts progression to AIDS. AIDS 2001; 15: 1181-1183.

2 Garcia de Olalla P, Knobel H, Carmona A et al. Impact of adherence and highly active antiretroviral therapy on survival in HIV-infected patients. J Acquir Immune Defic Syndr 2002; 30: 105-110.

3 Hogg RS, Heath K, Bangsberg D et al. Intermittent use of triple-combination therapy is predictive of mortality at baseline and after 1 year of follow-up. AIDS 2002; 16: 1051-1058.

4 Lima VD, Geller J, Bangsberg DR et al. The effect of adherence on the association between depressive symptoms and mortality among HIV-infected individuals first initiating HAART. AIDS 2007; 21: 1175-1183.

5 Bangsberg DR. A paradigm shift to prevent HIV drug resistance. PLoS Med 2008; 5: e111.

6 Horne R, Weinman J, Barber N et al. Concordance, adherence and compliance in medicine taking: a conceptual map and research priorities. London, National Institute for Health Research (NIHR) Service Delivery and Organisation (SD0) Programme; 2006. Available at: www.netscc.ac.uk/ hsdr/files/project/SDO_FR_08-1412-076_V01.pdf (accessed August 2015).

7 National Collaborating Centre for Primary Care. Medicines adherence: Involving patients in decisions about prescribed medicines and supporting adherence. NICE Clinical Guideline 76 (CG76). 2009. Available at: http:// guidance.nice.org.uk/CG76 (accessed August 2015).

8 Gonzalez JS, Penedo FJ, Llabre MM et al. Physical symptoms, beliefs about medications, negative mood, and long-term HIV medication adherence. Ann Behav Med 2007; 34: 46-55.

9 Horne R, Cooper V, Gellaitry G et al. Patients' perceptions of highly active antiretroviral therapy in relation to treatment uptake and adherence: the utility of the necessityconcerns framework. J Acquir Immune Defic Syndr 2007; 45: 334-341.
10 Bailey SC, Oramasionwu CU, Wolf MS. Rethinking adherence: a health literacy-informed model of medication self-management. J Health Commun 2013; 18: 20-30.

11 Kalichman SC, Pope H, White D et al. The association between health literacy and HIV treatment adherence: further evidence from objectively measured medication adherence. J Int Assoc Phys AIDS Care 2008; 7: 317-323.

12 Wawrzyniak AJ, Ownby RL, McCoy K, Waldrop-Valverde D. Health literacy: impact on the health of HIV-infected individuals. Current HIV/AIDS Rep 2013; 10: 295-304.

13 Horne R, Chapman S, Parham R et al. Understanding patients' adherence-related beliefs about medicines prescribed for long-term conditions: a meta-analytic review of the necessity-concerns framework. PLoS ONE 2013; 8: e80633.

14 Poliquin E, Anderson J, Spiers J, Smith JA, Horne R. What are the barriers to antiretroviral adherence in people from UK Black African and Black Caribbean communities? A qualitative study HIV Med 2013; 14: 63.

15 Simoni J, Amico K, Pearson C, Malow R. Strategies for promoting adherence to antiretroviral therapy: a review of the literature. Curr Infect Dis Rep 2008; 10: 515-521.

16 Stevenson J, Browne D, Otoro I, Duffy A. The impact of faith-based 'healing' and 'cure' claims on Africans living with HIV in the UK. HIV Med 2013; 14: 15.

17 Langebeek N, Gisolf EH, Reiss P et al. Predictors and correlates of adherence to combination antiretroviral therapy (ART) for chronic HIV infection: a meta-analysis. BMC Med 2014; 12: 142.

18 Bartlett JA. Addressing the challenges of adherence. $J$ Acquir Immune Defic Syndr 2002; 29 (Suppl 1): S2-10.

19 Katz IT, Ryu AE, Onuegbu AG et al. Impact of HIV-related stigma on treatment adherence: systematic review and meta-synthesis. J Int AIDS Soc 2013; 16: 18640.

20 Stutterheim SE, Bos AE, Shiripinda I et al. HIV-related stigma in African and Afro-Caribbean communities in the Netherlands: manifestations, consequences and coping. Psychol Health 2012; 27: 395-411.

6.1.2 Should the choice of first-line antiretroviral therapy combination be affected by risk of non-adherence? 6.1.2.1 Recommendation

- In individuals where there is clinical concern that doses may be missed intermittently, there is insufficient evidence to recommend a PI/r over NNRTI- or INI-based regimens. However, where there is a risk of frequent prolonged treatment interruptions, $\mathrm{PI} / \mathrm{r}$-based regimens may be associated with less frequent selection for drug resistance (2C).

6.1.2.2 Rationale

Clinicians are poor at both predicting future adherence to ART in naïve subjects [1] and at detecting non-adherence 
during ART [2,3]. However, in a case where a clinician or HIV-positive individual has concerns about future adherence, should this influence the choice of first-line therapy?

The consequences of low adherence depend on drug pharmacokinetics, potency, fitness of resistant strains and genetic barrier to resistance [4]. Hence, both the level and pattern of non-adherence must be considered.

Large randomised controlled trials of first-line therapy may not be able to inform this choice as subjects likely to be non-adherent may be excluded from such trials. On the other hand, observational studies often select PLWH already established on ART [5,6] where the observed effects of non-adherence on treatment outcome are likely to differ from those in individuals starting ART de novo. This selection bias may exclude those who have either experienced early virological failure, disease progression (or even death) or have defaulted from care. In addition, most studies either pre-date the use of boosted-PI regimens in first-line therapy [5,7] or include large numbers of people on unboosted PI regimens.

Three different outcomes may be considered: virological suppression, selection of drug resistance and effect of pattern of non-adherence.

\subsection{Effect of adherence on viral suppression}

There are no data from randomised controlled trials that directly address this question. Among subjects reporting $<95 \%$ adherence in a randomised controlled trial comparing lopinavir/r with once-daily darunavir/r, virological failure was more likely in the lopinavir/r than the darunavir/r arm; there were no differences between the two arms when analysing individuals reporting $>95 \%$ adherence [8].

Among individuals who were virologically suppressed initially, adherence $<95 \%$ was associated with an increased risk of failure [6], and very low adherence $(<50 \%)$ results in virological rebound irrespective of regimen $[6,9,10]$. However, virological suppression has been observed with only moderate adherence (50-75\%) among individuals on NNRTIs $[6,9,10]$ and virological failure has been reported to be significantly more likely on unboosted PI-based regimens where adherence was $<95 \%$ [6]. However, this finding may have been confounded by the once-daily dosing in the efavirenz group. A further study [11] examined only individuals with undetectable viraemia and found no difference in rates of virological rebound for those on PI/r vs. NNRTIs.

6.1.2.2.2 Effect of adherence on selection of drug resistance

The effect of level of non-adherence on selection of drug resistance varies by class. This was first described for unboosted PI regimens where moderate-to-high adherence was associated with increased risk of resistance [12]. The incidence of resistance in studies of boosted-PI regimens is low [8,13-17] but is observed with adherence just below $80-95 \%[5,18]$. In contrast, for first-generation NNRTIs the selection for resistance has been associated with very low average adherence $(<50 \%)[19,20]$.

\subsection{Effect of pattern of non-adherence}

The pattern of non-adherence may also be important. A number of small observational studies have examined short intermittent treatment interruptions (2-7 days) in individuals with prolonged virological suppression. For efavirenz, cycles of 2 days off per week appeared no more likely to result in treatment failure than continuous therapy, as long as the treatment interruption was not prolonged [21,22]. However, cycles of 7- or 28-day treatment interruption resulted in failure of efavirenz and selection of resistance [23,24]. For PI/r, one study found that average adherence, rather than duration of treatment interruption, was associated with virological response [25].

\subsubsection{References}

1 Gross R, Bilker WB, Friedman HM et al. Provider inaccuracy in assessing adherence and outcomes with newly initiated antiretroviral therapy. AIDS 2002; 16: 1835-1837.

2 Bangsberg DR, Hecht FM, Clague H et al. Provider assessment of adherence to HIV antiretroviral therapy. $J$ Acquir Immune Defic Syndr 2001; 26: 435-442.

3 Miller LG, Liu H, Hays RD et al. How well do clinicians estimate patients' adherence to combination antiretroviral therapy? J Gen Intern Med 2002; 17: 1-11.

4 Gardner EM, Burman WJ, Steiner JF et al. Antiretroviral medication adherence and the development of class-specific antiretroviral resistance. AIDS 2009; 23: 1035-1046.

5 Bangsberg DR, Acosta EP, Gupta R et al. Adherenceresistance relationships for protease and non-nucleoside reverse transcriptase inhibitors explained by virological fitness. AIDS 2006; 20: 223-231.

6 Maggiolo F, Airoldi M, Kleinloog HD et al. Effect of adherence to HAART on virologic outcome and on the selection of resistance-conferring mutations in NNRTI- or PI-treated patients. HIV Clin Trials 2007; 8: 282-292.

7 Trotta MP, Ammassari A, Cozzi-Lepri A et al. Adherence to highly active antiretroviral therapy is better in patients receiving non-nucleoside reverse transcriptase inhibitorcontaining regimens than in those receiving protease inhibitor-containing regimens. AIDS 2003; 17: 1099-1102.

8 Nelson M, Girard PM, Demasi R et al. Suboptimal adherence to darunavir/ritonavir has minimal effect on efficacy compared with lopinavir/ritonavir in treatment-naive, HIVinfected patients: 96 week ARTEMIS data. J Antimicrob Chemother 2010; 65: 1505-1509. 
9 Bangsberg DR. A paradigm shift to prevent HIV drug resistance. PLoS Med 2008; 5: e111.

10 Cambiano V, Lampe FC, Rodger AJ et al. Use of a prescription-based measure of antiretroviral therapy adherence to predict viral rebound in HIV-infected individuals with viral suppression. HIV Med 2010; 11: 216224.

11 Bangsberg DR. Less than 95\% adherence to nonnucleoside reverse-transcriptase inhibitor therapy can lead to viral suppression. Clin Infect Dis 2006; 43: 939-941.

12 Martin M, Del Cacho E, Codina C et al. Relationship between adherence level, type of the antiretroviral regimen, and plasma HIV type 1 RNA viral load: a prospective cohort study. AIDS Res Hum Retroviruses 2008; 24: 1263-1268.

13 Bangsberg DR, Hecht FM, Charlebois ED et al. Adherence to protease inhibitors, HIV-1 viral load, and development of drug resistance in an indigent population. AIDS 2000; 14: 357-366.

14 Kempf DJ, King MS, Bernstein B et al. Incidence of resistance in a double-blind study comparing lopinavir/ ritonavir plus stavudine and lamivudine to nelfınavir plus stavudine and lamivudine. J Infect Dis 2004; 189: 51-60.

15 Molina JM, Andrade-Villanueva J, Echevarria J et al. Oncedaily atazanavir/ritonavir versus twice-daily lopinavir/ ritonavir, each in combination with tenofovir and emtricitabine, for management of antiretroviral-naive HIV1-infected patients: 48 week efficacy and safety results of the CASTLE study. Lancet 2008; 372: 646-655.

16 Riddler SA, Haubrich R, DiRienzo AG et al. Class-sparing regimens for initial treatment of HIV-1 infection. $N$ Engl J Med 2008; 358: 2095-2106.

17 Walmsley S, Avihingsanon A, Slim J et al. Gemini: a noninferiority study of saquinavir/ritonavir versus lopinavir/ritonavir as initial HIV-1 therapy in adults. $J$ Acquir Immune Defic Syndr 2009; 50: 367-374.

18 Daar ES, Tierney C, Fischl MA et al. Atazanavir plus ritonavir or efavirenz as part of a 3-drug regimen for initial treatment of HIV-1. Ann Intern Med 2011; 154: 445-456.

19 Gardner EM, Sharma S, Peng G et al. Differential adherence to combination antiretroviral therapy is associated with virological failure with resistance. AIDS 2008; 22: 75-82.

20 King MS, Brun SC, Kempf DJ. Relationship between adherence and the development of resistance in antiretroviral-naive, HIV-1-infected patients receiving lopinavir/ritonavir or nelfinavir. J Infect Dis 2005; 191: 2046-2052.

21 Cohen CJ, Colson AE, Sheble-Hall AG et al. Pilot study of a novel short-cycle antiretroviral treatment interruption strategy: 48-week results of the five-days-on, two-days-off (FOTO) study. HIV Clin Trials 2007; 8: 19-23.

22 Reynolds SJ, Kityo C, Hallahan CW et al. A randomized, controlled, trial of short cycle intermittent compared to continuous antiretroviral therapy for the treatment of HIV infection in Uganda. PLoS One 2010; 5: e10307.

23 Dybul M, Nies-Kraske E, Daucher M et al. Long-cycle structured intermittent versus continuous highly active antiretroviral therapy for the treatment of chronic infection with human immunodeficiency virus: effects on drug toxicity and on immunologic and virologic parameters. $J$ Infect Dis 2003; 188: 388-396.

24 Parienti JJ, Ragland K, Lucht F et al. Average adherence to boosted protease inhibitor therapy, rather than the pattern of missed doses, as a predictor of HIV RNA replication. Clin Infect Dis 2010; 50: 1192-1197.

25 Ryan R, Santesso N, Hill S et al. Consumer-oriented interventions for evidence-based prescribing and medicines use: an overview of systematic reviews. Cochrane Database Syst Rev 2011; Cd007768.

\subsubsection{Dosing frequency}

An overview of systematic reviews of consumer-oriented medication interventions found that simplified dosing regimens improved adherence in the majority of studies in several reviews [1]. A review of adherence interventions for antiretroviral therapy included 19 studies (6312 adult individuals). Average adherence was modestly higher with once-daily regimens than twice-daily regimens (weighted mean difference $=2.55 \%$, 95\% CI 1.23 3.87, $P=0.0002$ ) but virological suppression was similar. Both adherence and viral suppression decreased over time, but adherence decreased less with once-daily than twice-daily dosing. Lower pill burden was associated with both better adherence and virological suppression [2]. NICE [3] reviewed several randomised controlled trials of interventions to reduce dose frequency and found that adherence may increase with once-daily dosing but not in all studies. Once-daily dosing is a reasonable intervention to reduce unintentional non-adherence to ART but no correpsonding impact on viral suppression has been observed.

\subsubsection{References}

1 Nieuwlaat R, Wilczynski N, Navarro T et al. Interventions for enhancing medication adherence. Cochrane Database Syst Rev 2014; 11: Cd000011.

2 Nachega JB, Parienti JJ, Uthman OA et al. Lower pill burden and once-daily antiretroviral treatment regimens for HIV infection: A meta-analysis of randomized controlled trials. Clin Infect Dis 2014; 58: 1297-1307.

3 National Collaborating Centre for Primary Care. Medicines adherence: Involving patients in decisions about prescribed medicines and supporting adherence. NICE Clinical Guideline 76 (CG76). 2009. Available at: http:// guidance.nice.org.uk/CG76 (accessed August 2015). 


\subsubsection{Fixed-dose combinations}

In examining whether fixed-dose combination formulations (FDCs) of drugs improve adherence or treatment outcome, only studies comparing the same drugs with the same dose frequency given as combination or separate pills were considered. No meta-analyses have been published on this subject for ART. A meta-analysis of nine randomised controlled trials and cohort studies in a range of diseases found the use of FDCs was associated with a significant reduction in the risk of non-adherence but in the single HIV randomised controlled trial included no significant difference in treatment failure between groups receiving FDC vs. non-FDC was observed [1]. Gupta et al. [2] reported a meta-analysis of cohort studies and found that use of FDCs for anti-hypertensives was associated with increased adherence but with no improvement on the control of blood pressure.

A retrospective study of a pharmacy database found no benefit in persistence on first-line ART for any FDC over separate agents [3]. In the ECHO/THRIVE studies, a lower virological response rate in individuals with baseline viral load 100,000-500,000 copies/mL was observed for rilpivirine- versus efavirenz-based regimens when dosed as separate agents [4]; this was not repeated when formulated as FDCs in the preliminary 48-week results from the STaR study [5]. Although the use of FDCs may have driven this apparent improvement in performance of rilpivirine, it may also have arisen due to the simpler oncedaily regimens in STaR, other methodological differences or by chance.

A further advantage of FDCs is that they prevent individuals from preferentially adhering less closely to one component of a regimen than others. A minority of participants in one study did report such 'differential' adherence, but this was not associated with outcome for currently used first-line strategies [6]. This was also reported in an Italian observational study; however, the difference was small and may have been confounded by other factors [7].

An observational study of outcomes following a switch from Atripla to multi-tablet regimens including swapping emtricitabine for lamivudine provides some evidence that this may not result in an increase in virological failures [8]. In view of the higher-quality evidence in support of FDCs and the implications and costs of treatment failure, there is insufficient evidence to support this strategy at present.

A recent meta-analysis of nine randomised-controlled antiretroviral trials analysed the impact of FDCs on adherence and treatment outcomes [9]. All nine trials were switch studies, regimen components differed between the FDC and non-FDC arms and five of the trials, investigating the impact of FDC backbone, were from several years ago. Four of the trials compared switching to a single-tablet regimen with continuing the existing multi-tablet regimen and there was no difference in virological failure rate; by switch = failure analysis there was a significant difference favouring single tablets.

In summary, FDCs support adherence to treatment, and this may well reduce the risk of virological failure. However, the size of this effect is yet to be defined. This needs to be balanced against the potentially far lower cost of generically available antiretroviral agents as separate or combined preparations of the same drugs.

\subsubsection{References}

1 Bangalore S, Kamalakkannan G, Parkar S, Messerli FH. Fixed-dose combinations improve medication compliance: a meta-analysis. Am J Med 2007; 120: 713-719.

2 Gupta AK, Arshad S, Poulter NR. Compliance, safety, and effectiveness of fixed-dose combinations of antihypertensive agents: a meta-analysis. Hypertension 2010; 55: 399-407.

3 Juday T, Grimm K, Zoe-Powers A et al. A retrospective study of HIV antiretroviral treatment persistence in a commercially insured population in the United States. AIDS Care 2011; 23: 1154-1162.

4 Cohen CJ, Molina JM, Cassetti I et al. Week 96 efficacy and safety of rilpivirine in treatment-naive, HIV-1 patients in two Phase III randomized trials. AIDS 2013; 27: 939-950.

5 Cohen C, Wohl D, Arribas J et al. STaR Study: single-tablet regimen emtricitabine/rilpivirine/tenofovir DF is noninferior to efavirenz/emtricitabine/tenofovir DF in ARTnai"ve adults week 48 results. 11th International Congress on Drug Therapy in HIV Infection. November 2012. Glasgow, Scotland [Abstract 425].

6 Gardner EM, Sharma S, Peng G et al. Differential adherence to combination antiretroviral therapy is associated with virological failure with resistance. AIDS 2008; 22: 75-82.

7 Fabbiani M, Grima P, Prosperi M et al. Single-tablet regimen is associated with reduced efavirenz withdrawal in antiretroviral naive or switching for simplification HIVinfected patients. Eleventh International Congress on Drug Therapy in HIV Infection. November 2012. Glasgow, Scotland [Abstract P11].

8 Engsig FN, Gerstoft J, Helleberg M et al. Effectiveness of antiretroviral therapy in individuals who for economic reasons were switched from a once-daily single-tablet regimen to a triple-tablet regimen. $J$ Acquir Immune Defic Syndr 2014; 66: 407-413.

9 Hill A, Pozniak A, Simmons B. No difference in risk of virological failure between antiretroviral treatments using co-formulated versus individual drugs: meta-analysis of 9 randomised trials in 2,568 patients. HIV Med 2015; 16 (Suppl 2): 1-11. 


\subsubsection{Single-tablet regimens}

There are currently four single-tablet regimens (STRs) available. Atripla (tenofovir-DF/emtricitabine/efavirenz) was the first STR approved for use in adults and in children at least 12 years old who weighed at least $40 \mathrm{~kg}$ [1]. The second available STR, which also contained an NNRTI, was Eviplera (tenofovir/emtricitabine/rilpivirine). Tenofovir-DF/emtricitabine/rilpivirine is licensed for the treatment of adults, who have no known mutations associated with resistance to NNRTIs, tenofovir-DF or emtricitabine, and have a viral load $\leq 100,000$ HIV-1 RNA copies/mL [2]. More recently, STRs containing an INSTI have become available. These are Stribild (tenofovir-DF/ emtricitabine/elvitegravir/c) [3] and Triumeq (abacavir/ lamivudine/dolutegravir) [4]. Triumeq is the first STR containing an abacavir/lamivudine backbone and testing for HLA-B*57:01 is recommended before use. If the individual is $\mathrm{HBV}$ co-infected, then additional drug treatment should be considered.

Potential advantages of using STRs are improved adherence, reduced selective adherence, patient preference and improvement in quality of life [5,6] although a recent analysis of individuals switching to STRs did not demonstrate significant changes in quality of life [7]. Randomised studies demonstrating these advantages are scarce, and among cohort and observation studies results are varied. One meta-analysis of four randomised switch STR trials has been presented but none of these trials compared the same drugs head to head [7]. In this metaanalysis there was no significant difference for virological failure, discontinuations due to adverse events or switch = failure endpoint. Only two of the trials assessed quality of life and found no difference between STR and control [7].

Disadvantages of STRs include cost, limited choice of regimens and the inability to dose-adjust for weight, renal impairment or drug-drug interactions. Also HLAB*57:01 testing and hepatitis B status must be considered for abacavir-containing STRs. Transmitted resistance will limit the possible use of STRs.

A cohort study from Denmark reported that switching from an STR to a generic triple-tablet regimen resulted in maintained efficacy, was safe and lower in cost [8]. A retrospective look at switching from FDCs to separate components in the Balearic Islands found lower pharmaceutical cost but higher overall healthcare cost in the first year following the change [9].

STRs have not been compared to the same drugs in multiple-pill regimens in randomised clinical trials. In the switch studies there was no proven benefit, despite the large cost difference. Although there are potential advantages of using STR there are currently no costeffectiveness data looking at STRs versus generic medications.

\subsubsection{References}

1 Electronic Medicines Compendium. SPC: Atripla $600 \mathrm{mg} /$ $200 \mathrm{mg} / 245 \mathrm{mg}$ film coated tablets. 2015. Available at: http://www.medicines.org.uk/EMC/medicine/20505/SPC/ Atripla+600+mg+200+mg+245+mg+film+coated+tablets/ (accessed August 2015).

2 Electronic Medicines Compendium. SPC: Eviplera $200 \mathrm{mg} /$ $25 \mathrm{mg} / 245 \mathrm{mg}$ film coated tablets. 2015. Available at: http://www.medicines.org.uk/EMC/medicine/25518/SPC/ Eviplera $+200+m g+25+m g+245+m g+$ film+coated+tablets/ (accessed August 2015).

3 Electronic Medicines Compendium. SPC: Stribild $150 \mathrm{mg} /$ $150 \mathrm{mg} / 200 \mathrm{mg} / 245 \mathrm{mg}$ film-coated tablets. 2015. Available at: https://www.medicines.org.uk/emc/medicine/27810/SPC/ Stribild $+150+m g+150+m g+200+m g+245+m g+f i l m-$ coated+tablets/

4 Electronic Medicines Compendium. SPC: Triumeq $50 \mathrm{mg}$, $300 \mathrm{mg}, 600 \mathrm{mg}$ Tablets. 2015. Available at: https:// www.medicines.org.uk/emc/medicine/29178 (accessed August 2015).

5 Astuti N, Maggiolo F. Single-tablet regimens in HIV therapy. Infect Dis Ther 2014; 3: 1-17.

6 Colombo GL, Di Matteo S, Antinori A et al. Economic evaluation of initial antiretroviral therapy for HIV-infected patients: an update of Italian guidelines. Clinicoecon Outcomes Res 2013; 5: 489-496.

7 Hill A, Pozniak A, Simmons B. No difference in risk of virological failure between antiretroviral treatments using co-formulated versus individual drugs: meta-analysis of 9 randomised trials in 2,568 patients. HIV Med 2015; 16 (Suppl 2): 1-11.

8 Engsig FN, Gerstoft J, Helleberg M et al. Effectiveness of antiretroviral therapy in individuals who for economic reasons were switched from a once-daily single-tablet regimen to a triple-tablet regimen. $J$ Acquir Immune Defic Syndr 2014; 66: 407-413.

9 Homar F, Lozano V, Martinez-Gomez J et al. Cost analysis of HIV treatment and drug-related adverse events when fixed-dose combinations of antiretrovirals (FDCs) were stopped, versus continuation with FDCs. Health Econ Rev 2012; 2: 16.

\subsection{Pharmacology}

More than for any other infection, individuals receiving ART require their doctor to have a clear understanding of the basic principles of pharmacology to ensure effective 
and appropriate prescribing. This is especially the case in four therapeutic areas.

\subsubsection{Drug interactions}

6.2.1.1 Recommendations

- Drug histories should be taken at each clinic visit, and a full medication history (including herbals, recreational drugs and other non-prescribed medications) should be undertaken at least annually (GPP).

- All potential adverse pharmacokinetic interactions between antiretroviral drugs and other concomitant medications should be checked before administration (with tools such as www.hiv-druginteractions.org) (GPP).

- Wherever feasible, PLWH should be counselled about the risks of drug interactions, and advised to use resources such as the Liverpool HIV Drug Interactions App (iOS or Android) (GPP).

6.2.1.2 Auditable measure

- Full medication history at least annually.

- Record in medical notes of potential adverse pharmacokinetic interactions between ARV drugs and other concomitant medications.

\subsubsection{Rationale}

The importance of eliciting a complete medication history in order to manage potential drug interactions in patients cannot be overemphasised. Drug-drug interactions may involve positive or negative interactions between ARV agents or between these and drugs used to treat other coexistent conditions. A detailed list is beyond the remit of these guidelines but clinically important interactions to consider when co-administering with ARV drugs include interactions with the following drugs: methadone, oral contraceptives, anti-epileptics, antidepressants, lipidlowering agents, acid-reducing agents, certain antimicrobials (e.g. clarithromycin, minocycline and fluconazole), some anti-arrhythmics, TB therapy, anti-cancer drugs, immunosuppressants, phosphodiesterase inhibitors and anti-hepatitis C virus (HCV) therapies. Most of these interactions can be managed safely (i.e. with/without dosage modification, together with enhanced clinical vigilance) but in some cases (e.g. rifampicin and PIs, proton pump inhibitors and atazanavir, inhaled fluticasone and ritonavir/cobicistat) the nature of the interaction is such that co-administration must be avoided and alternatives sought.

Importantly, education on the risks of drug interactions, including over-the-counter or recreational drugs, should be undertaken and PLWH should be encouraged to check with pharmacies or their healthcare professionals before commencing any new drugs, including those prescribed in primary care.
Large surveys report that about one-in-three-to-four PLWH receiving ART is at risk of a clinically significant drug interaction [1-7]. This suggests that safe management of HIV drug interactions is only possible if medication recording is complete, and if physicians are aware of the possibility that an interaction might exist. Incomplete or inaccurate medication recording has resulted from self-medication, between hospital and community health services [8] and within hospital settings particularly when multiple teams are involved, or when medical records are fragmented (e.g. with separate HIV case notes) [9].

More worryingly, one survey in the UK reported that even when medication recording is complete, physicians were only able to identify correctly one-third of clinically significant interactions involving HIV drugs [2]. In addition to HIV specialist and local drug information pharmacists, the University of Liverpool's comprehensive drug interaction website (www.hiv-druginteractions.org) is an excellent and highly recommended resource for information relating to potential drug interactions. Additional information resources also include the electronic medicines compendium (www.medicines.org.uk/emc) and medical information departments of pharmaceutical companies.

Communication with GPs and other medical specialties involved in care is fundamental in minimising the risk of adverse drug-drug interactions. All clinic letters should carry as a standard header or footer advice to check for interactions, and links to resources, such as www.hivdruginteractions.org, to address the potential for drug interactions.

\subsubsection{References}

1 Bias TE, Venugopalan V, Berkowitz LB, Cha A. Incidence of antiretroviral drug interactions during hospital course: the role of a pharmacist-led antiretroviral stewardship program. J Pharmacy Technol 2014; 30: 48-53.

2 Evans-Jones JG, Cottle LE, Back DJ et al. Recognition of risk for clinically significant drug interactions among HIVinfected patients receiving antiretroviral therapy. Clin Infect Dis 2010; 50: 1419-1421.

3 Kigen G, Kimaiyo S, Nyandiko W et al. Prevalence of potential drug-drug interactions involving antiretroviral drugs in a large Kenyan cohort. PLoS One 2011; 6: e16800.

4 Marzolini C, Elzi L, Gibbons S et al. Prevalence of comedications and effect of potential drug-drug interactions in the Swiss HIV Cohort Study. Antivir Ther 2010; 15: 413423.

5 Miller CD, El-Kholi R, Faragon JJ, Lodise TP. Prevalence and risk factors for clinically significant drug interactions with antiretroviral therapy. Pharmacotherapy 2007; 27: 13791386. 
6 Patel N, Abdelsayed S, Veve M, Miller CD. Predictors of clinically significant drug-drug interactions among patients treated with nonnucleoside reverse transcriptase inhibitor-, protease inhibitor-, and raltegravir-based antiretroviral regimens. Ann Pharmacother 2011; 45: 317-324.

7 Shah S, Shah S, McGowan J et al. Identification of drug interactions involving ART in New York City HIV specialty clinics. Conference on Retroviruses and Opportunistic Infections. February 2007. Los Angeles, CA, USA [Abstract 573].

8 de Maat MM, Frankfort SV, Mathot RA et al. Discrepancies between medical and pharmacy records for patients on antiHIV drugs. Ann Pharmacother 2002; 36: 410-415.

9 Seden K, Mathew T, Bradley M et al. Patients accessing HIV treatment via sexual health services: what are the risks of the dual case-note system? Int J STD AIDS 2012; 23: 99104.

\subsubsection{Therapeutic drug monitoring}

\subsubsection{Recommendation}

- We recommend against the unselected use of therapeutic drug monitoring (GPP).

- Therapeutic drug monitoring may be of clinical value in specific populations (e.g. children, pregnant women) or selected clinical scenarios (e.g. malabsorption, drug interactions, suspected non-adherence to therapy) (2C).

\subsubsection{Rationale}

Therapeutic drug monitoring (TDM) has been shown to be valuable in optimising the management of certain individuals; however, the general utility of this test in those receiving ART has been poorly assessed. With the marked improvement in efficacy and tolerability of modern ARV regimens, the role of TDM in clinical management has also evolved. A Cochrane review of randomised controlled trials [1] suggested little value when used unselectively. However, TDM may aid the management of vulnerable populations or complex clinical situations.

- Monitoring adherence. While detection of drug at therapeutic or even high plasma concentrations does not exclude low adherence, absence of measurable drug, or else very low levels of drug, strongly suggest lack of medication intake, particularly in the absence of evidence of significant malabsorption. Here, TDM should rarely be interpreted in isolation, but rather integrated with virological rebound, particularly in the absence of any resistance mutations and other features in the history that suggest risk for low treatment adherence.

- Optimising treatment in vulnerable PLWH. In vulnerable PLWH (e.g. children, pregnant women [2] and individuals with extremes of body mass index) or in specific clinical situations (e.g. liver and renal impairment, treatment failure, drug interactions both foreseen and unanticipated, malabsorption, suspected nonadherence and unlicensed once-daily dosing regimens). In these scenarios, the aim is to optimise dosing based either on known efficacy or toxicity cut-offs, or else to achieve the range of plasma concentrations encountered in individuals without these factors, who have been recruited to pharmacokinetic studies at licensed treatment doses that are known to be both safe and efficacious.

- Managing drug interactions (see above). Where the HIV drug has the potential to be adversely affected by another drug, and the combination is unavoidable, TDM may be used either to manage that interaction, or else discount a significant interaction in a particular individual.

- Other situations. Knowledge of plasma drug concentrations may be clinically useful when evaluating whether there is scope for treatment simplification, or else confirming or refuting impaired drug absorption as a reason for virological failure.

More detailed recommendations for the use of TDM are available in the BHIVA guidelines for the routine investigation and monitoring of adult HIV-1-infected individuals (www.bhiva.org/monitoring-guidelines.aspx). As for all other investigations, it is essential that TDM is undertaken correctly, especially with regard to timing (undertaken when steady state has been achieved). A consensus has been achieved for defining targets [3] for many ARVs. With many newer agents, evidence for a defined minimum target for efficacy is either weak or lacking, and evidence for an upper toxicity cut-off for most ARVs is lacking.

\subsubsection{References}

1 Kredo T, Van der Walt JS, Siegfried N, Cohen K. Therapeutic drug monitoring of antiretrovirals for people with HIV. Cochrane Database Syst Rev 2009; Cd007268.

2 Andany N, Loutfy MR. HIV protease inhibitors in pregnancy: pharmacology and clinical use. Drugs 2013; 73: 229-247.

3 La Porte C, Back D, Blaschke T et al. Updated guideline to perform therapeutic drug monitoring for antiretroviral agents. Rev Antivir Ther 2006; 3: 4-14.

\subsubsection{Stopping therapy: pharmacological considerations} 6.2.3.1 Recommendations

- We recommend individuals stopping ART containing an NNRTI in combination with an NRTI backbone replace all drugs with a PI (darunavir/r once daily) for 4 weeks (1C). 
- We recommend individuals stopping a PI-containing regimen stop all drugs simultaneously and no replacement is required $(1 \mathrm{C})$.

6.2.3.2 Auditable outcome

- Proportion of individuals with an undetectable viral load on ART who, on stopping a regimen containing an NNRTI in combination with an NRTI backbone, are switched to $\mathrm{PI} / \mathrm{r}$ for 4 weeks.

6.2.3.3 Rationale

In general, treatment interruptions are not recommended for most individuals. Whatever the reason for stopping ART (e.g. drug toxicity, intercurrent illness, after pregnancy or individual choice), pharmacological issues must be considered for a clinician to give guidance. The halflife of each drug included in the regimen is critical. There is the potential for monotherapy or dual therapy if ARV drugs with different half-lives are stopped simultaneously.

NNRTI and NRTI resistance mutations have been detected following discontinuation of previously suppressive regimens [1,2] and may have the potential to affect the likelihood of viral resuppression on restarting an NNRTI- based ART regimen.

There are limited data on which to base recommendations for how to protect against development of resistance in the period immediately following treatment cessation. Several discontinuation strategies have been proposed [3], and choice is influenced by clinical considerations, individual wishes and pharmacological principles. Options include: (i) simultaneously stopping all drugs in a regimen containing drugs with similar halflives; (ii) a staggered stop, discontinuing the drug with the longest half-life furst in a regimen containing drugs with short and long half-lives; or (iii) replacing all drugs with a drug with a short half-life and high genetic barrier to resistance (i.e. a PI). There is no randomised comparison of these three strategies. However, in one study a lower number of emergent resistance mutations were seen in those switching to a PI compared with those undertaking a simultaneous or staggered stop [2]. Therapeutic plasma concentrations of efavirenz can also be detected up to 3 weeks after stopping the drug in some people and thus a staggered stop of 1 week may potentially be inadequate to prevent emergence of NNRTI mutations [4]. The optimal duration of replacement with a PI is not known, but 4 weeks is probably advisable.

\subsubsection{References}

1 Cambiano V, Castro H, Chadwick D et al. Detection of NNRTI resistance mutations after*interrupting NNRTI-based regimens. Conference on Retroviruses and Opportunistic Infections. March 2014. Boston, MA, USA [Abstract 593].

2 Fox Z, Phillips A, Cohen C et al. Viral resuppression and detection of drug resistance following interruption of a suppressive non-nucleoside reverse transcriptase inhibitorbased regimen. AIDS 2008; 22: 2279-2289.

3 Taylor S, Boffito M, Khoo S et al. Stopping antiretroviral therapy. AIDS 2007; 21: 1673-1682.

4 Taylor S, Jayasuriya A, Fisher M et al. Lopinavir/ritonavir single agent therapy as a universal combination antiretroviral therapy stopping strategy: results from the STOP 1 and STOP 2 studies. J Antimicrob Chemother 2012; 67: 675-680.

\subsubsection{Switching therapy: pharmacological considerations}

\subsubsection{Recommendations}

There are no high-quality data on how to switch away from efavirenz to an alternative 'third' agent. Based on pharmacological principles, there is little rationale for any strategy other than straightforward substitution when switching to a PI/r or raltegravir. Pharmacokinetic studies show that straightforward substitution with etravirine, rilpivirine, dolutegravir and elvitegravir/c may result in slightly lower concentrations of either drug for a short period following switching, but limited virological data suggest that risk of virological failure with this strategy is low. Different strategies for switching to nevirapine have been proposed, but no comparative data are available to guide the choice of strategy. Limited data suggest that the dose of maraviroc should be doubled in the week following switching (unless given together with a PI/r).

If switching away from efavirenz is undertaken when viral load is likely to still be detectable (e.g. because of CNS intolerance within the first few weeks of starting efavirenz), substitution with a $\mathrm{PI} / \mathrm{r}$ in preference to a within-class switch is advised.

6.2.4.2 Rationale

Switching a component of an ART regimen is frequently considered in PLWH to manage drug side effects or address adherence issues. ARVs that either induce or inhibit drug-metabolising enzymes have the potential to affect the plasma concentrations of the new agent. This applies in particular to switching away from NNRTIs. Induction of drug metabolising enzymes by efavirenz is likely to persist for a period beyond drug cessation. Consideration should also be given of whether or not viral load is maximally suppressed when planning how to switch away from efavirenz to an alternative agent. Broadly, strategies for switching from efavirenz to an alternative 'third' agent may be summarised as follows. 


\subsection{Efavirenz to nevirapine}

A pharmacokinetic study performed in HIV-positive individuals suggested that individuals changing from efavirenz to nevirapine should commence on $200 \mathrm{mg}$ twice a day to ensure therapeutic plasma concentrations and potentially avoid selection of resistance to nevirapine [1]. However, no one in the nevirapine lead-in group experienced virological failure in the 3-month follow-up period. Switching without dose escalation is in direct contrast to the information in the Viramune summary of product characteristics, which advises administration of a nevirapine lead-in dose (200 $\mathrm{mg}$ once daily for 2 weeks) when starting nevirapine [2], as this has been shown to decrease the frequency of rash.

In ART-experienced individuals who are virologically suppressed with an undetectable plasma HIV RNA level $(<50$ copies/mL), the risk of hypersensitivity and/or hepatotoxicity on switching to nevirapine is not increased in those with higher CD4 cell counts (above the gender-specific CD4 cell count thresholds) [3]. In ART-experienced individuals with detectable plasma HIV RNA levels, a switch to nevirapine is not advised.

Furthermore, the need to minimise any window for developing resistance is greatest in those who discontinue efavirenz early on when virological suppression has not yet been achieved. The latter scenario is made more complex when enzyme induction has not yet been fully achieved, and if doubt exists, alternatives to switch to should be considered.

6.2.4.2.2 Efavirenz to etravirine

In individuals with undetectable viral loads, switching from efavirenz to etravirine, standard doses of etravirine can be commenced [4]. To date, no data are available on what strategy to adopt in those with active viral replication.

6.2.4.2.3 Efavirenz to rilpivirine

Concentrations of rilpivirine are lowered by previous efavirenz administration. However, 28 days after the switch, they returned to levels comparable with those when rilpivirine was administered without previous efavirenz treatment, except for a 25\% lower $\mathrm{C}_{\min }$. Therefore, for individuals with undetectable viral loads switching from efavirenz to rilpivirine, standard doses of rilpivirine can be commenced [5]. To date, no data are available on what strategy to adopt in individuals with active viral replication.

6.2.4.2.4 Efavirenz to a ritonavir-boosted protease inhibitor

Because of the strong inhibitory effect of ritonavir on CYP450 3A4, a modification of the PI/r dose is unlikely to be needed when switching from efavirenz to PI/r. Formal pharmacokinetic data are unavailable. TDM data on atazanavir/r showed that after stopping efavirenz, atazanavir concentrations were above the suggested minimum effective concentration in all studied subjects [6].

\subsection{Efavirenz to raltegravir}

Although formal pharmacokinetic data are not available, switching efavirenz to raltegravir should not lead to clinically significant consequences, as co-administration of efavirenz with raltegravir led to a $21 \%$ decrease in raltegravir $\mathrm{C}_{\min }$ and the degree of this reduction is unlikely to be clinically meaningful [7].

6.2.4.2.6 Efavirenz to maraviroc

A formal pharmacokinetic study in HIV-positive individuals showed that the induction effect of efavirenz necessitated an increase in maraviroc dose to $600 \mathrm{mg}$ twice daily for 1 week following the switch [8]. Maraviroc $300 \mathrm{mg}$ twice daily (standard dose) seems to be safe after this period. Although there is an absence of data, when switching from efavirenz to maraviroc plus a $\mathrm{PI} / \mathrm{r}$, it is likely that a dose of $150 \mathrm{mg}$ twice daily is safe from the first day after the switch. Whether it is advisable to use maraviroc $150 \mathrm{mg}$ once daily in this context or for how long a twice-daily dose should be used after the switch remains unknown.

\subsection{Efavirenz to elvitegravir/cobicistat}

Concentrations of elvitegravir are lowered by previous efavirenz administration: elvitegravir $\mathrm{AUC} / \mathrm{C}_{\text {trough }}$ were 37\%/67\% and 29\%/55\% lower 1 and 2 weeks postswitch, respectively, but still above the protein bindingadjusted 95\% inhibitory concentration (45 ng/mL). Therefore, for individuals with undetectable viral loads switching from efavirenz to elvitegravir/c, standard doses of elvitegravir/c can be commenced [9]. Pharmacodynamic data from a Phase $3 \mathrm{~b}$ switch study in HIV-positive subjects are also available [10]. To date, no data are available on what strategy to adopt in individuals with active viral replication.

\subsection{Efavirenz to dolutegravir}

Mathematical modelling suggests that dolutegravir concentrations achieved target minimum effective concentrations by 3 days following efavirenz discontinuation (6 days in efavirenz poor metabolisers). Importantly, there was no time point where both the efavirenz and dolutegravir concentrations were predicted to be sub-therapeutic [11]. On that basis, straightforward (with no dose adjustment) substitution of efavirenz with dolutegravir is recommended.

\subsection{Nevirapine to rilpivirine}

A study switching 32 individuals with an undetetcbale viral load from tenofovir-DF/emtricitabine + nevirapine to tenofovir-DF/emtricitabine/rilpivirine fixed-dose combination showed that all individuals maintained viral suppression. Mean rilpivirine $\mathrm{C}_{\text {trough }}$ was above $\mathrm{C}_{\text {trough }}$ 
measured in Phase 3 studies by week 1 and the majority of individuals (27/32) had undetectable plasma nevirapine concentrations by week 2 [12]. Therefore, for individuals with undetectable viral loads switching from nevirapine to rilpivirine, standard doses of rilpivirine can be commenced. To date, no data are available on what strategy to adopt in individuals with active viral replication.

\subsection{Nevirapine to dolutegravir}

Concentrations of dolutegravir have been shown to be moderately decreased $(n=10)$ by previous nevirapine administration in PLWH with an undetectable viral load: dolutegravir AUC was lowered by $19 \%$ and $\mathrm{C}_{\text {trough }}$ by 34\% [13] These changes are unlikely to be clinically significant as dolutegravir concentrations are maintained well above the protein binding-adjusted 90\% inhibitory concentration (64 $\mathrm{ng} / \mathrm{mL}$ ) during and right after stopping nevirapine administration. Therefore, for individuals with undetectable viral loads switching from nevirapine to rilpivirine, standard doses of rilpivirine can be commenced.

\subsubsection{References}

1 Winston A, Pozniak A, Smith N et al. Dose escalation or immediate full dose when switching from efavirenz to nevirapine-based highly active antiretroviral therapy in HIV-1-infected individuals? AIDS 2004; 18: 572-574.

2 Boehringer Ingelheim. Viramune Summary of Product Characteristics. 2011.

3 Kesselring AM, Wit FW, Sabin CA et al. Risk factors for treatment-limiting toxicities in patients starting nevirapinecontaining antiretroviral therapy. AIDS 2009; 23: 16891699.

4 Waters L, Fisher M, Winston A et al. A phase IV, double-blind, multicentre, randomized, placebo-controlled, pilot study to assess the feasibility of switching individuals receiving efavirenz with continuing central nervous system adverse events to etravirine. AIDS 2011; 25: 65-71.

5 Crauwels H, Vingerhoets J, Ryan R et al. Pharmacokinetic parameters of once-daily TMC278 following administration of EFV in healthy volunteers. Conference on Retroviruses and Opportunistic Infections. February 2011. Boston, MA, USA. [Abstract 630].

6 Maitland D, Boffito M, Back D et al. Therapeutic drug monitoring (TDM) of atazanavir (ATV) during the first 4 weeks of therapy after switching from efavirenz (EFV) containing regimen. 7th International Congress on Drug Therapy in HIV Infection. November 2004. Glasgow, Scotland [Abstract 293].

7 Iwamoto M, Wenning LA, Petry AS et al. Minimal effects of ritonavir and efavirenz on the pharmacokinetics of raltegravir. Antimicrob Agents Chemother 2008; 52: 43384343.
8 Waters L, Jackson A, Else L et al. Switching safely: pharmacokinetics, efficacy and safety of switching efavirenz to maraviroc twice daily in patients on suppressive antiretroviral therapy. Antivir Ther 2015; 20: 157-163.

9 Cohen C, Elion R, Ruane P et al. Switch from efavirenz/ emtricitabine/tenofovir DF to elvitegravir/cobicistat/ emtricitabine/tenofovir DF: efficacy and pharmacokinetics. 53rd ICAAC. September 2013. Denver, CO, USA [Abstract H658].

10 Pozniak A, Markowitz M, Mills A et al. Switching to coformulated elvitegravir, cobicistat, emtricitabine, and tenofovir versus continuation of non-nucleoside reverse transcriptase inhibitor with emtricitabine and tenofovir in virologically suppressed adults with HIV (STRATEGYNNRTI): 48 week results of a randomised, open-label, phase 3b non-inferiority trial. Lancet Infect Dis 2014; 14: 590599.

11 Generaux G, Song I, Bowers G, Piscitelli S. A mechanistic SimCYP simulation evaluating dolutegravir and efavirenz pharmacokinetics following a switch from once-daily efavirenz to once-daily dolutegravir. 15th International Workshop on Clinical Pharmacology of HIV and Hepatitis Therapy. May 2014. Washington DC, USA [Abstract P_36].

12 Allavena C, Dailly E, Reliquet V et al. Switching from tenofovir/emtricitabine and nevirapine to a tenofovir/ emtricitabine/rilpivirine single-tablet regimen in virologically suppressed, HIV-1-infected subjects. $J$ Antimicrob Chemother 2014; 69: 2804-2808.

13 Dailly E, Allavena C, Gregoire M et al. Influence of nevirapine administration on the pharmacokinetics of dolutegravir in patients infected with HIV-1. J Antimicrob Chemother 2015.

\subsection{Switching antiretroviral therapy in virological suppression}

\subsubsection{Introduction}

In individuals on fully virally suppressive regimens, switching individual components of the ART combination regimen is frequently considered for several reasons, including: management of ARV drug toxicity or intolerance, desire for once-daily dosing and reduced pill burden, management of potential drug-drug interactions, individual preference and cost [1]. Guidance on the management of drug toxicity of individual ARVs is not within the scope of these guidelines. Guidance on interventions to support adherence, including once-daily dosing and fixed-dose combinations is addressed in Section 6.1 (Adherence) and pharmacological considerations on switching ARVs is discussed in Section 6.2.4.

Switching individual components of an ART regimen may well improve adherence and tolerability, but should 
not be at the cost of virological efficacy. The following guidance concerns the impact on virological efficacy of either switching the third agent or the NRTI backbone in a combination ART regimen or simplifying to boosted PI monotherapy. Evidence from a systematic literature review (Appendix 2) was evaluated as well as the impact on critical treatment outcomes of the different switching strategies assessed. Critical outcomes included virological suppression at 48 weeks, virological failure and discontinuation from grade $3 / 4$ events. Of note, when switching in the context of viral suppression, rilpivirine and abacavir/lamivudine can be used regardless of pre-treatment viral load.

\subsubsection{Switching antiretrovirals in combination antiretroviral therapy \\ 6.3.2.1 Recommendations}

- We recommend, in individuals on suppressive ART regimens, consideration is given to differences in sideeffect profile, drug-drug interactions and drug-resistance patterns before switching any ARV component (GPP).

- In individuals with previous NRTI resistance mutations, we recommend against switching a PI/r to either an NNRTI or an INI as the third agent (1B).

6.3.2.2 Auditable outcome

- Number of individuals with an undetectable viral load on current regimen and documented previous NRTI resistance who have switched a PI/r to either an NNRTI or INI as the third agent.

6.3.2.3 Rationale

Within-class switches are usually undertaken to improve ARV tolerability or to address potential adverse effects. The available evidence for current recommended third agents is limited but switching PI/r or NNRTIs in virologically suppressed individuals has, in a small number of studies, not been associated with loss of virological efficacy [2-4]. Consideration should, however, be given to differences in side-effect profiles, drug-drug interactions, pill burden and food effect (see Appendix 4). When switching between different PIs, a previous history of major PI mutations may potentially have an adverse effect on the virological efficacy of the new PI/r.

\subsection{Within class}

For NRTIs, recent studies have mainly evaluated switching from a thymidine analogue to either tenofovir-DF or abacavir to manage lipoatrophy, or have investigated switching to one of two available NRTI fixed-dose combinations (FDC; tenofovir-DF and emtricitabine or abacavir and lamivudine). If screening for HLA-B*57:01 positivity is undertaken before the switch to abacavir, then similar virological efficacy is seen in subjects switched to abacavir/lamivudine FDC compared with a switch to tenofovir-DF/emtricitabine FDC [5]. Switching from abacavir/emtricitabine FDC to tenofovir-DF/emtricitabine FDC was also found to maintain viral suppression and is safe [6]. However, switching to simplify to a triple nucleoside regimen is not recommended [7]. For PIs, recent studies have evaluated both within-class switching and simplification to an unboosted regimen [8-12]. These changes have been to reduce bilirubin, lipids or bone and renal biomarkers. For NNRTIs, etravirine and rilpivirine have been evaluated in an intraclass switch [4,13,14]. All study participants maintained virological control and there were no adverse events. With the exception of a small, single arm tenofovir-DF/emtricitabine plus raltegravir to Stribild switch study (50 subjects, all of whom maintained virological suppression with minimal impact on renal and lipid markers) [15], there are no integrase inhibitor within-class switch studies. In general, in the absence of previous resistance mutations, switching within class should result in maintaining virological suppression.

\subsection{Switch from PI}

Several randomised controlled trials have assessed switching between classes (PI to NNRTI or INI, NNRTI to INI) in individuals who are virologically suppressed. A meta-analysis of six trials showed non-inferiority in maintenance of virological suppression when switching from a PI (both ritonavir boosted and unboosted) to nevirapine compared with continuing the PI, but was associated with more discontinuations due to liver toxicity [16]. Previous treatment failure on an NRTI-containing regimen has been associated with an increased risk of virological failure when switching from a PI- to an NNRTIbased regimen [17]. A cohort analysis showed similar rates of virological failure at 12 months in PLWH switching from a first-line PI/r to either efavirenz or nevirapine compared with continuing on the PI/r [18]. If switching to nevirapine, consideration should be given to the risk of hypersensitivity reactions and hepatotoxicity. Similar rates have been reported in virologically suppressed compared with ART-naïve individuals stratified for CD4 cell count and gender [19,20]. Only one randomised controlled trial has assessed the switch from PI to once-daily etravirine in people with HIV RNA suppression [21] and no participants presented with virological failure through to 48 weeks. Switching in virological suppression to rilpivirine from PI-maintained suppression was safe and, with or without K103N, had a high response rate [22-24]. For individuals without previous NRTI or NNRTI resistance mutations switching from a PI/r to any of the current licensed NNRTIs is likely to maintain virological efficacy 
and choice of NNRTI will depend on side-effect profile, tolerability and individual preference.

Switching from a PI/r to the INI, raltegravir, in virologically suppressed individuals has been evaluated in three randomised controlled trials. Two studies have shown that previous history of NRTI resistance mutations increases the risk of subsequent virological failure on switching compared with continuing on a PI/r [25,26]. This association was not seen in a third trial [6]. However, it is not surprising that switching from an ARV with a high genetic barrier to one with a low genetic barrier to resistance may potentially increase the risk of virological failure if the activity of the NRTI backbone has been compromised by previous NRTI resistance. One randomised controlled trial assessed switching from PI to elvitegravir/c in people with viral suppression (excluding individuals with a history of virological failure or resistance to tenofovir-DF or emtricitabine), finding suppression is maintained and regimen is well tolerated [27].

6.3.2.3.3 Switch from NNRTI

Switching from an NNRTI to an alternative third agent (elvitegravir/c or raltegravir [28,29]) in virologically suppressed patients has been assessed. Raltegravir was assessed for patient preference and was found to be acceptable. NNRTI switch to elvitegravir/c maintained viral suppression and was well tolerated. If switching from an NNRTI, consideration must be given to previous treatment history and potential pharmacokinetic interactions. The latter is discussed in more detail in Section 6.2.4.

\subsubsection{References}

1 Vo TT, Ledergerber B, Keiser 0 et al. Durability and outcome of initial antiretroviral treatments received during 2000-2005 by patients in the Swiss HIV Cohort Study. $J$ Infect Dis 2008; 197: 1685-1694.

2 Mallolas J, Podzamczer D, Milinkovic A et al. Efficacy and safety of switching from boosted lopinavir to boosted atazanavir in patients with virological suppression receiving a LPV/r-containing HAART: the ATAZIP study. J Acquir Immune Defic Syndr 2009; 51: 29-36.

3 Soriano V, Garcia-Gasco P, Vispo E et al. Efficacy and safety of replacing lopinavir with atazanavir in HIV-infected patients with undetectable plasma viraemia: final results of the SLOAT trial. J Antimicrob Chemother 2008; 61: 200205.

4 Waters L, Fisher M, Winston A et al. A phase IV, doubleblind, multicentre, randomized, placebo-controlled, pilot study to assess the feasibility of switching individuals receiving efavirenz with continuing central nervous system adverse events to etravirine. AIDS 2011; 25: 65-71.
5 Martin A, Bloch M, Amin J et al. Simplification of antiretroviral therapy with tenofovir-emtricitabine or abacavir-Lamivudine: a randomized, 96-week trial. Clin Infect Dis 2009; 49: 1591-1601.

6 Martinez E, Larrousse M, Llibre JM et al. Substitution of raltegravir for ritonavir-boosted protease inhibitors in HIVinfected patients: the SPIRAL study. AIDS 2010; 24: 16971707.

7 Campo R, DeJesus E, Bredeek UF et al. SWIFT: prospective 48-week study to evaluate efficacy and safety of switching to emtricitabine/tenofovir from lamivudine/abacavir in virologically suppressed HIV-1 infected patients on a boosted protease inhibitor containing antiretroviral regimen. Clin Infect Dis 2013; 56: 1637-1645.

8 Baril J, Conway B, Giguere P et al. A meta-analysis of the efficacy and safety of unboosted atazanavir compared with ritonavir-boosted protease inhibitor maintenance therapy in HIV-infected adults with established virological suppression after induction. HIV Med 2014; 15: 301-310.

9 Cruciani M, Mengoli C, Serpelloni G et al. Abacavir-based triple nucleoside regimens for maintenance therapy in patients with HIV. Cochrane Database Syst Rev 2013; 6: Cd008270.

10 Ferraris L, Vigano 0, Peri A et al. Switching to unboosted atazanavir reduces bilirubin and triglycerides without compromising treatment efficacy in UGT1A $1 * 28$ polymorphism carriers. J Antimicrob Chemother 2012; 67 : 2236-2242.

11 Ferrer E, del Rio L, Martinez E et al. Impact of switching from lopinavir/ritonavir to atazanavir/ritonavir on body fat redistribution in virologically suppressed HIV-infected adults. AIDS Res Hum Retroviruses 2011; 27: 1061-1065.

12 Wohl DA, Bhatti L, Small CB et al. Simplification to abacavir/lamivudine + atazanavir maintains viral suppression and improves bone and renal biomarkers in ASSURE, a randomized, open label, non-inferiority trial. PLoS One 2014; 9: e96187.

13 Skiest DJ, Calvin Cohen C, Khanlou H et al. Efficacy and safety of switching suppressed patients with elevated triglycerides from lopinavir/ritonavir or fosamprenavir/ ritonavir to atazanavir/ritonavir or darunavir/ritonavir based therapy: The LARD study. HIV AIDS Rev 2012; 11: 77-83.

14 Nelson M, Winston A, Waters L et al. Multicentre openlabel study of switching from Atripla to Eviplera for possible efavirenz associated CNS toxicity. 53rd Interscience Conference on Antimicrobial Agents and Chemotherapy. September 2013. Denver, CO, USA [Abstract H-672b].

15 Mills A, Crofoot G, Ortiz R et al. Switching from twice-daily raltegravir plus tenofovir disoproxil fumarate/emtricitabine to once-daily elvitegravir/cobicistat/emtricitabine/tenofovir disoproxil fumarate in virologically suppressed, HIV-1- 
infected subjects: 48 weeks data. HIV Clin Trials 2014; 15: 51-56.

16 Ena J, Leach A, Nguyen P. Switching from suppressive protease inhibitor-based regimens to nevirapine-based regimens: a meta-analysis of randomized controlled trials. HIV Med 2008; 9: 747-756.

17 Martinez E, Arnaiz JA, Podzamczer D et al. Substitution of nevirapine, efavirenz, or abacavir for protease inhibitors in patients with human immunodeficiency virus infection. $N$ Engl J Med 2003; 349: 1036-1046.

18 Bommenel T, Launay 0, Meynard JL et al. Comparative effectiveness of continuing a virologically effective first-line boosted protease inhibitor combination or of switching to a three-drug regimen containing either efavirenz, nevirapine or abacavir. J Antimicrob Chemother 2011; 66: 1869-1877.

19 Kesselring AM, Wit FW, Sabin CA et al. Risk factors for treatment-limiting toxicities in patients starting nevirapinecontaining antiretroviral therapy. AIDS 2009; 23: 16891699.

20 Wit FW, Kesselring AM, Gras L et al. Discontinuation of nevirapine because of hypersensitivity reactions in patients with prior treatment experience, compared with treatmentnaive patients: the ATHENA cohort study. Clin Infect Dis 2008; 46: 933-940.

21 Echeverria P, Bonjoch A, Puig J et al. Randomised study to assess the efficacy and safety of once-daily etravirine-based regimen as a switching strategy in HIV-infected patients receiving a protease inhibitor-containing regimen. Etraswitch study. PLoS One 2014; 9: e84676.

22 Flamm J, Shamblaw D, Ruane P et al. Comparison of pharmacy costs after switching to emtricitabine/rilpivirine/ tenofovir DF single-tablet regimen from a ritonavir-boosted protease inhibitor and 2 nucleoside reverse transcriptase inhibitors. J Managed Care Pharmacy 2012; 18: 544.

23 Palella FJ Jr, Fisher M, Tebas P et al. Simplification to rilpivirine/emtricitabine/tenofovir disoproxil fumarate from ritonavir-boosted protease inhibitor antiretroviral therapy in a randomized trial of HIV-1 RNA-suppressed participants. AIDS 2014; 28: 335-344.

24 Pallela F, Tebas P, Fisher M et al. Efficacy of switching to rilpivirine/emtricitabine/tenofovir DF from boosted PI in HIV-1 virologically suppressed patients with or without the K103N. HIV Drug Therapy in the Americas Congress. June 2013. Sao Paulo, Brazil [Abstract P25].

25 Eron JJ, Young B, Cooper DA et al. Switch to a raltegravirbased regimen versus continuation of a lopinavir-ritonavirbased regimen in stable HIV-infected patients with suppressed viraemia (SWITCHMRK 1 and 2): two multicentre, double-blind, randomised controlled trials. Lancet 2010; 375: 396-407.
26 Vispo E, Barreiro P, Maida I et al. Simplification from protease inhibitors to once- or twice-daily raltegravir: the ODIS trial. HIV Clin Trials 2010; 11: 197-204.

27 Fisher M, Nelson M, Johnson M et al. Simplification of $\mathrm{PI}+\mathrm{RTV}+\mathrm{FTC} / \mathrm{TDF}$ to $\mathrm{E} / \mathrm{C} / \mathrm{F} / \mathrm{TDF}$ maintains HIV suppression and is well tolerated. HIV Med 2014; 15 (Suppl 3): 114.

28 Nguyen A, Calmy A, Delhumeau C et al. A randomized crossover study to compare efavirenz and etravirine treatment. AIDS 2011; 25: 57-63.

29 Pozniak A, Kegg S, Fisher M et al. Switch from NNRTI plus FTC/TDF to E/C/F/TDF maintains HIV suppression and is well tolerated. HIV Med 2014; 15 (Suppl 3): 114.

\subsubsection{Protease inhibitor monotherapy}

6.3.3.1 Recommendations

- We recommend against the use of PI monotherapy for routine ART (1A).

- We recommend against the use of PI monotherapy for individuals whose initial regimen has failed or who have established resistance to one more antiretroviral drugs (1A).

6.3.3.2 Auditable outcome

- Proportion of individuals on PI/r monotherapy as ART maintenance strategy and record of rationale.

6.3.3.3 Rationale

For the assessment and evaluation of evidence, GRADE tables were constructed (Appendix 3). Virological suppression, drug resistance and serious adverse events were defined as critical outcomes. From the systematic literature review (Appendix 2), twelve randomised trials and two meta-analyses were identified, investigating the use of either lopinavir/r or darunavir/r in stable, virologically suppressed PLWH without active hepatitis B co-infection [1-14]. Assessment of virological suppression showed significantly fewer on PI monotherapy maintained virological suppression compared with those continuing on standard combination ART (RR 0.95, 95\% CI 0.9-0.99), although the difference was small. Viral load rebound is usually at low level, and is easily reversed by reintroduction of NRTIs [10]. There were no differences in the frequency of emergence of viral resistance, or of serious adverse events. The long-term consequences of this viral rebound and resuppression were assessed in the PIVOT trial where primary endpoint was loss of future drug options and secondary endpoints included clinical events [15]. PI monotherapy was non-inferior (inferiority margin $10 \%$ ) on the primary end point of impact on future treatment options [15]. One potential concern is the development of CNS disease in people on PI monotherapy $[7,14,15]$; however, PIVOT did not identify any difference in neurocognitive scoring over 5 years [15]. Overall, there is no significant clinical benefit of PI monotherapy 
compared with standard combination ART and the added disadvantage of a lower rate of viral suppression with PI monotherapy. For this reason PI monotherapy should not be used in unselected populations for maintaining virological suppression where standard ART is an acceptable alternative. There may be potential benefits of PI monotherapy, in terms of drug resistance, long-term drug toxicity and cost $[16,17]$. We recognise that PI monotherapy may well be an acceptable option in some specific populations and has been found to be safe, well tolerated and cost-effective [15]. Clinicians might consider PI monotherapy in individuals who are unable to tolerate NRTIs due to toxicities or as a short-term measure to manage or bridge complex clinical scenarios (e.g. stopping certain NNRTI-containing regimens or managing toxicity overdose or acute illness). Where PI monotherapy is considered, darunavir/r (dosed once or twice daily) or lopinavir/r (dosed twice daily) should be used but with reintroduction of NRTIs if there is loss of virological control. Atazanavir/r monotherapy is not recommended because it has been associated with higher rates of virological failure $[18,19]$. PI monotherapy is not recommended in individuals with active hepatitis B coinfection.

6.3.3.3.1 PI monotherapy as second line after first-line treatment failure

Three randomised controlled trials looked at PI monotherapy as second-line therapy compared to triple-ART in treatment failure, all in an African setting. In all three trials the PI monotherapy arm had significantly more participants with virological failure than the triple ART or other arms in the trials [20-22].

This strategy therefore is not recommended.

\subsubsection{References}

1 Arribas JR, Delgado R, Arranz A et al. Lopinavir-ritonavir monotherapy versus lopinavir-ritonavir and 2 nucleosides for maintenance therapy of HIV: 96-week analysis. J Acquir Immune Defic Syndr 2009; 51: 147-152.

2 Arribas JR, Horban A, Gerstoft J et al. The MONET trial: darunavir/ritonavir with or without nucleoside analogues, for patients with HIV RNA below 50 copies/mL. AIDS 2010; 24: 223-230.

3 Arribas JR, Pulido F, Delgado R et al. Lopinavir/ritonavir as single-drug therapy for maintenance of HIV-1 viral suppression: 48-week results of a randomized, controlled, open-label, proof-of-concept pilot clinical trial (OK Study). $J$ Acquir Immune Defic Syndr 2005; 40: 280-287.

4 Pulido F, Arribas JR, Delgado R et al. Lopinavir-ritonavir monotherapy versus lopinavir-ritonavir and two nucleosides for maintenance therapy of HIV. AIDS 2008; 22: F1-9.
5 Arribas JR, Clumeck N, Nelson M et al. The MONET trial: week 144 analysis of the efficacy of darunavir/ritonavir (DRV/r) monotherapy versus DRV/r plus two nucleoside reverse transcriptase inhibitors, for patients with viral load < 50 HIV-1 RNA copies/mL at baseline. HIV Med 2012; 13: 398-405.

6 Cahn P, Montaner J, Junod P et al. Pilot, randomized study assessing safety, tolerability and efficacy of simplified LPV/r maintenance therapy in HIV patients on the 1 PI-based regimen. PLoS One 2011; 6: e23726.

7 Gutmann C, Cusini A, Gunthard HF et al. Randomized controlled study demonstrating failure of LPV/r monotherapy in HIV: the role of compartment and CD4nadir. AIDS 2010; 24: 2347-2354.

8 Hasson H, Galli L, Gallotta G et al. HAART simplification with lopinavir/ritonavir monotherapy in HIV/HCV coinfected patients starting anti-HCV treatment: a randomised pilot study (KaMon study). New Microbiol 2012; 35: 469474.

9 Katlama C, Valantin MA, Algarte-Genin M et al. Efficacy of darunavir/ritonavir maintenance monotherapy in patients with HIV-1 viral suppression: a randomized open-label, noninferiority trial, MONOI-ANRS 136. AIDS 2010; 24: 2365-2374.

10 Mathis S, Khanlari B, Pulido F et al. Effectiveness of protease inhibitor monotherapy versus combination antiretroviral maintenance therapy: a meta-analysis. PLoS One 2011; 6: e22003.

11 Meynard JL, Bouteloup V, Landman R et al. Lopinavir/ ritonavir monotherapy versus current treatment continuation for maintenance therapy of HIV-1 infection: the KALESOLO trial. J Antimicrob Chemother 2010; 65: 2436-2444.

12 Nunes EP, Santini de Oliveira M, Mercon M et al. Monotherapy with Lopinavir/Ritonavir as maintenance after HIV-1 viral suppression: results of a 96-week randomized, controlled, open-label, pilot trial (KalMo study). HIV Clin Trials 2009; 10: 368-374.

13 Waters L, Jackson A, Singh K et al. The impact of continued ART versus lopinavir/ritonavir monotherapy $(\mathrm{mLPV} / \mathrm{r})$ on body fat and bone mineral density (BMD) as measured by DEXA: 48 week results of a randomised study. XVII International AIDS Conference. August 2008. Mexico City, Mexico [Abstract CDB0193].

14 Winston A, Fatkenheuer G, Arribas J et al. Neuropsychiatric adverse events with ritonavir-boosted darunavir monotherapy in HIV-infected individuals: a randomised prospective study. HIV Clin Trials 2010; 11: 163-169.

15 Paton N, Stohr W, Arenas-Pinto A et al. The protease inhibitor versus ongoing triple-therapy (PIVOT) trial. HIV Med 2014; 15 (Suppl 3): 1. 
16 Gazzard B, Hill A, Anceau A. Cost-efficacy analysis of the MONET trial using UK antiretroviral drug prices. Appl Health Econ Health Policy 2011; 9: 217-223.

17 Arribas J, Bernardino J, Hill A et al. Effects of switching to PI monotherapy on measures of lipoatrophy: meta-analysis of six randomized HIV clinical trials. Eleventh International Congress on Drug Therapy in HIV Infection. November 2012 [Abstract P287].

18 Karlstrom 0, Josephson F, Sonnerborg A. Early virologic rebound in a pilot trial of ritonavir-boosted atazanavir as maintenance monotherapy. J Acquir Immune Defic Syndr 2007; 44: 417-422.

19 Vernazza P, Daneel S, Schiffer V et al. Risk of CNS compartment failure on PI monotherapy (ATARITMO study). XVI International AIDS Conference. August 2006. Toronto, Canada [Abstract WEPE0073].

20 Bunupuradah T, Chetchotisakd P, Ananworanich J et al. A randomized comparison of second-line lopinavir/ritonavir monotherapy versus tenofovir/lamivudine/lopinavir/ ritonavir in patients failing NNRTI regimens: the HIV STAR study. Antivir Ther 2012; 17: 1351-1361.

21 Gilks CF, Walker AS, Dunn DT et al. Lopinavir/ritonavir monotherapy after 24 weeks of second-line antiretroviral therapy in Africa: a randomized controlled trial (SARA). Antivir Ther 2012; 17: 1363-1373.

22 Paton NI, Kityo C, Hoppe A et al. Assessment of second-line antiretroviral regimens for HIV therapy in Africa. $N$ Engl $J$ Med 2014; 371: 234-247.

\subsubsection{Treatment with one boosted protease inhibitor and} one NRTI

6.3.4.1 Recommendation

- We suggest that a boosted PI plus lamivudine as an alternative to three-drug ART in individuals with viral suppression (2A)

6.3.4.2 Rationale

Two studies have compared the use of boosted atazanavir or lopinavir with lamivudine versus a conventional threedrug regimen in patients with a suppressed viral load [1,2]. In both studies, the efficacy of dual therapy was non-inferior to conventional therapy after 48 weeks of follow up. In patients who have a suppressed viral load on a conventional regimen, but who have drug toxicity associated with abacavir or tenofovir-DF, dual therapy is thus a reasonable option. This approach has not been compared formally with protease inhibitor monotherapy, however.

\subsubsection{References}

1 Arribas JR, Girard PM, Landman R et al. Dual treatment with lopinavir-ritonavir plus lamivudine versus triple treatment with lopinavir-ritonavir plus lamivudine or emtricitabine and a second nucleos(t)ide reverse transcriptase inhibitor for maintenance of HIV-1 viral suppression (OLE): a randomised, open-label, non-inferiority trial. Lancet Infect Dis 2015; 15: 785-792.

2 Perez-Molina JA, Rubio R, Rivero A et al. Dual treatment with atazanavir-ritonavir plus lamivudine versus triple treatment with atazanavir-ritonavir plus two nucleos(t)ides in virologically stable patients with HIV-1 (SALT): 48 week results from a randomised, open-label, non-inferiority trial. Lancet Infect Dis 2015; 15: 775-784.

\subsection{Stopping therapy}

\subsubsection{Recommendation}

- We recommend against treatment interruption or intermittent therapy in individuals stable on a virally suppressive ART regimen (1A).

\subsubsection{Auditable outcomes}

- Proportion of individuals not on ART having previously been on ART.

- Documentation of reasons for stopping in those who stopped.

\subsubsection{Rationale}

Several randomised controlled trials have investigated the efficacy of CD4 cell count-guided intermittent therapy as a potential strategy to reduce long-term risk of drug toxicity and drug resistance [1-4]. In the largest of these, subjects were randomly allocated to either CD4 cell count-guided intermittent therapy (stopping ART once CD4 cell count $>350$ cells $/ \mu \mathrm{L}$, restarting when CD4 cell count falls to 250 cells $/ \mu \mathrm{L}$ ) compared with a continuous ART [4]. The trial showed intermittent therapy was associated with a significantly higher rate of opportunistic disease and all-cause mortality and a higher rate of major cardiovascular, renal or hepatic disease. The effect was seen at all CD4 cell count levels. The study showed for the first time that continuous ART with virological suppression is associated with a reduction in the risk of nonAIDS co-morbidities and all-cause mortality as well as HIV disease progression. For this reason, treatment interruption or intermittent therapy is not recommended.

Once ART has been started in a patient with HIV infection, it should be continued. An interruption of 1-2 days can usually be managed and is unlikely to be associated with adverse outcomes. Longer or frequent interruptions of ART should only be considered in exceptional circumstances. These may include:

- Severe drug toxicity (e.g. hepatotoxicity).

- Severe psychological distress. 
Guidance on pharmacokinetic considerations when stopping ART is contained in Section 6.2.3.

\subsubsection{References}

1 Ananworanich J, Gayet-Ageron A, Le Braz M et al. CD4guided scheduled treatment interruptions compared with continuous therapy for patients infected with HIV-1: results of the Staccato randomised trial. Lancet 2006; 368: 459465.

2 Cardiello PG, Hassink E, Ananworanich J et al. A prospective, randomized trial of structured treatment interruption for patients with chronic HIV type 1 infection. Clin Infect Dis 2005; 40: 594-600.

3 Danel C, Moh R, Minga A et al. CD4-guided structured antiretroviral treatment interruption strategy in HIV-infected adults in west Africa (Trivacan ANRS 1269 trial): a randomised trial. Lancet 2006; 367: 1981-1989.

4 El-Sadr WM, Lundgren J, Neaton JD et al. CD4 + countguided interruption of antiretroviral treatment. $N$ Engl $J$ Med 2006; 355: 2283-2296. 


\subsection{Managing virological failure}

\subsection{Introduction}

For detailed guidance on HIV viral load (VL), resistance and genotypic tropism testing, the reader should consult BHIVA guidelines for the routine investigation and monitoring of adult HIV-1-infected individuals [1] (www.bhiva.org/Monitoring.aspx).

The following recommendations concern the management of PLWH experiencing virological failure on ART. Populations at the time of virological failure will include those with no or limited HIV drug resistance, those with more extensive resistance or historical virological failure

Box 7.1 Best practice for the management of individuals with suspected or confirmed virological failure

- Factors affecting adherence and drug exposure, including tolerability/toxicity issues, drug-drug interactions/food interactions, ARV potency, significant renal/liver disease and mental health/drug dependency problems are evaluated.

- Resistance testing is performed while on failing therapy or within 2-4 weeks of discontinuation.

- Past ART and resistance tests are reviewed for archived mutations.

- Tropism testing is performed if maraviroc is being considered.

- Intensification with a single additional active ARV is not recommended.

- Once virological failure is confirmed and preferably after a resistance-test result is available, the regimen is changed as soon as possible to avoid accumulation of resistance mutations.

The choice of the new ART regimen will primarily depend on the results of resistance testing, prior treatment history and the individual's preference. Additional considerations include the results of tropism and HLA-B*5701 testing, drug-drug interactions/food interactions, co-morbidities and future therapy options. The goal of the new combination is to reestablish a VL $<50$ copies/mL. on NRTIs, NNRTIs, and/or PIs and those with limited treatment options, including failure of an integrase inhibitor or X4 tropic virus. For the assessment and evaluation of evidence, priority questions were agreed and outcomes were ranked as critical, important and not important by members of the writing group. For individuals with no or limited HIV drug resistance the following were ranked as critical outcomes: viral suppression to $<50$ copies $/ \mathrm{mL}$ at 48 weeks, development of resistance, and discontinuation for clinical and laboratory adverse events. For individuals with three-class failure/few therapeutic options: clinical progression, median CD4 cell count change at 48 weeks, and development of new resistance were ranked as critical outcomes. Treatments were compared where data were available and differences in outcomes assessed. For this update of the guidelines, the benefit of including NRTIs in the context of virological failure/resistance was examined. Details of the search strategy and literature review are contained in Appendix 2.

In the UK, the virological failure rate on first-line NNRTI-based regimens is $18 \%$ with approximately $4 \%$ failing in the first year [2]. The options for switch depend on the most recent and past ARV treatments as well as current and archived resistance results. As baseline genotypic testing of reverse transcriptase and protease (not integrase at the time of writing) is now performed routinely and is recommended practice, detection of resistance at virological failure is rarely a result of transmitted drug resistance and failure to adapt first-line treatment [3,4].

The general principles for the management of individuals experiencing virological failure are outlined in Boxes 7.1 and 7.2 as GPPs. Details of typical patterns of HIV drug resistance found in individuals with a history of or presenting with virological failure are outlined in Box 7.3.

\subsubsection{Summary of auditable measures}

- Record in medical notes of resistance result at baseline (HIV diagnosis) or at ART initiation (if former not available) and at first VL $>200$ copies $/ \mathrm{mL}$ after prior virological suppression (or less if genotyping successful) and/or before switch.

- Record in medical notes of adherence assessment and tolerability/toxicity to ART in individuals experiencing virological failure or repeated viral blips. 
Box 7.2 Best practice for the management of individuals with three-class virological failure

- In individuals with ongoing viraemia and with few options to construct a fully suppressive regimen, referral for specialist advice and/or discussion in a multidisciplinary team 'virtual' clinic is imperative.

- In those with significant resistance, include at least two and preferably three fully active agents with at least one active $\mathrm{PI} / \mathrm{r}$ (preferably darunavir/r) and one agent with a novel mechanism of action (preferably integrase inhibitor, CCR5 antagonist or fusion inhibitor).

- Treatment interruption is not recommended.

Box 7.3 Typical resistance patterns on virological failure

- No resistance (wild-type virus)

- Lamivudine/emtricitabine resistance (M184V/I) following any first-line therapy, including tenofovirDF/emtricitabine or abacavir/lamivudine.

- NNRTI resistance (e.g. K103N, Y181C/I/V or E138K) and/or lamivudine/emtricitabine resistance (following first-line therapy with an NNRTI-based regimen, including tenofovir-DF/emtricitabine or abacavir/ lamivudine).

- INI resistance (e.g. Y143C/R, Q148R/H or N155H) and/or lamivudine/emtricitabine resistance (following first-line therapy with raltegravir or elvitegravir-based regimens, including tenofovir-DF/ emtricitabine or abacavir/lamivudine).

- Extended reverse transcriptase resistance (e.g. K65R/L74V or thymidine analogue mutations) (following suboptimal regimens/individuals with more extensive NRTI-based drug history associated with virological failure).

- Three-class resistance (usually NRTI, NNRTI and PI) (following multiple failing regimens).

- Limited or no therapeutic options (following multiple failing regimens, including integrase and R5 inhibitors).

- Proportion of individuals experiencing virological failure on current ART regimen.

- Proportion of individuals experiencing virological failure switched to a new suppressive regimen within 6 months.
- Proportion of individuals on ART with previously documented HIV drug resistance with VL <50 copies/mL.

- Record of individuals with three-class virological failure with or without three-class resistance referred/discussed in multidisciplinary team with expert advice.

\subsection{Blips, low-level viraemia and virological failure}

Definitions (in the context of continued ART without changes):

- Virological suppression: achieving and maintaining a VL level <50 copies/mL.

- Virological failure: incomplete virological response after commencing treatment or evidence of confirmed virological rebound to $>200$ copies $/ \mathrm{mL}$.

- Incomplete virological response: two consecutive VL $>200$ copies/mL after 24 weeks without ever achieving VL $<50$ copies $/ \mathrm{mL}$. Consideration of the baseline VL and regimen should be made as some regimens will take longer than others to suppress HIV RNA levels. In individuals with a high baseline viral load (e.g. $>100,000$ copies $/ \mathrm{mL}$ ) it may take longer for viral load to fall below the limit of detection; in contrast, individuals treated with an integrase inhibitor are more likely to experience more rapid reduction in viral load.

- Virological rebound: failure to maintain a VL below the limit of detection (ordinarily $<40-50$ copies $/ \mathrm{mL}$ ) on two or more consecutive occasions.

- Low-level viraemia: a persistent VL between 50 and 200 copies/mL.

- Virological blip: after virological suppression, a single VL between 50 and 200 copies/mL followed by an undetectable result.

\subsubsection{Recommendations}

In individuals on ART:

- A single VL 50-200 copies/mL preceded and followed by an undetectable VL is usually not a cause for clinical concern (GPP). It should necessitate clinical vigilance, adherence reinforcement, check for possible interactions, and repeat testing within 2-6 weeks depending on ARV regimen.

- We recommend that a single VL $>200$ copies $/ \mathrm{mL}$ is investigated further, including a rapid re-test +/- genotypic resistance test, as it may be indicative of virological failure (1C).

- We recommend that in the context of low-level viraemia or repeated viral blips, resistance testing be attempted (1D). 


\subsubsection{Rationale}

\subsubsection{Blips}

Optimal HIV control is ordinarily reflected by complete virological suppression with an undetectable VL. A virological blip is variably defined but for the purposes of these guidelines the definition that has been adopted is a detectable VL between 50 and 200 copies $/ \mathrm{mL}$, which is preceded by and followed by an undetectable result without any change of therapy. Blips are frequent, tend to cluster between 50 and 200 copies/mL, (median magnitude 79 copies/mL in one study with range between 51201 copies $/ \mathrm{mL}$ ) and, when real and not due to laboratory variability, are short-lived (median 2.5 days, range 211.5 days) [5-7]. Many individuals have at least one blip at some time [8] and most studies have found no relationship between isolated blips and adverse outcomes such as virological failure or emergent resistance $[5,9,10]$. However some studies have reported an association between blips and future virological failure $[6,11]$.

There is correlation between level of first detectable viral load and subsequent virological rebound [8,12]. One retrospective study of over 3000 individuals found virological failure (defined as consecutive HIV-1 VL $>50$ copies/mL measured at least 30 days apart, or any VL $>1000$ copies $/ \mathrm{mL}$ ) in $26 \% ; 14 \%$ of rebounds were preceded by transient HIV-1 VL of 50-999 copies/mL but critically, only transient HIV VL $>500$ copies/mL correlated with rebound in multivariable analyses [12]. This concurs with other studies (see Section 7.2.2.2).

VL assay variation and laboratory processing artefacts account for many blips (i.e. no 'true' increase in viral replication), which partly explains why blips do not appear to compromise long-term outcomes [9,13,14]. Most individuals with short-lived increases in HIV VL to $<200$ copies/mL can be reassured that such events are relatively common and unlikely to presage failure. However, those with sustained low-level increases in VL (see 7.2.2.2) run a higher risk of virological failure. In keeping with the DHSS guidance [15], these guidelines [16] define virological failure as a confirmed viral load $>200$ copies/ $\mathrm{mL}$, a threshold that eliminates most cases of viral load blips.

A detectable viral load should prompt a review of adherence (and reiteration of the importance of full adherence), as well as looking for any tolerability/toxicity issues, drug-drug interactions/food interactions, and evidence of archived resistance. A VL result of 50-200 copies/mL preceded and followed by an undetectable VL should not be a cause of clinical concern. In the context of repeated blips or persistent low-level viraemia, genotypic resistance testing is recommended [11,17].

7.2.2.2 Low-level viraemia
Low-level viraemia (LLV) is observed in up to $8 \%$ of individuals [18] and, when compared to viral suppression to $<50$ copies/mL, is associated with an increased risk of virological failure and resistance $[6,19,20]$. The likelihood of re-suppression after LLV is greater for lower magnitudes of viraemia [21]. Indeed it is uncertain whether LLV $<200$ copies/mL always confers independent risks as viraemia at this level may on occasions reflect assay variation. LLV is associated with resistance (37\% in one study [20]) that may be associated with LLV magnitude; in one analysis, maximum VL was higher in those who developed resistance (368 vs. 143 copies $/ \mathrm{mL}, P=0.008$ ). In cohort studies [19] and clinical trials [20], individuals on PI/r-based ART are more likely to experience detectable viraemia than those on NNRTI. Many individuals with LLV have low or undetectable plasma drug levels on untimed samples underscoring the importance of assessing adherence [22]; we do not however recommend routine therapeutic drug monitoring in this context (see Section 6.2.2). LLV is also associated with immune activation [10]. Low-level antigenic exposure differentially affects $\mathrm{T}$ cell activation and HIV-specific T cell response.

Resistance testing should be considered, where feasible, in all cases of LLV (viraemia between 50 and 200 copies/ $\mathrm{mL}$ ) on treatment. Where resistance is detected, regimens should be modified appropriately. In the absence of clear data, the writing group believes persistent LLV on a lowgenetic barrier regimen (including NNRTI-based or INIbased therapy), even in the absence of detectable resistance, warrants prompt regimen change [23,24].

Further evaluation should follow as for that set out in Box 7.1.

Increasingly, viral load assays have quantification cutoffs lower than 50 copies/mL. Thus, individuals may have persistent viraemia $>20$ or $>40$ copies/mL but $<50$ copies/ $\mathrm{mL}$, depending on the assay used. Rates of this "very lowlevel' viraemia (VLLV) are as yet unclear. Several studies have evaluated the risk of virological rebound $>50$ copies/mL in individuals with detectable viraemia below 50 copies/mL. Results are conflicting [25-27]. In one study, subjects were stratified based on the Abbott RealTime Assay into VL 40-49 copies/mL, <40 copies/mL with RNA detected, and $<40$ copies/mL with no RNA detected [26]. They found that compared to individuals with VL $<40$ copies/mL and no detected RNA, having viraemia of $40-49$ copies $/ \mathrm{mL}$ increased the risk of rebound to $>50$ copies $/ \mathrm{mL}$ by 4.67 -fold while having detectable RNA at $<40$ copies/mL increased the risk by 1.97 -fold. The risk of rebound to $>400$ copies $/ \mathrm{mL}$ was increased by 6.91-fold and 2.88-fold, respectively. Other studies have reported increased risk of rebound to $>50,>200,>400$ copies/mL but importantly, not $>1000$ copies $/ \mathrm{mL}$ or 
higher [28]. The majority of the rebounds $>200$ copies $/ \mathrm{mL}$ were blips and resistance rarely emerged [28], making the significance of these events unclear.

In the absence of clear data, the writing group believes that, having assessed factors outlined in Box 7.1, no treatment modification is required for individuals with detectable viraemia below 50 copies $/ \mathrm{mL}$.

7.2.2.3 Virological failure

In the UK, among drug-experienced individuals who experience virological failure approximately 70\% have no major resistance mutations on genotypic resistance testing [29]. Confirmation of virological failure at any stage should lead to the practice set out in Box 7.1.

\subsection{Individuals with no or limited drug resistance}

\subsubsection{Recommendations}

- We recommend for individuals experiencing virological failure on first-line ART with wild-type virus at baseline and without emergent resistance mutations at failure, switch to a $\mathrm{PI} / \mathrm{r}$-based combination ART regimen is the preferred option (1C).

- We recommend individuals experiencing virological failure on first-line ART with wild-type virus at baseline and limited emergent resistance mutations (including two-class NRTI/NNRTI) at failure, switch to a new $\mathrm{PI} / \mathrm{r}$-based regimen with the addition of at least one, preferably two, active drugs (1C).

- We recommend individuals experiencing virological failure on first-line PI/r plus two-NRTI-based regimens, with limited major protease mutations, switch to a new active $\mathrm{PI} / \mathrm{r}$ with the addition of at least one, preferably two, active agents of which one has a novel mechanism of action (1C).

- We recommend against switching a $\mathrm{PI} / \mathrm{r}$ to an INI or NNRTI as the third agent in individuals with historical or existing reverse transcriptase mutations associated with NRTI resistance or past virological failure on NRTIs (1B).

\subsubsection{Rationale}

7.3.2.1 First-line treatment failure with no resistance Seventy per cent of individuals have wild-type virus despite failure of therapy [30-36]. Failure is usually attributable to poor treatment adherence with drug levels that are both insufficient to maintain VL suppression and inadequate to select out viral mutations associated with drug resistance detectable on standard tests. Factors affecting adherence such as tolerability/toxicity issues, regimen convenience, drug-food interactions and mental health/drug dependency problems should be fully evaluated and where possible corrected before initiation of the new regimen. Additional adherence support should be considered and careful discussion with the individual take place. Therapeutic drug monitoring may be of benefit in individuals to confirm low/absent therapeutic drug levels and to enable targeted discussion.

A priority question to address was whether individuals whose NNRTI-based ART had failed without detectable resistance should receive a PI/r-based regimen.

The absence of detectable resistance mutations does not exclude the presence of mutations in minor virus populations, especially with the NNRTIs [9,10,37]. This may increase the likelihood of subsequent failure if the same first-line drugs, or drugs in the same class, are prescribed [38,39]. Nevertheless, testing for minority resistance is a specialist test and expert interpretation by a virologist is essential. There is no indication for routine minority-species testing for individuals with wild-type virus and failed therapy.

We recommend that, following the development of virological failure, or persistent low-level viraemia, on either an NNRTI or INI-based ART regimen with two NRTIs and when no resistance mutations are detected, a switch to a PI/r-based regimen is optimal. This should lead to virological suppression, and is least likely to select emergent resistance. Restarting the previous failing regimen is an alternative option, especially where poor adherence has been identified as the likely cause and has been addressed. However, the subject should be monitored carefully and repeat VL performed after approximately 4 weeks. If there is inadequate virological response, resistance testing should be performed to detect any archived resistance. Switching to another NNRTI-, an INI- or maraviroc- (where CCR5 tropism has been confirmed) based ART regimen is the final option but has to be individualised, including history of virological failure, and whether further switches in the combination are occurring. In deciding which option, knowledge as to the likely cause of virological failure especially the details of poor adherence are important. In an NNRTI/2NRTI regimen, when all three agents have been stopped, the chances of NNRTI resistance are $12-16 \%$ depending on whether there is a simultaneous or staggered interruption [40,41]

7.3.2.2 First-line treatment failure with non-nucleoside reverse transcriptase inhibitor resistance

Up to two-thirds of PLWH with virological failure on an NNRTI/2NRTI ART combination harbour viruses with NNRTI and half NRTI mutations at 48 weeks [33-36,42]: with increasing time, there will be accumulation of resistance mutations that may compromise second-line regimens [43]. The finding of associated NRTI resistance is 
more frequent in those on a thymidine analogue (TA) backbone than on a non-TA one. Although potential options for second-line therapy after failure on an NNRTI-containing regimen include an integrase inhibitor (raltegravir, elvitegravir or dolutegravir), etravirine or maraviroc as the third agent, evidence supports the use of a PI/r. A switch to any PI/r-based regimen should lead to virological suppression and is unlikely to lead to further emergent resistance and should be considered whenever possible. Where NRTI resistance has been documented or likely, the addition of new active NRTIs or another $\mathrm{ARV}(\mathrm{s})$ should be considered in combination with a boosted PI. The exception to this is when M184V is present alone, when recycling of NRTIs may be feasible. Combining raltegravir with a boosted-PI has been found to be as efficacious as a boosted $\mathrm{PI} / \mathrm{r}$ regimen with at least two new or recycled NRTIs [44-46].

There are no direct comparisons of the boosted PIs in second-line treatment after first-line failure on an NNRTI-based regimen and choice should be individualised. Sequencing from an efavirenz- or nevirapinebased regimen to etravirine is not recommended [47] unless switching to a new combination including a boosted PI. Switching to an INI (raltegravir, elvitegravir or dolutegravir) or maraviroc with two active NRTIs is an option but is also not recommended if there are historical or existingreverse transcriptase mutations/previous NRTI virological failure [48].

7.3.2.3 First-line treatment failure on a ritonavir-boosted protease inhibitor-based two nucleoside reverse transcriptase inhibitor regimen with or without protease inhibitor resistance

Less than $1 \%$ of individuals with virological failure harbour viruses with primary PI mutations and 10-20\% NRTI mutations at 48 weeks, with 75\% having wild-type virus $[30,33-35,49,50]$. For those whose regimens fail with limited or no resistance and where adherence is a concern, remaining on the same regimen may be a reasonable approach but with close monitoring and adherence support. However, the individual should be monitored carefully and repeat VL performed after approximately 4 weeks. If there is inadequate virological response, resistance testing should be performed to detect any additional archived resistance. There are currently limited data regarding the efficacy of switching to another PI/r, NNRTI, INI or maraviroc-based regimen and again the decision should individualised. However, switching to an INI, maraviroc or an NNRTI for a person with historical or existing reverse transcriptase mutations is not recommended because of an increased risk of virological failure and further emergence of resistance [48]. By contrast, because of the high genetic barrier of $\mathrm{PI} / \mathrm{r}$, sequencing to a regimen that includes a new $\mathrm{PI} / \mathrm{r}$ is unlikely to lead to further emergent resistance and is recommended. Where $\mathrm{PI} / \mathrm{r}$ mutations exist, darunavir/ $\mathrm{r}$ is the preferred agent (unless resistance is likely) and inclusion of an INI, etravirine or maraviroc (if R5 tropic virus) as one of the additional drugs should be considered. Where darunavir/r is not suitable, depending on susceptibility, alternative PIs such as tipranavir/r and lopinavir/r may be considered.

7.3.2.4 First-line treatment failure with integrase inhibitor-based resistance

In studies of naïve subjects developing virological failure on raltegravir or elvitegravir regimens, up to one-half have been found to harbour viruses with primary integrase mutations and 25\% NRTI mutations at 48 weeks: approximately half have wild-type virus [32,42,49,51]. By contrast, no resistance has been seen in studies in treatment-naive individuals with dolutegravir/2NRTI-based regimens [52-54]. Again, there are no data supporting a switch to $\mathrm{PI} / \mathrm{r}$, NNRTI or maraviroc but sequencing to a new regimen that includes a $\mathrm{PI} / \mathrm{r}$ is unlikely to lead to further emergent resistance and is recommended. Similarly, although data from the VIKING-3 study in individuals with pre-existing integrase mutations after failure on raltegravir or elvitegravir in the context of three-class resistance and with optimisation of the background regimen has shown over half achieve a VL $<50$ copies/mL [55], there are no data to support sequencing to dolutegravir after first-line failure. Switching to an NNRTI or maraviroc with two active NRTIs is an option but is also not recommended in a person with historical or existing reverse transcriptase mutations or previous NRTI virological failure. Individuals experiencing virological failure on raltegravir or elvitegravir should switch to a new regimen as soon as possible to reduce the risk of accumulating resistance mutations that may affect susceptibility to dolutegravir where success of response has been linked to the profile and number of resistance mutations.

7.4 Individuals with multiple class virological failure with or without extensive drug resistance

\subsubsection{Recommendations}

- We recommend individuals with persistent viraemia and with limited options to construct a fully suppressive regimen are discussed/referred for expert advice (or through virtual clinic referral) (GPP).

- We recommend individuals with extensive drug resistance are switched to a new ART regimen containing at least two and preferably three fully active agents with at least one active $\mathrm{PI} / \mathrm{r}$ such as darunavir/r and one agent with a novel mechanism (an INI, maraviroc 
or enfuvirtide) with etravirine an option based on viral susceptibility (1C).

- We recommend individuals with extensive drug resistance including reduced darunavir susceptibility receive dolutegravir as the INI (1C).

- We suggest that consideration on an individual basis should be given to whether inclusion of NRTIs with reduced activity on genotypic testing will provide additional antiviral activity if the regimen includes three fully active drugs including a boosted PI (2C).

- We recommend all individuals receive intensive adherence support at the start and at regular intervals to support them on their new ART combination (GPP).

\subsubsection{Rationale}

The risk of extended three-class resistance, defined as the absence of any fully active NRTI, NNRTI, or PI, was only 2\% among drug-experienced individuals in Western Europe in 2008 [56]. Ongoing treatment non-adherence drives further virological failure and addressing this and supporting strategies to improve adherence are important considerations before the introduction of any new regimen. Until the last 5 years, limited treatment options have been available for people with HIV who have had virological failure with the three original classes of HIV ARV drugs for which many have developed triple-class resistance. Most of these individuals have received prior suboptimal ARV treatment, often from the pre-ART era, or have adhered poorly to multiple regimens and have accumulated resistance. However, with the introduction of second-generation inhibitors of reverse transcriptase and protease that have enhanced activity against resistant virus as well as agents active through novel sites of action, the potential for virological control akin to that achieved in a treatment-naïve person has now become a probability $[57,58]$.

Consequent to more active ARVs and improved strategies of management, there has been substantial improvement in the proportion of people who had virological response after triple-class virological failure between 2000 and 2009 [59]. However, despite improvements in treatments, viral load cannot be suppressed in some individuals. In most, this is a result of poor adherence but some individuals do have extended drug resistance with minimal treatment options and achieving viral suppression becomes increasingly difficult.

The drugs currently being used in triple-class failure are the boosted PIs (predominantly twice-daily darunavir/ $\mathrm{r}$ but also on occasions tipranavir/r), the INIs raltegravir and dolutegravir, the CCR5 chemokine receptor antagonist maraviroc, the NNRTI etravirine, and the fusion inhibitor enfuvirtide. The available data for darunavir/r, tipranavir/r, raltegravir, elvitegravir, dolutegravir, etravirine and enfuvirtide show that they are most effective when used with other active drugs to which the virus is susceptible based on resistance testing and antiviral experience [60-68]. When used as the only effective agent, the likelihood of achieving virological suppression is significantly reduced and the development of emergent resistance to the drug greater, and a future opportunity for constructing an effective regimen is often lost.

In a meta-analysis of 10 trials (excluding dolutegravir) of subjects with triple-class virological failure and virological resistance where the study drug was added to optimised background therapy and compared with placebo, associations were demonstrated with increased virological suppression (pooled OR 2.97) and larger CD4 cell count increases for the active agent [69]. Optimised background therapy genotypic sensitivity scores (GSSs) were also associated with larger differences in virological suppression and CD4 cell count increase between the two groups. In a further non-inferiority study, elvitegravir was found to be non-inferior to raltegravir when accompanied by a boosted PI and a third agent [61].

A non-inferiority trial comparing dolutegravir with raltegravir as the comparator examined those with tripleclass experience but who were naïve to integrase inhibitors and had at least two-class resistance and at least one fully active drug as optimised background therapy [70]. Overall, once-daily dolutegravir was superior to raltegravir at 48 weeks in achieving a VL $<50$ copies $/ \mathrm{mL}$. However, there was no benefit in individuals who had not received darunavir/r or had no primary darunavir mutations.

This supports the use of at least two and preferably three of these agents in the new regimen and with this strategy, the goal of an undetectable VL is achievable in most adherent individuals with multi-regimen failure.

A priority question addressed in this group was around the net contribution of recycling NRTIs in the context of virological failure and existing or potential reverse transcriptase mutations. In two studies examining individuals previously naïve to ART for whom an NNRTI/2NRTI regimen subsequently failed [44,45], a boosted PI/r regimen with at least two new or recycled NRTIs was no less efficacious than an NRTI-sparing regimen combining raltegravir with a boosted-PI. Even in the presence of limited or no predicted activity on the basis of genotypic assay, NRTIs retained substantial virological activity equivalent to that of raltegravir without evidence of increased toxicity and therefore may allow the introduction of drugs known to be active to be deferred. However, NRTI inclusion was demonstrated to achieve improved virological control over PI/r monotherapy out to 96 weeks [45]. 
Once virological suppression has been achieved, the advantage of retaining NRTIs where partial or complete resistance is demonstrated is uncertain. A small randomised open study of 90 virologically suppressed individuals evaluated the safety of withdrawing NRTIs compared to a control arm of maintaining them in the context of partial NRTI activity and the presence of at least two fully active remaining drugs in the regimen. No significant difference in virological failure between the arms was observed out to 48 weeks although there were three cases of virological failure in the simplification arm and none in the NRTI control arm [71].

A fourth study examined individuals who had tripleclass failure and/or resistance when randomisation to the new regimen was based on treatment history, tropism testing and resistance profiles including a choice of NRTIs [72]. Following randomisation, the subjects then received the chosen regimen with or without the NRTIs. The results demonstrated omitting NRTIs was non-inferior to their inclusion. Of note, subjects in this study received an average of three active drugs and therefore the lack of NRTI benefit is not altogether surprising.

A further uncertainty has been whether maintaining lamivudine/emtricitabine provides clinical benefit through the replication deficit provided by the M184V mutation combined with the residual antiviral activity of lamivudine/emtricitabine [73,74]. Studies using lamivudine monotherapy for individuals developing therapy failure have shown that those harbouring M184V who continue on lamivudine maintain lower VLs, have smaller declines in CD4 cell counts, and rarely develop new reverse transcriptase mutations [75-77]. In addition, the presence of $\mathrm{M} 184 \mathrm{~V}$ mutation enhances in vitro susceptibility to tenofovir-DF and this translates into a significant HIV RNA response in clinical trials of tenofovir-DF intensification $[78,79]$. Insufficient data exists to guide recommendations as to whether there are clinical benefits of trying to maintain M184V by continuing lamivudine/ emtricitabine when switching to new combination ART. It is the belief of the writing group that any decision should be individualised.

For those drugs with a novel mode of action (integrase and fusion inhibitors, and CCR5 antagonists), the absence of previous exposure indicates susceptibility, although maraviroc is only active against CCR5-tropic virus. For darunavir, tipranavir and etravirine, the number and type of mutations inform the degree to which these drugs are active [80-82]. The potential for drug-drug interactions is also important. Etravirine can be paired with darunavir/r (but not tipranavir/r or dolutegravir) and maraviroc dosing is variable depending on the other drugs in the new regimen; however, raltegravir and enfuvirtide require no alteration.

Some individuals can have a successfully suppressive fully active three-drug regimen constructed without a PI/ $r$ [83]. Nevertheless, where feasible, a PI/r such as darunavir/r should be included because of its protective effect on emergent resistance to the other drugs in the regimen. Darunavir/r can be given as $800 \mathrm{mg} / 100 \mathrm{mg}$ once daily in treatment-experienced individuals without darunavir resistance-associated mutations [84]. Enfuvirtide is an option despite the inconvenience of subcutaneous injection and injection-site reactions. With the availability of the newer agents, dual PI/r are not recommended [85].

The same principles regarding reviewing adherence, tolerability/toxicity issues, drug-drug interactions/food interactions, and mental health/drug dependency problems apply. Additional adherence support is important in these individuals as the reason triple-class failure has occurred often relates to past poor adherence. Additionally, the pill burden is increased and careful discussion should take place.

7.5 Individuals with limited or no therapeutic options when a fully viral suppressive regimen cannot be constructed

\subsubsection{Recommendations}

- We recommend accessing newer agents through research trials, expanded access and named individual programmes (GPP).

- We suggest that consideration, on an individual basis, should be given to whether inclusion of NRTIs with reduced activity on genotypic testing will provide additional antiviral activity - this may well be the case where it is difficult to construct a regimen with three fully active drugs including a boosted PI (see Section 7.4) (2C).

- We recommend against discontinuing or interrupting ART (1B).

- We recommend against adding a single, fully active ARV because of the risk of further resistance (1D).

- We recommend against the use of maraviroc to increase the CD4 cell count when there is evidence for $\mathrm{X} 4$ or dual tropic virus (1C).

- We recommend that in the context of triple-class failure and raltegravir/elvitegravir selected integrase resistance, twice-daily dolutegravir should be included as part of a new regimen where there is at least one fully active agent in the background regimen (1C). 


\subsubsection{Rationale}

This situation usually occurs following attempts to achieve virological suppression for individuals with triple-class failure with the newer agents, and often indicates adherence issues have not been addressed successfully or sequential addition of the newer agents has occurred without incomplete viral suppression and selection of resistance to the new drug.

There is evidence from cohort studies that continuing therapy, even in the presence of viraemia and the absence of CD4 T cell count increases, reduces the risk of disease progression $[86,87]$ whereas interruption may lead to a rapid fall in CD4 cell count and a rise in VL $[88,89]$. Other studies suggest continued immunological and clinical benefits if the HIV RNA level is maintained $<10,000-$ 20,000 copies/mL [90]. Hence, if the CD4 cell count is well maintained $(>200$ cells $/ \mu \mathrm{L})$, there is an argument to continue the failing regimen and not change treatment until investigational agents are available that can be put together with drugs, which may have only partial activity at best, to increase the likelihood of constructing virologically suppressive and durable regimen options. However, the potential benefit must be balanced with the ongoing risk of accumulating additional resistance mutations and the regimen should be maintained only for the shortest period possible [91,92].

In general, adding a single, fully active ARV to a failing regimen is not recommended because of the risk of rapid development of resistance. However, in individuals with a high likelihood of clinical progression (e.g. CD4 cell count $<100$ cells $/ \mu \mathrm{L}$ ) and limited drug options, adding a single drug may reduce the risk of immediate clinical progression, because even transient decreases in HIV RNA and/or transient increases in CD4 cell counts have been associated with clinical benefits [93].

Several studies and an early meta-analysis suggested that CCR5 receptor antagonists were associated with significant gains in CD4 cell counts even in the presence of $\mathrm{C}-\mathrm{X}-\mathrm{C}$ chemokine receptor type 4 tropic virus. However, a meta-analysis refuted this finding $(P=0.22)$ when comparing with other new drugs [69].

VIKING-3 [55] was a study of individuals who had received either raltegravir or elvitegravir and had integrase resistance with the majority having additional triple-class resistance, and where there was at least one fully active agent to use in the optimised background regimen. Dolutegravir $50 \mathrm{mg}$ twice daily was added to the failing regimen and by day 8 and at the time of switching to an optimised background regimen, the mean drop in VL was $\log _{10} 1.43$. By week $24,69 \%$ had achieved a VL $<50$ copies/mL. Response was associated with dolutegravir susceptibility and was most reduced in those with Q148 with at least two additional resistance mutations.

Where feasible, PLWH should be given the opportunity to enrol in research studies or expanded access programmes evaluating investigational new drugs. Drug availability is difficult to predict, but many second-and third-generation drugs and are in Phase IIb/III studies (e.g. maturation inhibitors [94]) and others with novel sites of action (e.g. CD4 receptor antagonists, etc.) are in earlier phases of development. Drugs developed for, and used in, other settings such as pegylated interferon that have been incidentally demonstrated to decrease VL should not be used without discussion with experienced HIV physicians in a multidisciplinary team because data are either too limited or contradictory.

\subsection{References}

1 Asboe D, Aitken C, Boffito M et al. British HIV Association guidelines for the routine investigation and monitoring of adult HIV-1-infected individuals 2011. HIV Med 2012; 13: $1-44$.

2 Waters L, Bansi L, Asboe D et al. Second-line protease inhibitor-based antiretroviral therapy after non-nucleoside reverse transcriptase inhibitor failure: the effect of a nucleoside backbone. Antivir Ther 2013; 18: 213-219.

3 Wittkop L, Gunthard HF, de Wolf F et al. Effect of transmitted drug resistance on virological and immunological response to initial combination antiretroviral therapy for HIV

(EuroCoord-CHAIN joint project): a European multicohort study. Lancet Infect Dis 2011; 11: 363-371.

4 Kuritzkes DR, Lalama CM, Ribaudo HJ et al. Preexisting resistance to nonnucleoside reverse-transcriptase inhibitors predicts virologic failure of an efavirenz-based regimen in treatment-naive HIV-1-infected subjects. J Infect Dis 2008; 197: 867-870.

5 Havlir DV, Bassett R, Levitan D et al. Prevalence and predictive value of intermittent viremia with combination hiv therapy. JAMA 2001; 286: 171-179.

6 Greub G, Cozzi-Lepri A, Ledergerber B et al. Intermittent and sustained low-level HIV viral rebound in patients receiving potent antiretroviral therapy. AIDS 2002; 16: 1967-1969.

7 Nettles RE, Kieffer TL. Update on HIV-1 viral load blips. Curr Opin HIV AIDS 2006; 1: 157-161.

8 Garcia-Gasco P, Maida I, Blanco F et al. Episodes of lowlevel viral rebound in HIV-infected patients on antiretroviral therapy: frequency, predictors and outcome. J Antimicrob Chemother 2008; 61: 699-704.

9 Nettles RE, Kieffer TL, Kwon P et al. Intermittent HIV-1 viremia (Blips) and drug resistance in patients receiving HAART. JAMA 2005; 293: 817-829. 
10 Karlsson AC, Younger SR, Martin JN et al. Immunologic and virologic evolution during periods of intermittent and persistent low-level viremia. AIDS 2004; 18: 981-989.

11 Easterbrook PJ, Ives N, Waters A et al. The natural history and clinical significance of intermittent viraemia in patients with initial viral suppression to $<400$ copies/ml. AIDS 2002; 16: 1521-1527.

12 Grennan JT, Loutfy MR, Su D et al. Magnitude of virologic blips is associated with a higher risk for virologic rebound in HIV-infected individuals: a recurrent events analysis. $J$ Infect Dis 2012; 205: 1230-1238.

13 Lima V, Harrigan R, Montaner JS. Increased reporting of detectable plasma HIV-1 RNA levels at the critical threshold of 50 copies per milliliter with the Taqman assay in comparison to the Amplicor assay. J Acquir Immune Defic Syndr 2009; 51: 3-6.

14 Stosor V, Palella FJ Jr, Berzins B et al. Transient viremia in HIV-infected patients and use of plasma preparation tubes. Clin Infect Dis 2005; 41: 1671-1674.

15 Ribaudo H, Lennox J, Currier J et al. Virologic failure endpoint definition in clinical trials: is using HIV-1 RNA threshold $<200$ copies/mL better than $<50$ copies/mL? An analysis of ACTG studies. 16th Conference on Retroviruses and Opportunistic Infections. March 2009. Montreal, Canada [Abstract 580].

16 DHSS. Guidelines for the use of antiretroviral agents in HIV-1 infected adults and adolescents. 2014. Available at: http://aidsinfo.nih.gov/guidelines (accessed September 2015).

17 Pozniak A, Gupta RK, Pillay D et al. Causes and consequences of incomplete HIV RNA suppression in clinical trials. HIV Clin Trials 2009; 10: 289-298.

18 Cohen C. Low-level viremia in HIV-1 infection: consequences and implications for switching to a new regimen. HIV Clin Trials 2009; 10: 116-124.

19 Geretti AM, Smith C, Haberl A et al. Determinants of virological failure after successful viral load suppression in first-line highly active antiretroviral therapy. Antivir Ther 2008; 13: 927-936.

20 Taiwo B, Gallien S, Aga E et al. Antiretroviral drug resistance in HIV-1-infected patients experiencing persistent low-level viremia during first-line therapy. $J$ Infect Dis 2011; 204: 515-520.

21 Staszewski S, Sabin C, Dauer B et al. Definition of loss of virological response in trials of antiretroviral drugs. AIDS 2003; 17: 1997-1998.

22 Alejandro G, Swenson L, Nohpal A et al. Untimed drug levels and resistance in patients experiencing low-level HIV viremia. Conference on Retroviruses and Opportunistic infections. February 2015. Seattle, WA, USA [Abstract 117].

23 Prosperi MC, Mackie N, Di Giambenedetto S et al. Detection of drug resistance mutations at low plasma HIV-1 RNA load in a European multicentre cohort study. J Antimicrob Chemother 2011; 66: 1886-1896.

24 Gallien S, Delaugerre C, Charreau I et al. Emerging integrase inhibitor resistance mutations in raltegravirtreated HIV-1-infected patients with low-level viremia. AIDS 2011; 25: 665-669.

25 Maggiolo F, Callegaro A, Cologni G et al. Ultrasensitive assessment of residual low-level HIV viremia in HAARTtreated patients and risk of virological failure. $J$ Acquir Immune Defic Syndr 2012; 60: 473-482.

26 Doyle T, Smith C, Vitiello P et al. Plasma HIV-1 RNA detection below 50 copies $/ \mathrm{ml}$ and risk of virologic rebound in patients receiving highly active antiretroviral therapy. Clin Infect Dis 2012; 54: 724-732.

27 Alvarez Estevez M, Chueca Porcuna N, Guillot Suay V et al. Quantification of viral loads lower than 50 copies per milliliter by use of the Cobas AmpliPrep/Cobas TaqMan HIV-1 test, version 2.0, can predict the likelihood of subsequent virological rebound to $>50$ copies per milliliter. J Clin Microbiol 2013; 51: 1555-1557.

28 Henrich TJ, Wood BR, Kuritzkes DR. Increased risk of virologic rebound in patients on antiviral therapy with a detectable HIV load $<48$ copies/mL. PLoS One 2012; 7: e50065.

29 Dolling D, Nelson M, Schwenk A et al. Rapid increase in the frequency of wild type HIV-1 drug resistance reports among ART-experienced patients in the UK. 20th Conference on Retroviruses and Opportunistic Infections. March 2013. Atlanta, GA, USA.

30 Ortiz R, Dejesus E, Khanlou H et al. Efficacy and safety of once-daily darunavir/ritonavir versus lopinavir/ritonavir in treatment-naive HIV-1-infected patients at week 48. AIDS 2008; 22: 1389-1397.

31 Molina JM, Andrade-Villanueva J, Echevarria J et al. Oncedaily atazanavir/ritonavir versus twice-daily lopinavir/ ritonavir, each in combination with tenofovir and emtricitabine, for management of antiretroviral-naive HIV1-infected patients: 48 week efficacy and safety results of the CASTLE study. Lancet 2008; 372: 646-655.

32 Lennox JL, DeJesus E, Lazzarin A et al. Safety and efficacy of raltegravir-based versus efavirenz-based combination therapy in treatment-naive patients with HIV-1 infection: a multicentre, double-blind randomised controlled trial. Lancet 2009; 374: 796-806.

33 Riddler SA, Haubrich R, DiRienzo AG et al. Class-sparing regimens for initial treatment of HIV-1 infection. $N$ Engl $J$ Med 2008; 358: 2095-2106.

34 Daar ES, Tierney C, Fischl MA et al. Atazanavir plus ritonavir or efavirenz as part of a 3-drug regimen for initial treatment of HIV-1. Ann Intern Med 2011; 154: 445-456.

35 Soriano V, Arasteh K, Migrone H et al. Nevirapine versus atazanavir/ritonavir, each combined with tenofovir disoproxil 
fumarate/emtricitabine, in antiretroviral-naive HIV-1 patients: the ARTEN Trial. Antivir Ther 2011; 16: 339-348.

36 Rimsky L, Vingerhoets J, Van Eygen V et al. Genotypic and phenotypic characterization of HIV-1 isolates obtained from patients on rilpivirine therapy experiencing virologic failure in the phase 3 ECHO and THRIVE studies: 48-week analysis. J Acquir Immune Defic Syndr 2012; 59: 39-46.

37 Roche Diagnostics GmbH M, Germany. COBAS Amplicor HIV-1 MONITOR Test, Version 1.5. June 2007.

38 Li JZ, Paredes R, Ribaudo HJ et al. Low-frequency HIV-1 drug resistance mutations and risk of NNRTI-based antiretroviral treatment failure: a systematic review and pooled analysis. JAMA 2011; 305: 1327-1335.

39 Paredes R, Lalama CM, Ribaudo HJ et al. Pre-existing minority drug-resistant HIV-1 variants, adherence, and risk of antiretroviral treatment failure. J Infect Dis 2010; 201: 662-671.

40 Geretti AM, Fox Z, Johnson JA et al. Sensitive assessment of the virologic outcomes of stopping and restarting nonnucleoside reverse transcriptase inhibitor-based antiretroviral therapy. PLoS One 2013; 8: e69266.

41 Cambiano V, Castro H, Chadwick D et al. Detection of NNRTI resistance mutations after interrupting NNRTI-based regimens. 21st Conference on Retroviruses and Opportunistic Infections. March 2014. Boston, MA, USA [Abstract 593].

42 Sax P, DeJesus E, Mills A et al. Co-formulated elvitegravir, cobicistat, emtricitabine, and tenofovir versus co-formulated efavirenz, emtricitabine, and tenofovir for initial treatment of HIV-1 infection: a randomised, double-blind, phase 3 trial, analysis of results after 48 weeks. Lancet 2012; 379: 2439-2448.

43 Cozzi-Lepri A, Paredes R, Phillips AN et al. The rate of accumulation of nonnucleoside reverse transcriptase inhibitor (NNRTI) resistance in patients kept on a virologically failing regimen containing an NNRTI*. HIV Med 2012; 13: 62-72.

44 Boyd MA, Kumarasamy N, Moore CL et al. Ritonavirboosted lopinavir plus nucleoside or nucleotide reverse transcriptase inhibitors versus ritonavir-boosted lopinavir plus raltegravir for treatment of HIV-1 infection in adults with virological failure of a standard first-line ART regimen (SECOND-LINE): a randomised, open-label, non-inferiority study. Lancet 2013; 381: 2091-2099.

45 Paton NI, Kityo C, Hoppe A et al. Assessment of second-line antiretroviral regimens for HIV therapy in Africa. $N$ Engl $J$ Med 2014; 371: 234-247.

46 Amin J, Boyd MA, Kumarasamy N et al. Raltegravir noninferior to nucleoside based regimens in second-line therapy with lopinavir/ritonavir over 96 weeks: a randomised open label study for the treatment of HIV-1 infection. PLoS One 2015; 10: e0118228.
47 Ruxrungtham K, Pedro RJ, Latiff GH et al. Impact of reverse transcriptase resistance on the efficacy of TMC125 (etravirine) with two nucleoside reverse transcriptase inhibitors in protease inhibitor-naive, nonnucleoside reverse transcriptase inhibitor-experienced patients: study TMC125C227. HIV Med 2008; 9: 883-896.

48 Eron JJ, Young B, Cooper DA et al. Switch to a raltegravirbased regimen versus continuation of a lopinavir-ritonavirbased regimen in stable HIV-infected patients with suppressed viraemia (SWITCHMRK 1 and 2): two multicentre, double-blind, randomised controlled trials. Lancet 2010; 375: 396-407.

49 Dejesus E, Rockstroh J, Henry K et al. Co-formulated elvitegravir, cobicistat, emtricitabine, and tenofovir disoproxil fumarate versus ritonavir-boosted atazanavir plus co-formulated emtricitabine and tenofovir disoproxil fumarate for initial treatment of HIV-1 infection: a randomised, double-blind, phase 3, non-inferiority trial. Lancet 2012; 379: 2429-2438.

50 Molina JM, Andrade-Villanueva J, Echevarria J et al. Oncedaily atazanavir/ritonavir compared with twice-daily lopinavir/ritonavir, each in combination with tenofovir and emtricitabine, for management of antiretroviral-naive HIV1-infected patients: 96-week efficacy and safety results of the CASTLE study. J Acquir Immune Defic Syndr 2010; 53: 323-332.

51 Eron JJ Jr, Rockstroh JK, Reynes J et al. Raltegravir once daily or twice daily in previously untreated patients with HIV-1: a randomised, active-controlled, phase 3 noninferiority trial. Lancet Infect Dis 2011; 11: 907-915.

52 Walmsley SL, Antela A, Clumeck N et al. Dolutegravir plus abacavir-lamivudine for the treatment of HIV-1 infection. $N$ Engl J Med 2013; 369: 1807-1818.

53 Clotet B, Feinberg J, van Lunzen J et al. Once-daily dolutegravir versus darunavir plus ritonavir in antiretroviral-naive adults with HIV-1 infection (FLAMING0): 48 week results from the randomised openlabel phase 3b study. Lancet 2014; 383: 2222-2231.

54 Raffı F, Rachlis A, Stellbrink HJ et al. Once-daily dolutegravir versus raltegravir in antiretroviral-naive adults with HIV-1 infection: 48 week results from the randomised, double-blind, non-inferiority SPRING-2 study. Lancet 2013; 381: 735-743.

55 Castagna A, Maggiolo F, Penco G et al. Dolutegravir in antiretroviral-experienced patients with raltegravir- and/or elvitegravir-resistant HIV-1: 24-week results of the phase III VIKING-3 study. J Infect Dis 2014; 210: 354-362.

56 De Luca A, Dunn D, Zazzi M et al. Declining prevalence of HIV-1 drug resistance in antiretroviral treatment-exposed individuals in Western Europe. J Infect Dis 2013; 207: 1216-1220. 
57 Yazdanpanah Y, Fagard C, Descamps D et al. High rate of virologic suppression with raltegravir plus etravirine and darunavir/ritonavir among treatment-experienced patients infected with multidrug-resistant HIV: results of the ANRS 139 TRIO trial. Clin Infect Dis 2009; 49: 1441-1449.

58 Wittkop L, Breilh D, Da Silva D et al. Virological and immunological response in HIV-1-infected patients with multiple treatment failures receiving raltegravir and optimized background therapy, ANRS $\mathrm{CO} 3$ Aquitaine Cohort. J Antimicrob Chemother 2009; 63: 1251-1255.

59 Castagliola D, Ledergerber B, Torti C et al. Trends in virological and clinical outcomes in individuals with HIV-1 infection and virological failure of drugs from three antiretroviral drug classes: a cohort study. Lancet Infect Dis 2012; 12: 119-127.

60 Hicks CB, Cahn P, Cooper DA et al. Durable efficacy of tipranavir-ritonavir in combination with an optimised background regimen of antiretroviral drugs for treatmentexperienced HIV-1-infected patients at 48 weeks in the Randomized Evaluation of Strategic Intervention in multidrug reSistant patients with Tipranavir (RESIST) studies: an analysis of combined data from two randomised open-label trials. Lancet 2006; 368: 466-475.

61 Molina JM, Lamarca A, Andrade-Villanueva J et al. Efficacy and safety of once daily elvitegravir versus twice daily raltegravir in treatment-experienced patients with HIV-1 receiving a ritonavir-boosted protease inhibitor: randomised, double-blind, phase 3, non-inferiority study. Lancet Infect Dis 2012; 12: 27-35.

62 Lalezari JP, Henry K, O'Hearn M et al. Enfuvirtide, an HIV1 fusion inhibitor, for drug-resistant HIV infection in North and South America. N Engl J Med 2003; 348: 2175-2185.

63 Lazzarin A, Clotet B, Cooper D et al. Efficacy of enfuvirtide in patients infected with drug-resistant HIV-1 in Europe and Australia. N Engl J Med 2003; 348: 2186-2195.

64 Katlama C, Haubrich R, Lalezari J et al. Efficacy and safety of etravirine in treatment-experienced, HIV-1 patients: pooled 48 week analysis of two randomized, controlled trials. AIDS 2009; 23: 2289-2300.

65 Gulick RM, Lalezari J, Goodrich J et al. Maraviroc for previously treated patients with R5 HIV-1 infection. $N$ Engl J Med 2008; 359: 1429-1441.

66 Grinsztejn B, Nguyen BY, Katlama C et al. Safety and efficacy of the HIV-1 integrase inhibitor raltegravir (MK0518) in treatment-experienced patients with multidrugresistant virus: a phase II randomised controlled trial. Lancet 2007; 369: 1261-1269.

67 Clotet B, Bellos N, Molina JM et al. Efficacy and safety of darunavir-ritonavir at week 48 in treatment-experienced patients with HIV-1 infection in POWER 1 and 2: a pooled subgroup analysis of data from two randomised trials. Lancet 2007; 369: 1169-1178.
68 Steigbigel RT, Cooper DA, Kumar PN et al. Raltegravir with optimized background therapy for resistant HIV-1 infection. N Engl J Med 2008; 359: 339-354.

69 Pichenot M, Deuffic-Burban S, Cuzin L, Yazdanpanah Y. Efficacy of new antiretroviral drugs in treatmentexperienced HIV-infected patients: a systematic review and meta-analysis of recent randomized controlled trials. HIV Med 2012; 13: 148-155.

70 Cahn P, Pozniak AL, Mingrone H et al. Dolutegravir versus raltegravir in antiretroviral-experienced, integrase-inhibitornaive adults with HIV: week 48 results from the randomised, double-blind, non-inferiority SAILING study. Lancet 2013; 382: 700-708.

71 Llibre J, Toro J, Clotet B et al. Withdrawing inactive NRTIs in subjects with suppressed viremia: a randomized trial. Conference on Retroviruses and Opportunistic Infections. February 2015. Seattle, WA, USA [Abstract 553].

72 Tashima K, Smeaton L, Andrade A et al. Omitting NRTI from ARV regimens is not inferior to adding NRTI in treatment-experienced HIV+ subjects failing a protease inhibitor regimen: The ACTG OPTIONS Study. 20th Conference on Retroviruses and Opportunistic Infections. March 2013. Atlanta, GA, USA [Abstract 153LB].

73 Larder BA, Kemp SD, Harrigan PR. Potential mechanism for sustained antiretroviral efficacy of AZT-3TC combination therapy. Science 1995; 269: 696-699.

74 Quan Y, Brenner BG, Oliveira M, Wainberg MA. Lamivudine can exert a modest antiviral effect against human immunodeficiency virus type 1 containing the M184V mutation. Antimicrob Agents Chemother 2003; 47: 747-754.

75 Eron JJ, Benoit SL, Jemsek J et al. Treatment with lamivudine, zidovudine, or both in HIV-positive patients with 200 to 500 CD4 + cells per cubic millimeter. North American HIV Working Party. N Engl J Med 1995; 333: 1662-1669.

76 Gianotti N, Tiberi S, Menzo S et al. HIV-1 replication capacity and genotype changes in patients undergoing treatment interruption or lamivudine monotherapy. J Med Virol 2008; 80: 201-208.

77 Castagna A, Danise A, Menzo S et al. Lamivudine monotherapy in HIV-1-infected patients harbouring a lamivudine-resistant virus: a randomized pilot study (E184V study). AIDS 2006; 20: 795-803.

78 Wainberg MA, Miller MD, Quan Y et al. In vitro selection and characterization of HIV-1 with reduced susceptibility to PMPA. Antivir Ther 1999; 4: 87-94.

79 Miller MD, Margot N, Lu B et al. Genotypic and phenotypic predictors of the magnitude of response to tenofovir disoproxil fumarate treatment in antiretroviral-experienced patients. J Infect Dis 2004; 189: 837-846.

80 Vingerhoets J, Peeters M, Azijn H et al. An update of the list of NNRTI mutations associated with decreased virologic response to etravirine (ETR): multivariate analyses on the 
pooled DUET -1 and DUET 2 clinical trial data. XVII International Drug Resistance Workshop. June 2008. Sitges, Spain [Abstract 24].

81 Lambert-Niclot S, Flandre P, Canestri A et al. Factors associated with the selection of mutations conferring resistance to protease inhibitors (PIs) in PI-experienced patients displaying treatment failure on darunavir. Antimicrob Agents Chemother 2008; 52: 491-496.

82 Parkin N, Vhappey C. Protease mutations associated with higher or lower than expected tipranavir (TPV) susceptibility based up on the TPV mutation score. 13th Conference on Retroviruses and Opportunistic Infections. February 2006. Denver, CO, USA [Abstract 637].

83 Nozza S, Galli L, Visco F et al. Raltegravir, maraviroc, etravirine: an effective protease inhibitor and nucleoside reverse transcriptase inhibitor-sparing regimen for salvage therapy in HIV-infected patients with triple-class experience. AIDS 2010; 24: 924-928.

84 Cahn P, Fourie J, Grinsztejn B et al. Week 48 analysis of once-daily vs. twice-daily darunavir/ritonavir in treatmentexperienced HIV-1-infected patients. AIDS 2011; 25: 929939.

85 Landman R, Capitant C, Descamps D et al. Efficacy and safety of ritonavir-boosted dual protease inhibitor therapy in antiretroviral-naive HIV-1-infected patients: the 2IP ANRS 127 study. J Antimicrob Chemother 2009; 64: 118-125.

86 Miller V, Sabin C, Hertogs K et al. Virological and immunological effects of treatment interruptions in HIV-1 infected patients with treatment failure. AIDS 2000; 14 : 2857-2867.

87 Raffanti SP, Fusco JS, Sherrill BH et al. Effect of persistent moderate viremia on disease progression during HIV therapy. J Acquir Immune Defic Syndr 2004; 37: 1147-1154.
88 Deeks SG, Wrin T, Liegler T et al. Virologic and immunologic consequences of discontinuing combination antiretroviral-drug therapy in HIV-infected patients with detectable viremia. $N$ Engl J Med 2001; 344: 472-480.

89 Lawrence J, Mayers DL, Hullsiek KH et al. Structured treatment interruption in patients with multidrug-resistant human immunodeficiency virus. N Engl J Med 2003; 349: 837-846.

90 Ledergerber B, Lundgren JD, Walker AS et al. Predictors of trend in CD4-positive T-cell count and mortality among HIV-1-infected individuals with virological failure to all three antiretroviral-drug classes. Lancet 2004; 364: 51-62.

91 Kristiansen TB, Pedersen AG, Eugen-Olsen J et al. Genetic evolution of HIV in patients remaining on a stable HAART regimen despite insufficient viral suppression. Scand $J$ Infect Dis 2005; 37: 890-901.

92 Hatano H, Hunt P, Weidler J et al. Rate of viral evolution and risk of losing future drug options in heavily pretreated, HIV-infected patients who continue to receive a stable, partially suppressive treatment regimen. Clin Infect Dis 2006; 43: 1329-1336.

93 Murray JS, Elashoff MR, Iacono-Connors LC et al. The use of plasma HIV RNA as a study endpoint in efficacy trials of antiretroviral drugs. AIDS 1999; 13: 797-804.

94 Lataillade M, Zhou N, Joshi SR et al. HIV-1 attachment inhibitor prodrug BMS-663068 in antiretroviral-experienced subjects: analysis of emergent viral drug resistance through 48 weeks of follow-up. 8th IAS Conference on HIV Pathogenesis, Treatment and Prevention. July 2015. Vancouver, Canada [Abstract TUPEB284]. 


\subsection{Special populations}

\subsection{HIV and TB co-infection}

This guidance provides a brief summary of the key statements and recommendations regarding prescribing ART in HIV-positive individuals co-infected with TB. It is based on the BHIVA guidelines for the treatment of $\mathrm{TB} /$ HIV coinfection 2011 [1], which should be consulted for further information. The full version of the guidelines is available on the BHIVA website (www.bhiva.org/TB-HIVcoinfection-guidelines.aspx).

\subsubsection{When to start ART in TB/HIV co infection}

\subsubsection{Recommendations}

- We recommend all patients with HIV TB co-infection start ART (1B).

- We recommend individuals with $\mathrm{CD} 4$ cell count $<50$ cells $/ \mu \mathrm{L}$ start ART as soon as TB treatment is tolerated and wherever possible within 2 weeks (1B).

- We recommend that for individuals with CD4 cell counts $\geq 50$ cells $/ \mu \mathrm{L}$, ART can be deferred until between 8 and 12 weeks of TB treatment, especially when there are difficulties with drug-drug interactions, adherence and toxicities (1B). (Although the data suggest a cut-off of 50 cells $/ \mu \mathrm{L}$, because of the daily variability in $\mathrm{CD} 4$ cell count, a cut-off of 100 cells $/ \mu \mathrm{L}$ may be more appropriate.)

8.1.1.2 Auditable outcome

- Proportion of individuals with CD4 cell count $<100$ cells $/ \mu$ L started on ART within 2 weeks of starting TB therapy.

\subsubsection{Rationale}

In TB/HIV co-infection, cohort and randomised trial data show that the short-term risk of developing further AIDSdefining events and death is higher if ART is delayed until after the first 8 weeks of TB therapy has been completed. In those with a CD4 cell count less than 50 cells/ $\mu \mathrm{L}$, compared to those with greater than 200 cells $/ \mu \mathrm{L}$, outcomes were best when ART was started as soon as practicable and within 2 weeks of initiation of TB therapy [2-5]. As there is marked intra-individual variability in CD4 cell count [6], particularly amongst individuals with low counts, a threshold of 100 cells $/ \mu \mathrm{L}$ may be more practicable. In one study of HIV-associated TB meningitis, no difference in mortality was shown when starting ART early or late [7] but we recommend the guidance above be followed in this situation and consider monitoring of cerebrospinal fluid (CSF) pressure.

Commencement of ART in individuals with low CD4 cell counts and TB is associated with a large pill burden and higher rates of toxicity, drug interactions and immune reconstitution disorders. These issues can be reduced in individuals with CD4 cell counts of more than 100 cells $/ \mu \mathrm{L}$ who can safely delay starting ART until 8 weeks of TB treatment have been completed. Individuals with HIV and a CD4 cell count $>350$ cells $/ \mu \mathrm{L}$ have a low risk of HIV disease progression or death during the subsequent 6 months of TB treatment, depending on age and viral load. There are limited data in this subgroup and another option is for ART to be withheld until the short-course of TB treatment is completed but with regular monitoring of CD4 cell count.

\subsubsection{What to start in TB/HIV co infection}

8.1.2.1 Recommendations

- We recommend efavirenz in combination with tenofovir-DF and emtricitabine as first-line ART (1B) in TB/ HIV co-infection.

- We recommend that when rifampicin is used with efavirenz, standard doses of efavirenz are given whatever the body weight [8] (1B).

- We suggest that raltegravir can be used as an alternative to efavirenz but should be used with caution (2C).

- We suggest dolutegravir is a possible alternative agent to raltegravir (for which there is currently little evidence) but the dose should be increased to $50 \mathrm{mg}$ twice daily (2D).

- We recommend frequent viral load monitoring if INIs are used (1C).

- We recommend that rifampicin is not used with either nevirapine or a regimen containing ritonavir or cobicistat (1C).

- We recommend that where effective ART necessitates the use of ritonavir or cobicistat, that rifabutin is used instead of rifampicin (1C).

8.1.2.2 Auditable outcome

- Proportion of patients with active TB on anti-TB therapy started on ART containing efavirenz.

8.1.2.3 Rationale

HIV-related TB should be treated with a regimen including a rifamycin for the full course of TB treatment, unless there is rifamycin resistance or intolerance. Rifamycins 
frequently interact with ARV medications and can be associated with similar toxicities, notably rash and hepatitis. Efavirenz is the preferred third agent for ART during TB treatment because of its efficacy in randomised controlled trials [9].

No weight adjustment of efavirenz dose is required as a randomised controlled trial has shown that the standard adult efavirenz dose (600 mg daily) together with two NRTIs is well tolerated and was efficacious in achieving complete viral suppression among adults on concomitant rifampicin-based TB treatment independent of weight [8].

Integrase inhibitors such as raltegravir and dolutegravir should be prescribed with caution with concomitant rifampicin as it decreases serum levels of raltegravir by 40-61\% and trough levels are still low after doubling the dose of raltegravir [10]. A Phase 2 trial of standard (400 mg twice daily) and double dose (800 mg twice daily) raltegravir has showed acceptable virological outcomes [11]. Pharmacokinetic data suggest that dolutegravir can be used with rifampicin but the dolutegravir needs to be administered at $50 \mathrm{mg}$ twice daily [12].

When co-administered with rifampicin, concentrations of standard-dose PIs, with or without ritonavir or cobicistat, are decreased below therapeutic targets, and so cannot be recommended [13-16]. Changing the dosing of the $\mathrm{PI} / \mathrm{r}$ has resulted in increased rates of hepatotoxicity [1618]. Elvitegravir cannot be administered with rifampicin as it requires boosting.

Rifabutin has little effect on the concentrations of $\mathrm{PI} / \mathrm{r}$ but rifabutin concentrations are altered when the PI is taken together with ritonavir or cobicistat. Current recommendations are to reduce the dose and/or frequency of rifabutin. Atazanavir/r can be prescribed with rifabutin at a dose of $150 \mathrm{mg}$ thrice weekly. As darunavir/r also acts as an inducer, rifabutin $150 \mathrm{mg}$ once daily can be given to reduce the theoretical risk of rifamycin resistance due to a risk of sub-therapeutic rifabutin concentrations; this may, however, be associated with increased side effects [19-21]. Recent data suggest rifabutin at $150 \mathrm{mg} /$ day maintains adequate levels with lopinavir/r [22]. Therapeutic drug monitoring may be useful in guiding doses.

Raltegravir and dolutegravir can be used with full-dose daily rifabutin without any dosage adjustment but there are few clinical data to support this strategy.

There are few clinical data to support the use of newer NNRTIs and CCR5 receptor antagonists with rifampicin or rifabutin. We recommend that physicians who are considering using these drugs review pharmacokinetic and other data summarised in the current BHIVA guidelines for treatment of TB/HIV co-infection [1]. As new anti-tuberculous agents become available, drug-drug interactions and the potential for overlapping toxicities should be considered when selecting ART.

\subsubsection{References}

1 Pozniak AL, Coyne KM, Miller RF et al. British HIV Association guidelines for the treatment of TB/HIV coinfection 2011. HIV Med 2011; 12: 517-524.

2 Abdool Karim SS, Naidoo K, Grobler A et al. Timing of initiation of antiretroviral drugs during tuberculosis therapy. N Engl J Med 2010; 362: 697-706.

3 Blanc FX, Sok T, Laureillard D et al. Earlier versus later start of antiretroviral therapy in HIV-infected adults with tuberculosis. N Engl J Med 2011; 365: 1471-1481.

4 Dean GL, Edwards SG, Ives NJ et al. Treatment of tuberculosis in HIV-infected persons in the era of highly active antiretroviral therapy. Aids 2002; 16: 75-83.

5 Havlir DV, Kendall MA, Ive P et al. Timing of antiretroviral therapy for HIV-1 infection and tuberculosis. $N$ Engl J Med 2011; 365: 1482-1491.

6 Hughes MD, Stein DS, Gundacker HM et al. Within-subject variation in CD4 lymphocyte count in asymptomatic human immunodeficiency virus infection: implications for patient monitoring. J Infect Dis 1994; 169: 28-36.

7 Torok ME, Yen NT, Chau TT et al. Timing of initiation of antiretroviral therapy in human immunodeficiency virus (HIV)-associated tuberculous meningitis. Clin Infect Dis 2011; 52: 1374-1383.

8 Luetkemeyer AF, Rosenkranz SL, Lu D et al. Relationship between weight, efavirenz exposure, and virologic suppression in HIV-infected patients on rifampin-based tuberculosis treatment in the AIDS Clinical Trials Group A5221 STRIDE Study. Clin Infect Dis 2013; 57: 586-593.

9 Boulle A, Van Cutsem G, Cohen K et al. Outcomes of nevirapine- and efavirenz-based antiretroviral therapy when coadministered with rifampicin-based antitubercular therapy. Jama 2008; 300: 530-539.

10 Wenning LA, Hanley WD, Brainard DM et al. Effect of rifampin, a potent inducer of drug-metabolizing enzymes, on the pharmacokinetics of raltegravir. Antimicrob Agents Chemother 2009; 53: 2852-2856.

11 Grinsztejn B, De Castro N, Arnold V et al. Raltegravir for the treatment of patients co-infected with HIV and tuberculosis (ANRS 12180 Reflate TB): a multicentre, phase 2, non-comparative, open-label, randomised trial. Lancet Infect Dis 2014; 14: 459-467.

12 Dooley KE, Sayre P, Borland J et al. Safety, tolerability, and pharmacokinetics of the HIV integrase inhibitor dolutegravir given twice daily with rifampin or once daily with rifabutin: results of a phase 1 study among healthy subjects. J Acquir Immune Defic Syndr 2013; 62: 21-27.

13 Acosta EP, Kendall MA, Gerber JG et al. Effect of concomitantly administered rifampin on the 
pharmacokinetics and safety of atazanavir administered twice daily. Antimicrob Agents Chemother 2007; 51: 3104 3110.

14 la Porte CJ, Colbers EP, Bertz R et al. Pharmacokinetics of adjusted-dose lopinavir-ritonavir combined with rifampin in healthy volunteers. Antimicrob Agents Chemother 2004; 48: 1553-1560.

15 Ribera E, Azuaje C, Lopez RM et al. Pharmacokinetic interaction between rifampicin and the once-daily combination of saquinavir and low-dose ritonavir in HIVinfected patients with tuberculosis. J Antimicrob Chemother 2007; 59: 690-697.

16 Schmitt C, Riek M, Winters K et al. Unexpected hepatotoxicity of rifampin and saquinavir/ritonavir in healthy male volunteers. Arch Drug Inf 2009; 2: 8-16.

17 Haas DW, Koletar SL, Laughlin L et al. Hepatotoxicity and gastrointestinal intolerance when healthy volunteers taking rifampin add twice-daily atazanavir and ritonavir. $J$ Acquir Immune Defic Syndr 2009; 50: 290-293.

18 Nijland HM, L'Homme RF, Rongen GA et al. High incidence of adverse events in healthy volunteers receiving rifampicin and adjusted doses of lopinavir/ritonavir tablets. Aids 2008; 22: 931-935.

19 Jenny-Avital ER, Joseph K. Rifamycin-resistant Mycobacterium tuberculosis in the highly active antiretroviral therapy era: a report of 3 relapses with acquired rifampin resistance following alternate-day rifabutin and boosted protease inhibitor therapy. Clin Infect Dis 2009; 48: 1471-1474.

20 Narita M, Stambaugh JJ, Hollender ES et al. Use of rifabutin with protease inhibitors for human immunodeficiency virus-infected patients with tuberculosis. Clin Infect Dis 2000; 30: 779-783.

21 Tseng AL, Walmsley SL. Rifabutin-associated uveitis. Ann Pharmacother 1995; 29: 1149-1155.

22 Lan NT, Thu NT, Barrail-Tran A et al. Randomised pharmacokinetic trial of rifabutin with lopinavir/ritonavirantiretroviral therapy in patients with HIV-associated tuberculosis in Vietnam. PLoS One 2014; 9: e84866.

\subsection{Hepatitis B and C virus co-infection}

\subsubsection{When to start ART?}

Table 8.2.1 Summary recommendations for the treatment of hepatitis B and C co-infection

\begin{tabular}{|c|c|c|c|}
\hline $\begin{array}{l}\text { HBV requiring } \\
\text { treatment* }\end{array}$ & $\begin{array}{l}\text { HBV not } \\
\text { requiring } \\
\text { treatment }\end{array}$ & $\begin{array}{l}\text { HCV with immediate } \\
\text { plan to start HCV } \\
\text { treatment* }\end{array}$ & $\begin{array}{l}\text { HCV with no } \\
\text { immediate } \\
\text { plan to } \\
\text { start HCV } \\
\text { treatment }\end{array}$ \\
\hline $\begin{array}{l}\text { Start ART } \\
\text { promptly (1A) } \\
\text { (include } \\
\text { tenofovir-DF } \\
\text { and } \\
\text { emtricitabine) }\end{array}$ & $\begin{array}{l}\text { Start ART (1A) } \\
\quad \text { (include } \\
\text { tenofovir-DF } \\
\text { and } \\
\text { emtricitabine) }\end{array}$ & $\begin{array}{l}\text { Start ART before } \\
\text { HCV treatment } \\
\text { commenced (1C); } \\
\text { acceptable to } \\
\text { defer if CD4 cell } \\
\text { count }>500 \text { cells/ } \\
\mu L . \text { Discuss with } \\
\text { HIV and viral } \\
\text { hepatitis specialist }\end{array}$ & Start ART (1A) \\
\hline
\end{tabular}

*See BHIVA guidelines for the management of hepatitis viruses in adults infected with HIV 2013 [1] for indications to treat hepatitis B and C

\subsubsection{Hepatitis $B$}

8.2.2.1 When to start antiretroviral therapy in HBV coinfection

\subsection{Recommendations}

- We recommend individuals with HIV and hepatitis B virus (HBV) co-infection are treated with fully suppressive ART inclusive of anti-HBV active antivirals, regardless of CD4 cell count (1A).

- We recommend individuals with HIV and HBV coinfection who have an HBV-DNA $\geq 2000 \mathrm{IU} / \mathrm{mL}$ and/or evidence of more than minimal fibrosis (Metavir $\geq \mathrm{F} 2$ ) are treated with fully suppressive ART inclusive of anti-HBV active antivirals promptly (1C).

\subsection{Rationale}

Because of the negative effect of immune depletion on HBV disease progression, the availability of single drugs with high-level dual HBV and HIV antiviral activity, and the increased risk of liver-related deaths in individuals with CD4 cell counts $\geq 500$ cells $/ \mu \mathrm{L}$, co-infected individuals with active HBV disease (HBV viral load $\geq 2000 \mathrm{IU} / \mathrm{mL}$ or Metavir F2 or above) and those with CD4 cell counts below 500 cells $/ \mu \mathrm{L}$ should start ART inclusive of anti$\mathrm{HBV}$ active antivirals without delay. Individuals with CD4 cell counts $\geq 500$ cells $/ \mu \mathrm{L}$ and HBV DNA of $<2000 \mathrm{IU} / \mathrm{mL}$, minimal or no evidence of liver inflammation or fibrosis, and a repeatedly normal ALT should start ART, regardless of CD4 cell count, when they are ready to do so. Anyone 
who chooses to defer treatment should be monitored not less than 6-monthly with HBV DNA and ALT and at least annually for evidence of fibrosis.

For more information on starting treatment for $\mathrm{HBV}$ please refer to the BHIVA guidelines for the management of hepatitis viruses in adults infected with HIV [1].

8.2.2.2 What to start in HBV co-infection

8.2.2.2.1 What to start recommendations

- We recommend tenofovir-DF/emtricitabine as part of a fully suppressive ART combination should be given to all individuals starting HIV treatment (1C).

- We recommend neither lamivudine nor emtricitabine be used as the sole active drug against HBV in ART due to the rapid emergence of HBV resistant to these agents (1B).

- We recommend lamivudine/emtricitabine may be omitted from the ART regimen and tenofovir-DF be given as the sole anti-HBV active agent if there is clinical or genotypic evidence of lamivudine/emtricitabine-resistant HBV or HIV (1D).

\subsection{Rationale}

Tenofovir-DF, emtricitabine and lamivudine are agents that have good antiviral activity against both HIV and HBV. The efficacy of these drugs against HBV has been assessed in randomised trials extending out to 5 years in mono-infected patients [1]. They are recommended agents in these guidelines for the treatment of HIV-1 infection.

All HBV co-infected individuals should commence a regimen containing tenofovir-DF and emtricitabine. Hepatitis B treatment options for patients declining ART are discussed elsewhere [1].

If an individual becomes intolerant or is unable to commence a tenofovir-DF-containing regimen, entecavir should be used if it is active. Because entecavir demonstrates modest anti-HIV activity and can select for HIV resistance, it should only be used in addition to a fully suppressive combination ART regimen. No individual coinfected with HBV should receive a regimen containing lamivudine or emtricitabine monotherapy as its use may result in the selection of the YMDD mutation. TenofovirDF resistance has not been clearly described and resistance is unlikely to provide an explanation for most cases of suboptimal responses to tenofovir-DF. In combination with lamivudine or emtricitabine, it has been demonstrated to be effective at suppressing HBV DNA, inducing $\mathrm{HBeAg}$ seroconversion, and reducing the risk of $\mathrm{HBV}$ breakthrough [1].

Where there is primary non-response or partial response to $\mathrm{HBV}$-active antivirals, or where there is virological breakthrough, assessment of drug adherence and HBV resistance testing should be undertaken. Coinfected individuals who need to start a new ART regimen for reasons such as ART virological failure should ensure that effective anti-HBV therapy is continued in addition to their new ART regimen. Abrupt withdrawal of effective treatment may lead to a flare in HBV replication with liver damage. This may be particularly severe in patients with cirrhosis.

\subsubsection{Hepatitis $C$}

8.2.3.1 When to start antiretroviral therapy in HCV coinfection

\subsection{Recommendations}

- We recommend all individuals with HIV and hepatitis $\mathrm{C}$ virus (HCV) co-infection be assessed for HCV treatment (GPP).

- We recommend commencing ART regardless of CD4 cell count (1A).

- We recommend HCV be considered an additional factor supporting ART in individuals with CD4 $>500$ cells $/ \mu \mathrm{L}$ who are uncertain about commencing ART (2C).

- We suggest treating HCV before commencing ART is an option if there are concerns about drug-drug interactions or adherence (GPP).

\subsection{Rationale}

HIV has an impact on HCV infection. Individuals with HCV co-infection have higher HCV viral loads, faster rates of fibrosis progression and an increased risk of cirrhosis compared to those with HCV alone. End-stage liver disease, hepatocellular carcinoma (HCC) and liver-related death occur more frequently, at an earlier age, and within a shorter time period with the risk of liver-related mortality and HCC increasing as the CD4 cell count declines. The efficacy of pegylated interferon (PEG-IFN) lessens as the CD4 cell count declines (PEG-IFN-free regimens seem to not be affected by HIV markers) and although ART slows the progression of liver disease it is still likely to be faster than in HCV mono-infection.

For these reasons, individuals with HIV and HCV infection with CD 4 cell counts $<500$ cells $/ \mu$ L should start ART; this should be immediate irrespective of whether HCV treatment is planned or not. For those with CD4 cell counts between 350 and 500 cells $/ \mu \mathrm{L}$, initiation of antiHCV treatment should be delayed until after start of ART unless there is an urgent indication for anti-HCV treatment when ART should be commenced as soon as the patient has been stabilised on HCV therapy.

Individuals with a CD4 cell count greater than 500 cells $/ \mu \mathrm{L}$ who defer HCV therapy should be given the 
option to commence ART. If they opt to defer, they should be monitored closely for HIV or hepatitis C disease progression, including at least an annual assessment of liver fibrosis.

8.3.2.2 What to start in HCV co-infection

8.3.2.2.1 Recommendations

- We recommend if individuals are commencing ART, and direct-acting antivirals are not being considered, standard first-line ART should be commenced (GPP).

- We recommend that when direct-acting antivirals are to be used, there is careful consideration of possible drug-drug interactions (1C) and current or archived HIV resistance. All drug interactions should be checked with an expert source (e.g. www.hiv-druginteractions.org).

- We suggest that if abacavir is to be used with ribavirin, the ribavirin should be weight-based dose-adjusted (2C).

\subsubsection{Reference}

1 Wilkins E, Nelson M, Agarwal K et al. British HIV Association guidelines for the management of hepatitis viruses in adults infected with HIV 2013. HIV Med 2013; 14 (Suppl 4): 1-71.

\subsection{HIV-related cancers}

Please see the BHIVA guidelines for HIV-associated malignancies 2014 [1] for further details (www.bhiva.org/malignancy-guidelines.aspx).

\subsubsection{When to start ART?}

\subsubsection{AIDS-defining malignancies}

Kaposi sarcoma, high-grade B-cell non-Hodgkin lymphoma and invasive cervical cancer are all AIDS-defining illnesses and are thus indications to commence ART regardless of CD4 cell count or HIV viral load.

- We recommend that all patients with AIDS-defining malignancies should start ART promptly (1B).

8.3.1.1.1 Kaposi sarcoma (KS)

- We recommend that ART should be started promptly in all individuals diagnosed with KS (1B).

8.3.1.1.2 Non-Hodgkin lymphoma (NHL)

- We recommend that chemotherapy regimens should be combined with ART therapy in Burkitt lymphoma and diffuse large B-cell lymphoma (1B).

- We recommend that all individuals with primary effusion lymphoma (PEL), plasmablastic lymphoma and primary central nervous system lymphoma should be started on ART if not already on it (1C).

\subsection{Cervical cancer}

Women with HIV and cervical intra-epithelial neoplasia (CIN) 2/3 treated by excisional procedures have a significantly higher treatment failure rate than HIV-negative women. A number of studies show such relapse is less frequent in the presence of ART, higher CD4 cell counts or undetectable viral load.

- We suggest that women with CIN2/3 should commence ART promptly (2B).

- We recommend that all women living with HIV who are to be treated with chemo-radiotherapy (CRT) for cervical cancer should start ART promptly (1C).

8.3.1.2 Non-AIDS-defining malignancies

8.3.1.2.1 Anal cancer

- We recommend that all PLWH who are to be treated with chemo-radiotherapy should start ART (1C).

8.3.1.2.2 Hodgkin lymphoma

- We recommend all PLWH and Hodgkin lymphoma should receive ART during chemotherapy (1A).

8.3.1.2.3 Other non-AIDS-defining cancers

- We suggest all PLWH who require chemotherapy or radical radiotherapy should receive concomitant ART unless contraindicated (level of evidence 2C).

\subsubsection{What to start}

- We recommend that all potential interactions between ART, opportunistic infection prophylaxis and cancer therapy should be considered (1C).

- We suggest that all individuals with non-AIDS-defining malignancies who are due to start chemotherapy or radiotherapy should be started on ART unless contraindicated $(2 \mathrm{C})$.

8.3.3 Opportunistic infection prophylaxis in HIVassociated malignancy

- We recommend that all individuals with AIDS-defining malignancies should start ART immediately (1B).

- We suggest that all individuals with non-AIDS-defining malignancies who are due to start chemotherapy or radiotherapy should be started on ART immediately unless contraindicated (2C).

- We recommend that individuals with antibodies against hepatitis $\mathrm{B}$ core antigen ( $\mathrm{HBcAb}$ ) should be treated with prophylactic antivirals in line with BHIVA hepatitis guidelines (1B).

8.3.4 Other considerations from the BHIVA guidelines for HIV-associated malignancies [1]

- We recommend that potential pharmacokinetic interactions between antiretrovirals and systemic anticancer 
therapy are checked prior to administration (with tools such as: www.hiv-druginteractions.org) (1C).

Significant pharmacokinetic and pharmacodynamic interactions have been reported between antiretroviral drugs and systemic anticancer therapies. The mechanisms of the pharmacokinetic interactions include the inhibition and induction by antiretroviral agents of enzymes, especially the cytochrome P450 family and UGT (uridine diphospho-glucuronosyl transferase) isoenzymes, involved in the catabolism and activation of cytotoxic chemotherapy agents. In addition, competition for renal clearance, intracellular phosphorylation and abacavir (ATP binding cassette) transporters, has been hypothesised to contribute to these drug interactions [2]. Similarly, pharmacodynamic interactions, in particular overlapping toxicities between antiretrovirals and systemic anticancer therapy suggest that some drug combinations should be avoided in patients with HIV associated cancers. Much of the guidance on the use of individual antiretroviral agents with systemic anticancer therapy comes from reviews of potential drug interactions rather than from clinical studies [2-4]. The pharmacokinetic interactions between antiretrovirals and systemic anticancer therapy are not confined to cytotoxic chemotherapy agents and extensive interactions with newer targeted therapies such as imatinib, erlotinib, sorafanib, bortezomib and temsirolimus have been described [4].

- We suggest avoiding ritonavir (or cobicistat)-boosted ART in HIV-positive individuals who are to receive cytotoxic chemotherapy agents that are metabolised by the CYP450 enzyme system (2C).

In general, clinically important pharmacokinetic drug interactions with systemic anticancer therapies are most common with ritonavir-boosted protease inhibitor-based ART and most clinicians avoid these combinations where possible. For example, in a cohort study, the rates of severe infections and severe neutropenia following chemotherapy for AIDS-related NHL were significantly higher amongst individuals receiving concomitant PI (mainly ritonavir boosted) than in those on NNRTI-based ART regimens, although there was no difference in survival between the groups [5]. Furthermore, case reports of clinically significant life-threatening interactions between ritonavir-boosted-based ART and docetaxel [6], irinotecan [7], vinblastine [8] have been published.

- We suggest avoiding atazanavir in HIV-positive individuals who are to receive irinotecan (2C).

The camptothecin cytotoxic agent, irinotecan, is extensively metabolised by UGT1A1 isoenzymes that are inhibited by atazanavir [9]. In people with Gilbert's syndrome, who have a congenital deficiency of UGT1A1, irinotecan administration has led to life-threatening toxicity [10].

- We suggest switching antiretroviral agents in HIVpositive patients who are to receive cytotoxic chemotherapy agents to avoid severe and/or overlapping toxicities (2C).

- Medicines reconciliation prior to chemotherapy to minimise potential pharmacokinetic interactions and overlapping toxicity should be undertaken by an experienced pharmacist (GPP).

Both antiretroviral agents and systemic anticancer therapies have substantial toxicity and where these overlap it is likely that the risk of toxicity is greater. For example zidovudine commonly causes myelosuppression and anaemia [11], which are also frequent side effects of cytotoxic chemotherapy and so these should not be coprescribed where possible. Similarly, stavudine, didanosine and zalcitabine cause peripheral neuropathy [12], a common toxicity of taxanes and vinca alkaloids, so coprescribing should be avoided.

\subsubsection{References}

1 Bower M, Palfreeman A, Alfa-Wali M et al. British HIV Association guidelines for HIV-associated malignancies 2014. HIV Med 2014; 15 (Suppl 2): 1-92.

2 Rudek MA, Flexner C, Ambinder RF. Use of antineoplastic agents in patients with cancer who have HIV/AIDS. Lancet Oncol 2011; 12: 905-912.

3 Antoniou T, Tseng AL. Interactions between antiretrovirals and antineoplastic drug therapy. Clin Pharmacokinet 2005; 44: 111-145.

4 Deeken JF, Pantanowitz L, Dezube BJ. Targeted therapies to treat non-AIDS-defining cancers in patients with HIV on HAART therapy: treatment considerations and research outlook. Curr Opin Oncol 2009; 21: 445-454.

5 Bower M, McCall-Peat N, Ryan N et al. Protease inhibitors potentiate chemotherapy-induced neutropenia. Blood 2004; 104: 2943-2946.

6 Mir 0, Dessard-Diana B, Louet AL et al. Severe toxicity related to a pharmacokinetic interaction between docetaxel and ritonavir in HIV-infected patients. Br J Clin Pharmacol 2010; 69: 99-101.

7 Corona G, Vaccher E, Sandron S et al. Lopinavir-ritonavir dramatically affects the pharmacokinetics of irinotecan in HIV patients with Kaposi's sarcoma. Clin Pharmacol Ther 2008; 83: 601-606.

8 Kotb R, Vincent I, Dulioust A et al. Life-threatening interaction between antiretroviral therapy and vinblastine in 
HIV-associated multicentric Castleman's disease. Eur J

Haematol 2006; 76: 269-271.

9 Fujita K, Sparreboom A. Pharmacogenetics of irinotecan disposition and toxicity: a review. Curr Clin Pharmacol 2010; 5: 209-217.

10 Wasserman E, Myara A, Lokiec F et al. Severe CPT-11 toxicity in patients with Gilbert's syndrome: two case reports. Ann Oncol 1997; 8: 1049-1051.

11 Moyle G, Sawyer W, Law M et al. Changes in hematologic parameters and efficacy of thymidine analogue-based, highly active antiretroviral therapy: a meta-analysis of six prospective, randomized, comparative studies. Clin Ther 2004; 26: 92-97.

12 Moyle GJ, Sadler M. Peripheral neuropathy with nucleoside antiretrovirals: risk factors, incidence and management.

Drug Saf 1998; 19: 481-494.

\subsection{HIV-associated neurocognitive impairment}

\subsubsection{Introduction}

With the widespread use of effective combination antiretroviral therapy, the incidence of severe HIV-associated cerebral disease has declined dramatically [1]; however, more subtle forms of brain disease, known as HIV-associated neurocognitive (NC) disorders are reported to remain prevalent [2]. This NC deficit may present with a wide spectrum of clinical symptoms and typically includes patterns involving ineffective learning and difficulties in decision making or executive function, rather than pure difficulties in formulating new memory (the cortical defect typical of Alzheimer's disease [3]).

Given the changing picture of this disease, a research nomenclature system has proposed classifying subjects with abnormal neuropsychological testing results in to three categories based on symptoms, measured via the activities of daily living (ADL) scale [2]. Subjects with abnormal neuropsychiatric testing results, who are otherwise asymptomatic are classified as having HIVassociated asymptomatic neurocognitive impairment (ANI); those who are mildly symptomatic are classified as having HIV-associated mild neurocognitive disorder (MND); and those who are severely symptomatic are classified as having HIV-associated dementia (HAD). The clinical relevance of ANI, namely asymptomatic subjects with abnormal results on neuropsychological testing, remains unclear. Although the presence of ANI has been linked to progression of symptomatic cognitive impairment in one longitudinal study [4], the presence of other co-morbidities was also higher in individuals progressing to symptomatic cognitive impairment. These guidelines will focus only on symptomatic subjects.

Reports describing rates of NC impairment vary with some groups describing up to $50 \%$ of HIV-positive subjects meeting the above criteria [5]. However, such reports should be interpreted with caution as asymptomatic subjects are often included and not all reports correct for effective antiretroviral use. More selective cohorts including only aviraemic and symptomatic subjects suggest the prevalence of MND to be between 6 and 19\% [6,7].

Risk factors for the development of NC disorders are poorly understood and are likely to be multifactorial including both HIV disease factors [8] and concomitant diseases [9]. Although it is possible that the choice of combination antiretroviral therapy subjects receive may influence NC function, this is a controversial area without definitive evidence. The following recommendations apply to individuals with symptomatic HIV-associated NC disorders.

\subsubsection{When to start ART}

\subsubsection{Recommendation}

- We recommend individuals with symptomatic HIV-associated neurocognitive disorders start ART immediately, irrespective of CD4 cell count (1C).

8.4.2.2 Rationale

Current evidence suggests NC function improves after commencing antiretroviral therapy for the first time [10] in both cognitively symptomatic [11] and asymptomatic [12] subjects. However, these studies have been undertaken in individuals with other indications to commence antiretroviral therapy, in general with CD4 cell counts $<350$ cells $/ \mu$ L. For subjects with higher CD4 cell counts, the ongoing neurology substudy in the START study will prospectively assess NC function in HIV-positive subjects commencing antiretroviral therapy at an earlier stage of HIV disease.

In the absence of scientific data, in cognitively symptomatic subjects with higher CD4 cell counts, a recommendation to commence antiretroviral therapy [13] is first based on the observed improvements in cognitive function reported in subjects with lower CD4 cell counts commencing therapy [10]; and secondly, in order to avoid a future decline in CD4 cell count in such subjects, given the well-described association between low nadir CD4 cell count and NC impairment [8].

Sub-optimal adherence to therapy may occur more frequently in subjects with NC impairment, hence adequate support services to optimise adherence are essential. 


\subsubsection{What to start with}

\subsubsection{Recommendations}

- We recommend individuals with HIV-associated NC disorders start standard combination ART regimens (1C).

- We recommend avoiding efavirenz-containing regimens in individuals with HIV-associated NC disorders (1C).

8.4.3.2 Rationale

8.4.3.2.1 Including zidovudine in a regimen

Although during the earlier years of antiretroviral therapy, clear benefits on cerebral function of individual antiretroviral drugs such as zidovudine were reported [14] and the benefits of combination therapy overall described [10], data are sparse regarding any differences in these benefits between individual agents or combinations. Within cohort studies, the use of the NRTI class within antiretroviral regimens has been associated with a reduced risk of severe $\mathrm{HAD}$ [15] compared to the use of other regimens; however, the confounders of a cohort study limit the interpretation of these data.

The improvements in NC function observed with zidovudine monotherapy [14] and the greater improvements in NC function observed with a zidovudine-containing quadruple nucleoside regimen compared to other ART regimens [16], raise the possibility of selecting a zidovudine-containing antiretroviral regimen in subjects with NC impairment. Conversely, a lack of comparator data for zidovudine monotherapy, and potential toxicities arising from zidovudine use, may limit the relevance of these data. Of note, further to peripheral toxicities, which are well described with zidovudine use, biomarker data suggest there may also be central nervous system toxicities associated with the use of zidovudine-containing regimens [17].

8.4.3.2.2 Considerations regarding the CPE score

Recently, attempts have been made to establish a relationship between cognitive function and CNS antiretroviral drug delivery based on an antiretroviral scoring system known as the clinical penetration effectiveness (CPE) score [18]. The CPE score aims to rationally score the cerebral effects of individual antiretroviral agents. However the system is predominantly designed around pharmacokinetic modelling rather than pharmacodynamic endpoints such as data describing changes in NC function. Studies that have assessed the correlation between the CPE scores of antiretroviral regimens with NC function report conflicting findings with some cohorts reporting a positive association $[19,20]$ while other cohorts describe a negative association [21,22]. In a small prospective study no differences in cognitive outcomes were observed in subjects randomised to higher CPE score-containing antiretroviral regimens compared to standard therapies [7]. Given these factors, the CPE score should not influence therapeutic decisions in subjects with NC impairment commencing antiretroviral therapy.

8.4.3.2.3 Considerations for not including efavirenz

Although early neuropsychiatric side effects are widely recognised and common with efavirenz therapy, recent reports have highlighted concerns regarding poorer NC function being associated with efavirenz-containing regimens. One cohort study has reported poorer cognitive function to be associated with current efavirenz use [23]. Two randomised controlled studies have assessed the cognitive effects of efavirenz. In one small study, improvements in cognitive function were poorer in those allocated efavirenz-containing therapy [16] and in a recent large study, the time to development of cognitive impairment was quicker in subjects allocated efavirenzcontaining therapy [24]. Antiretroviral switch studies have reported improvement in central nervous system symptomatology when modifying therapy to non-efavirenz-containing regimens $[25,26]$. Conversely, virological efficacy and low pill burden are key components of therapies for individuals with NC impairment. We therefore only recommend avoiding efavirenz-containing regimens in situations where other regimens with comparable virological efficacy and convenience can be initiated.

\subsection{Novel antiretroviral strategies}

Novel antiretroviral strategies, including protease-inhibitor monotherapy continue to be of interest given the potential for reduced long-term toxicities. Concerns have been raised regarding the cerebral effects of PI monotherapy [27], and dual therapies [28]. Such concerns are based on the hypotheses that novel strategies comprise only one or two effective antiretroviral agents that may not adequately suppress ongoing HIV replication in sanctuary sites such as the CNS and are based on pharmacokinetic modelling that suggests not all PIs have optimal penetration across the blood-brain barrier [18]. Furthermore, isolated cases describing the evolution of CNS disease in previously stable HIV-infected subjects receiving PI monotherapy have been reported [29].

One study was specifically designed to assess the cerebral effects of lopinavir/ritonavir monotherapy [30]. However, this study was terminated early due to a lack of efficacy in the plasma compartment. Although cases of CNS disease were reported within this study, such results must be interpreted with caution as virological endpoints in the plasma compartment were not met and therefore such cases may be driven by poor antiretroviral efficacy per se, rather than distinct CNS disease itself [31]. 
In the PIVOT study, the largest study of PI monotherapy to date, no differences in cognitive-function parameters were noted over 5 years of follow up in subjects randomised to continue standard therapy versus commence PI monotherapy [32]. Although reassuring, this study did not recruit neurologically symptomatic subjects and only enrolled subjects on antiretroviral therapy with undetectable viraemia.

Given the above theoretical concerns regarding the CNS activity of novel antiretroviral strategies, and for the majority of HIV-infected subjects it may be possible to select other antiretroviral regimens, we suggest this approach is currently avoided where possible in neurologically symptomatic subjects.

\subsubsection{Continuing or worsening NC impairment despite ART}

8.4.4.1 Recommendations

Best practice management should include (GPP):

- Reassessment for confounding conditions.

- Assessment of CSF HIV RNA and genotyping of CSF HIV RNA.

- In subjects with detectable CSF HIV RNA, modifications to antiretroviral therapy should be based on paired plasma and CSF genotypic results.

8.4.4.2 Rationale

Several randomised controlled studies, assessing both intensification of antiretroviral therapy with new antiretroviral agents [7,33] and with adjunctive therapies [34-37] have been published. Unfortunately, none of these studies describes improvements in cognition subsequent to the study interventions. Without evidence-based interventions, a best-practice approach based on the current literature is outlined.

As HIV-associated NC disorders are diagnoses of exclusion, re-evaluation of subjects with ongoing NC impairment despite antiretroviral therapy for confounding conditions, with expert input from other clinical specialties such as psychiatry, neurology and neuropsychology is recommended and, where possible, input from an HIV-neurology service. Given the presence of non-infectious comorbidities reported to be a risk factor for NC impairment [9], such conditions should be optimally managed.

Assessment of CSF HIV RNA and genotypic analysis of CSF RNA may be useful tools in the management of subjects with ongoing NC for the following reasons: first, data from cohorts of untreated HIV-positive subjects would suggest CSF HIV RNA to be greater in subjects with HIV-associated dementia and cognitive decline [3840] and therefore suppression of CSF HIV RNA may be beneficial for cognitive function. Secondly, in subjects with ongoing NC impairment, higher degrees of genetic diversity between HIV viral strains in the CSF and plasma compartment may exist [41], even in subjects with undetectable plasma HIV RNA [4,42]. Therefore, assessment for CSF HIV resistance is justified in order to tailor antiretroviral therapy.

Management should also involve consideration of any potential antiretroviral toxicities and side effects. For instance, a trial of switching from an efavirenz-containing regimen to an alternative may be considered along with any potential disadvantages of treatment modifications as outlined above.

\subsubsection{References}

1 Dore GJ, Correll PK, Li Y et al. Changes to AIDS dementia complex in the era of highly active antiretroviral therapy. AIDS 1999; 13: 1249-1253.

2 Antinori A, Arendt G, Becker JT et al. Updated research nosology for HIV-associated neurocognitive disorders. Neurology 2007; 69: 1789-1799.

3 Valcour V, Paul R, Chiao S et al. Screening for cognitive impairment in human immunodeficiency virus. Clin Infect Dis 2011; 53: 836-842.

4 Grant I, Franklin DR Jr, Deutsch R et al. Asymptomatic HIV-associated neurocognitive impairment increases risk for symptomatic decline. Neurology 2014; 82: 2055-2062.

5 Heaton RK, Clifford DB, Franklin DR Jr et al. HIV-associated neurocognitive disorders persist in the era of potent antiretroviral therapy: CHARTER Study. Neurology 2011; 75: 2087-2096.

6 Simioni S, Cavassini M, Annoni JM et al. Cognitive dysfunction in HIV patients despite long-standing suppression of viremia. AIDS 2010; 24: 1243-1250.

7 Ellis RJ, Letendre S, Vaida F et al. Randomized trial of central nervous system-targeted antiretrovirals for HIVassociated neurocognitive disorder. Clin Infect Dis 2014; 58: 1015-1022.

8 Ellis RJ, Badiee J, Vaida F et al. CD4 nadir is a predictor of HIV neurocognitive impairment in the era of combination antiretroviral therapy. AIDS 2011; 25: 1747-1751.

9 Wright EJ, Grund B, Robertson K et al. Cardiovascular risk factors associated with lower baseline cognitive performance in HIV-positive persons. Neurology 2011; 75: 864-873.

10 Al-Khindi T, Zakzanis KK, van Gorp WG. Does antiretroviral therapy improve HIV-associated cognitive impairment? A quantitative review of the literature. J Int Neuropsychol Soc 2011; 1-14.

11 Cysique LA, Vaida F, Letendre S et al. Dynamics of cognitive change in impaired HIV-positive patients initiating antiretroviral therapy. Neurology 2009; 73: 342348.

12 Winston A, Puls R, Kerr SJ et al. Dynamics of cognitive change in HIV-infected individuals commencing three 
different initial antiretroviral regimens: a randomized, controlled study. HIV Med 2012; 13: 245-251.

13 Winston A, Vera JH. Can antiretroviral therapy prevent HIV-associated cognitive disorders? Curr Opin HIV AIDS 2014; 9: 11-16.

14 Schmitt FA, Bigley JW, McKinnis R et al. Neuropsychological outcome of zidovudine (AZT) treatment of patients with AIDS and AIDS-related complex. $N$ Engl $J$ Med 1988; 319: 1573-1578.

15 d'Arminio Monforte A, Cinque P, Mocroft A et al. Changing incidence of central nervous system diseases in the EuroSIDA cohort. Ann Neurol 2004; 55: 320-328.

16 Winston A, Duncombe C, Li PC et al. Does choice of combination antiretroviral therapy (cART) alter changes in cerebral function testing after 48 weeks in treatment-naive, HIV-1-infected individuals commencing cART? A randomized, controlled study. Clin Infect Dis 2010; 50: 920-929.

17 Schweinsburg BC, Taylor MJ, Alhassoon OM et al. Brain mitochondrial injury in human immunodeficiency virusseropositive $(\mathrm{HIV}+)$ individuals taking nucleoside reverse transcriptase inhibitors. J Neurovirol 2005; 11: 356-364.

18 Letendre S, Marquie-Beck J, Capparelli E et al. Validation of the CNS Penetration-Effectiveness rank for quantifying antiretroviral penetration into the central nervous system. Arch Neurol 2008; 65: 65-70.

19 Tozzi V, Balestra P, Salvatori MF et al. Changes in cognition during antiretroviral therapy: comparison of 2 different ranking systems to measure antiretroviral drug efficacy on HIV-associated neurocognitive disorders. J Acquir Immune Defic Syndr 2009; 52: 56-63.

20 Smurzynski M, Wu K, Letendre S et al. Effects of central nervous system antiretroviral penetration on cognitive functioning in the ALLRT cohort. AIDS 2011; 25: 357-365.

21 Marra CM, Zhao Y, Clifford DB et al. Impact of combination antiretroviral therapy on cerebrospinal fluid HIV RNA and neurocognitive performance. AIDS 2009; 23: 1359-1366.

22 Ashraf T, Robillard K, Chan GN, Bendayan R. Role of CNS transporters in the pharmacotherapy of HIV-1 associated neurological disorders. Curr Pharm Des 2014; 20: 1543-1563.

23 Ciccarelli N, Fabbiani M, Di Giambenedetto S et al. Efavirenz associated with cognitive disorders in otherwise asymptomatic HIV-infected patients. Neurology 2011; 76: 1403-1409.

24 Zhang F, Mu W, Heaton R et al. Randomized clinical trial of antiretroviral therapy for prevention of HAND. Conference on Retroviruses and Opportunistic Infections. February 2015. Seattle, WA, USA [Abstract 56].

25 Waters L, Fisher M, Winston A et al. A phase IV, doubleblind, multicentre, randomized, placebo-controlled, pilot study to assess the feasibility of switching individuals receiving efavirenz with continuing central nervous system adverse events to etravirine. AIDS 2011; 25: 65-71.

26 Mills AM, Cohen C, Dejesus E et al. Efficacy and safety 48 weeks after switching from efavirenz to rilpivirine using emtricitabine/tenofovir disoproxil fumarate-based singletablet regimens. HIV Clin Trials 2013; 14: 216-223.

27 Perez-Valero I, Bayon C, Cambron I et al. Protease inhibitor monotherapy and the CNS: peace of mind? J Antimicrob Chemother 2011; 66: 1954-1962.

28 Mangioni D, Muscatello A, Sabbatini F et al. A case of CSF viral escape on dual antiretroviral regimen: worth the risk? Clin Infect Dis 2014.

29 Katlama C, Valantin MA, Algarte-Genin M et al. Efficacy of darunavir/ritonavir maintenance monotherapy in patients with HIV-1 viral suppression: a randomized open-label, noninferiority trial, MONOI-ANRS 136. AIDS 2010; 24: 2365-2374.

30 Gutmann C, Cusini A, Gunthard HF et al. Randomized controlled study demonstrating failure of LPV/r monotherapy in HIV: the role of compartment and CD4nadir. AIDS 2010; 24: 2347-2354.

31 Paton NI, Meynard JL, Pulido F et al. Inappropriate claim of 'failure of ritonavir-boosted lopinavir monotherapy in HIV' in the Monotherapy Switzerland/Thailand (MOST) trial. AIDS 2011; 25: 393-394.

32 Paton N, on behalf of the PIVOT Trial Team. The Protease Inhibitor monotherapy Versus Ongoing Triple-therapy Trial (PIVOT). Third Joint Conference of BHIVA and BASHH. April 2014. Liverpool, UK [Abstract 01].

33 Brew BJ, Halman M, Catalan J et al. Factors in AIDS dementia complex trial design: results and lessons from the abacavir trial. PLoS Clin Trials 2007; 2: e13.

34 Schifitto G, Navia BA, Yiannoutsos CT et al. Memantine and HIV-associated cognitive impairment: a neuropsychological and proton magnetic resonance spectroscopy study. AIDS 2007; 21: 1877-1886.

35 Schifitto G, Yiannoutsos CT, Ernst T et al. Selegiline and oxidative stress in HIV-associated cognitive impairment. Neurology 2009; 73: 1975-1981.

36 Schifitto G, Zhong J, Gill D et al. Lithium therapy for human immunodeficiency virus type 1-associated neurocognitive impairment. J Neurovirol 2009; 15: 176-186.

37 Schifitto G, Peterson DR, Zhong J et al. Valproic acid adjunctive therapy for HIV-associated cognitive impairment: a first report. Neurology 2006; 66: 919-921.

38 Ho DD, Rota TR, Schooley RT et al. Isolation of HTLV-III from cerebrospinal fluid and neural tissues of patients with neurologic syndromes related to the acquired immunodeficiency syndrome. N Engl J Med 1985; 313: 1493-1497.

39 Sonnerborg AB, Ehrnst AC, Bergdahl SK et al. HIV isolation from cerebrospinal fluid in relation to immunological deficiency and neurological symptoms. AIDS 1988; 2: 89-93. 
40 Rawson T, Muir D, Mackie NE et al. Factors associated with cerebrospinal fluid HIV RNA in HIV infected subjects undergoing lumbar puncture examination in a clinical setting. J Infect 2012; 65: 239-245.

41 Soulie C, Fourati S, Lambert-Niclot S et al. HIV genetic diversity between plasma and cerebrospinal fluid in patients with HIV encephalitis. AIDS 2010; 24: 2412-2414.

42 Canestri A, Lescure FX, Jaureguiberry S et al. Discordance between cerebral spinal fluid and plasma HIV replication in patients with neurological symptoms who are receiving suppressive antiretroviral therapy. Clin Infect Dis 2010; 50: 773-778.

\subsection{Chronic kidney disease}

\subsubsection{When to start antiretroviral therapy \\ 8.5.1.1 Recommendations}

- We recommend individuals with HIV-associated nephropathy (HIVAN) start ART immediately irrespective of CD4 cell count (1C).

- We recommend individuals with end-stage kidney disease who are suitable candidates for renal transplantation start ART immediately (1C).

8.5.1.2 Auditable outcome

- Proportion of individuals with HIVAN started on ART within 2 weeks of diagnosis of chronic kidney disease. 8.5.1.3 Rationale

HIV-associated nephropathy (HIVAN) is typically encountered in black individuals with advanced immunodeficiency and detectable HIV RNA levels [1-5]. The use of ART has been associated with a decline in the incidence of HIVAN in cohort studies [5], with renal histological improvement in case reports [6], and with (a trend towards) delayed progression to end-stage kidney disease in case series [2-4,7]. For those with HIVAN, ART is indicated and should be commenced as soon as possible. PLWH at risk of HIVAN: black individuals with an estimated glomerular filtration rate (eGFR) $<60 \mathrm{~mL} / \mathrm{min} /$ $1.73 \mathrm{~m}^{2}$ and/or significant proteinuria (>500-1000 mg/ $24 \mathrm{~h}$ ) should be referred to a nephrologist for a kidney biopsy. Those found to have HIVAN should be offered ART irrespective of CD4 cell count.

Immunodeficiency is a potent risk factor for chronic kidney disease (CKD) [8,9]. Although HIV replication is a risk factor for immune-complex kidney disease [2], and HIV replication, immune activation and inflammation may contribute to kidney disease progression [10,11], several antiretrovirals have been associated with eGFR decline $[8,12]$. There are currently insufficient data to suggest that HIV-positive individuals with, or at risk of CKD benefit specifically from earlier ART initiation.
Kidney transplantation is an attractive treatment modality for subjects with end-stage kidney disease. Excellent results have been achieved in those with suppressed HIV RNA levels and CD4 cell counts $>200$ cells/ $\mu \mathrm{L}$ [13-15].

\subsubsection{What to start}

8.5.2.1 Recommendations

- We recommend against the use of ARV drugs that are potentially nephrotoxic in individuals with stages 3-5 CKD if acceptable alternative ARV agents are available (GPP).

- We recommend dose adjustment of renally cleared ARV drugs in individuals with reduced renal function (GPP).

8.5.2.2 Auditable outcomes

- Proportion of individuals with CKD stages 3-5 on ARVs who commence tenofovir-DF disoproxil fumarate or atazanavir and a record of the rationale.

- Record in medical notes of calculated dose of renally cleared ARVs where eGFR $<50 \mathrm{~mL} / \mathrm{min} / 1.73 \mathrm{~m}^{2}$.

8.5.2.3 Rationale

There are no data from randomised controlled trials to inform ART decisions in individuals with CKD. Observational data suggest that kidney function improves in those with impaired kidney function following initiation of ART [16,17]. Nonetheless, an eGFR $<60 \mathrm{~mL} / \mathrm{min} /$ $1.73 \mathrm{~m}^{2}$ at baseline is a powerful predictor of kidney disease progression [18,19], and ART with nephrotoxic potential (tenofovir-DF [20-22] and atazanavir [22-24]) is probably best avoided in this population. Although lopinavir/ $\mathrm{r}$ has also been associated with an increased risk of CKD in some cohorts [12,25], there are no reports of lopinavir/r-induced renal injury in HIV-positive patients.

In individuals with impaired renal function, specific ARV drugs (all NRTIs except abacavir) may need to be dose-adjusted [26] (see also Appendix 5 Considerations for antiretrovirals in renal impairment). Impaired survival has been reported with ART prescription errors in individuals undergoing dialysis [27]. While the CockcroftGault formula has been most widely used in clinical practice to assess the need for dose reduction of renally cleared drugs in individuals with impaired renal function, recent data suggest that the eGFR (CKD-EPI equation) provides superior concordance with measured GFR in HIV-positive individuals and better performance in terms of correct dosing of tenofovir-DF and emtricitabine [28]. Hence, we recommend dose adjustment of renally cleared NRTIs in those with eGFR $<50 \mathrm{~mL} / \mathrm{min} / 1.73 \mathrm{~m}^{2}$, and of maraviroc eGFR $<80 \mathrm{~mL} / \mathrm{min} / 1.73 \mathrm{~m}^{2}$ if co-administered 
with ritonavir or cobicistat. For further information and advice, the reader should refer to the summary of product characteristics for each ARV.

\subsubsection{Need to switch}

8.5.3.1 Recommendations

- We recommend against continued use of tenofovir-DF and atazanavir in individuals with worsening renal function who have developed or are approaching CKD stages 3-5 if acceptable alternative ARV agents are available (GPP).

8.5.3.2 Auditable outcome

- Number of individuals with CKD stages 3-5 on ARVs who continue tenofovir-DF or atazanavir with eGFR $<60 \mathrm{~mL} / \mathrm{min} / 1.73 \mathrm{~m}^{2}$ and a record of the rationale.

\subsubsection{Rationale}

The risk of CKD is increased with older age, reduced estimated glomerular filtration rate (eGFR), hypertension, diabetes and with exposure to tenofovir-DF $[8,9,11,29,30]$, atazanavir $[8,30]$ and lopinavir $[8,12]$. Tenofovir-DF has been associated with reductions in eGFR [8,12,29,30], accelerated decline in eGFR [9,31], acute renal failure [32], tubulo-interstitial nephritis [22], renal tubular dysfunction [33,34] and Fanconi syndrome [20,21]. Atazanavir has been associated with reductions in eGFR (when co-administered with tenofovir-DF) [35], nephrolithiasis and tubulo-interstitial nephritis [22-24,33,36,37]. To date, no cases of lopinavir-associated kidney injury have been reported [38]. Of note, several antiretroviral drugs, including rilpivirine, ritonavir, cobicistat, raltegravir and dolutegravir inhibit tubular creatinine excretion and thereby result in modest plasma creatinine elevations and corresponding reductions in eGFR. These changes in eGFR typically manifest within 2-4 weeks, are non-progressive thereafter, not associated with haematuria, proteinuria or glycosuria, and do not reflect clinically significant kidney injury [38].

The nephrotoxic potential of tenofovir-DF appears to be increased, and the reversibility of eGFR decline following tenofovir-DF discontinuation diminished, in those with eGFR $<90 \mathrm{~mL} / \mathrm{min} / 1.73 \mathrm{~m}^{2}$ [9,26,27]. Similarly, the nephrotoxic potential of atazanavir may be increased among individuals with eGFR $<60 \mathrm{~mL} / \mathrm{min} / 1.73 \mathrm{~m}^{2}$ [23]. As CKD is a risk factor for death, AIDS, and non-AIDS events including end-stage kidney disease and cardiovascular disease events $[18,39,40]$, we recommend that the need for continued tenofovir-DF and atazanavir use is reviewed in individuals with worsening renal function who have developed or who are approaching CKD stages 3-5.

\subsubsection{References}

1 Post FA, Campbell LJ, Hamzah L et al. Predictors of renal outcome in HIV-associated nephropathy. Clin Infect Dis 2008; 46: 1282-1289.

2 Foy MC, Estrella MM, Lucas GM et al. Comparison of risk factors and outcomes in HIV immune complex kidney disease and HIV-associated nephropathy. Clin J Am Soc Nephrol 2013; 8: 1524-1532.

3 Bige N, Lanternier F, Viard JP et al. Presentation of HIVassociated nephropathy and outcome in HAART-treated patients. Nephrol Dial Transplant 2011.

4 Atta MG, Gallant JE, Rahman MH et al. Antiretroviral therapy in the treatment of HIV-associated nephropathy. Nephrol Dial Transplant 2006; 21: 2809-2813.

5 Lucas GM, Eustace JA, Sozio S et al. Highly active antiretroviral therapy and the incidence of HIV-1-associated nephropathy: a 12-year cohort study. AIDS 2004; 18: 541546.

6 Winston JA, Bruggeman LA, Ross MD et al. Nephropathy and establishment of a renal reservoir of HIV type 1 during primary infection. N Engl J Med 2001; 344: 1979-1984.

7 Szczech LA, Gupta SK, Habash R et al. The clinical epidemiology and course of the spectrum of renal diseases associated with HIV infection. Kidney Int 2004; 66: 11451152.

8 Mocroft A, Kirk 0, Reiss P et al. Estimated glomerular filtration rate, chronic kidney disease and antiretroviral drug use in HIV-positive patients. AIDS 2010; 24: 1667-1678.

9 Campbell LJ, Ibrahim F, Fisher M et al. Spectrum of chronic kidney disease in HIV-infected patients. HIV Med 2009; 10: 329-336.

10 Choi AI, Shlipak MG, Hunt PW et al. HIV-infected persons continue to lose kidney function despite successful antiretroviral therapy. AIDS 2009; 23: 2143-2149.

11 Kalayjian RC, Lau B, Mechekano RN et al. Risk factors for chronic kidney disease in a large cohort of HIV-1 infected individuals initiating antiretroviral therapy in routine care. AIDS 2012; 26: 1907-1915.

12 Ryom L, Mocroft A, Kirk 0 et al. Association between antiretroviral exposure and renal impairment among HIVpositive persons with normal baseline renal function: the D: A: D study. J Infect Dis 2013; 207: 1359-1369.

13 Stock PG, Barin B, Murphy B et al. Outcomes of kidney transplantation in HIV-infected recipients. N Engl J Med 2010; 363: 2004-2014.

14 Gathogo EN, Hamzah L, Hilton R et al. Kidney transplantation in HIV-positive adults: the UK experience. International journal of STD \& AIDS 2014; 25: 57-66.

15 Gathogo E, Jose S, Jones R et al. End-Stage Kidney Disease and Kidney Transplantation in HIV-Positive Patients: An Observational Cohort Study. J Acquir Immune Defic Syndr 2014; 67: 177-180. 
16 Reid A, Stohr W, Walker AS et al. Severe renal dysfunction and risk factors associated with renal impairment in HIVinfected adults in Africa initiating antiretroviral therapy. Clin Infect Dis 2008; 46: 1271-1281.

17 Kalayjian RC, Franceschini N, Gupta SK et al. Suppression of HIV-1 replication by antiretroviral therapy improves renal function in persons with low CD4 cell counts and chronic kidney disease. AIDS 2008; 22: 481-487.

18 Ibrahim F, Hamzah L, Jones R et al. Baseline Kidney function as predictor of mortality and kidney disease progression in HIV-positive patients. Am J Kidney Dis 2012; 60: 539-547.

19 Ryom L, Mocroft A, Kirk 0 et al. Predictors of advanced chronic kidney disease and end-stage renal disease in HIVpositive persons. AIDS 2014; 28: 187-199.

20 Woodward CL, Hall AM, Williams IG et al. Tenofovirassociated renal and bone toxicity. HIV Med 2009; 10: 482487.

21 Zimmermann AE, Pizzoferrato T, Bedford J et al. Tenofovirassociated acute and chronic kidney disease: a case of multiple drug interactions. Clin Infect Dis 2006; 42: 283 290.

22 Schmid S, Opravil M, Moddel M et al. Acute interstitial nephritis of HIV-positive patients under atazanavir and tenofovir therapy in a retrospective analysis of kidney biopsies. Virchows Arch 2007; 450: 665-670.

23 Rockwood N, Mandalia S, Bower M et al. Ritonavir-boosted atazanavir exposure is associated with an increased rate of renal stones compared with efavirenz, ritonavir-boosted lopinavir and ritonavir-boosted darunavir. AIDS 2011; 25: 1671-1673.

24 Couzigou C, Daudon M, Meynard JL et al. Urolithiasis in HIV-positive patients treated with atazanavir. Clin Infect Dis 2007; 45: e105-108.

25 Mocroft A, Lundgren JD, Ross M et al. Development and validation of a risk score for chronic kidney disease in HIV infection using prospective cohort data from the D:A: D study. PLoS Med 2015; 12: e1001809.

26 Brennan A, Evans D, Maskew M et al. Relationship between renal dysfunction, nephrotoxicity and death among HIV adults on tenofovir. AIDS 2011; 25: 1603-1609.

27 Jose S, Hamzah L, Campbell LJ et al. Incomplete reversibility of estimated glomerular filtration rate decline following tenofovir disoproxil fumarate exposure. J Infect Dis 2014; 210: 363-373.

28 Okparavero AA, Tighiouart H, Krishnasami Z et al. Use of glomerular filtration rate estimating equations for drug dosing in HIV-positive patients. Antivir Ther 2013; 18:

793-802.

29 Scherzer R, Estrella M, Li Y et al. Association of tenofovir exposure with kidney disease risk in HIV infection. AIDS 2012; 26: 867-875.

30 Rasch MG, Engsig FN, Feldt-Rasmussen B et al. Renal function and incidence of chronic kidney disease in HIV patients: a Danish cohort study. Scand J Infect Dis 2012; 44: 689-696.

31 Nishijima T, Kawasaki Y, Tanaka N et al. Long-term exposure to tenofovir continuously decrease renal function in HIV-1-infected patients with low body weight: results from 10 years of follow-up. AIDS 2014; 28: 1903-1910.

32 Herlitz LC, Mohan S, Stokes MB et al. Tenofovir nephrotoxicity: acute tubular necrosis with distinctive clinical, pathological, and mitochondrial abnormalities. Kidney Int 2010; 78: 1171-1177.

33 Dauchy FA, Lawson-Ayayi S, de La Faille R et al. Increased risk of abnormal proximal renal tubular function with HIV infection and antiretroviral therapy. Kidney Int 2011; 80: 302-309.

34 Labarga P, Barreiro P, Martin-Carbonero L et al. Kidney tubular abnormalities in the absence of impaired glomerular function in HIV patients treated with tenofovir. AIDS 2009; 23: 689-696.

35 Daar ES, Tierney C, Fischl MA et al. Atazanavir plus ritonavir or efavirenz as part of a 3-drug regimen for initial treatment of HIV-1. Ann Intern Med 2011; 154: 445-456.

36 Rakotondravelo S, Poinsignon Y, Borsa-Lebas F et al. Complicated atazanavir-associated cholelithiasis: a report of 14 cases. Clin Infect Dis 2012; 55: 1270-1272.

37 Hamada Y, Nishijima T, Watanabe K et al. High incidence of renal stones among HIV-infected patients on ritonavirboosted atazanavir than in those receiving other protease inhibitor-containing antiretroviral therapy. Clin Infect Dis 2012; 55: 1262-1269.

38 Yombi JC, Pozniak A, Boffito M et al. Antiretrovirals and the kidney in current clinical practice: renal pharmacokinetics, alterations of renal function and renal toxicity. AIDS 2014; 28: 621-632.

39 Choi AI, Li Y, Deeks SG et al. Association between kidney function and albuminuria with cardiovascular events in HIV-infected persons. Circulation 2010; 121: 651-658.

40 Mocroft A, Ryom L, Begovac J et al. Deteriorating renal function and clinical outcomes in HIV-positive persons. AIDS 2014; 28: 727-737. 


\subsection{Cardiovascular disease}

\subsubsection{Introduction}

Cardiovascular disease (CVD) is a significant contributor to the excess risk of non-AIDS disease and death in HIVpositive populations [1-12] but there are encouraging recent data that rates of ischaemic stroke [10] CVDrelated deaths may be falling over time [13].

The heightened risk of CVD has been attributed to intersecting epidemics of smoking and inactivity, an ageing HIV-positive cohort, increased prevalence of surrogate markers of CVD (such as dyslipidaemia), HIV viraemia, immune dysfunction and the pro-inflammatory state associated with HIV infection. HIV-related factors may be only partially mitigated by ART and may be exacerbated by effects not fully explained by conventional CVD risk factors. Therefore it would be wrong to presume that interventions proven to reduce CVD risk in the general population will have the same magnitude of effect in HIV-positive individuals [14].

\subsubsection{Definition and assessment of cardiovascular disease risk}

8.6.2.1 Recommendation

- We suggest that the coronary heart disease (CHD) risk of HIV-positive adults of white ethnicity is estimated as per the BHIVA guidelines for the routine investigation and monitoring of adult HIV-1-infected individuals (www.bhiva.org/monitoring-guidelines.aspx) (2C).

8.6.2.2 Rationale

For the purposes of these guidelines, individuals with an elevated CVD risk are those with established atherosclerotic CVD; diabetes mellitus type 1 over the age of 40 years; an estimated glomerular filtration rate (eGFR) $<60 \mathrm{~mL} / \mathrm{min} / 1.73 \mathrm{~m}^{2}$ and/or albuminuria; familial hypercholesterolaemia; a high calculated CVD risk [15].

NICE recommends the QRISK2 calculator for the English population [15] but this may not be appropriate for HIV-positive people. Myocardial infarction (MI) rates in HIV-positive people parallel those predicted by the Framingham risk equation [16] but in US males it underestimated risk of MI by 50\% [9] while in D:A:D it appeared to overestimate risk in never smokers but underestimate it in women, diabetics and ex-smokers [17]. Although absolute risk of CVD is lower in HIV-positive women than men, the risk relative to HIV-negative women is significantly higher $[6,18,19]$. The D:A:D calculator more accurately predicted CHD risk among European/Australian HIV-positive populations, defining 5-year risk as low $(<1 \%)$, moderate $(1-5 \%)$, high $(5-10 \%)$ and very high $(>10 \%)$ risk of CHD over a 5-year period [18]. However, it has not been externally validated, it lacked power to predict risk beyond 5 years and was underpowered for women and those of non-white ethnicity [20]. There is no HIV-specific CVD risk calculator for those of non-white ethnicity; one approach might be to use the QRISK2 equation and apply a correction for HIV status of 1.6 [21] (2D).

Whether HCV co-infection is a CVD risk is unclear; with studies reporting a significant association for acute coronary events [22] and stroke [23], a non-significant trend for MI [23], or no association [24].

\subsubsection{Issues related to antiretroviral therapy}

Two randomised controlled trials have included CVD endpoints: SMART and START; although neither were powered specifically for a CVD endpoint. In the SMART trial, fewer major CVD events were observed in the viral suppression arm but the difference was not statistically significant [25]. In a post hoc analysis, neither viral load or CD4 cell count was significantly associated with CVD events [26]. In START, rates of CVD events were similar in both arms; but this was a young population with a low CVD risk (median 10-year Framingham risk 1.9\%) [27]. START does not answer the question of whether HAART affects CVD incidence in those at high CVD risk.

A meta-analysis showed an increased risk of CVD events in those on ARVs compared to treatment-naïve individuals, but the source studies pre-dated currently available PIs [21]. Several observational studies have examined changes in rate of cardiovascular events in HIV-positive populations but there are no clear protective effects for those on ART, those with a nadir or current CD4 cell count $>350$ cells/ $\mu \mathrm{L}$ or suppressed viraemia (Table 8.6.1).

Carotid intima-media thickness (cIMT) may be increased in HIV-positive adults [28-30] although this has not been a consistent finding [31]. Non-calcified coronary arterial plaques were more prevalent on CT in HIV-positive participants in the MACS cohort than among controls and severe coronary arterial stenosis was associated with lower nadir CD4 cell count and longer duration of ART, but not current viral load or CD4 cell count [32].

\subsubsection{What to start}

\subsubsection{Recommendations}

In individuals with a high CVD risk:

- We recommend use of alternatives to fosamprenavir/r (1C) and lopinavir/r (1C)

- We suggest that atazanavir/ $\mathrm{r}$ is the preferred PI (2C).

- We suggest avoiding abacavir (2C) and maraviroc if an acceptable alternative is available.

- First-line ARV therapy with tenofovir-DF plus (emtricitabine or lamivudine) with dolutegravir or raltegravir or rilpivirine (if viral load $<100,000$ copies/mL) are preferred first line regimens (GPP). 
Table 8.6.1 Summary of observational studies reporting CVD risk independently associated with low CD4 cell count (cells/ $\mu \mathrm{L}$ ), elevated plasma viral load (copies/mL) or ARV exposure.

\begin{tabular}{|c|c|c|c|c|c|c|c|c|c|}
\hline $\begin{array}{l}\text { Type of } \\
\text { study }\end{array}$ & Year & Outcome & $\begin{array}{l}\text { First CD4 cell } \\
\text { count (cells/ } \\
\mu \mathrm{L})\end{array}$ & $\begin{array}{l}\text { Nadir CD4 (cells/ } \\
\mu \mathrm{L})\end{array}$ & $\begin{array}{l}\text { Recent CD4 } \\
\text { cell count } \\
\text { (cells/ } \mu \mathrm{L} \text { ) }\end{array}$ & $\begin{array}{l}\text { First viral } \\
\text { load } \\
\text { (copies/mL) }\end{array}$ & $\begin{array}{l}\text { Current VL } \\
\text { (copies } / \mathrm{mL} \text { ) }\end{array}$ & ARVs & Ref \\
\hline Co & $\begin{array}{r}1993- \\
2001\end{array}$ & Composite $^{6}$ & - & - & - & - & - & $\leftrightarrow$ & [35] \\
\hline Co & $\begin{array}{r}1999- \\
2002\end{array}$ & MI & No & No & - & No & - & $\uparrow$ & {$[58]$} \\
\hline Co & $\begin{array}{r}1997- \\
2003\end{array}$ & Death & - & - & - & - & - & $\uparrow$ & [59] \\
\hline Co & $\begin{array}{r}1996- \\
2004\end{array}$ & Death & - & - & - & - & - & $\downarrow$ & {$[60]$} \\
\hline Co & $\begin{array}{r}2000- \\
2004\end{array}$ & Admission/death & - & - & No & & & $\leftrightarrow$ & [61] \\
\hline $\mathrm{CC}$ & $\begin{array}{r}2000- \\
2006\end{array}$ & $\mathrm{Ml}$ & - & Yes $^{5}$ & No ${ }^{5}$ & - & Yes $^{5}$ & - & [39] \\
\hline Co & $\begin{array}{r}1998- \\
2008\end{array}$ & MI & - & No & $<200$ & No & No & $\leftrightarrow$ & [62] \\
\hline RCT & $\begin{array}{r}2000- \\
2008\end{array}$ & Composite $^{1}$ & - & - & No & - & - & - & [63] \\
\hline Co & $\begin{array}{r}2000- \\
2008\end{array}$ & $\begin{array}{l}\text { MI or } \\
\quad \text { Composite }\end{array}$ & - & - & No & - & No & - & [17] \\
\hline Co & $\begin{array}{r}2002- \\
2009\end{array}$ & Composite $^{7}$ & $<350$ & No & - & No & - & $\leftrightarrow$ & [64] \\
\hline $\mathrm{CC}$ & $\begin{array}{r}2002- \\
2009\end{array}$ & Composite $^{7}$ & No & No & $\begin{array}{l}<350 \text { \&t } 350- \\
500\end{array}$ & No & - & $\leftrightarrow$ & [64] \\
\hline Co & $\begin{array}{r}2003- \\
2009\end{array}$ & MI & No & - & $<200$ & No & $>500$ & - & [9] \\
\hline Co & $\begin{array}{r}2003- \\
2010\end{array}$ & Composite $^{2}$ & - & - & No & - & $\begin{array}{l}\text { Prolonged } \\
\text { rebound } \\
>400\end{array}$ & - & [65] \\
\hline Co & $\begin{array}{r}1996- \\
2011\end{array}$ & Ischaemic stroke & No & No & $<200$ & - & No & $\begin{array}{l}\text { No } \\
\text { effect }\end{array}$ & [10] \\
\hline Co & $\begin{array}{r}1996- \\
2011\end{array}$ & $\mathrm{Ml}$ & - & $<200$ \&t $200-500^{3}$ & No & - & No & $\begin{array}{l}\text { No } \\
\text { effect }\end{array}$ & [66] \\
\hline
\end{tabular}

Co: cohort study; CC: case-control study; RCT: randomised controlled trial.

${ }^{1} \mathrm{MI}$, stroke, and invasive coronary procedures.

${ }^{2} \mathrm{MI}$, stroke, and invasive coronary procedures, other fatal heart/vascular events and sudden death.

${ }^{3}$ Relative to HIV-negative population; the risk for 350-500 cells was not reported.

${ }^{4} \mathrm{MI}$, stroke, and invasive coronary procedures, other fatal heart/vascular events, sudden death and carotid endarterectomy.

${ }^{5}$ Lower nadir increased risk on univariate analysis only.

${ }^{6}$ Admissions with MI, angina, stroke, subarachnoid haemorrhage, transient cerebral ischaemia, invasive coronary procedures, other fatal cardiovascular events.

${ }^{7} \mathrm{MI}$, stroke, coronary artery disease, angina and peripheral arterial disease.

- Adverse effects on lipid parameters should be considered when selecting ARVs (GPP).

8.6.4.2 Rationale

No randomised controlled trial has been powered for the CVD risk associated with the use of individual ARVs and a history of CVD may be an exclusion criterion. A systematic review found a harmful association with recent PI exposure and cumulative increased risk per year of exposure to lopinavir/r and indinavir [33]. Early studies of PI exposure and risk of MI gave conflicting results, some reporting an increased risk [3,34] while others did not $[2,35,36]$. The D:A:D cohort, with longer follow-up, reported an increasing risk of MI with years of PI exposure (independent of measured metabolic effects) [37]. Cumulative exposure to indinavir and lopinavir/r were associated with increasing risk of MI [38]. Case-control studies reported similar associations for PIs [39], lopina$\mathrm{vir} / \mathrm{r}[8,40]$ and fosamprenavir/r [40] although not all controlled for established risk factors [8]. Others found no association between PI use and cerebrovascular events [10,11]. No association has been reported between use of atazanavir/r and risk of MI [41] but there were insufficient data to include darunavir/r in this analysis. Preliminary data showed slower progression of cIMT in subjects on atazanavir/r versus darunavir/r (raltegravir was intermediate) [42] but whether this equates to a reduction in CVD risk is unclear.

Four papers analysing pooled data from clinical trials of abacavir [43-45] found no excess risk of MI for abacavir, but there was significant overlap in the source 
trials for these analyses. In contrast a post hoc analysis of the SMART study did find an increased risk (although use of abacavir was not randomised) [46]. A systematic review of CVD events associated with ARVs did find a harmful association with recent abacavir exposure [33]. Two cohorts found a strong association between recent abacavir use and MI $[38,47,48]$ while others did not [4951]; all were limited in their ability to adjust for presence of CVD risk factors. One case-control study, which did not adjust for important CVD risk factors, did find an elevated risk of MI associated with abacavir use [8] but others did not $[38,52]$. Cerebrovascular events were more common in individuals exposed to abacavir in two cohort studies [11,51] while another found a protective effect [49]. These divergent findings may be explained by unmeasured confounding, detectable viraemia (in studies of people commencing ARVs), younger participants in trials than in cohorts, lack of power in trials due to smaller number of MI events and differences in case ascertainment $[14,53]$. In view of the uncertainty about the safety of abacavir in individuals with a high CVD risk, we suggest the use of alternative agents where possible.

In the MOTIVATE studies for treatment-experienced individuals, coronary artery disease events were only reported in the maraviroc arm (11 in 609 patient years versus 0 in 111 patient years in controls). No such signal was found in the MERIT study for treatment-naïve individuals. Maraviroc has also been associated with postural hypotension when used at higher than recommended doses in healthy volunteers; people with a history of postural hypotension, renal impairment or taking antihypertensive agents may be at increased risk [54]. In view of the limited data available, caution should be exercised in the use of maraviroc in individuals with a high CVD risk and use of alternative agents, where possible, considered.

For those with a high CVD risk, the newer agents dolutegravir, raltegravir and rilpivirine offer advantages including their lack of effect on plasma lipids (Table 8.6.2) and absence of interactions with statins. However, as yet, there is insufficient experience in their use to exclude an unexpected adverse CVD risk.

The effects on plasma lipids reported in randomised controlled trials of first line antiretrovirals are shown in Table 8.6.2. The studies were not usually powered for these comparisons and it is not possible to make crossstudy comparisons are as they differed in their populations, baseline lipids and length of follow up. Low highdensity lipoprotein (HDL) cholesterol is a common finding in untreated HIV infection and may persist on ART [55]. Several studies report increases in both total and HDL cholesterol and no effect on total cholesterol (TC)/HDL ratio. However, HDL rises due to ARVs may not have the same cardioprotective effect as untreated HDLs as they may be larger, less stable and bind less readily to hepatocyte receptors relative to HIV-negative normolipaemic subject [56]. Moreover other pharmacological interventions to increase HDL in HIV-negative individuals on statins have failed to reduce CVD risk [57]. Therefore, favourable changes in HDL on treatment should be interpreted cautiously.

Table 8.6.2 Increases in plasma lipids in randomised controlled trials of first-line antiretroviral exposure. Where a significant difference was reported, the mean is shown in $\mathrm{mmol} / \mathrm{L}$.

\begin{tabular}{|c|c|c|c|c|c|c|c|c|}
\hline Class & Drug & Versus & TC & LDL & HDL & $\mathrm{TC} / \mathrm{HDL}$ & TGs & Ref \\
\hline NRTls & АBC/3ТC & TDF/FTC & 0.88 vs. 0.49 & 0.44 vs. 0.16 & 0.31 vs. 0.23 & NS & NS & [67] \\
\hline \multirow[t]{5}{*}{ NNRTIs } & EFV $600 \mathrm{mg}$ & EFV $400 \mathrm{mg}$ & NS & NS & NS & - & NS & [68] \\
\hline & NVP & EFV & NS & NS & 0.3 vs. 0.2 & 3.7 vs. 4.2 & 0.0 vs. 0.2 & [69] \\
\hline & RPV & Baseline & NS & NS & - & NS & NS & [70] \\
\hline & RPV & EFV & 0.03 vs. 0.63 & -0.04 vs. 0.31 & 0.07 vs. 0.24 & NS & -0.01 vs. 0.16 & [70] \\
\hline & ETV & EFV & 0.4 vs. 1.0 & 0.2 vs. 0.6 & 0.1 vs. 0.3 & NS & - & [71] \\
\hline \multirow[t]{6}{*}{ Pls } & $\mathrm{ATV} / \mathrm{r}$ & EFV & 0.25 vs. 0.49 & 0.18 vs. 0.54 & 0.10 vs. 0.2 & -0.1 vs. 0.3 & NS & [72] \\
\hline & $\mathrm{ATV} / \mathrm{r}$ & NVP & 0.51 vs. 0.63 & 0.27 vs. 0.39 & 0.10 vs. 0.25 & 0.13 vs. -0.24 & 0.31 vs. 0.00 & [73] \\
\hline & $\mathrm{ATV} / \mathrm{r}$ & RAL & ATV/r 0.31 higher & ATV/r 0.18 higher & NS & - & ATV/r 0.32 higher & [74] \\
\hline & $\mathrm{DRV} / \mathrm{r}$ & $\mathrm{ATV} / \mathrm{r}$ & NS & NS & NS & - & NS & [74] \\
\hline & $\mathrm{DRV} / \mathrm{r}$ & RAL & DRV/r 0.28 higher & DRV/r 0.18 higher & NS & - & DRV/r 0.27 higher & [74] \\
\hline & $L P V / r$ & $\mathrm{ATV} / \mathrm{r}$ & 0.93 vs. 0.52 & NS & NS & - & 0.62 vs. 0.16 & [75] \\
\hline \multirow[t]{6}{*}{ INI } & RAL & Baseline & NS & NS & NS & & NS & [76] \\
\hline & RAL & Baseline & NS & NS & 0.16 & & NS & [74] \\
\hline & RAL & EFV & 0.26 vs. 0.85 & 0.15 vs. 0.42 & 0.11 vs. 0.26 & NS & -0.03 vs. 0.42 & [77] \\
\hline & $\mathrm{ELV} / \mathrm{c}$ & EFV & 0.41 vs. 0.52 & 0.31 vs. 0.47 & 0.16 vs. 0.23 & NS & NS & [78] \\
\hline & $\mathrm{ELV} / \mathrm{c}$ & $\mathrm{ATV} / \mathrm{r}$ & NS & NS & NS & NS & NS & [79] \\
\hline & DTG & Baseline & NS & NS & NS & NS & NS & [76] \\
\hline CCR5 & MVC & EFV & 0.05 vs. 0.93 & -0.23 vs. 0.54 & 0.18 vs. 0.35 & - & $-0.10 \vee 0.24$ & [80] \\
\hline
\end{tabular}

NS: difference not statistically significant; ATV/r: atazanavir/ritonavir; DRV/r: darunavir/ritonavir; EFV: efavirenz; ELV/c: elvitegravir/cobicistat; ETV: etravirine; LPV/r: lopinavir/ritonavir; NVP: nevirapine; RAL: raltegravir; RPV: rilpivirine; RPV/TDF/FTC: rilpivirine/tenofovir-DF/emtricitabine. 
Table 8.6.3 Changes in plasma lipids reported in trials of switching antiretrovirals. Where a significant difference was reported, the mean is shown in $\mathrm{mmol} / \mathrm{L}$

\begin{tabular}{|c|c|c|c|c|c|c|c|}
\hline Class & Drug & $\mathrm{TC}$ & LDL & HDL & $\mathrm{TC} / \mathrm{HDL}$ & TGs & Ref \\
\hline NRTIs & Kivexa to Truvada ${ }^{1}$ & -0.47 & -0.23 & NS & NS & NS & [81] \\
\hline \multirow[t]{6}{*}{ NNRTIs } & EFV to RAL & -0.36 & NS & NS & - & NS & [82] \\
\hline & EFV to ELV/c & -0.21 & -0.20 & NS & NS & NS & [83] \\
\hline & EFV to NVP & NS & -0.34 & NS & - & NS & [84] \\
\hline & EFV to RPV & -0.44 & -0.21 & NS & NS & -0.29 & [85] \\
\hline & $\mathrm{Pl} / \mathrm{r}+2 \mathrm{NRTI}$ to $\mathrm{RPV} / \mathrm{TDF} / \mathrm{FTC}$ & -0.62 & -0.41 & -0.07 & -0.35 & -0.63 & [86] \\
\hline & EFV to ETV & -0.64 & -0.58 & NS & NR & NS & [87] \\
\hline \multirow[t]{7}{*}{ Pls } & $\mathrm{ATV} / \mathrm{r}$ to $\mathrm{ATV}^{2}$ & -0.34 & NS & NS & -0.24 & -0.36 & [88] \\
\hline & $\mathrm{ATV} / \mathrm{r}$ to $\mathrm{RAL}$ & NS & - & - & - & NS & [89] \\
\hline & LPV/r to ATV/r & -0.39 & NS & NS & - & -0.55 & [90] \\
\hline & $\mathrm{LPV} / \mathrm{r}$ to $\mathrm{RAL}$ & -0.44 & - & - & - & -0.49 & [89] \\
\hline & $\mathrm{LPV} / \mathrm{r}$ to $\mathrm{RAL}^{3}$ & -0.8 & NS & - & - & -0.90 & [91] \\
\hline & $\mathrm{ATV} / \mathrm{r}$ to $\mathrm{ELV} / \mathrm{c}$ & NS & NS & NS & NS & -0.42 & [92] \\
\hline & $\mathrm{DRV} / \mathrm{r}$ to $\mathrm{ELV} / \mathrm{c}$ & NS & NS & NS & NS & NS & [92] \\
\hline
\end{tabular}

NS: difference not statistically significant; ABC/3TC: abacavir/lamivudine; ATV/r: atazanavir/ritonavir; DRV/r: darunavir/ritonavir; DTG: dolutegravir; EFV: efavirenz; ELV/c: elvitegravir/cobicistat; ETV: etravirine; LPV/r: lopinavir/ritonavir; MVC: maraviroc; NVP: nevirapine; RAL: raltegravir; RPV: rilpivirine; TDF/FTC: tenofovir-DF/emtricitabine.

${ }^{1}$ With PI/r; ${ }^{2}$ Atazanavir plus abacavir/lamivudine backbone; ${ }^{3}$ Powered for a change in lipids.

\subsubsection{Modification of CVD risk factors}

8.6.5.1 Recommendations

In patients with a high CVD risk:

- We recommend that traditional modifiable risk factors should be minimised; smoking cessation is of critical importance (1A).

- We suggest that that this should include switching ARVs to those with a more favourable metabolic profile but only where there is minimal risk of treatment failure (2C).

8.6.5.2 Rationale

There is no clinical trial evidence that interventions to modify plasma lipids will reduce CVD risk in the context of HIV disease. However, there is good evidence from the general population that reducing total and LDL cholesterol reduces CVD risk. Switching ARVs in order to contribute to this reduction may therefore be beneficial. Switch studies that have reported lipid outcomes are shown in Table 8.6.3 and the same caveats apply as to Table 8.6.2 (above). In addition, the data are expressed in terms of the mean; whether the effect size differed in those with the most adverse lipid profiles is not reported. A switch should only be considered if the risk of treatment failure is minimal.

\subsubsection{References}

1 Currier JS, Taylor A, Boyd F et al. Coronary heart disease in HIV-infected individuals. J Acquir Immune Defic Syndr 2003; 33: 506-512.

2 Klein D, Hurley LB, Quesenberry CP Jr, Sidney S. Do protease inhibitors increase the risk for coronary heart disease in patients with HIV-1 infection? J Acquir Immune Defic Syndr 2002; 30: 471-477.
3 Mary-Krause M, Cotte L, Simon A et al. Increased risk of myocardial infarction with duration of protease inhibitor therapy in HIV-infected men. AIDS 2003; 17: 2479-2486.

4 Mocroft A, Reiss P, Gasiorowski J et al. Serious fatal and nonfatal non-AIDS-defining illnesses in Europe. J Acquir Immune Defic Syndr 2010; 55: 262-270.

5 Smith C, Sabin CA, Lundgren JD et al. Factors associated with specific causes of death amongst HIV-positive individuals in the D:A: D Study. AIDS 2010; 24: 15371548.

6 Triant VA, Lee H, Hadigan C, Grinspoon SK. Increased acute myocardial infarction rates and cardiovascular risk factors among patients with human immunodeficiency virus disease. J Clin Endocrinol Metab 2007; 92: 2506-2512.

7 Chow FC, Regan S, Feske S et al. Comparison of ischemic stroke incidence in HIV-infected and non-HIV-infected patients in a US health care system. J Acquir Immune Defic Syndr 2012; 60: 351-358.

8 Durand M, Sheehy 0, Baril JG et al. Association between HIV infection, antiretroviral therapy, and risk of acute myocardial infarction: a cohort and nested case-control study using Quebec's public health insurance database. $J$ Acquir Immune Defic Syndr 2011; 57: 245-253.

9 Freiberg MS, Chang CC, Kuller LH et al. HIV infection and the risk of acute myocardial infarction. JAMA Intern Med 2013; 173: 614-622.

10 Marcus JL, Leyden WA, Chao CR et al. HIV infection and incidence of ischemic stroke. AIDS 2014; 28: 1911-1919.

11 Rasmussen LD, Engsig FN, Christensen H et al. Risk of cerebrovascular events in persons with and without HIV: a Danish nationwide population-based cohort study. AIDS 2011; 25: 1637-1646. 
12 Tseng ZH, Secemsky EA, Dowdy D et al. Sudden cardiac death in patients with human immunodeficiency virus infection. J Am Coll Cardiol 2012; 59: 1891-1896.

13 Smith CJ, Ryom L, Weber R et al. Trends in underlying causes of death in people with HIV from 1999 to 2011 (D:A:D): a multicohort collaboration. Lancet 2014; 384: 241-248.

14 Mallon PW. Impact of nucleoside reverse transcriptase inhibitors on coronary heart disease. Rev Cardiovasc Med 2014; 15 (Suppl 1): S21-29.

15 National Institue for Health and Clinical Excellence. Lipid modification: cardiovascular risk assessment and the modification of blood lipids for the primary and secondary prevention of cardiovascular disease. NICE CG181. 2014. Available at: http://www.nice.org.uk/guidance/cg181 (accessed September 2015).

16 Law MG, Friis-Moller N, El-Sadr WM et al. The use of the Framingham equation to predict myocardial infarctions in HIV-infected patients: comparison with observed events in the D:A: D Study. HIV Med 2006; 7: 218-230.

17 Friis-Moller N, Thiebaut R, Reiss $\mathrm{P}$ et al. Predicting the risk of cardiovascular disease in HIV-infected patients: the data collection on adverse effects of anti-HIV drugs study. Eur $J$ Cardiovasc Prev Rehabil 2010; 17: 491-501.

18 Lang S, Mary-Krause M, Cotte L et al. Increased risk of myocardial infarction in HIV-infected patients in France, relative to the general population. AIDS 2010; 24: 12281230.

19 Obel N, Thomsen HF, Kronborg G et al. Ischemic heart disease in HIV-infected and HIV-uninfected individuals: a population-based cohort study. Clin Infect Dis 2007; 44: 1625-1631.

20 D'Agostino RB Sr. Cardiovascular risk estimation in 2012: lessons learned and applicability to the HIV population. $J$ Infect Dis 2012; 205 (Suppl 3): S362-367.

21 Islam FM, Wu J, Jansson J, Wilson DP. Relative risk of cardiovascular disease among people living with HIV: a systematic review and meta-analysis. HIV Med 2012; 13: 453-468.

22 Freiberg MS, Chang CC, Skanderson M et al. The risk of incident coronary heart disease among veterans with and without HIV and hepatitis C. Circ Cardiovasc Qual Outcomes 2011; 4: 425-432.

23 Bedimo R, Westfall A0, Mugavero M et al. Hepatitis C virus coinfection and the risk of cardiovascular disease among HIV-infected patients. HIV Med 2010; 11: 462468.

24 Weber R, Sabin C, Reiss P et al. HBV or HCV coinfections and risk of myocardial infarction in HIV-infected individuals: the D:A: D Cohort Study. Antivir Ther 2010; 15: 1077-1086.
25 El-Sadr WM, Lundgren J, Neaton JD et al. CD4 + countguided interruption of antiretroviral treatment. $N$ Engl $J$ Med 2006; 355: 2283-2296.

26 Phillips AN, Carr A, Neuhaus J et al. Interruption of antiretroviral therapy and risk of cardiovascular disease in persons with HIV-1 infection: exploratory analyses from the SMART trial. Antivir Ther 2008; 13: 177-187.

27 Lundgren JD, Babiker AG, Gordin F et al. Initiation of Antiretroviral Therapy in Early Asymptomatic HIV Infection. $N$ Engl J Med 2015; 373: 795-807.

28 Grunfeld C, Delaney JA, Wanke C et al. Preclinical atherosclerosis due to HIV infection: carotid intima-medial thickness measurements from the FRAM study. AIDS 2009; 23: 1841-1849.

29 Hsue PY, Lo JC, Franklin A et al. Progression of atherosclerosis as assessed by carotid intima-media thickness in patients with HIV infection. Circulation 2004; 109: 1603-1608.

30 Hsue PY, Scherzer R, Hunt PW et al. Carotid intima-media thickness progression in HIV-infected adults occurs preferentially at the carotid bifurcation and is predicted by inflammation. J Am Heart Assoc 2012; 1.

31 Currier JS, Kendall MA, Henry WK et al. Progression of carotid artery intima-media thickening in HIV-infected and uninfected adults. AIDS 2007; 21: 1137-1145.

32 Post WS, Budoff M, Kingsley L et al. Associations between HIV infection and subclinical coronary atherosclerosis. Ann Intern Med 2014; 160: 458-467.

33 Bavinger C, Bendavid E, Niehaus K et al. Risk of cardiovascular disease from antiretroviral therapy for HIV: a systematic review. PLoS One 2013; 8: e59551.

34 Holmberg SD, Moorman AC, Williamson JM et al. Protease inhibitors and cardiovascular outcomes in patients with HIV-1. Lancet 2002; 360: 1747-1748.

35 Bozzette SA, Ake CF, Tam HK et al. Cardiovascular and cerebrovascular events in patients treated for human immunodeficiency virus infection. $N$ Engl J Med 2003; 348: 702-710.

36 Iloeje UH, Yuan Y, L'Italien G et al. Protease inhibitor exposure and increased risk of cardiovascular disease in HIV-infected patients. HIV Med 2005; 6: 37-44.

37 Friis-Moller N, Reiss P, Sabin CA et al. Class of antiretroviral drugs and the risk of myocardial infarction. $N$ Engl J Med 2007; 356: 1723-1735.

38 Worm SW, Sabin C, Weber R et al. Risk of myocardial infarction in patients with HIV infection exposed to specific individual antiretroviral drugs from the 3 major drug classes: the data collection on adverse events of anti-HIV drugs (D:A:D) study. J Infect Dis 2010; 201: 318-330. 
39 Lang S, Mary-Krause M, Simon A et al. HIV replication and immune status are independent predictors of the risk of myocardial infarction in HIV-infected individuals. Clin Infect Dis 2012; 55: 600-607.

40 Lang S, Mary-Krause M, Cotte L et al. Impact of individual antiretroviral drugs on the risk of myocardial infarction in human immunodeficiency virus-infected patients: a casecontrol study nested within the French Hospital Database on HIV ANRS cohort C04. Arch Intern Med 2010; 170: 1228 1238.

41 Monforte A, Reiss P, Ryom L et al. Atazanavir is not associated with an increased risk of cardio- or cerebrovascular disease events. AIDS 2013; 27: 407-415.

42 Stein $\mathrm{JH}$, Hodis $\mathrm{H}$, Brown $\mathrm{T}$ et al. Prospective randomised clinical trial of the effects of three modern antiretroviral therapies on carotid intima-media thickness in HIV-infected individuals (AIDS Clinical Trials Group Study A5260S). $J$ Am Coll Cardiol 2014; 63 (Suppl 1): A1322.

43 Brothers $\mathrm{CH}$, Hernandez JE, Cutrell AG et al. Risk of myocardial infarction and abacavir therapy: no increased risk across 52 GlaxoSmithKline-sponsored clinical trials in adult subjects. J Acquir Immune Defic Syndr 2009; 51: 2028

44 Ribaudo HJ, Benson CA, Zheng Y et al. No risk of myocardial infarction associated with initial antiretroviral treatment containing abacavir: short and long-term results from ACTG A5001/ALLRT. Clin Infect Dis 2011; 52: 929940.

45 Cruciani M, Zanichelli V, Serpelloni G et al. Abacavir use and cardiovascular disease events: a meta-analysis of published and unpublished data. AIDS 2011; 25: 19932004.

46 Sabin CA, Worm SW, Weber R et al. Use of nucleoside reverse transcriptase inhibitors and risk of myocardial infarction in HIV-infected patients. AIDS 2008; 22: F17-24.

47 Obel N, Farkas DK, Kronborg G et al. Abacavir and risk of myocardial infarction in HIV-infected patients on highly active antiretroviral therapy: a population-based nationwide cohort study. HIV Med 2010; 11: 130-136.

48 Sabin C, Reiss P, Ryom L et al. Is there continued evidence for an association between abacavir and myocardial infarction risk? Conference on Retroviruses and Opportunistic Infections. March 2014. Boston, MA, USA.

49 Bedimo RJ, Westfall A0, Drechsler H et al. Abacavir use and risk of acute myocardial infarction and cerebrovascular events in the highly active antiretroviral therapy era. Clin Infect Dis 2011; 53: 84-91.

50 Brouwer ES, Napravnik S, Eron JJ Jr et al. Effects of combination antiretroviral therapies on the risk of myocardial infarction among HIV patients. Epidemiology

2014; 25: 406-417.

51 Choi AI, Vittinghoff E, Deeks SG et al. Cardiovascular risks associated with abacavir and tenofovir exposure in HIVinfected persons. AIDS 2011; 25: 1289-1298.

52 Rotger M, Glass TR, Junier T et al. Contribution of genetic background, traditional risk factors, and HIV-related factors to coronary artery disease events in HIV-positive persons. Clin Infect Dis 2013; 57: 112-121.

53 Triant VA. HIV infection and coronary heart disease: an intersection of epidemics. J Infect Dis 2012; 205 (Suppl 3): S355-361.

54 European Medicines Agency. Celsentri: summary of product characteristics. 2015. Available at: http:// www.ema.europa.eu/docs/en_GB/document_library/EPAR__Product_Information/human/000811/WC500022190.pdf (accessed September 2015).

55 Mallon PW. Getting to the heart of HIV and myocardial infarction. JAMA Intern Med 2013; 173: 622-623.

56 Gillard BK, Raya JL, Ruiz-Esponda R et al. Impaired lipoprotein processing in HIV patients on antiretroviral therapy: aberrant high-density lipoprotein lipids, stability, and function. Arterioscler Thromb Vasc Biol 2013; 33: 1714-1721.

57 Keene D, Price C, Shun-Shin MJ, Francis DP. Effect on cardiovascular risk of high density lipoprotein targeted drug treatments niacin, fibrates, and CETP inhibitors: metaanalysis of randomised controlled trials including 117,411 patients. BMJ 2014; 349: g4379.

58 Friis-Moller N, Sabin CA, Weber R et al. Combination antiretroviral therapy and the risk of myocardial infarction. N Engl J Med 2003; 349: 1993-2003.

59 Crum NF, Riffenburgh RH, Wegner S et al. Comparisons of causes of death and mortality rates among HIV-infected persons: analysis of the pre-, early, and late HAART (highly active antiretroviral therapy) eras. J Acquir Immune Defic Syndr 2006; 41: 194-200.

60 Palella FJ Jr, Baker RK, Moorman AC et al. Mortality in the highly active antiretroviral therapy era: changing causes of death and disease in the HIV outpatient study. $J$ Acquir Immune Defic Syndr 2006; 43: 27-34.

61 Bonnet F, Chene G, Thiebaut R et al. Trends and determinants of severe morbidity in HIV-infected patients: the ANRS C03 Aquitaine Cohort, 2000-2004. HIV Med 2007; 8: 547-554.

62 Triant VA, Regan S, Lee $\mathrm{H}$ et al. Association of immunologic and virologic factors with myocardial infarction rates in a US healthcare system. $J$ Acquir Immune Defic Syndr 2010; 55: 615-619.

63 Achhra AC, Amin J, Law MG et al. Immunodeficiency and the risk of serious clinical endpoints in a well studied 
cohort of treated HIV-infected patients. AIDS 2010; 24: 1877-1886.

64 Lichtenstein KA, Armon C, Buchacz K et al. Low CD4 + T cell count is a risk factor for cardiovascular disease events in the HIV outpatient study. Clin Infect Dis 2010; 51: 435447.

65 Zhang S, van Sighem A, Kesselring A et al. Episodes of HIV viremia and the risk of non-AIDS diseases in patients on suppressive antiretroviral therapy. J Acquir Immune Defic Syndr 2012; 60: 265-272.

66 Silverberg MJ, Leyden WA, Xu L et al. Immunodeficiency and risk of myocardial infarction among HIV-positive individuals with access to care. J Acquir Immune Defic Syndr 2014; 65: 160-166.

67 Sax PE, Tierney C, Collier AC et al. Abacavir/lamivudine versus tenofovir DF/emtricitabine as part of combination regimens for initial treatment of HIV: final results. $J$ Infect Dis 2011; 204: 1191-1201.

68 Puls R, Amin J, Losso M et al. Efficacy of $400 \mathrm{mg}$ efavirenz versus standard $600 \mathrm{mg}$ dose in HIV-infected, antiretroviralnaive adults (ENCORE1): a randomised, double-blind, placebo-controlled, non-inferiority trial. Lancet 2014; 383: 1474-1482.

69 van Leth F, Phanuphak P, Stroes E et al. Nevirapine and efavirenz elicit different changes in lipid profiles in antiretroviral-therapy-naive patients infected with HIV-1. PLoS Med 2004; 1: e19.

70 Molina JM, Cahn P, Grinsztejn B et al. Rilpivirine versus efavirenz with tenofovir and emtricitabine in treatmentnaive adults infected with HIV-1 (ECHO): a phase 3 randomised double-blind active-controlled trial. Lancet 2011; 378: 238-246.

71 Fatkenheuer G, Duvivier C, Rieger A et al. Lipid profiles for etravirine versus efavirenz in treatment-naive patients in the randomized, double-blind SENSE trial. J Antimicrob Chemother 2012; 67: 685-690.

72 Daar ES, Tierney C, Fischl MA et al. Atazanavir plus ritonavir or efavirenz as part of a 3-drug regimen for initial treatment of HIV-1. Ann Intern Med 2011; 154: 445-456.

73 Soriano V, Arasteh K, Migrone H et al. Nevirapine versus atazanavir/ritonavir, each combined with tenofovir disoproxil fumarate/emtricitabine, in antiretroviral-naive HIV-1 patients: the ARTEN Trial. Antivir Ther 2011; 16: 339-348.

74 Ofotokun I, Ribaudo H, Na L et al. Darunavir or atazanavir vs raltegravir lipid changes are unlinked to ritonavir exposure: ACTG 5257. Conference on Retroviruses and Opportunistic Infections. March 2014. Boston, MA, USA.

75 Molina JM, Andrade-Villanueva J, Echevarria J et al. Oncedaily atazanavir/ritonavir versus twice-daily lopinavir/ ritonavir, each in combination with tenofovir and emtricitabine, for management of antiretroviral-naive HIV- 1-infected patients: 48 week efficacy and safety results of the CASTLE study. Lancet 2008; 372: 646-655.

76 Raffı F, Rachlis A, Stellbrink H-J et al. Once-daily dolutegravir (DTG; S/GSK1349572) is non-inferior to raltegravir (RAL) in antiretroviral-naive adults: 48 week results from SPRING-2 (ING113086). International AIDS Conference. July 2012. Washinton, DC, USA.

77 Lennox JL, Dejesus E, Berger DS et al. Raltegravir versus efavirenz regimens in treatment-naive HIV-1-infected patients: 96-week efficacy, durability, subgroup, safety, and metabolic analyses. J Acquir Immune Defic Syndr 2010; 55: 39-48.

78 Wohl DA, Cohen C, Gallant JE et al. A randomized, doubleblind comparison of single-tablet regimen elvitegravir/ cobicistat/emtricitabine/tenofovir DF versus single-tablet regimen efavirenz/emtricitabine/tenofovir DF for initial treatment of HIV-1 infection: analysis of week 144 results. J Acquir Immune Defic Syndr 2014; 65: e118-120.

79 Clumeck N, Molina JM, Henry K et al. A randomized, double-blind comparison of single-tablet regimen elvitegravir/cobicistat/emtricitabine/tenofovir DF vs ritonavir-boosted atazanavir plus emtricitabine/tenofovir DF for initial treatment of HIV-1 infection: analysis of week 144 results. J Acquir Immune Defic Syndr 2014; 65: e121124.

80 DeJesus E, Walmsley S, Cohen C et al. Fasted lipid changes after administration of maraviroc or efavirenz in combination with zidovudine and lamivudine (Combivir) for 48 weeks to treatment-naive HIV-infected patients. Conference on Retroviruses and Opportunistic Infections. February 2008. Boston, MA, USA.

81 Campo R, DeJesus E, Bredeek UF et al. SWIFT: prospective 48-week study to evaluate efficacy and safety of switching to emtricitabine/tenofovir from lamivudine/abacavir in virologically suppressed HIV-1 infected patients on a boosted protease inhibitor containing antiretroviral regimen. Clin Infect Dis 2013; 56: 1637-1645.

82 Gupta SK, Mi D, Moe SM et al. Effects of switching from efavirenz to raltegravir on endothelial function, bone mineral metabolism, inflammation, and renal function: a randomized, controlled trial. J Acquir Immune Defic Syndr 2013; 64: 279-283.

83 Pozniak A, Markowitz M, Mills A et al. Switching to coformulated elvitegravir, cobicistat, emtricitabine, and tenofovir versus continuation of non-nucleoside reverse transcriptase inhibitor with emtricitabine and tenofovir in virologically suppressed adults with HIV (STRATEGYNNRTI): 48 week results of a randomised, open-label, phase 3b non-inferiority trial. Lancet Infect Dis 2014; 14: 590599.

84 Parienti JJ, Massari V, Rey D et al. Efavirenz to nevirapine switch in HIV-1-infected patients with dyslipidemia: a 
randomized, controlled study. Clin Infect Dis 2007; 45: 263-266.

85 Mills AM, Cohen C, Dejesus E et al. Efficacy and safety 48 weeks after switching from efavirenz to rilpivirine using emtricitabine/tenofovir disoproxil fumarate-based singletablet regimens. HIV Clin Trials 2013; 14: 216-223.

86 Palella FJ Jr, Fisher M, Tebas P et al. Simplification to rilpivirine/emtricitabine/tenofovir disoproxil fumarate from ritonavir-boosted protease inhibitor antiretroviral therapy in a randomized trial of HIV-1 RNA-suppressed participants. AIDS 2014; 28: 335-344.

87 Waters L, Fisher M, Winston A et al. A phase IV, doubleblind, multicentre, randomized, placebo-controlled, pilot study to assess the feasibility of switching individuals receiving efavirenz with continuing central nervous system adverse events to etravirine. AIDS 2011; 25: 65-71.

88 Young B, Squires KE, Ross LL et al. Inflammatory biomarker changes and their correlation with Framingham cardiovascular risk and lipid changes in antiretroviral-naive HIV-infected patients treated for 144 weeks with abacavir/ lamivudine/atazanavir with or without ritonavir in ARIES. AIDS Res Hum Retroviruses 2013; 29: 350-358.

89 Martinez E, Larrousse M, Llibre JM et al. Substitution of raltegravir for ritonavir-boosted protease inhibitors in HIVinfected patients: the SPIRAL study. AIDS 2010; 24: 16971707.

90 Mallolas J, Podzamczer D, Milinkovic A et al. Efficacy and safety of switching from boosted lopinavir to boosted atazanavir in patients with virological suppression receiving a LPV/r-containing HAART: the ATAZIP study. J Acquir Immune Defic Syndr 2009; 51: 29-36.

91 Eron JJ, Young B, Cooper DA et al. Switch to a raltegravirbased regimen versus continuation of a lopinavir-ritonavirbased regimen in stable HIV-infected patients with suppressed viraemia (SWITCHMRK 1 and 2): two multicentre, double-blind, randomised controlled trials. Lancet 2010; 375: 396-407.

92 Arribas JR, Pialoux G, Gathe J et al. Simplification to coformulated elvitegravir, cobicistat, emtricitabine, and tenofovir versus continuation of ritonavir-boosted protease inhibitor with emtricitabine and tenofovir in adults with virologically suppressed HIV (STRATEGY-PI): 48 week results of a randomised, open-label, phase 3b, non-inferiority trial. Lancet Infect Dis 2014; 14: 581-589.

\subsection{Women}

\subsubsection{Introduction}

The following guidance considers issues concerning the initiation and choice of ART for HIV-positive women who are not currently pregnant. For guidance on the management of pregnancy in HIV-positive woman please refer to the BHIVA guidelines for the management of HIV infection in pregnant women [1].

There are few specific data on ART treatment in women other than in pregnancy. Data available are largely from a meta-analysis, post hoc analyses or derived from cohort studies. The majority of the randomised clinical trial data on ART comes from studies that have enrolled mostly male subjects. If randomised controlled trials do enrol women, the numbers are often too small to draw significant gender-based conclusions.

Approximately one-third of people diagnosed with, and accessing care, for HIV in the UK are women [2]. The majority are of childbearing age but the age range is increasing, adding the complexity of menopause and its sequelae to the management of HIV-positive women. Many HIV-positive women in the UK are of African heritage and face overlapping challenges to their health and well being [3].

Women's experience of HIV reflects multiple social and cultural influences, which when combined with sex-specific biological factors influence individual responses to HIV.

\subsubsection{When to start}

8.7.2.1 Recommendations

- We recommend therapy-naïve HIV-positive women who are not pregnant start ART (see Section 4) (1A).

8.7.2.2 Auditable measure

- Proportion of HIV-positive women not on ART.

8.7.2.3 Rationale

Gender differences in HIV viral load and CD4 cell count at different stages of infection have been observed [4] but have not been consistently associated with long-term clinical outcomes for HIV-positive women. Based on current data, the indications for starting ART do not differ between non-pregnant women and men.

Gender-specific socio-economic and cultural factors may impact on women's ability to access care and manage their medication, compromising their ability to initiate and adhere to therapy, necessitating support from the multidisciplinary team.

\subsubsection{What to start}

8.7.3.1 Recommendations

- There are insufficient data to support specific recommendations for HIV-positive non-pregnant women. We 
therefore recommend therapy-naïve HIV-positive women start ART as per general guidelines (1A).

- We recommend both HIV-positive women of childbearing potential and healthcare professionals who prescribe ART are conversant with the benefits and risks of ARV agents for both the health of the HIV-positive woman and for that of an unborn child (GPP).

- We recommend that potential pharmacokinetic interactions between ARVs, hormonal contraceptive agents and hormone replacement therapy are checked before administration (GPP).

8.7.3.2 Rationale

8.7.3.2.1 Efficacy

There are few data to guide prescribing of initial ART specifically for women as no randomised controlled trial in PLWH starting ART has been powered to detect gender-based differences in efficacy. From the limited data available, virological outcomes within clinical trial settings generally appear to be no different between men and women.

WAVES is a women-only randomised controlled trial that demonstrated superiority of Stribild over Truvada + atazanavir/ritonavir; this was driven predominantly by more adverse event discontinuations in the atazanavir $\operatorname{arm}$ [5].

A meta-analysis of FDA registrational randomised controlled trials analysed data from 20,328 HIV-positive individuals participating in 40 trials for 16 ARVs. Overall, $20 \%$ of study participants were women and there were no clinically or statistically significant differences in week 48 virological outcomes between men and women [6].

A subanalysis of a randomised controlled trial comparing atazanavir/ $\mathrm{r}$ and lopinavir/ $\mathrm{r}$ in ART-naïve individuals of whom 31\% were women, showed comparable virological efficacy at week 96 between the two treatment arms in women [7], although virological response rates were lower in women when compared with men.

In a study comparing atazanavir/r and efavirenz in 1857 ART-naïve individuals of whom 17\% were women, female gender was associated with increased virological failure on atazanavir/r compared with efavirenz [8]. No difference was seen with efavirenz between men and women.

The efficacy and tolerability of raltegravir were similar in men and women at 48 weeks in one cohort of treatment-naïve and -experienced individuals [9]. First-line rilpivirine-based ART showed no difference in rates of virological suppression at 48 and 96 weeks between men and women, but the number of women included was low and the study was not designed to investigate gender differences [10,11].

Cohort studies in the UK have reported similar virological outcomes during the first year of treatment in heterosexual men and women [12]. An Italian cohort study reported no significant effect of gender on clinical progression or the risk of developing a clinical event [13]. Data from Spain, which included both naïve and ARV-experienced women, showed them with similar virological responses to men [14].

HIV-positive women starting ART should use ARVs from the list of preferred and alternative drugs outlined in Section 5.1 .

\subsection{Toxicity, discontinuation and adherence}

Several studies have suggested that gender may influence the frequency, presentation and severity of selected ARTrelated adverse events. Although data are limited, evidence exists that phamacokinetics for some ART drugs may differ between men and women because of factors such as body weight, plasma volume, plasma protein levels, cytochrome P450 activity and drug transporter function $[15,16]$.

Adverse events and treatment discontinuations within ART clinical trials and cohort studies published between 2002 and 2007 have been systematically reviewed. The overall event rate is often the same but the adverse event profile may be different. Women were reported to be more likely than men to experience ART-related lipodystrophy, rash and nausea, and to discontinue therapy [4].

Data from the USA have shown that women are more likely than men to discontinue ART for poor adherence, dermatological symptoms, neurological reasons, constitutional symptoms and concurrent medical conditions [16]. UK cohort data found that $11.4 \%$ of men compared with $19.3 \%$ of women discontinued treatment in the first year of ART (adjusted relative hazard 0.72, 95\% CI 0.63-0.83, $P=0.0001$ ) [12].

An randomised controlled trial of atazanavir/ $\mathrm{r}$ versus lopinavir/r found worse virological outcomes in treatmentnaïve women compared with men due to higher discontinuation rates in women in both treatment arms [7]. CNS side effects of varying severity can occur with efavirenz, particularly at the initiation of therapy. This may be partly explained by the greater efavirenz exposure associated with a CYP2B6 variant, more commonly found in Africans and African Americans [17]. In the UK population, this is of particular relevance to women, the majority of whom are of African ethnicity. Nevirapine-associated rash occurs more frequently in women than men [18]. Hepatotoxicity associated with nevirapine is more common in women with a CD 4 cell count $>250$ cells $/ \mu \mathrm{L}$, or elevated baseline transaminases, and restricts women's use of the drug [19].

Compared with HIV-positive men, women are more likely to experience an increase in central fat with ART [20]. Women have an increased risk of osteopenia/osteoporosis, especially after menopause, and this risk may be exacerbated by HIV and ART [21]. 
At present, these observed differences do not require women-specific recommendations.

A systematic review of studies on gender and ART adherence published between 2000 and 2011 in the resource-rich world concluded that overall reported adherence is lower in women than men [22]. However, of over 1000 studies initially identified for review, only 44 had adequate data on gender to allow any comparisons to be made. The authors identified the particular factors for lower adherence in women were depression, lack of supportive interpersonal relationships, young age, drug and alcohol use, black ethnicity, ART of six or more pills per day, higher numbers of children, self-perception of abdominal fat gain, sleep disturbances and increased levels of distress.

\subsection{Fetal safety}

All women of child-bearing potential should be offered reproductive health counselling including advice around conception, prevention of mother-to-child transmission and contraception as a component of routine medical care [23].

Concerns about potential fetal toxicity of ARVs have influenced prescribing practice in HIV-positive women. Of note, other than zidovudine in the third trimester, no ARV drug has a licence for use in pregnancy.

Pregnancy in women living with HIV who are already on effective therapy is increasing; 80\% of HIV-positive pregnant women in the UK in 2013 were diagnosed before the current pregnancy, of whom 60\% were already on ART at conception [24]. Where newer drugs are available, women are conceiving on these agents, with zidovudine now rarely used as first-line therapy for adults. European cohort data comparing pregnancies that were managed with zidovudine-containing regimens $v s$. those without zidovudine found no difference in risk of detectable viral load at delivery, mother-to-child transmission or congenital abnormality when comparing zidovudinesparing with zidovudine-containing ART [25].

The most robust data on teratogenicity and first trimester ART exposure are from the Antiretroviral Pregnancy Registry (APR) [26]. This international prospective reporting system records rates of congenital birth defects in babies born to women with exposure to ART at any stage of pregnancy. Approximately 200 or more reports need to be received for a particular compound before data are reported for that compound by the APR. There are now over 200 prospective reports in the APR of first trimester exposure for abacavir, atazanavir, darunavir, efavirenz, emtricitabine, lamivudine, lopinavir, nevirapine, ritonavir, tenofovir-DF and zidovudine. No signal of increased risk of congenital abnormality has been demonstrated, and a greater than twofold higher rate than in the general population has been excluded. There are, so far, fewer than
200 prospective reports for raltegravir and rilpivirine within the APR and hence reports on these agents are not yet available.

Despite previous concerns over the safety of efavirenz based on preclinical animal studies and retrospective case reports in human subjects, the current data do not provide evidence of excess teratogenicity above the expected baseline for infants exposed to efavirenz in the first trimester. Sufficient numbers of first-trimester exposures of efavirenz have been monitored to detect at least a twofold increase in risk of overall birth defects within the APR, and no such increases have been detected to date [26].

Data from Côte d'Ivoire found no significant increased risk of unfavourable pregnancy outcome in women with first-trimester exposure to efavirenz compared with nevirapine [27]. A systematic review and meta-analysis of observational cohorts carried out in 2010 [28] and further updated in 2014 [29] reported birth outcomes among women exposed to efavirenz during the first trimester. No increased risk of overall birth defects among the babies of women exposed to efavirenz during the first trimester compared with exposure to other ARV drugs was found. The prevalence of overall birth defects with first-trimester efavirenz exposure was similar to the ranges reported in the general population.

A review of live births to women with HIV in a large unselected UK population between 1990 and 2007 found no increased risk of abnormalities in infants exposed to efavirenz in the first trimester, providing further reassurance that ART in utero does not pose a major risk of fetal anomaly [30]. Mathematical modelling using North American cohort data has demonstrated a theoretical loss of life expectancy in women who delay efavirenz at initiation of ARV [31].

Based on current evidence, efavirenz can be initiated in women of childbearing potential, can be continued in women who conceive on the drug and commenced in pregnancy but the data should be discussed in detail with the individual woman when deciding on her preferred treatment regimen. Given that no ARV drug is licensed for use in pregnancy apart from zidovudine in the third trimester, a discussion regarding the potential unknown long- and short-term effects on an unborn child should be had with any woman of childbearing potential who commences any ARV drug regimen. Further details can be found in the BHIVA pregnancy guidelines [1].

\subsection{Hormone interactions}

Significant pharmacokinetic and pharmacodynamic interactions have been reported between ARV drugs and hormonal agents and these should be taken into consideration when selecting an ART regimen for women using hormonal contraception. We suggest prescribers refer to the 
summary of product characteristics for individual drugs, the University of Liverpool drug interaction website (www.hiv-interactions.org) or specialist pharmacy advice within their unit/network. Importantly, in addition to cases of contraceptive failure in women on efavirenz-based ART using a contraceptive implant [32], there are now pharmacokinetic data to show a significant reduction in levonorgestrel concentrations in women on efavirenz [33].

\subsection{Menopause}

As the average age of the female population living with HIV increases, more women with HIV reach menopause. The menopause raises a number of issues for women with HIV including menopausal symptoms and increased risk of co-morbidities such as cardiovascular disease and osteoporosis. Although data are limited, there is no evidence that menopause has a direct effect on ART efficacy. A subanalysis of responses to ART among a small number of treatment-naïve pre- and post-menopausal women in a US study found no significant differences in the immunological and virological responses between the two groups [34].

\subsubsection{HIV-positive women experiencing virological failure}

There is very little evidence to guide prescribing ART in HIV-positive women experiencing virological failure on ART, with most studies recruiting approximately 10\% of women. One study investigating darunavir/r in ARTexperienced individuals recruited a large proportion of women and was powered to show a difference in virological efficacy between men and women; this showed higher discontinuation rates among women than men, with nausea being cited as a particular problem, but overall there was no difference in virological efficacy [35]. A further study has reported similar efficacy and tolerability of raltegravir in ART-experienced HIV-positive women [9].

In HIV-positive women experiencing virological failure on ART, the same principles of management and recommendations apply as per HIV-positive men experiencing virological failure.

\subsubsection{Psychosocial issues}

Women living with HIV often have additional vulnerability factors (psychological and social) that can impact on access to and engagement with care and also adherence and treatment outcomes. Such factors include HIV-related stigma, low socio-economic status, culturally defined gender roles and high levels of intimate partner violence. There are higher levels of mental health problems, particularly depression and post-traumatic stress disorder, in women living with HIV compared with the general population, which can also adversely affect outcomes. These issues need to be recognised and identified by healthcare professionals and effective interventions offered, in particular psychosocial and peer support

\subsubsection{References}

1 de Ruiter A, Taylor GP, Clayden P et al. British HIV Association guidelines for the management of HIV infection in pregnant women 2012 (2014 interim review). HIV Med 2014; 15 (Suppl 4): 1-77.

2 Public Health England. HIV in the United Kingdom: 2014 Report. London, PHE; 2014. Available at: https:// www.gov.uk/government/uploads/system/uploads/ attachment_data/file/401662/ 2014_PHE_HIV_annual_report_draft_Final_07-01-2015.pdf (accessed June 2015).

3 Doyal L. Challenges in researching life with HIV/AIDS: an intersectional analysis of black African migrants in London. Cult Health Sex 2009; 11: 173-188.

4 Nicastri E, Leone S, Angeletti C et al. Sex issues in HIV-1infected persons during highly active antiretroviral therapy: a systematic review. J Antimicrob Chemother 2007; 60: 724-732.

5 Squires K, Kityo C, Hodder S et al. Elvitegravir (EVG)/cobicistat (COBI)/emtricitabine (FTC)/tenofovir disoproxil fumarate (TDF) is superior to ritonavir (RTV) boosted atazanavir (ATV) plus FTC/TDF in treatment naïve women with HIV-1 infection (WAVES Study). IAS Conference on HIV Pathogenesis, Treatment and Prevention. July 2015. Vancouver, Canada [Abstract MOLBPE08].

6 Soon GG, Min M, Struble KA et al. Meta-analysis of gender differences in efficacy outcomes for HIV-positive subjects in randomized controlled clinical trials of antiretroviral therapy (2000-2008). AIDS Patient Care STDS 2012; 26: 444-453.

7 Squires KE, Johnson M, Yang R et al. Comparative gender analysis of the efficacy and safety of atazanavir/ritonavir and lopinavir/ritonavir at 96 weeks in the CASTLE study. $J$ Antimicrob Chemother 2011; 66: 363-370.

8 Smith KY, Tierney C, Mollan K et al. Outcomes by sex following treatment initiation with atazanavir plus ritonavir or efavirenz with abacavir/lamivudine or tenofovir/ emtricitabine. Clin Infect Dis 2014; 58: 555-563.

9 Squires KE, Bekker LG, Eron JJ et al. Safety, tolerability, and efficacy of raltegravir in a diverse cohort of HIVinfected patients: 48-week results from the REALMRK Study. AIDS Res Hum Retroviruses 2013; 29: 859-870.

10 Cohen CJ, Andrade-Villanueva J, Clotet B et al. Rilpivirine versus efavirenz with two background nucleoside or nucleotide reverse transcriptase inhibitors in treatment-naive adults infected with HIV-1 (THRIVE): a phase 3, randomised, non-inferiority trial. Lancet 2011; 378: 229-237.

11 Martorell C, Mayer CA, Northland R et al. Week 96 safety and efficacy by gender and race subgroups in treatment- 
naïve HIV-1-infected patients in the Phase III ECHO and THRIVE trials. Annual Meeting of the Infectious Diseases Society of America. September 2011. Boston, MA, USA.

12 Barber TJ, Geretti AM, Anderson J et al. Outcomes in the first year after initiation of first-line HAART among heterosexual men and women in the UK CHIC Study. Antivir Ther 2011; 16: 805-814.

13 Murri R, Lepri AC, Phillips AN et al. Access to antiretroviral treatment, incidence of sustained therapy interruptions, and risk of clinical events according to sex: evidence from the I.Co.N.A. Study. J Acquir Immune Defic Syndr 2003; 34: 184-190.

14 Collazos J, Asensi V, Carton JA. Sex differences in the clinical, immunological and virological parameters of HIVinfected patients treated with HAART. AIDS 2007; 21: 835843.

15 Kempf MC, Pisu M, Dumcheva A et al. Gender differences in discontinuation of antiretroviral treatment regimens. $J$ Acquir Immune Defic Syndr 2009; 52: 336-341.

16 Ofotokun I, Chuck SK, Hitti JE. Antiretroviral pharmacokinetic profile: a review of sex differences. Gend Med 2007; 4: 106-119.

17 Haas DW, Ribaudo HJ, Kim RB et al. Pharmacogenetics of efavirenz and central nervous system side effects: an Adult AIDS Clinical Trials Group study. AIDS 2004; 18: 23912400.

18 Mazhude C, Jones S, Murad S et al. Female sex but not ethnicity is a strong predictor of non-nucleoside reverse transcriptase inhibitor-induced rash. AIDS 2002; 16: 1566 1568.

19 Sanne I, Mommeja-Marin H, Hinkle J et al. Severe hepatotoxicity associated with nevirapine use in HIVinfected subjects. J Infect Dis 2005; 191: 825-829.

20 Galli M, Veglia F, Angarano G et al. Gender differences in antiretroviral drug-related adipose tissue alterations. Women are at higher risk than men and develop particular lipodystrophy patterns. J Acquir Immune Defic Syndr 2003; 34: 58-61.

21 Brown TT, Qaqish RB. Response to Berg et al. 'Antiretroviral therapy and the prevalence of osteopenia and osteoporosis: a meta-analytic review'. AIDS 2007; 21: 1830-1831.

22 Puskas CM, Forrest JI, Parashar S et al. Women and vulnerability to HAART non-adherence: a literature review of treatment adherence by gender from 2000 to 2011. Curr HIV/AIDS Rep 2011; 8: 277-287.

23 BHIVA. British HIV Association Standards of Care for People Living with HIV, 2013. Available at: www.bhiva.org/standards-of-care-2013.aspx (accessed June 2015).
24 National Study of HIV in Pregnancy and Childhood. National surveillance data. Available at: www.nshpc.ucl.ac.uk (accessed January 2015).

25 Tariq S, Townsend CL, Cortina-Borja M et al. Use of zidovudine-sparing HAART in pregnant HIV-infected women in Europe: 2000-2009. J Acquir Immune Defic Syndr 2011; 57: 326-333.

26 Antiretroviral Pregnancy Registry Steering Committee. Antiretroviral Pregnancy Registry International Interim Report for 1 January 1989 through 31 July 2014. 2011. Available at: www.apregistry.com/forms/interim_report.pdf (accessed June 2015).

27 Ekouevi DK, Coffie PA, Ouattara E et al. Pregnancy outcomes in women exposed to efavirenz and nevirapine: an appraisal of the IeDEA West Africa and ANRS Databases, Abidjan, Cote d'Ivoire. J Acquir Immune Defic Syndr 2011; 56: 183-187.

28 Ford N, Mofenson L, Kranzer K et al. Safety of efavirenz in first-trimester of pregnancy: a systematic review and metaanalysis of outcomes from observational cohorts. AIDS 2010; 24: 1461-1470.

29 Ford N, Mofenson L, Shubber Z et al. Safety of efavirenz in the first trimester of pregnancy: an updated systematic review and meta-analysis. AIDS 2014; 28 (Suppl 2): S123-131.

30 Townsend CL, Willey BA, Cortina-Borja M et al. Antiretroviral therapy and congenital abnormalities in infants born to HIV-infected women in the UK and Ireland, 1990-2007. AIDS 2009; 23: 519-524.

31 Hsu HE, Rydzak CE, Cotich KL et al. Quantifying the risks and benefits of efavirenz use in HIV-infected women of childbearing age in the USA. HIV Med 2011; 12: 97-108.

32 Leticee N, Viard JP, Yamgnane A et al. Contraceptive failure of etonogestrel implant in patients treated with antiretrovirals including efavirenz. Contraception 2012; 85: 425-427.

33 Scarsi K, Lamorde M, Darin K et al. Efavirenz- but not nevirapine-based antiretroviral therapy decreases exposure to the levonorgestrel released from a sub-dermal contraceptive implant. J Int AIDS Soc 2014; 17: 19484.

34 Patterson KB, Cohn SE, Uyanik J et al. Treatment responses in antiretroviral treatment-naive premenopausal and postmenopausal HIV-1-infected women: an analysis from AIDS Clinical Trials Group Studies. Clin Infect Dis 2009; 49: 473-476.

35 Currier J, Averitt Bridge D, Hagins D et al. Sex-based outcomes of darunavir-ritonavir therapy: a single-group trial. Ann Intern Med 2010; 153: 349-357. 


\subsection{Mental health}

\subsubsection{Recommendations}

- What to start: we recommend that efavirenz-containing regimens be avoided in individuals with a current or past history of depression, psychosis, suicidal ideation or attempted suicide, or at risk of self-harm (1C).

- Switching therapy: we recommend that efavirenz-containing regimens should be switched promptly to a viable alternative when PLWH present with depression, psychosis, suicidal ideation or attempted suicide, or self-harm (1C).

\subsubsection{Rationale}

The summary of product characteristics for efavirenz cautions that "patients with a prior history of psychiatric disorders appear to be at greater risk of serious psychiatric adverse reactions' with a $2 \%$ risk of both severe depression and suicidal ideation [1]. In view of this warning, studies exploring efavirenz and risk of depression or suicide are inevitably subject to confounding by indication because individuals most at risk will not have been prescribed efavirenz or entered into randomised controlled trials where one of the arms included efavirenz.

A meta-analysis of four ACTG randomised controlled trials with efavirenz in one arm included 5000 PLWH [2]. 'Suicidality' was defined as suicidal ideation or attempted or completed suicide. The incidence of suicidality was 8/ 1000 patient years (PY) on efavirenz versus 4/1000 PY without it (hazard ratio [HR] 2.3, $P=0.006$ ). Rates of attempted or completed suicide were 3 versus 1/1000 PY respectively (HR 2.6, $P=0.065$ ) (eight suicides on efavirenz versus one on comparator regimens). In a secondary analysis of time to suicidal ideation, attempted or completed suicide, or death attributed to substance abuse, homicide or accident (to capture possible under-reporting of suicide) rates were 9 and 5/1000 PY respectively (HR 2.06, $P=0.007$ ). Incidence of suicidality did not change over length of follow up indicating that risk could emerge at any time.

A number of studies has examined the CNS effects of switching away from efavirenz but did not detect an effect on mood. However, it should be noted that the event rates reported in the ACTG studies were low $(<1 /$ 100 patient years) and hence the switch studies may have lacked power to detect an effect on mood.

A small Spanish cohort study found no association between depression or suicide attempts and efavirenz but the overall event rate was unusually low and the proportion of those with depression prescribed efavirenz was half that in the main cohort suggesting significant confounding by indication (i.e. less use of efavirenz where there was a concern about mental health) [3]. No association was found in the D:A:D cohort study between efavirenz use and suicide as a reported cause of death, possibly for similar confounding reasons [4].

A retrospective analysis performed by the manufacturer of the US Food and Drug Administration's Adverse Event Reporting System (FAERS) (i.e. post-marketing surveillance data of spontaneous adverse-event reports from PLWH and healthcare workers) explored the ratio of observed to expected number of suicidality events $(0 / E$ ratio) for a variety of drugs [5]. Such data are inevitably subject to reporting biases that make them difficult to interpret. They concluded that there was no association between efavirenz exposure and suicidality because the 0/E ratio did not exceed the arbitrarily predefined threshold of 2, while it did for other drugs with a known suicide risk (e.g. fluoxetine). Nevertheless, the 0/E ratio for efavirenz was significantly higher than for other ARVs, which is consistent with an increased risk for this drug.

Completed suicide must rank among the most adverse possible effects of any treatment. Unfortunately, depression is under-recognised by PLWH and poorly elicited by healthcare workers [6]. The above data support a precautionary stance of avoiding efavirenz in those with a current or past history of depression or suicidality.

\subsubsection{References}

1 European Medicines Agency. Sustiva: summary of product characteristics. 2015. Available at: www.ema.europa.eu/docs/en_GB/document_library/EPAR__Product_Information/human/000249/WC500058311.pdf (accessed September 2015).

2 Mollan KR, Smurzynski M, Eron JJ et al. Association between efavirenz as initial therapy for HIV-1 infection and increased risk for suicidal ideation or attempted or completed suicide: an analysis of trial data. Ann Intern Med 2014; 161: 1-10.

3 Gutierrez F, Garcia L, Padilla S et al. Risk of clinically significant depression in HIV-infected patients: effect of antiretroviral drugs. HIVMed 2014; 15: 213-223.

4 Smith C, Ryom L, d'Arminio Monforte A et al. Lack of association between use of efavirenz and death from suicide: evidence from the D:A:D study. J Int AIDS Soc 2014; 17(Suppl 3): 19512 [Abstract 0315].

5 Napoli AA, Wood JJ, Coumbis JJ et al. No evident association between efavirenz use and suicidality was identified from a disproportionality analysis using the FAERS database. J Int AIDS Soc 2014; 17: 19214.

6 National Institute for Health and Care Excellence. Depression: the treatment and management of depression in adults (updated edition). CG90. The identification of depression in primary care and community settings (pp 98- 
120). 2009. Available at: www.nice.org.uk/guidance/cg90/ evidence/cg90-depression-in-adults-full-guidance2.pdf (accessed September 2015).

\subsection{Adolescents}

Adolescents include all young people defined by WHO as those aged between 10 and 19 years, and young adults aged between 20 and 24 years [1]. For the purposes of these guidelines we will consider adolescents living with HIV by route of transmission: perinatally acquired HIV infection (PaHIV) and behaviourally infected HIV (BaHIV).

For behaviourally infected young people $>18$ years of age, the management of their HIV disease and associated considerations should be in accordance with BHIVA adult guidelines. The management of adolescents aged less than 16 years within paediatric care should be in accordance with Children's HIV Association (CHIVA) guidelines (http://www.chiva.org.uk/professionals/health/guidelines/ index.html) and the Paediatric European Network for Treatment of AIDS (PENTA) treatment guidelines [2]. There are limited data and no randomised controlled trial data on long-term complications of PaHIV and ART exposure throughout physical maturity, and the following recommendations are based on pragmatic and good clinical practice.

8.9.1 Recommendations for management of HIV, ART and sexual and reproductive health specifically for perinatally acquired HIV

- Avoid standard-dose (600 mg) efavirenz-based regimens in any young person $<50 \mathrm{~kg}$, with any history of mental health or psychological or neurocognitive problems.

Young adults and adolescents (YAA) represent a uniquely vulnerable group who have poor health outcomes compared to younger children and older adults living with the same condition. This is a feature of lifestyle, adolescent behaviour, lack of engagement in health care services and primary care and often lack of social support. As such, any service providing care for YAA living with HIV must offer appropriate youth-centred services, with an open-door policy, non-judgemental care provision, and opening hours consistent with educational commitments.

\subsubsection{UK epidemiology for YAA with PaHIV}

With antiretroviral therapy, the significant fall in HIVassociated morbidity and mortality for perinatally infected children has resulted in increasing numbers entering adolescence and transitioning towards adult services [3,4]. Over 90\% of children diagnosed in the UK and reported to the National study of HIV and Pregnancy
(NSHPC) are followed prospectively in the Collaborative HIV Paediatric Study (CHIPS; www.chipscohort.ac.uk). Data to the end of March 2014 shows that of 1873 children ever reported, 595 have already transferred to adult services, at a median age of transfer of 17 years [4].

\subsubsection{Transition process for YAA with PaHIV}

Transfer to adult services had been associated with increased disease-related morbidity and mortality for a wide range of chronic conditions of childhood prompting the National Service Framework (NSF) 2004 to set standards for the healthcare of young people [5]. Subsequently the Department of Health (DH) has produced a wealth of resources to guide the development of transitional care services [6-8]. Transition is defined as 'A planned, purposeful, process resulting in the point of transfer to adult services'. While several different transition models are described, the key to a successful transition is communication, forward planning and maintaining a young person-centred approach $[9,10]$. HIV-specific transitional care guidance is available through CHIVA and set within the CHIVA Standards (www.chiva.org.uk) [10].

\subsubsection{UK epidemiology for YAA with BaHIV}

Public Health England (PHE) surveillance data reveals 736/5,967 (12\%) of new HIV diagnoses in 2013 were in young adults aged 15-24 years. Routes of transmission were: sex between men $(n=462)$; heterosexual contact $(n=152)$; and IVDU $(n=4)$. Both the proportion and number of new HIV diagnoses among MSM aged 1524 years have increased over the past decade, from $8.7 \%$ $(250 / 2,420)$ in 2004 to $16 \%(460 / 2,950)$ in 2013 [11].

\subsubsection{Neurocognitive impact of HIV in YAA}

The neurocognitive impact of living with HIV on the developing adolescent brain is becoming increasingly apparent, with poorer school performance, increased psychiatric diagnoses and particular difficulties in executive functioning for PaHIV YAA [12-14]. Recent data suggest that more than two-thirds of treatment-naïve BaHIV YAA meet criteria for a diagnosis of HIV-associated neurocognitive disorders, with the most common deficits being in memory and fine motor skills [15]. Optimising virological control with further investigation and referral to expert neurology HIV clinics is recommended.

\subsubsection{Antiretroviral therapy}

8.9.6.1 Adherence

Poorer adherence to ART is reported with increasing age in childhood and in behaviourally infected young people when compared to older adults [16-18]. PHE data suggests that those aged 15-24 with a CD4 cell count $<350$ 
cells $/ \mu \mathrm{L}$ are less likely than older adults to be on treatment and for those taking ART, YAA are less likely to achieve viral suppression when compared to older adults [11]. YAA initiating ART therefore require additional adherence support with specific guidance for this population available in the HIV Young Peoples Network Adherence Guidelines (www.hypnet.org.uk). We suggest starting with a robust regimen initially (once daily boosted PI plus two NRTIs) then switching to an NNRTI or integrase single fixed-dose combination for those who achieve virological suppression. PI-based regimens are now the recommended first-line therapy for adolescents in the 2014 PENTA guidelines [2] .

\subsubsection{Toxicity}

At standard dose, increased efavirenz toxicity associated with higher plasma drug levels has been reported in adults of lower weight, a weight band that will include many YAA [19]. Additionally, reports of a potential increase in suicidal risk associated with efavirenz is of concern in an age group where suicide is the second most common cause of death in the UK, and is more than three times as common in males when compared to females [20]. Rates of suicide more than double in those aged 20-24 compared with those aged 15-19; suicide has been reported in PaHIV YAA in adult care [20,21].

Prolonged ART exposure resulting in lipodystrophy, at an age when body image is so important, may have a negative impact on psychological wellbeing and a potential impact on adherence to ART [22,23]. Growth stunting and delayed puberty in PaHIV YAA and dermatological conditions associated with HIV, such as scaring from shingles, molluscum contagiosum and seborrhoeic dermatitis may further exacerbate issues around body image and self worth. Multidisciplinary team assessment that includes dietetics, psychology and where appropriate, referral for cosmetic surgery is required.

8.9.6.3 Resistance

Within the UK paediatric cohort, half of the PaHIV adolescents are triple-class experienced and almost one-tenth of the young people who have ever started ART are off therapy due to poor adherence/refusal, with the risk of onward transmission of resistant virus to partners and offspring $[22,24]$. Decisions as to the optimal regimen for YAA require an individualised approach considering baseline resistance, predicted adherence, substance use and mental health.

\subsubsection{References}

1 World Health Organization. Adolescent health. Available at: www.who.int/topics/adolescent_health/en/ (accessed September 2015).

2 Bamford A, Turkova A, Lyall H et al. Paediatric European Network for Treatment of AIDS (PENTA) guidelines for treatment of paediatric HIV-1 infection 2015: optimizing health in preparation for adult life. HIV Med 2015.

3 Judd A, Doerholt K, Tookey PA et al. Morbidity, mortality, and response to treatment by children in the United Kingdom and Ireland with perinatally acquired HIV infection during 1996-2006: planning for teenage and adult care. Clin Infect Dis 2007; 45: 918-924.

4 Foster C, Judd A, Tookey P et al. Young people in the United Kingdom and Ireland with perinatally acquired HIV: the pediatric legacy for adult services. AIDS Patient Care STDS 2009; 23: 159-166.

5 Department of Health. National service framework: children, young people and maternity services. 2004. Available at: https://www.gov.uk/government/publications/nationalservice-framework-children-young-people-and-maternityservices (accessed September 2015).

6 Department of Health. Transition: getting it right for young people. Best practice guidance. 2006. Available at: http:// webarchive.nationalarchives.gov.uk/20130107105354/http:/ www.dh.gov.uk/en/Publicationsandstatistics/Publications/ PublicationsPolicyAndGuidance/DH_4132145 (accessed September 2015).

7 Department of Health. A transition guide for all services: key information for professionals about the transition process for disabled young people. 2007. Available at: http:// webarchive.nationalarchives.gov.uk/20130401151715/https:/ www.education.gov.uk/publications/standard/ Integratedworking/Page1/DCSF-00776-2007 (accessed September 2015).

8 Foster C. CHIVA guidance on transition for adolescents living with HIV. 2010. Available at: www.chiva.org.uk/files/ 1214/2857/8197/transition.pdf (accessed September 2015).

9 Miles K, Edwards S, Clapson M. Transition from paediatric to adult services: experiences of HIV-positive adolescents. AIDS Care 2004; 16: 305-314.

10 Bundock H, Fidler S, Clarke S et al. Crossing the divide: transition care services for young people with HIV-their views. AIDS Patient Care STDS 2011; 25: 465-473.

11 Yin Z, Brown A, Hughes G et al. HIV in the United Kingdom: 2014 Report. 2014. Available at: https:// www.gov.uk/government/uploads/system/uploads/ attachment_data/file/401662/ 2014_PHE_HIV_annual_report_draft_Final_07-01-2015.pdf (accessed September 2015).

12 Brackis-Cott E, Kang E, Dolezal C et al. Brief report: language ability and school functioning of youth perinatally infected with HIV. J Pediatr Health Care 2009; 23: 158-164.

13 Paramesparan Y, Garvey LJ, Ashby J et al. High rates of asymptomatic neurocognitive impairment in vertically acquired HIV-1-infected adolescents surviving to adulthood. J Acquir Immune Defic Syndr 2010; 55: 134-136. 
14 Wood SM, Shah SS, Steenhoff AP, Rutstein RM. The impact of AIDS diagnoses on long-term neurocognitive and psychiatric outcomes of surviving adolescents with perinatally acquired HIV. AIDS 2009; 23: 1859-1865.

15 Nichols SL, Bethel J, Garvie PA et al. Neurocognitive functioning in antiretroviral therapy-naive youth with behaviorally acquired human immunodeficiency virus. $J$ Adolesc Health 2013; 53: 763-771.

16 Kim SH, Gerver SM, Fidler S, Ward H. Adherence to antiretroviral therapy in adolescents living with HIV: systematic review and meta-analysis. Aids 2014; 28: 19451956.

17 Nachega JB, Hislop M, Nguyen H et al. Antiretroviral therapy adherence, virologic and immunologic outcomes in adolescents compared with adults in southern Africa. $J$ Acquir Immune Defic Syndr 2009; 51: 65-71.

18 Sabin CA, Smith CJ, d'Arminio Monforte A et al. Response to combination antiretroviral therapy: variation by age. AIDS 2008; 22: 1463-1473.

19 Poeta J, Linden R, Antunes MV et al. Plasma concentrations of efavirenz are associated with body weight in HIV-positive individuals. J Antimicrob Chemother 2011; 66: 2601-2604.

20 Mollan KR, Smurzynski M, Eron JJ et al. Association between efavirenz as initial therapy for HIV-1 infection and increased risk for suicidal ideation or attempted or completed suicide: an analysis of trial data. Ann Intern Med 2014; 161: 1-10.

21 Fish R, Judd A, Jungmann E et al. Mortality in perinatally HIV-infected young people in England following transition to adult care: an HIV Young Persons Network (HYPNet) audit. HIV Med 2014; 15: 239-244.

22 European Paediatric Lipodystrophy Group. Antiretroviral therapy, fat redistribution and hyperlipidaemia in HIVinfected children in Europe. AIDS 2004; 18: 1443-1451.

23 Dollfus C, Blanche S, Trocme N et al. Correction of facial lipoatrophy using autologous fat transplants in HIV-infected adolescents. HIV Med 2009; 10: 263-268.

24 Duong T, Judd A, Collins IJ et al. Long-term virological outcome in children on antiretroviral therapy in the UK and Ireland. AIDS 2014; 28: 2395-2405.

\subsection{Bone disease and antiretroviral therapy}

\subsubsection{Introduction}

HIV-1-positive individuals have lower bone mineral density (BMD) at the femoral neck, hip and lumbar spine. In a recent study, HIV was found to be an independent risk factor for low BMD [1]. Among HIV-positive individuals, $25-33 \%$ of those $>40$ years had osteopenia and 5-9\% had osteoporosis [1]. Low BMD in HIV-positive individuals has been associated with traditional risk factors (i.e. older age, female gender, low BMI, smoking and glucocorticoids - including use as part of the treatment of Pneumocystis jirovecii pneumonia) and with HIV-specific factors (i.e. lower nadir CD4 cell count, longer duration of HIV infection and exposure to ART [2]. Similar rates of osteopenia and osteoporosis were observed in a small cohort $(n=33)$ of men with primary HIV-1 infection [3] and men who have sex with men (MSM), irrespective of HIV status or use of amphetamines and other recreational drugs associated with an increased risk of low BMD [4,5]. Several studies have reported an increased rate of fracture of the spine, hip and wrist, with the most pronounced increase in older people living with HIV (>50-60 years) [6-8].

It is important that treatment decisions relative to co-morbidities such as low BMD are placed in context. PLWH may have several co-morbidities and each of these should be considered and weighted appropriately during the treatment-decision process. Furthermore, dual X-ray absorptiometry (DXA) is unable to distinguish low BMD due to osteoporosis from osteomalacia. Vitamin D and parathyroid hormone status should be evaluated and optimised in individuals with low BMD in whom ART or non-ART interventions are being considered.

\subsubsection{When to start antiretroviral therapy}

8.10.2.1 Recommendation

- We recommend that general recommendations for the timing of ART are followed in patients with, or at risk of osteoporosis (1D).

8.10.2.2 Rationale

Initiation of ART is associated with a reduction in BMD of $2-6 \%$; bone loss is generally restricted to the first year with a relatively stable pattern thereafter $[9,10]$. Intermittent use of ART (as compared with continuous, uninterrupted ART) in the SMART study was associated with reduced bone loss during follow up [11]. There are no data to suggest that initiation of ART reduces the rate of bone loss or the risk of fracture [12]; general recommendations for the timing of ART should be followed in HIV populations with, or at risk of, osteoporosis.

\subsubsection{What to start}

8.10.3.1 Recommendations

- We recommend against the use of tenofovir-DF disoproxil fumarate in individuals aged $>40$ years with osteoporosis, a history of fragility fracture, or a FRAX score of $>20 \%$ (major osteoporotic fracture) if acceptable alternative ARV agents are available (1B).

8.10.3.2 Rationale

In clinical trials, ART-naïve individuals who initiated tenofovir-DF-containing regimens experienced greater reductions in BMD than those initiating tenofovir-DFsparing regimens. For example, in the Gilead 903 study, 
observed reductions in lumbar spine and hip BMD at 144 weeks were $2.2 \%$ and $2.8 \%$ in the tenofovir-DF arm and $1.0 \%$ and $2.4 \%$ in the stavudine arm (each co-administered with lamivudine and efavirenz) [13]. In the ASSERT study, the reduction in BMD at the lumbar spine and hip at 48 weeks was $2.4 \%$ and $3.6 \%$ in the tenofovirDF/emtricitabine arm and $1.6 \%$ and $1.9 \%$ in the abacavir/ lamivudine arm (each given with efavirenz) [14], while in the ACTG5224s study, in which the third agent consisted of efavirenz or ritonavir-boosted atazanavir, the observed reductions at 96 weeks were 3.3\% and 4.0\% with tenofovir-DF/emtricitabine and $1.3 \%$ and $2.6 \%$ with abacavir/ lamivudine [15]. When analysed together with two other ACTG studies, randomisation to tenofovir-DF was associated with a 1.4\% greater reduction in total BMD [12]. Considerably smaller BMD reductions (0.6-1\%) were observed following the initiation of tenofovir alafenamide (TAF), a new formulation currently undergoing Phase 3 clinical trial, as compared with tenofovir disoproxil fumarate (2.4-3.4\%) when co-administered with emtricitabine, cobicistat and elvitegravir [16].

Several studies have examined the effects on BMD of PIs as part of initial ART. Greater bone loss at the lumbar spine (3.1 vs. 1.7\%) but not at the hip (3.4\% vs 3.1\%) was observed with atazanavir/ritonavir as compared with efavirenz in ACTG 5224s [15]. When analysed together with two other ACTG studies, randomisation to ritonavirboosted protease inhibitors resulted in a $0.8 \%$ greater reduction in total BMD [12]. A Danish study observed no differences in BMD change between those who were randomised to zidovudine/lamivudine or lopinavir/r, each given with efavirenz. At 144 weeks, lumbar spine BMD had decreased by $1.9 \%$ and $2.5 \%$, respectively, and hip BMD by $5.0 \%$ and $6.1 \%$ [17]. Several recent trials in treatment-naïve subjects showed more favourable BMD changes with raltegravir plus lopinavir/r or darunavir/r as compared with tenofovir-DF/emtricitabine [18-20]. There are currently insufficient data to make recommendations regarding the third agent in terms of their effect on BMD.

\subsubsection{Switching treatment}

\subsubsection{Recommendations}

- We recommend against continued use of tenofovir-DF in individuals >40 years who are diagnosed with osteoporosis, have sustained a fragility fracture, or have a FRAX score of $>20 \%$ (major osteoporotic fracture) if acceptable alternative ARV agents are available (1C).

8.10.4.2 Rationale

Tenofovir-DF and PIs have been associated with low BMD and bone loss in cohort studies [21-25], and use of
tenofovir-DF and lopinavir/r or any PI with an increased incidence of fractures [26,27]. In individuals who were virologically suppressed on ART, a switch from zidovudine/lamivudine to tenofovir-DF/emtricitabine resulted in a $2.1 \%$ reduction in $\mathrm{BMD}$ at the lumbar spine and hip at 48 weeks, compared with no change $(+0.6 \%$ and $+0.3 \%)$ following a switch to abacavir/lamivudine [28]. Similar, albeit statistically not significant reductions in lumbar spine and hip BMD were observed in the PREPARE study in which PLWH were randomised to switch from zidovudine/lamivudine to tenofovir-DF/emtricitabine or remain on their zidovudine/lamivudine-containing regimen [29]. Smaller reductions $(\sim 1.9 \%)$ in lumbar spine and total hip BMD have been observed with raltegravir vs. 2-3 NRTI (each administered with lopinavir/ritonavir) as second line ART [30], and improvements of 2.5-3\% following a switch from tenofovir-DF to raltegravir [31] or a switch from tenofovir-DF/emtricitabine/efavirenz to darunavir/r [32]. There are no data from ART switch studies to suggest that PI discontinuation improves BMD.

of the non-nucleoside reverse transcriptase inhibitors, efavirenz has been associated with vitamin D deficiency in cohort studies and clinical trials [9]. There are no data to suggest that the initiation of efavirenz containing ART results in greater initial bone loss, or that efavirenzcontaining ART is associated with the development of osteoporosis or fractures. A single case of osteomalacia has been reported with the use of efavirenz [33]. Use of PI has been associated with avascular necrosis of bone and these agents may thus be best avoided in those who have developed this complication [34].

\subsubsection{References}

1 Cotter AG, Sabin CA, Simelane S et al. Relative contribution of HIV infection, demographics and body mass index to bone mineral density. AIDS 2014; 28: 2051-2060.

2 McComsey GA, Tebas P, Shane E et al. Bone disease in HIV infection: a practical review and recommendations for HIV care providers. Clin Infect Dis 2010; 51: 937-946.

3 Grijsen ML, Vrouenraets SM, Steingrover R et al. High prevalence of reduced bone mineral density in primary HIV1-infected men. AIDS 2010; 24: 2233-2238.

4 Grijsen ML, Vrouenraets SM, Wit FW et al. Low bone mineral density, regardless of HIV status, in men who have sex with men. $J$ Infect Dis 2013; 207: 386-391.

5 Liu AY, Vittinghoff E, Sellmeyer DE et al. Bone mineral density in HIV-negative men participating in a tenofovir pre-exposure prophylaxis randomized clinical trial in San Francisco. PloS One 2011; 6: e23688.

6 Triant VA, Brown TT, Lee H, Grinspoon SK. Fracture prevalence among human immunodeficiency virus (HIV)infected versus non-HIV-infected patients in a large U.S. 
healthcare system. J Clin Endocrinol Metab 2008; 93: 34993504.

7 Guerri-Fernandez R, Vestergaard P, Carbonell C et al. HIV infection is strongly associated with hip fracture risk, independently of age, gender, and comorbidities: a population-based cohort study. J Bone Miner Res 2013; 28: 1259-1263.

8 Hansen AB, Gerstoft J, Kronborg G et al. Incidence of low and high-energy fractures in persons with and without HIV infection: a Danish population-based cohort study. AIDS 2012; 26: 285-293.

9 Childs K, Welz T, Samarawickrama A, Post FA. Effects of vitamin D deficiency and combination antiretroviral therapy on bone in HIV-positive patients. AIDS 2012; 26: 253-262.

10 Bolland MJ, Grey A, Horne AM et al. Stable bone mineral density over 6 years in HIV-infected men treated with highly active antiretroviral therapy (HAART). Clin Endocrinol 2012; 76: 64-648.

11 Grund B, Peng G, Gibert CL et al. Continuous antiretroviral therapy decreases bone mineral density. AIDS 2009; 23: 1519-1529.

12 Grant PM, Kitch D, McComsey GA et al. Low baseline $\mathrm{CD} 4+$ count is associated with greater bone mineral density loss after antiretroviral therapy initiation. Clin Infect Dis 2013; 57: 1483-1488.

13 Gallant JE, Staszewski S, Pozniak AL et al. Efficacy and safety of tenofovir DF vs stavudine in combination therapy in antiretroviral-naive patients: a 3-year randomized trial. JAMA 2004; 292: 191-201.

14 Stellbrink HJ, Orkin C, Arribas JR et al. Comparison of changes in bone density and turnover with abacavirlamivudine versus tenofovir-emtricitabine in HIV-infected adults: 48-week results from the ASSERT study. Clin Infect Dis 2010; 51: 963-972.

15 McComsey GA, Kitch D, Daar ES et al. Bone mineral density and fractures in antiretroviral-naive persons randomized to receive abacavir-lamivudine or tenofovir disoproxil fumarate-emtricitabine along with efavirenz or atazanavirritonavir: aids clinical trials group A5224s, a substudy of ACTG A5202. J Infect Dis 2011; 203: 1791-1801.

16 Sax PE, Zolopa A, Brar I et al. Tenofovir alafenamide vs. tenofovir disoproxil fumarate in single tablet regimens for initial HIV-1 therapy: a randomized phase 2 study. $J$ Acquir Immune Defic Syndr 2014; 67: 52-58.

17 Hansen $\mathrm{AB}$, Obel N, Nielsen $\mathrm{H}$ et al. Bone mineral density changes in protease inhibitor-sparing vs. nucleoside reverse transcriptase inhibitor-sparing highly active antiretroviral therapy: data from a randomized trial. HIV Med 2011; 12: 157-165.

18 Reynes J, Trinh R, Pulido F et al. Lopinavir/ritonavir combined with raltegravir or tenofovir/emtricitabine in antiretroviral-naive subjects: 96-week results of the
PROGRESS study. AIDS Res Hum Retroviruses 2013; 29: 256-265.

19 Bedimo RJ, Drechsler H, Jain M et al. The RADAR study: week 48 safety and efficacy of RAltegravir combined with boosted DARunavir compared to Tenofovir/Emtricitabine combined with boosted Darunavir in antiretroviral-naive patients. Impact on bone health. PloS One 2014; 9: e106221.

20 Bernardino JI, Mocroft A, Mallon PW et al. Changes in bone mineral density (BMD) over 96 weeks on darunavir/ ritonavir (DRV/r) + raltegravir (RAL) or darunavir/ritonavir + tenofovir/emtricitabine (TDF/FTC). 54th ICAAC. September 2014. Washington, DC, USA [Abstract H-1198].

21 Jacobson DL, Spiegelman D, Knox TK, Wilson IB. Evolution and predictors of change in total bone mineral density over time in HIV-infected men and women in the nutrition for healthy living study. J Acquir Immune Defic Syndr 2008; 49: 298-308.

22 Bonjoch A, Figueras M, Estany C et al. High prevalence of and progression to low bone mineral density in HIVinfected patients: a longitudinal cohort study. AIDS 2010; 24: 2827-2833.

23 Mary-Krause M, Viard JP, Ename-Mkoumazok B et al. Prevalence of low bone mineral density in men and women infected with human immunodeficiency virus 1 and a proposal for screening strategy. J Clin Densitom 2012; 15: 422-433.

24 Yin MT, Zhang CA, McMahon DJ et al. Higher rates of bone loss in postmenopausal HIV-infected women: a longitudinal study. J Clin Endocrinol Metab 2012; 97: 554-562.

25 Kinai E, Nishijima T, Mizushima D et al. Long-term use of protease inhibitors is associated with bone mineral density loss. AIDS Res Hum Retrovirus 2014; 30: 553-559.

26 Bedimo R, Maalouf NM, Zhang S et al. Osteoporotic fracture risk associated with cumulative exposure to tenofovir and other antiretroviral agents. AIDS 2012; 26: 825-831.

27 Womack JA, Goulet JL, Gibert C et al. Increased risk of fragility fractures among HIV infected compared to uninfected male veterans. PloS One 2011; 6: e17217.

28 Rasmussen TA, Jensen D, Tolstrup M et al. Comparison of bone and renal effects in HIV-infected adults switching to abacavir or tenofovir based therapy in a randomized trial. PloS One 2012; 7: e32445.

29 Cotter AG, Vrouenraets SM, Brady JJ et al. Impact of switching from zidovudine to tenofovir disoproxil fumarate on bone mineral density and markers of bone metabolism in virologically suppressed HIV-1 infected patients; a substudy of the PREPARE study. $J$ Clin Endocrinol Metab 2013; 98: 1659-1666.

30 Haskelberg H, Mallon PW, Hoy J et al. Bone mineral density over 96 weeks in adults failing first-line therapy 
randomized to raltegravir/lopinavir/ritonavir compared with standard second-line therapy. J Acquir Immune Defic Syndr 2014; 67: 161-168.

31 Bloch M, Tong WW, Hoy J et al. Switch from tenofovir to raltegravir increases low bone mineral density and decreases markers of bone turnover over 48 weeks. HIV Med 2014; 15: $373-380$.

32 Hamzah L, Tiraboschi JM, Toby M et al. Favourable effect on vitamin D and bone after switching from Atripla to darunavir/ritonavir: a randomisedcontrolled clinical trial. 8th IAS Conference on HIV Pathogenesis, Treatment \& Prevention. July 2015. Vancouver, Canada [Abstract TUPDB0102].

33 Herzmann C, Arasteh K. Efavirenz-induced osteomalacia. AIDS 2009; 23: 274-275.

34 Permpalung N, Ungprasert P, Summachiwakij S et al. Protease inhibitors and avascular necrosis: a systematic review and meta-analysis. Int J Antimicrob Agent 2014; 44: 93-95.

\subsection{Considerations for later life}

\subsubsection{Introduction}

PLWH are not only living into older age but older people are also acquiring HIV as they maintain sexually active lifestyles. The proportion of PLWH in the UK aged 50 and over has more than doubled in the last decade: in 2013, $27 \%(21,910 / 81,512)$ were aged 50 years or above compared with $13 \%(5,286 / 41,153)$ in 2004 [1]. Thirty percent $(10,730 / 25,380)$ of MSM seen for HIV care were aged 50 years or above in 2013 compared to one in five (21\%, $5,390 / 36,230)$ in 2008 [1]. Older PLWH are more likely to experience co-morbidities and be receiving non-ARV medication. In addition, increased age may be associated with mental health issues, social isolation and financial challenges; HIV-treating clinicians should be mindful of these factors and familiar with appropriate sources of support.

\subsubsection{When to start ART}

8.11.2.1 Recommendation

- We recommend standard criteria are used to determine when to commence antiretroviral therapy in older PLWH (1C).

\subsubsection{Rationale}

The following factors should be specifically considered.

\subsection{Rate of CD4 cell count decline}

Older age has been found to be strongly associated with faster CD4 cell count declines [2-4]. A more recent analysis from the COHERE dataset demonstrated older age was significantly associated with higher viral load, which is in turn associated with CD4 cell count decline [5]. As such, older individuals with a high CD4 cell count may experience more rapid decline so older age may be considered an additional factor when deciding how quickly to commence ART at high CD4 strata.

8.11.2.2.2 Absolute risk of disease progression at a given CD4 cell count

The absolute risk of disease progression is significantly higher for a given CD4 cell count in older people, a factor to consider when counselling older individuals about starting ART.

8.11.2.2.3 CD4 cell recovery on commencing antiretroviral therapy

CD4 cell recovery on commencing ART may be limited in the older person [5,6], possibly due to age-associated effects on thymic function or lower baseline CD4 cell counts $[5,7,8]$. Some studies suggest this is a short-term phenomenon attenuated with longer duration of ART [9] and others suggest that CD4 cell recovery and virological suppression are not affected by age $[10,11]$.

8.11.2.2.4 The presence of non-infectious co-morbidities

HIV infection itself may accelerate age-related conditions. PLWH may therefore develop non-infectious co-morbidities at an earlier age; 'accelerated frailty' may be associated with a lower CD4 count and high viral load. While increased frailty has been observed in ART-naïve individuals, and ART may limit this accelerated ageing, long-term ART exposure may also contribute to certain phenotypes associated with co-morbidities, including fat changes, atherosclerosis and sarcopenia [12].

\subsubsection{References}

1 Yin Z, Brown A, Hughes G et al. HIV in the United Kingdom: 2014 Report. 2014. Available at: https:// www.gov.uk/government/uploads/system/uploads/ attachment_data/file/401662/

2014_PHE_HIV_annual_report_draft_Final_07-01-2015.pdf (accessed September 2015).

2 Langford SE, Ananworanich J, Cooper DA. Predictors of disease progression in HIV infection: a review. AIDS Res Ther 2007; 4: 11.

3 Touloumi G, Hatzakis A, Rosenberg PS et al. Effects of age at seroconversion and baseline HIV RNA level on the loss of CD4 + cells among persons with hemophilia. Multicenter Hemophilia Cohort Study. AIDS 1998; 12: 1691-1697.

4 Natural History Project Working Group for the Collaboration of Observational HIV Epidemiological Research Europe in EuroCoord. Factors associated with short-term changes in HIV viral load and CD4(+) cell count in antiretroviral-naive individuals. AIDS 2014; 28: 1351-1356.

5 Grabar S, Kousignian I, Sobel A et al. Immunologic and clinical responses to highly active antiretroviral therapy 
over 50 years of age. Results from the French Hospital Database on HIV. AIDS 2004; 18: 2029-2038.

6 Manfredi R, Chiodo F. A case-control study of virological and immunological effects of highly active antiretroviral therapy in HIV-infected patients with advanced age. AIDS 2000; 14: 1475-1477.

7 Douek DC, McFarland RD, Keiser PH et al. Changes in thymic function with age and during the treatment of HIV infection. Nature 1998; 396: 690-695.

8 Nogueras M, Navarro G, Anton E et al. Epidemiological and clinical features, response to HAART, and survival in HIVinfected patients diagnosed at the age of 50 or more. $B M C$ Infect Dis 2006; 6: 159.

9 Silverberg MJ, Leyden W, Horberg MA et al. Older age and the response to and tolerability of antiretroviral therapy. Arch Intern Med 2007; 167: 684-691.

10 Tumbarello M, Rabagliati R, de Gaetano Donati K et al. Older age does not influence CD4 cell recovery in HIV-1 infected patients receiving highly active antiretroviral therapy. BMC Infect Dis 2004; 4: 46.

11 Wellons MF, Sanders L, Edwards LJ et al. HIV infection: treatment outcomes in older and younger adults. $\mathrm{J} \mathrm{Am}$ Geriatr Soc 2002; 50: 603-607.

12 Guaraldi G, Prakash M, Moecklinghoff C, Stellbrink HJ. Morbidity in older HIV-infected patients: impact of longterm antiretroviral use. AIDS Rev 2014; 16: 75-89.

\subsubsection{What to start}

8.11.3.1 Recommendation

- We recommend standard antiretroviral regimens are commenced in older PLWH (1C).

8.11.3.2 Rationale

The factors below should be specifically considered when commencing therapy in older PLWH.

8.11.3.2.1 Non-infectious comorbidities

Non-infectious comorbidities are more prevalent in older individuals and are reported to occur more frequently and at a younger age in PLWH compared to matched control populations [1]. Considerations regarding the presence of end-organ disease should be taken into account when tailoring antiretroviral therapy for older individuals.

\subsection{Concomitant medication}

The use of concomitant medication, both over-the-counter preparations and prescription medication is highly prevalent in older PLWH [2]. Consideration regarding drug-drug interactions with concomitant medications is required when commencing antiretroviral therapy in older PLWH.

\subsection{Clinical pharmacology and ageing}

All aspects of drug pharmacology, namely absorption, metabolism, distribution and elimination are reported to change with age. Specifically, for the currently available antiretroviral drugs, effects on hepatic metabolism and elimination may be relevant [3]. Regarding hepatic metabolism, CYP3A4 activity may wane with age and therefore, for drugs metabolised via this pathway, plasma exposure may increase with age. In pharmacokinetic studies, exposure of the boosted-protease inhibitors has been reported to increase with age [4], with these effects not reported with other classes such as the integrase inhibitors [5]. Although theoretically this could lead to increased toxicity in older PLWH, this has not been reported in clinical practice. Regarding elimination, renal elimination of drugs reduces with increasing age. Pharmacokinetic studies have described increased exposure of tenofovir-DF in older PLWH compared to younger PLWH, which was thought to be due to reduced renal clearance [6]. Again, here there is a theoretical risk of increased toxicity from higher drug exposure; however, clinical data are lacking.

\subsubsection{References}

1 Schouten J, Wit FW, Stolte IG et al. Cross-sectional comparison of the prevalence of age-associated comorbidities and their risk factors between HIV-infected and uninfected individuals: the AGEhIV cohort study. Clin Infect Dis 2014; 59: 1787-1797.

2 Marzolini C, Back D, Weber R et al. Ageing with HIV: medication use and risk for potential drug-drug interactions. J Antimicrob Chemother 2011; 66: 2107-2111.

3 Winston A, Underwood J. Emerging concepts on the use of antiretroviral therapy in older adults living with HIV infection. Curr Opin Infect Dis 2015; 28: 17-22.

4 Winston A, Jose S, Gibbons S et al. Effects of age on antiretroviral plasma drug concentration in HIV-infected subjects undergoing routine therapeutic drug monitoring. J Antimicrob Chemother 2013; 68: 1354-1359.

5 Vera JH, Jackson A, Dickinson L et al. The pharmacokinetic profile of raltegravir-containing antiretroviral therapy in HIV-infected individuals over 60 years of age. HIV Clin Trials 2015; 16: 39-42.

6 Baxi SM, Greenblatt RM, Bacchetti P et al. Common clinical conditions - age, low BMI, ritonavir use, mild renal impairment - affect tenofovir pharmacokinetics in a large cohort of HIV-infected women. AIDS 2014; 28: 59-66. 


\subsection{Acknowledgements}

The Writing Group thanks Professors Jose Arribas, Francois Raffi and Linos Vandekerckhove for their peer review of the guidelines.

The Writing Group thanks Alison Richards for conducting the systematic literature search; Jacoby Patterson for work on critical appraisal, evidence profiles and construction of GRADE tables; Sara Gibbons and Kay Seden for their work on Appendix 4 Food considerations for antiretrovirals; and the BHIVA Secretariat for administrative help. 


\subsection{List of appendices}

The appendices can be found on the BHIVA website (www.bhiva.org/guidelines.aspx)

Appendix 1. Summary of the modified GRADE system Appendix 2. PICO questions and search strategies
Appendix 3. Grade tables

Appendix 4. Food considerations for antiretrovirals

Appendix 5. Considerations for antiretrovirals in renal impairment 